\title{
Elektroresistiver Effekt im sauerstoffdefizitären Manganit $\mathrm{Nd}_{2 / 3} \mathrm{Sr}_{1 / 3} \mathrm{MnO}_{3-\delta}$
}

\author{
Dissertation \\ zur Erlangung des Doktorgrades \\ der Mathematisch-Naturwissenschaftlischen Fakultäten \\ der Georg-August-Universität zu Göttingen
}

vorgelegt von

Wladimir Morsakov

aus Wedenka bei Kustanai / Kasachstan

Göttingen, 2006 
D7

Referent: Prof. Dr. K. Bärner

Korreferent: Prof. Dr. K. Samwer

Tag der mündlichen Prüfung: 11.07.2006 


\section{Danksagung}

Herrn Prof. Dr. K. Bärner danke ich für die Ermöglichung und die Förderung dieser Arbeit, für hilfreiche und unermüdliche Diskussionsbereitschaft und stetes Interesse am Fortgang dieser Arbeit. Die von ihm sehr positiv geprägte angenehme Arbeitsatmosphäre machte auch das Leben in der Arbeitsgruppe weit über fachliche Themen hinaus möglich. Ihm möchte ich besonders danken.

Herrn Prof. I. Troyanchuk und seine Arbeitsgruppe in Minsk danke ich für die Herstellung der polykristallinen Proben, die ein so breites Spektrum von interessanten Eigenschaften aufweisen.

Das Team des CRISMAT-Labors am ISMRA-Institut (Caen, Frankreich) für die Hilfe bei der Herstellung und Charakterisierung der Schichtproben. Mein Dank besonderer gilt Prof. B. Raveau und Dr. Mercey.

Dr. Axel Kattwinkel möchte ich danken für seine Unterstützung bei der Überwindung der vielen kleinen und großen experimentellen Hürden während meiner ersten Monate in Göttingen.

Der gesamten Arbeitsgruppe von Prof. K. Bärner: Mostafa Boshta, Nagat Hamad, Wolfgang Hahn, Doaa Abdelbarey danke ich für das angenehmen und freundliche Arbeitsklima und für ihre ständige Hilfsbereitschaft.

Den zahlreichen Gäste, die während meiner Zeit bei Prof. K. Bärner zu Gast waren, möchte ich für ihre experimentelle Unterstützung sowie für ihre ständige Diskussionsbereitschaft danken. Insbesondere danke ich Irina Medvedeva aus Russland, Murad Annaorazov aus Turkmenistan, Igor Troyanchuk aus Weisrussland, Chang Ping Yang aus China und Nikolai Tankovsky aus Bulgarien.

Mein herzlicher Dank gilt Margot Gök, die zu der angenehmen Arbeitsatmosphäre in unserer Arbeitsgruppe sehr viel beigetragen hat. Auch danke ich für ihr Interesse an der Verbesserung meiner sprachlichen Kenntnisse und für die semantische Korrektur dieser Arbeit.

Diese Arbeit wurde von dem Deutschen Akademischen Austauschdienst (DAAD), von der CETA-Gesellschaft und von der Otto-Vahlbruch-Stiftung gefördert.

An dieser Stelle möchte ich auch allen danken, die in den letzten Jahren auf vielfältige Weise zum Gelingen dieser Arbeit beigetragen haben. Dabei möchte ich insbesondere die elektronische (Klaus Langohr und Rasit Kösker) und die mechanische (Thomas Lehmann und Andreas Juretzko) Werkstatt des 4. Physikalischen Instituts und die Zentralwerkstatt (Uwe Frenzel und Christof Schmidt) danken. 



\section{Inhaltsverzeichnis}

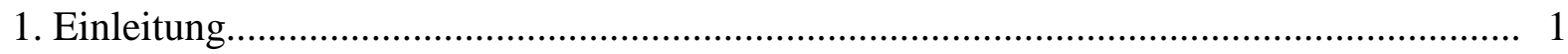

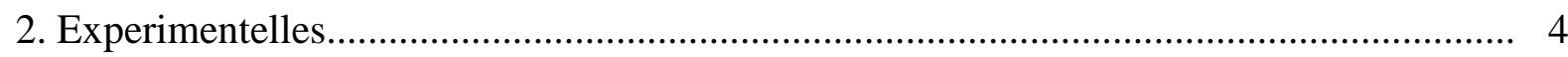

2.1 Präparation der keramischen $\mathrm{Nd}_{2 / 3} \mathrm{Sr}_{1 / 3} \mathrm{MnO}_{3-\delta}$-Proben................................... 4

2.2 Probencharakterisierung.............................................................................. 4

- Röntgenbeugung.......................................................................... 4

- Rasterelektronenmikroskopie (SEM) ............................................ 8

- Transmissionselektronenmikroskopie (TEM)...................................... 9

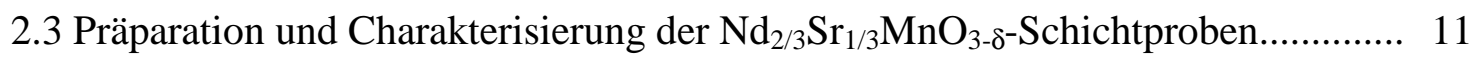

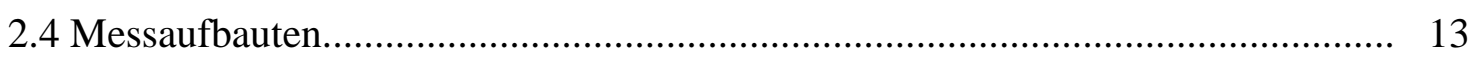

- Die Faraday-Methode zur Messung der Magnetisierung......................... 13

- Spezifische Wärmekapazität............................................................. 14

- Spezifischer Widerstand................................................................... 14

- Bestimmung der Wärmeleitfähigkeit und der Thermokraft durch die Methode der zeitaufgelösten thermoelektrischen Effekte (TTE)........ 15

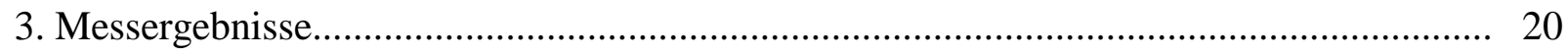

3.1. Temperatur- und Magnetfeldabhängigkeit der Magnetisierung........................ 20

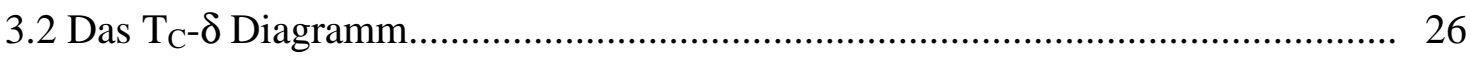

3.3 Spezifischer Widerstand und V(I)-Charakteristiken der keramischen

$\mathrm{Nd}_{2 / 3} \mathrm{Sr}_{1 / 3} \mathrm{MnO}_{3-\delta}$-Proben.......................................................................... 27

3.4 Elektrowiderstand der $\mathrm{Nd}_{2 / 3} \mathrm{Sr}_{1 / 3} \mathrm{MnO}_{3-\delta}$-Schichtproben................................ 35

3.5 Magnetowiderstand der keramischen $\mathrm{Nd}_{2 / 3} \mathrm{Sr}_{1 / 3} \mathrm{MnO}_{3-\delta}$-Proben........................ 39

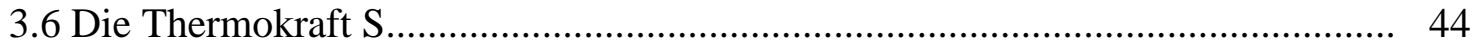

3.7 Die Wärmeleitfähigkeit K............................................................................ 44

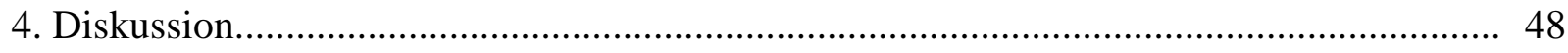

4.1. Physik der Manganite - die Grundideen..................................................... 48

- Elektronische Struktur in einem Kristallfeld und Jahn-Teller-Effekt....... 48

- „Doppelaustauch“-Mechanismus (DA) und Polaronenmodell................. 50

- Goodenough's Bedingung für die ,Zener bond“-Blockierung................. 52

- Phasenseparation oder kohärent gekantete Phasen?............................... 52

- $\quad$ Superaustausch (SA).................................................................... 53

- Kohärent gekanteter Zustand............................................................. 53

- Lokaler Doppelaustausch und lokale Spinkantung.................................. 55

- Phasenseparation.............................................................................. 56

- Pm- und fm-Cluster.................................................................... 58

- Blockierung der Phasenseparation......................................................... 59 
4.2. Kopplung der Untersysteme in Manganiten................................................ 60

4.3. Die auf die Manganite wirkenden thermodynamischen Kräfte......................... 61

- Entropische Kraft........................................................................... 61

- Druckinduzierte Kraft..................................................................... 61

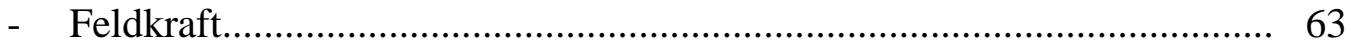

- Chemisches Potential........................................................................ 64

- Elektrisches Feld und elektrische Dipole in Manganiten.......................... 66

4.4. Elektronische Zustände in sauerstoffdefizitären Manganiten............................. 67

- Elektronische Zustände in DA-SA Systemen......................................... 68

- Erweitertes deGennes Diagramm für die Halbmetallsituation.................. 70

- Verschiebung der $\mathrm{T}_{1}$ und $\mathrm{T}_{\mathrm{mi}}$ Temperaturen im H-Feld......................... 71

- Durch das E-Feld induzierte Ordnungs-Ordnungs Phasenübergänge....... 73

- Intrinsischer elektroresistiver Effekt......................................................... 75

- Spinabhängiger Barrierenwiderstand................................................... 77

5. Zusammenfassung und Ausblick.................................................................... 79

Anhang A: Widerstandsinstabilitäten........................................................... 81

Anhang B: Doppelwechselfeldmethode zur Bestimmung des barischen

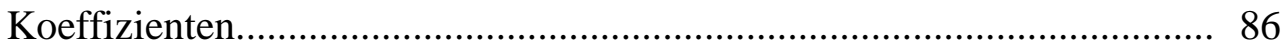

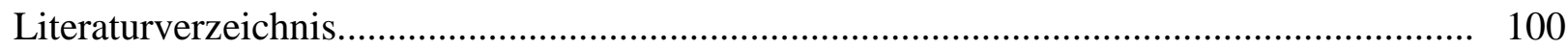




\section{Einleitung.}

Die Perowskite $^{1}$ zeigen ein überraschend breites Spektrum von physikalischen Eigenschaften. Das sind z.B.: Ferromagnetismus, Ferroelektrizität, Hochtemperatur-Supraleitung u.s.f.. Im Jahr 1950 wurde über die Korrelation zwischen Ferromagnetismus und metallischer Leitfähigkeit in den dotierten Manganiten der seltenen Erden (R) berichtet, $R_{1-x} D_{x} M_{n} O_{3}$ (D = Erdalkali) [114, 115]. Vier Jahre später wurde die erste Messung des übergroßen negativen Magnetowiderstandes (CMR) an einer keramischen $\mathrm{La}_{0.8} \mathrm{Sr}_{0.2} \mathrm{MnO}_{3}$-Probe veröffentlicht (Abb. 1.1) [116].

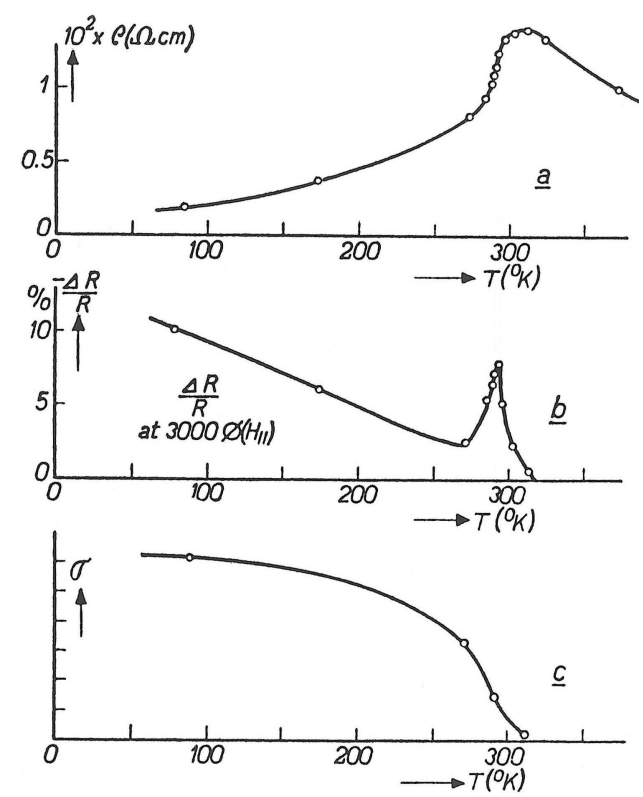

Abbildung 1.1: Messung des negativen magnetoresistiven Effektes an einer keramischen $\mathrm{La}_{0.8} \mathrm{Sr}_{0.2} \mathrm{MnO}_{3}$-Probe und Korrelation zwischen Ferromagnetismus und metallischer Leitfähigkeit, nach [116, veröffentlicht im Jahr 1954].

Bei neueren Untersuchungen des negativen CMR-Effektes, $M R=\Delta \rho / \rho(0)=[\rho(H)-\rho(0)] / \rho(0)$, im Jahr 1993 [112, 113] hat man festgestellt, dass der magnetoresistive Effekt nahezu 100\% groß werden kann, deswegen der Begriff CMR (kolossaler Magnetowiderstand). Daran anschließende Untersuchungen haben zur Aufklärung vieler anderer ungewöhnlicher Eigenschaften der Manganite geführt, wie z.B. Ladungsordnung, Orbitalordnung, elektronische Phasenseparation u.s.w..

Die gemischtvalenten Manganite der Zusammensetzung $R_{1-x} D_{x} M n_{1-x}^{+3} M n_{x}^{+4} O_{3}\left(\mathrm{ABO}_{3}\right)$, wobei R ein trivalentes Kation aus der Lanthanoidenreihe $(\mathrm{R}=\mathrm{La}, \mathrm{Pr}, \mathrm{Nd}, \mathrm{Sm})$ und $\mathrm{D}$ ein divalentes Alkalimetall $(\mathrm{D}=\mathrm{Ca}, \mathrm{Sr}, \mathrm{Ba})$ ist, kristallisieren in verschiedenen Modifikationen der Perowskitstruktur. Das wesentliche Merkmal dieser Struktur ist die Anordnung von SauerstoffOktaedern, die das Mangan-Ion umgeben. In der idealen Perowskitstruktur (Abb. 1.2) sagen

\footnotetext{
${ }^{1}$ Das Mineral $\mathrm{CaTiO}_{3}$ ist nach dem Mineralogen V. Perowski benannt.
} 
geometrische Gründe für die Bindungslängen zwischen Position A $\left(\mathrm{Nd}^{+3}\right.$ oder Erdalkaliion, z.B. $\left.\mathrm{Sr}^{+2}\right)$ und Sauerstoff, $\mathrm{d}_{\mathrm{AO}}$, bzw. Position $\mathrm{B}\left(\mathrm{Mn}^{+3}, \mathrm{Mn}^{+4}\right)$ und Sauerstoff, $\mathrm{d}_{\mathrm{BO}}$, ein Verhältnis von $\mathrm{d}_{\mathrm{AO}} / \mathrm{d}_{\mathrm{BO}}=\sqrt{2}$ voraus. Geringfügige Abweichungen von diesem Wert führen zu einer Struktur mit geringerer Symmetrie. Ein Maß für solche Abweichungen von der Idealstruktur ist der Toleranzfaktor:

$$
T=\frac{1}{\sqrt{2}} \frac{d_{A O}}{d_{B O}} \approx \frac{1}{\sqrt{2}} \frac{R_{A}+R_{O}}{R_{B}+R_{O}},
$$

wobei $R_{A}, R_{B}$ die mittleren Ionenradien der eingebauten Ionen auf den Gitterplätzen A und $\mathrm{B}$ und $R_{O}$ der Radius des Sauerstoff-Ions sind.

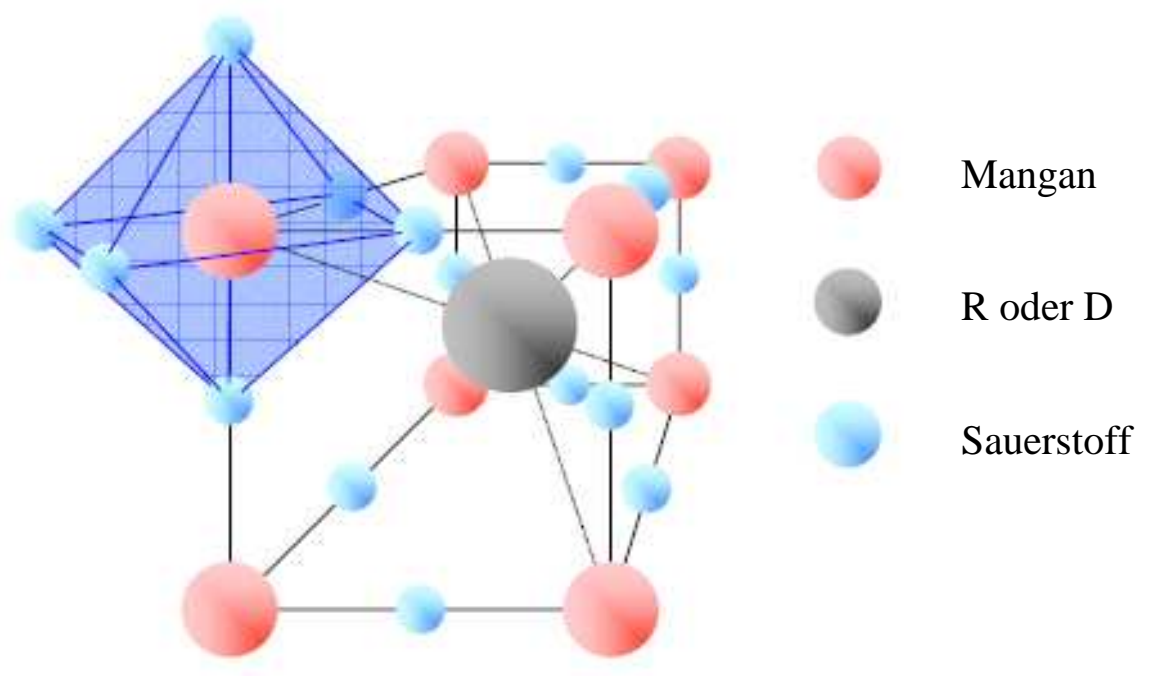

\section{Abbildung 1.2: Ideale Perowskitstruktur.}

Bei vielen Manganiten mit $\mathrm{T} \neq 1$ sind die negativ und positiv geladenen Ione immer noch symmetrisch verteilt, so dass keine spontane ferroelektrische Polarisation entsteht, und so sind die meisten physikalischen Eigenschaften dieser Manganite von der engen Verbindung des magnetischen Systems und des elektronischen Systems geprägt. Andererseits stellen Verbindungen mit Perowskitstruktur die größte Gruppe der Ferroelektrika vor. Das bekannteste Beispiel ist das Bariumtitanat $\left(\mathrm{BaTiO}_{3}\right)$, dessen Struktur unterhalb der ferroelektrischen CuriePunktes $\mathrm{T}_{\mathrm{C}}$ allerdings auch von der kubischen Symmetrie abweicht und dabei eine spontane ionische Polarisierung ausbildet: die $\mathrm{Ba}^{+2}$ und $\mathrm{Ti}^{+4}$-Ionen sind relativ $\mathrm{zu}$ den $\mathrm{O}^{-2}$-Ionen verschoben und bilden ein elektrisches Dipolmoment aus [106].

Materialien, die gleichzeitig die Eigenschaften von Ferroelektrika und Ferromagnetika aufweisen, werden „Ferroics“ genannt. Prototypen sind die perowskitähnlichen $\mathrm{RMn}_{2} \mathrm{O}_{5}$ Verbindungen ( $\mathrm{R}=$ Lanthanoidenion), deren formale Valenzproportion $\mathrm{Mn}^{+3} / \mathrm{Mn}^{+4} 1: 1$ ist. Oft lässt sich dort die gleichzeitige Existenz der elektrischen und magnetischen Dipolmomente [107] nachweisen, aber beide Untersysteme haben eine etwas andere Fernordnung ihrer 
Dipolmomente. In dem $\mathrm{EuMn}_{2} \mathrm{O}_{5 \pm \alpha^{-}}$System findet man z.B. eine kollineare spontane elektrische Polarisation bei Temperaturen unterhalb $40 \mathrm{~K}$ und eine helische Ordnung des Spinsystems. Die Natur der elektrischen Dipolmomente ist aber noch ungeklärt. Es werden Jahn-TellerVerzerrungen der $\mathrm{Mn}^{+3}$-Oktaedern oder ein Sauerstoffdefizit $\delta$ verantwortlich gemacht. $\delta$ kann auch eine Rolle bei der Entstehung von elektrischen Dipole spielen, durch eine Rekonfiguration der nächsten Nachbarschaft. In [108] und [111] wurden in diesem Zusammenhang auch lanthanoidendotierte PZT-Keramiken untersucht. Diese besitzen auch Perowskitstruktur und ferroelektrische Eigenschaften [109, 110]. Es wurde gezeigt, dass die Ferroelektrizität durch das Sauerstoffdefizit (Defekte) verstärkt oder geschwächt werden kann. Man muss also bei der Suche nach Substanzen, die sowohl magnetische Dipole als auch die elektrische Dipole entwickeln sollen, entweder Jahn-Teller-Systeme oder nicht stoichiometrische Systeme auswählen. Es ist auch $\mathrm{zu}$ erwarten, dass das magnetische System und das System der elektrischen Dipole auf irgendeine Art und Weise gekoppelt sind, denn die Ordnungstemperaturen fallen oft zusammen. In dieser Arbeit wurde ein sauerstoffdefizitärer Manganit $\mathrm{Nd}_{1-\mathrm{x}} \mathrm{Sr}_{\mathrm{x}} \mathrm{MnO}_{3-\delta}$ als Untersuchungsobjekt ausgewählt. 


\section{Experimentelles}

\subsection{Präparation der keramischen $\mathrm{Nd}_{2 / 3} \mathrm{Sr}_{1 / 3} \mathrm{MnO}_{3-\delta}$-Proben}

Bei der Herstellung der $\mathrm{Nd}_{2 / 3} \mathrm{Sr}_{1 / 3} \mathrm{MnO}_{3}$-Ausgangsproben wurde die übliche Keramiktechnik verwendet. Als Ausgangmaterialien haben wir die Oxide $\mathrm{Nd}_{2} \mathrm{O}_{3}, \mathrm{Mn}_{2} \mathrm{O}_{3}$ und das Karbonat $\mathrm{SrCO}_{3}$ genommen. Diese Substanzen wurden gewogen und in der gewünschten Proportion vermischt. Die Mischung wurde in einem Achatmörser bei gleichzeitiger Alkoholzugabe fein pulverisiert. Vor dem Abwiegen wurde das $\mathrm{Nd}_{2} \mathrm{O}_{3}$-Pulver ungefähr 2 Stunden bei $1000^{\circ} \mathrm{C}$ an Luft getempert, um die Feuchtigkeit $\left(\mathrm{H}_{2} \mathrm{O}\right)$ und das Kohlendioxid $\left(\mathrm{CO}_{2}\right)$ zu entfernen. Die vorbereitete Mischung aus zwei Oxiden und einem Karbonat wurde in Tabletten (Pellets) gepresst und für 2 Stunden an Luft bei $1100^{\circ} \mathrm{C}$ getempert. Danach wurden die Pellets wieder fein pulverisiert und das Pulver erneut in Pellets gepresst. Für die endgültige Synthese wurden die Pellets noch mal 2 Stunden an Luft bei $1500^{\circ} \mathrm{C}$ getempert. Um die Proben möglichst nahe der Stöchiometrie $\mathrm{zu}$ halten, wurden sie für 100 Stunden unter $900^{\circ} \mathrm{C}$ auf einer nicht reaktionsfreudigen Unterlage (in unserem Fall wurde ein Platinplättchen genommen) an Luft belassen. Danach wurden die Proben mit einer Rate von $100^{\circ} \mathrm{C} / \mathrm{h}$ bis auf Raumtemperatur abgekühlt.

Bei der Herstellung der sauerstoffdefizitären $\mathrm{Nd}_{2 / 3} \mathrm{Sr}_{1 / 3} \mathrm{MnO}_{3-\delta}$-Keramik wurde eine sogenannte topotaktische Reaktion verwendet. In diesem Vorgang wird die Probe zusammen mit einem Sauerstoffgetter (z.B. metallisches Tantal oder Vanadium) in ein evakuiertes Quarzrohr gebracht und bei einer bestimmten Temperatur getempert. In unserem Fall wurden die Proben für 24 Stunden in einem Ofen bei $900^{\circ} \mathrm{C}$ belassen. Als Sauerstoffgetter wurde metallisches Tantal verwendet. Nach 24 Stunden wurde das Quarzrohr mit den Proben langsam bis auf Raumtemperatur abgekühlt. Der Sauerstoffgehalt wurde aus dem Massenverlust der Proben bestimmt (man wiegt vor und nach der Reduktion). Um den relativen Fehler bei der Sauerstoffbestimmung zu limitieren, wurde als Probengewicht pro Ampulle nicht weniger als 2$3 \mathrm{~g}$ genommen. Wir haben damit den relativen Fehler auf $3 \%$ reduziert (der absolute Fehler $\Delta \delta$ ist kleiner als $0.025 / 10 /$ ). Nach der Sauerstoffreduktion wurde ein Teil der Proben reoxydiert (an Luft, $900^{\circ} \mathrm{C}, 24 \mathrm{~h}$ ). Nach der Reoxydation wurde die Massenzunahme bestimmt. Die Massenzunahme bei der Reoxydation stimmte mit dem Massenverlust bei der Reduktion überein.

\section{Probencharakterisierung}

\section{Röntgenbeugung}

Die Proben wurden mit einem Pulverdiffraktometer (DRON-3) im $\mathrm{Cr}-\mathrm{K}_{\alpha^{-}}$Modus und im Winkelintervall 20-100 untersucht. Die Röntgenspektren sind in Abbildung 2.3 dargestellt. Alle Proben zeigen eine orthorhombisch verzerrte Struktur (Raumgruppe Pbnm²). Die O'-Typ

\footnotetext{
${ }^{2}$ Die Pbnm-Raumgruppe ist der Pnma-Gruppe äquivalent. Die Basisvektoren a, b, c in Pbnm sind c, a, b in Pnma.
} 
orthorhombische Struktur ist in der Abbildung 2.1b dargestellt. Die elementare Zelle mit $a \approx \sqrt{2} a_{0}, b \approx \sqrt{2} a_{0}$ und $c \approx 2 a_{0}$ enthält vier Formeleinheiten $(\mathrm{Z}=4)$. Diese Struktur ist bei den $\mathrm{RMnO}_{3}$-Verbindungen $(\mathrm{R}=\mathrm{La}-\mathrm{Ca})$ oft zu finden [81]. Die Verzerrung ist im wesentlichen durch den Jahn-Teller-Effekt verursacht [82]. Von fremden Phasen in der Probe wurden keine Spuren gefunden. Wir können aber die Anwesenheit eindiffundierten Tantals nicht ausschließen (vermutlich vorwiegend im Bereich der Korngrenzen). Die vom Auswertungsprogramm erhaltenen Werte für die Gitterkonstanten sind in Tabelle 2.1 zusammengefasst. Die Werte der Gitterkonstanten stimmen mit den Literaturdaten gut überein [73].

\begin{tabular}{c|c|c|c|c}
$\begin{array}{c}\text { Probe } \\
\mathbf{N d}_{2 / 3} \mathbf{S r}_{\mathbf{1} 3 \mathbf{3}} \mathbf{M n O}_{\mathbf{3}-\boldsymbol{\delta}}\end{array}$ & Raumgruppe & $\mathbf{a}(\AA)$ & $\mathbf{b}(\AA)$ & $\mathbf{c}(\AA)$ \\
\hline $\boldsymbol{\delta}=\mathbf{0 . 2 0}$ & P b n m & $5.48230(113)$ & $5.48241(00)$ & $7.75117(164)$ \\
\hline $\boldsymbol{\delta}=\mathbf{0 . 1 5}$ & P b n m & $5.47368(36)$ & $5.46549(40)$ & $7.74841(47)$ \\
\hline $\boldsymbol{\delta}=\mathbf{0 . 1 0}$ & P b n m & $5.46411(27)$ & $5.45147(32)$ & $7.73016(43)$ \\
\hline $\boldsymbol{\delta}=\mathbf{0 . 0 5}$ & P b n m & $5.46445(23)$ & $5.44879(26)$ & $7.70825(37)$ \\
\hline $\boldsymbol{\delta}=\mathbf{0}$ & P b n m & $5.44759(25)$ & $5.46351(25)$ & $7.69733(37)$
\end{tabular}

Tabelle 2.1: Gitterkonstanten von $\mathrm{Nd}_{2 / 3} \mathrm{Sr}_{1 / 3} \mathrm{MnO}_{3-\delta}$

Der Abbildung 2.1a ist auch die Abhängigkeit des Volumes ( $\mathrm{V}=\mathrm{a}^{*} \mathrm{~b}^{*} \mathrm{c}$ ) einer Elementarzelle zu entnehmen. Die Daten zeigen eine Anomalie (Änderung der Steigung in der V( $\delta$ )-Kurve) bei $\delta \approx$ 0.078, sodass man bei der Variation des Sauerstoffgehaltes zwei verschiedenen Regime vermuten kann. In der Abbildung 2.2 werden die Gitterparameter selbst grafisch dargestellt. Die Anomalie ist auch hier zu erkennen.
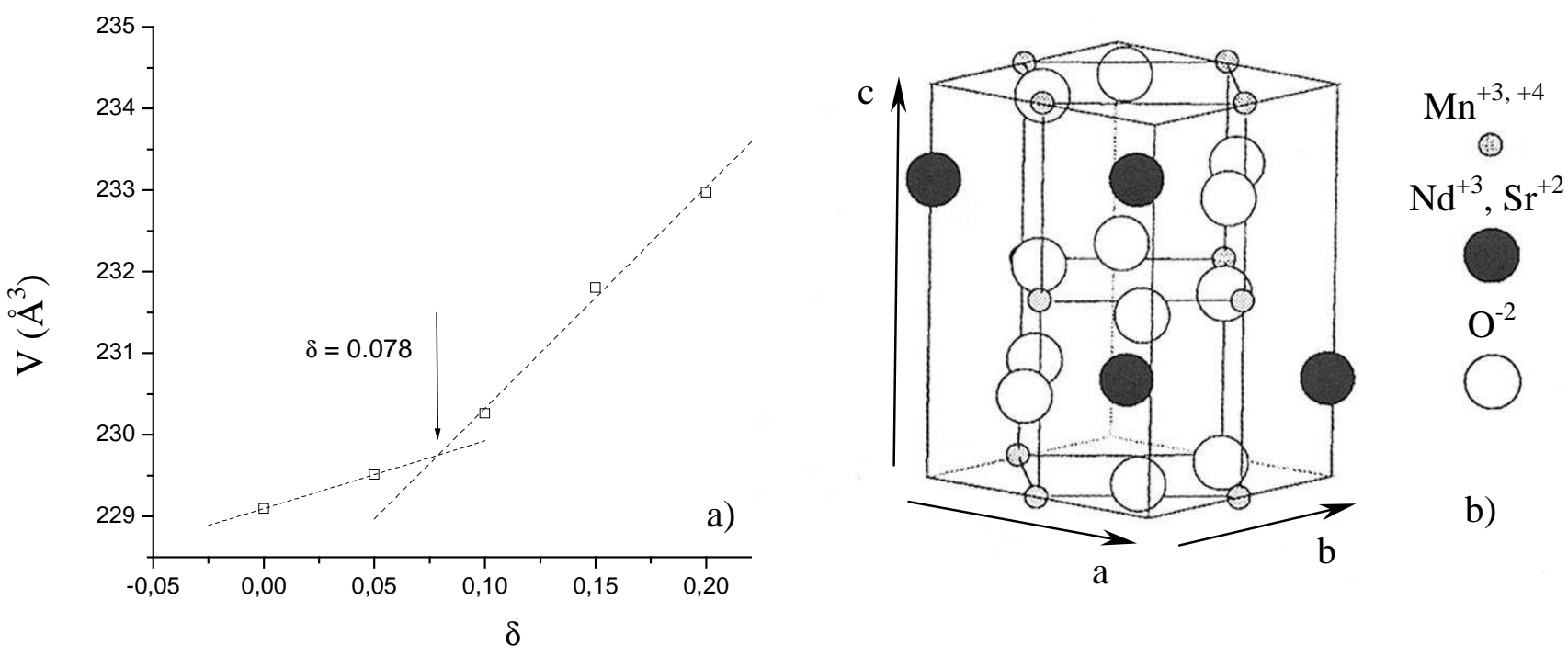

b)

Abbildung 2.1: a) Das Volumen $\left(V=a^{*} b^{*}\right.$ c) der Elementarzelle bei den verschiedenen $\left.\delta . b\right)$ die Einheitszelle des $O^{\prime}$-Typ orthorhombisch verzerrten Kristallgitter. 

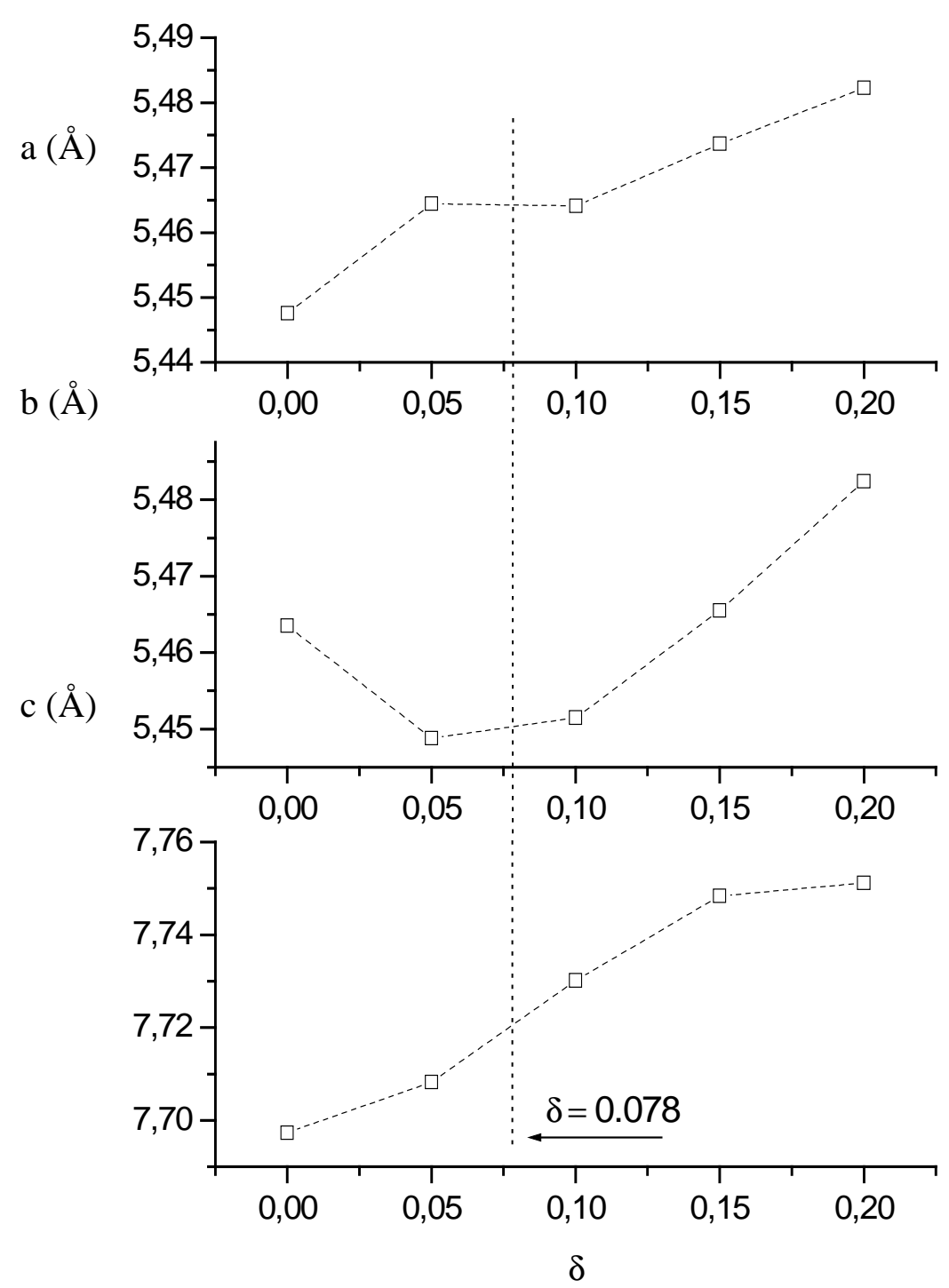

Abbildung 2.2: Die Gitterkonstanten a, b, c von $\mathrm{Nd}_{2 / 3} \mathrm{Sr}_{1 / 3} \mathrm{MnO}_{3-\delta}$ in Abhängigkeit vom Sauerstoffdefizit $\delta$. 


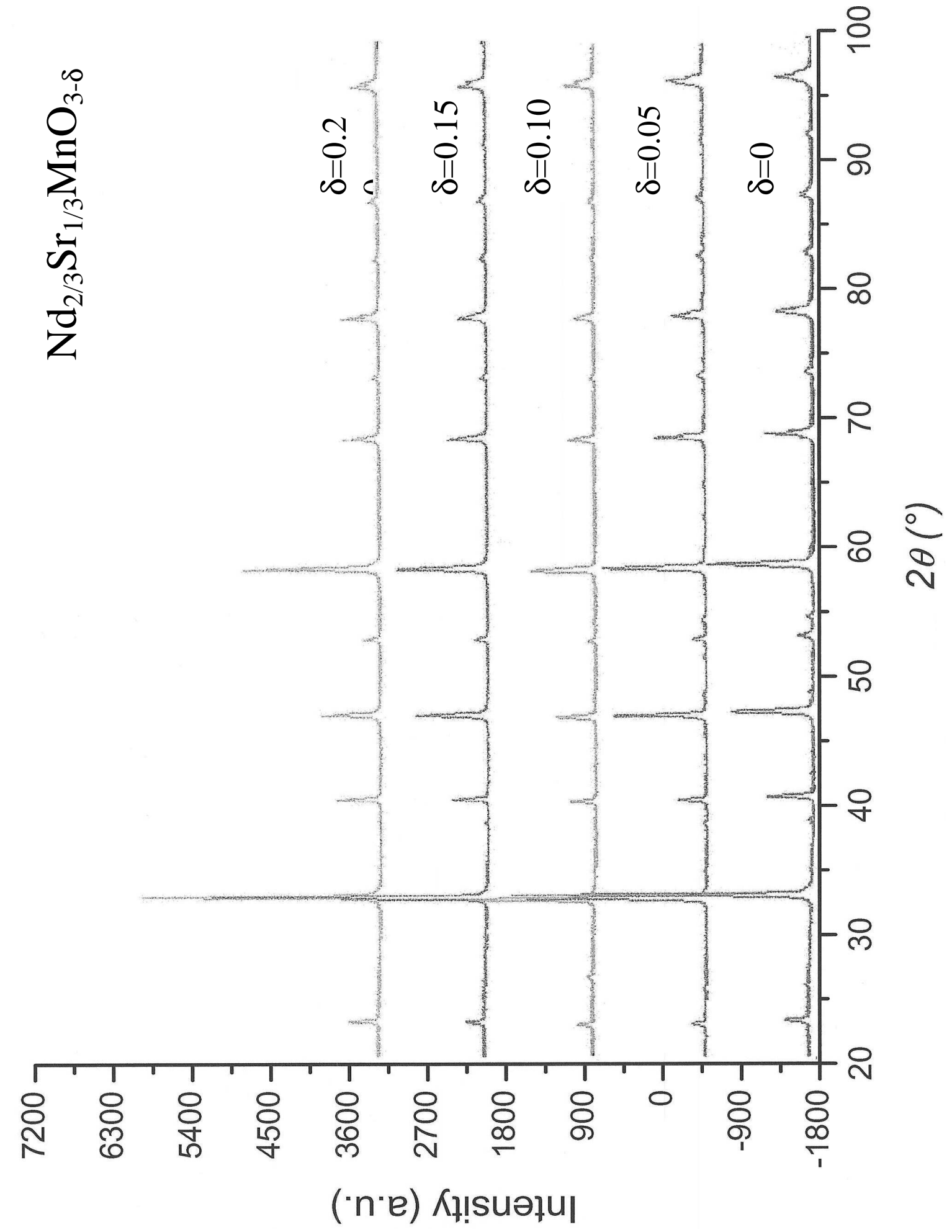

Abbildung 2.3: Pulverdiffraktogramme der $\mathrm{Nd}_{2 / 3} \mathrm{Sr}_{1 / 3} \mathrm{MnO}_{3-\delta}$ Keramik. 


\section{Rasterelektronenmikroskopie (SEM)}

Die stoichiometrischen Proben $\mathrm{Nd}_{2 / 3} \mathrm{Sr}_{1 / 3} \mathrm{MnO}_{3}$ wurden mit Hilfe der Rasterelektronenmikroskopie untersucht ${ }^{3}$. Dabei wurde das Rasterelektronenmikroskop CamScan-4 mit einer Wolframkathode (Fa. Cambridge Ltd.) verwendet. In der Abbildung 2.4 ist ein SEM-Bild einer der keramischen $\mathrm{Nd}_{2 / 3} \mathrm{Sr}_{1 / 3} \mathrm{MnO}_{3}$-Probe zu sehen.

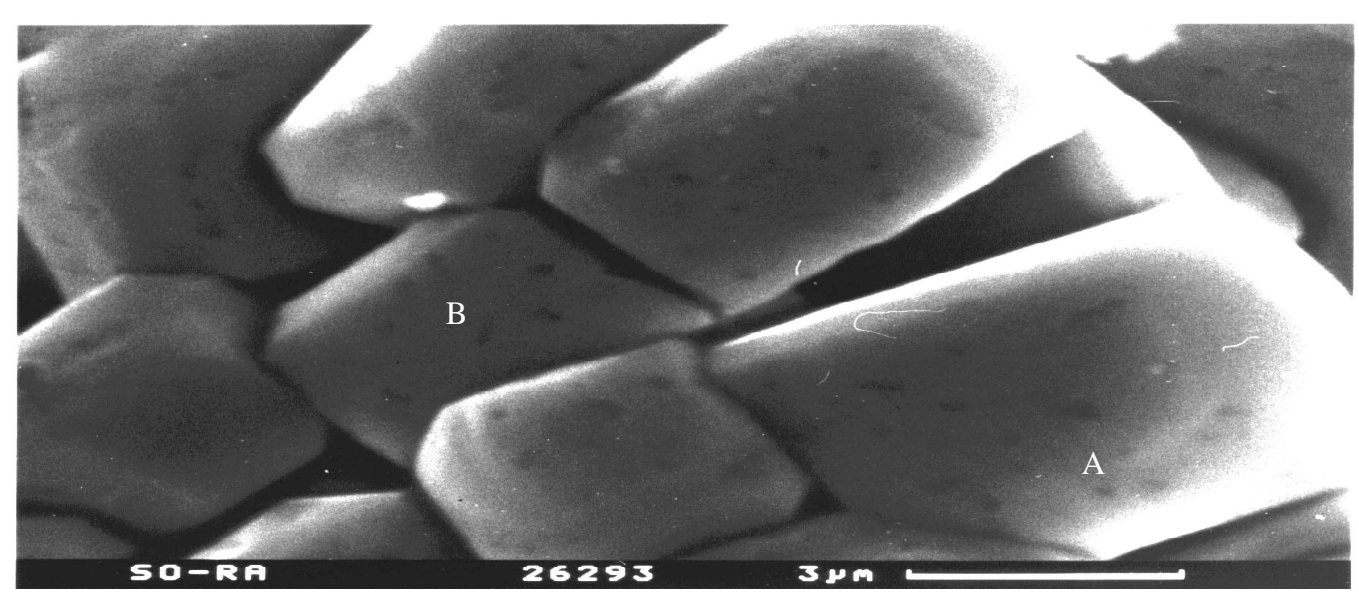

Abbildung 2.4: SEM-Bild von einer $\mathrm{Nd}_{2 / 3} \mathrm{Sr}_{1 / 3} \mathrm{MnO}_{3}$-Keramik.

Um die Zusammensetzung der Probe besser zu charakterisieren, wurde zusätzlich die Erweiterung des Mikroskops für eine Röntgenfluoreszenzuntersuchung (energy dispersive X-ray microanalysis system „Voyager“ mit einem $\mathrm{Si}(\mathrm{Li})$-Detektor und mit einem ultra dünnen Fenster der Fa. „Thermo Noran“, USA) benutzt. In der Abbildung 2.5 sind die Röntgenfluoreszenzdiffraktogramme für die Kristallite A und B gezeigt (siehe Abbildung 2.4).

Auch diese Untersuchungen haben ergeben, dass die $\mathrm{Nd}_{2 / 3} \mathrm{Sr}_{1 / 3} \mathrm{MnO}_{3}$-Probe keine fremde Phasen enthält: die Röntgenfluoreszenzdiffraktogramme sind für beide untersuchten Kristallite gleich. Die Röntgenbeugung hat ebenso gezeigt, dass die Probe homogen ist (siehe Abbildung 2.3).

\footnotetext{
${ }^{3}$ Für diese Messungen sind wir U. Sondermann (Philipps-Universität, Marburg) dankbar.
} 

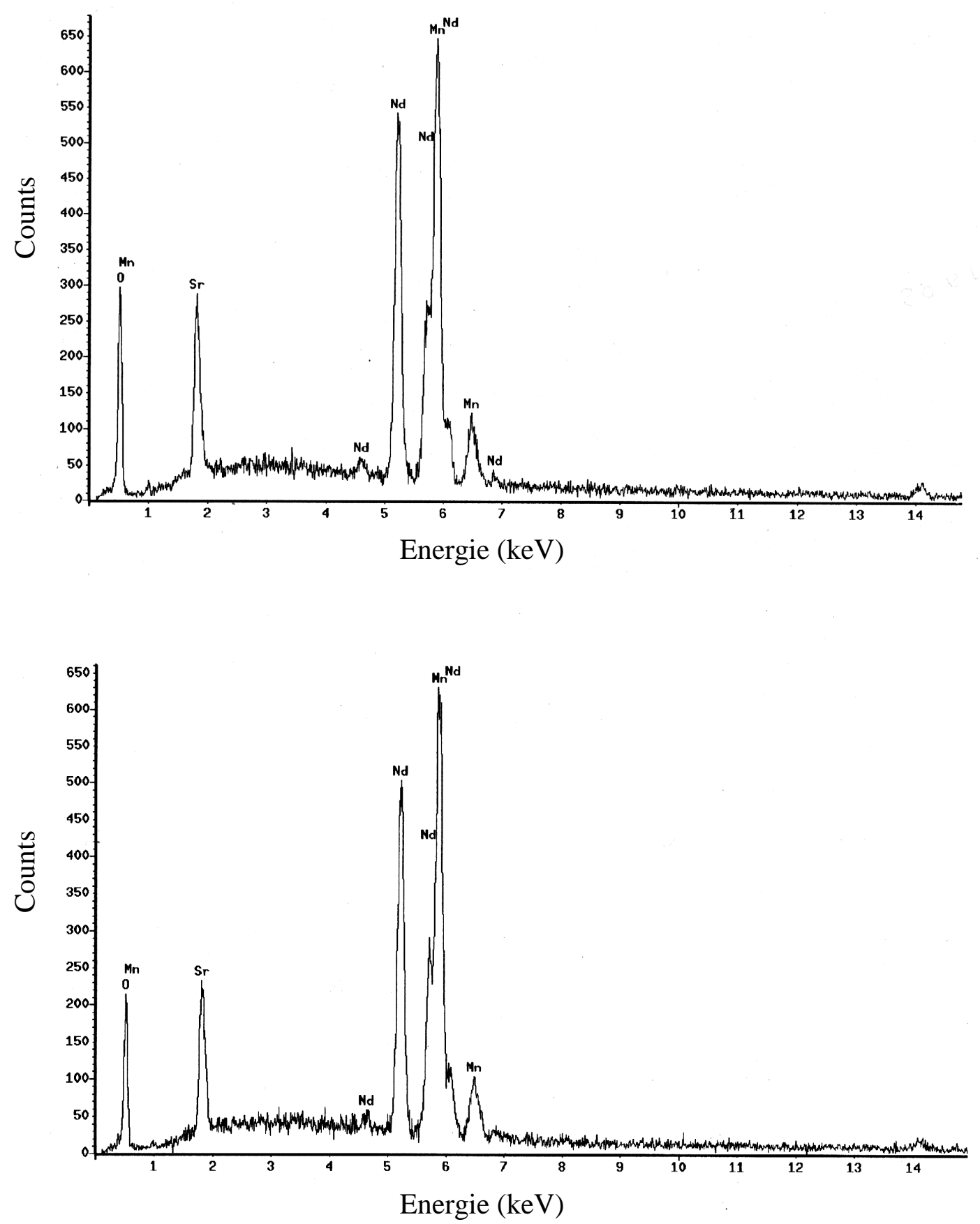

Abbildung 2.5: Die Röntgenfluoreszenzdiffraktogramme von zwei verschieden Kristalliten der stoichiometrischen $\mathrm{Nd}_{2 / 3} \mathrm{Sr}_{1 / 3} \mathrm{MnO}_{3}$-Probe (oben: Kristallit A, unten: Kristallit B)

\section{Transmissionselektronenmikroskopie (TEM)}

Die Probe mit dem größten Sauerstoffdefizit $\delta=0.20$ wurde zusätzlich mit Hilfe der Transmissionselektronenmikroskopie (TEM) charakterisiert. Wir haben insbesondere versucht, die Korngrenzen der Probe zu finden. In der Abbildung 2.6 ist ein in der Nähe einer Korngrenze aufgenommenes Diffraktogramm gezeigt. Man erkennt hier, dass die Beugung von zwei benachbarten Kristalliten herrührt, die verschieden orientiert sind. 


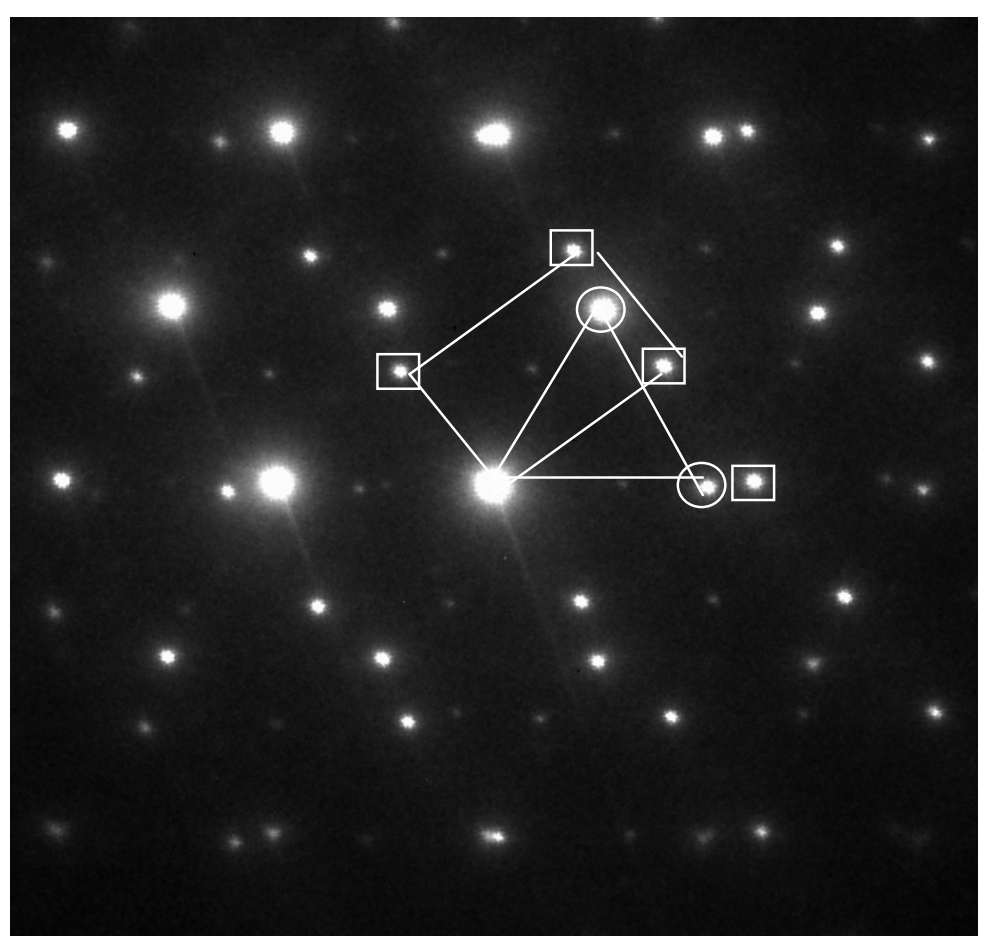

Abbildung 2.6: TEM-Beugung von zwei benachbarten Kristalliten (A und B). $\square$ und $\mathrm{O}$ selektieren die Reflexe aus $A$ und $B$.

Die Abbildungen 2.7 und 2.8 zeigen je ein TEM-Bild von einer Korngrenze. Das Bild 2.7 zeigt insbesondere eine Grenze zwischen einem Kristallit und einem amorphen Bereich. Im zweiten Fall (Bild 2.8) ist eine Grenze zwischen zwei benachbarten Kristalliten abgebildet.

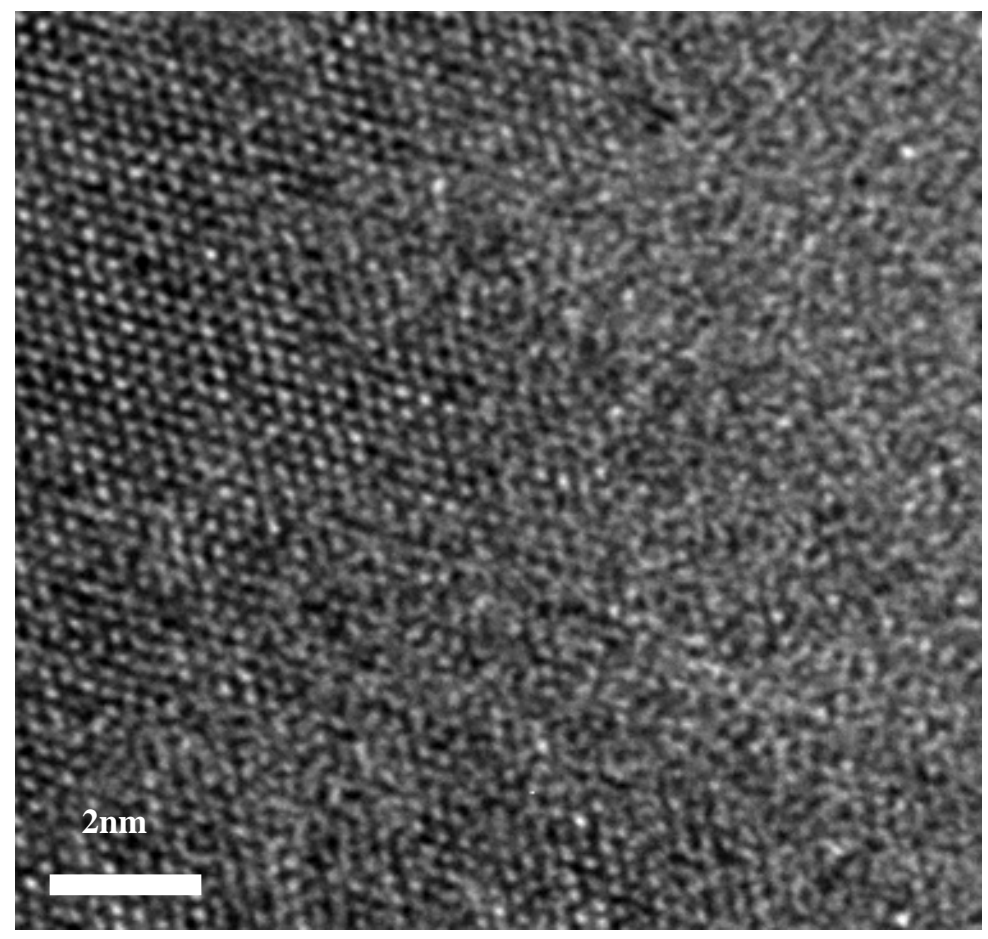

Abbildung 2.7: Der geordnete Teil des Bildes entspricht einem Kristallit und der verschmierte Bildrest zeigt eine amorphe Phase. 


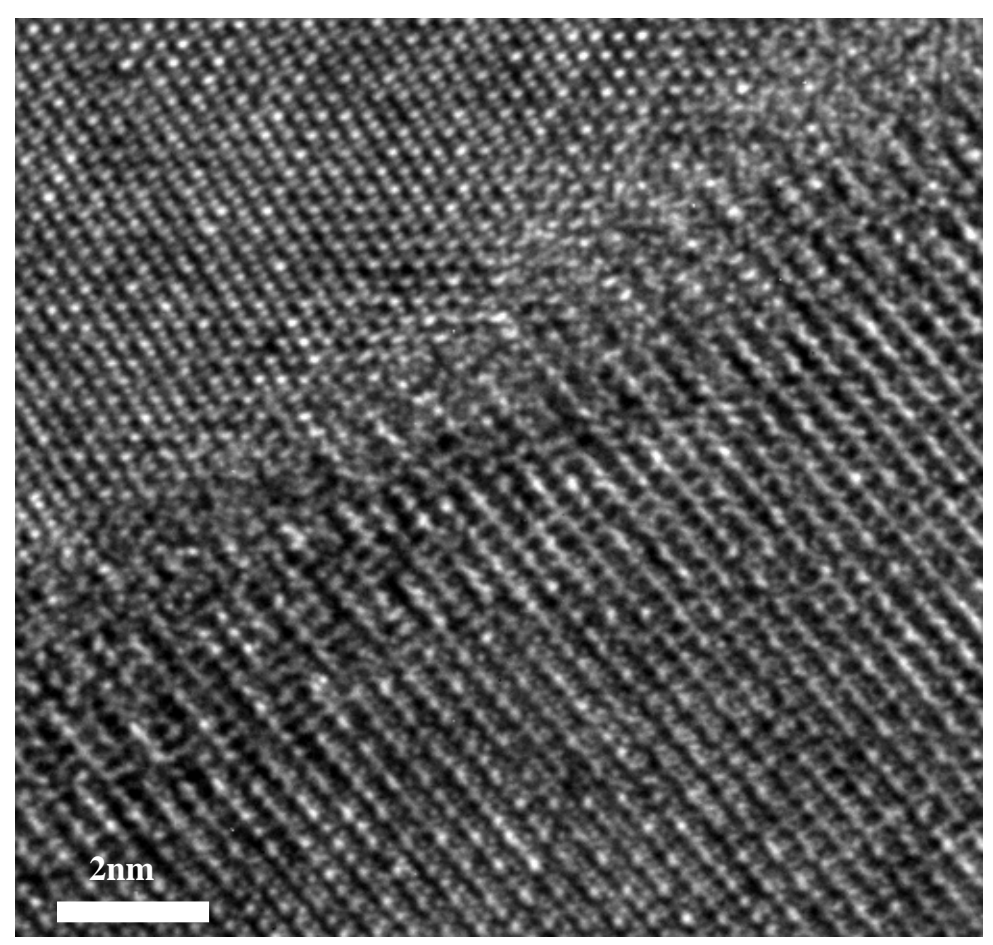

Abbildung 2.8: Man sieht hier eine diffuse Grenze zwischen zwei Kristalliten unterschiedlicher Orientierung.

\subsection{Präparation und Charakterisierung der $\mathrm{Nd}_{2 / 3} \mathrm{Sr}_{1 / 3} \mathrm{MnO}_{3-\delta}-\mathrm{Schichtproben.}$}

$\mathrm{Nd}_{2 / 3} \mathrm{Sr}_{1 / 3} \mathrm{MnO}_{3-\delta^{-}}$Schichtproben wurden mit Hilfe der PLD-Methode (pulsed laser deposition) hergestellt ${ }^{4}$. Die detaillierte Beschreibung dieser Methode ist zum Beispiel in [21] zu finden. Dabei wurde ein KrF-Laser (Fa. „, $\lambda$-Physik“) mit der Wellenlänge $\lambda=248 \mathrm{~nm}$ verwendet. Die mittlere Energiedichte des Laserstrahles bei der Probenpräparation beträgt $2 \mathrm{~J} / \mathrm{cm}^{2}$. Der Laser gab die Strahlungspulse mit einer Frequenz von $\mathrm{f}=3 \mathrm{~Hz}$ aus. Die Depositionszeit $t_{\mathrm{D}}$ betrug bei allen Schichten ungefähr $45 \mathrm{~min}$ (8000 Pulse). Als Target wurde eine keramische $\mathrm{Nd}_{2 / 3} \mathrm{Sr}_{1 / 3} \mathrm{MnO}_{3}$-Probe (hergestellt nach der üblichen Keramikmethode, siehe Unterkapitel: „Präparation der keramischen $\mathrm{Nd}_{2 / 3} \mathrm{Sr}_{1 / 3} \mathrm{MnO}_{3-\delta}$-Proben“) benutzt. Die Schichten wurden auf einem $5 \times 5 \times 1 \mathrm{~mm}$ breiten (100) $\mathrm{SrTiO}_{3}$-Substrat (kubisch, mit a = $3.905 \AA$, hergestellt von der Fa. „Alfa Aesar") deponiert. Das Ziel war epitaktisches Wachstum. Die Distanz zwischen dem Target und dem Substrat betrug bei allen Proben $5 \mathrm{~cm}$. Um eine Probenserie mit verschiedenen Sauerstoffdefizit $\delta \mathrm{zu}$ bekommen, wurden die Schichten in einer gemischten $\left(\mathrm{O}_{2} / \mathrm{Ar}\right)$ Atmosphäre präpariert (dabei wurde der Partialdruck des Sauerstoffes in der Präparationskammer während der Präparationszeit und während der Abkühlung der Probe variiert). Der Gesamtdruck in der Kamera blieb aber während des Wachstums der Schichten konstant ( $\mathrm{p}=0.3$ mbar). Zusätzlich wurde die Substrattemperatur variiert, um die beste Qualität der Schichten zu erreichen. Wir haben festgestellt, dass die optimale Temperatur des Substrates während der Depositionszeit $620^{\circ} \mathrm{C}$ ist.

\footnotetext{
${ }^{4}$ Dafür sind wir B. Mercey und B. Raveau (ISMRA et Université de Caen, France) dankbar.
} 
In dieser Arbeit wird eine Serie von vier Schichtproben mit unterschiedlichem Sauerstoffdefizit $\delta$ diskutiert. Die Präparationsbedingungen sind in der Tabelle 2.2 zusammengefasst.

\begin{tabular}{|l|c|c|c|c|}
\hline & Probe A & Probe B & Probe C & Probe D \\
\hline Substratstemperatur & $620^{\circ} \mathrm{C}$ & $620^{\circ} \mathrm{C}$ & $620^{\circ} \mathrm{C}$ & $620^{\circ} \mathrm{C}$ \\
\hline $\mathrm{O}_{2}$-Partialdruck & $0.1 \mathrm{mbar}$ & $0.03 \mathrm{mbar}$ & $0.015 \mathrm{mbar}$ & $0.015 \mathrm{mbar}$ \\
\hline Ar-Partialdruck & $0.2 \mathrm{mbar}$ & $0.27 \mathrm{mbar}$ & $0.285 \mathrm{mbar}$ & $0.285 \mathrm{mbar}$ \\
\hline $\mathrm{O}_{2}$-Druck während der Abkühlung & $200 \mathrm{mbar}$ & $100 \mathrm{mbar}$ & $100 \mathrm{mbar}$ & $\begin{array}{c}\text { Wie bei der } \\
\text { Präparationszeit }\end{array}$ \\
\hline Strahlenergie & $216 \mathrm{~mJ}$ & $214 \mathrm{~mJ}$ & $219 \mathrm{~mJ}$ & $215 \mathrm{~mJ}$ \\
\hline
\end{tabular}

Tabelle 2.2: Die Präparationsbedingungen für die sauerstoffdefizitären $\mathrm{Nd}_{2 / 3} \mathrm{Sr}_{1 / 3} \mathrm{MnO}_{3-\delta}$ Schichten.
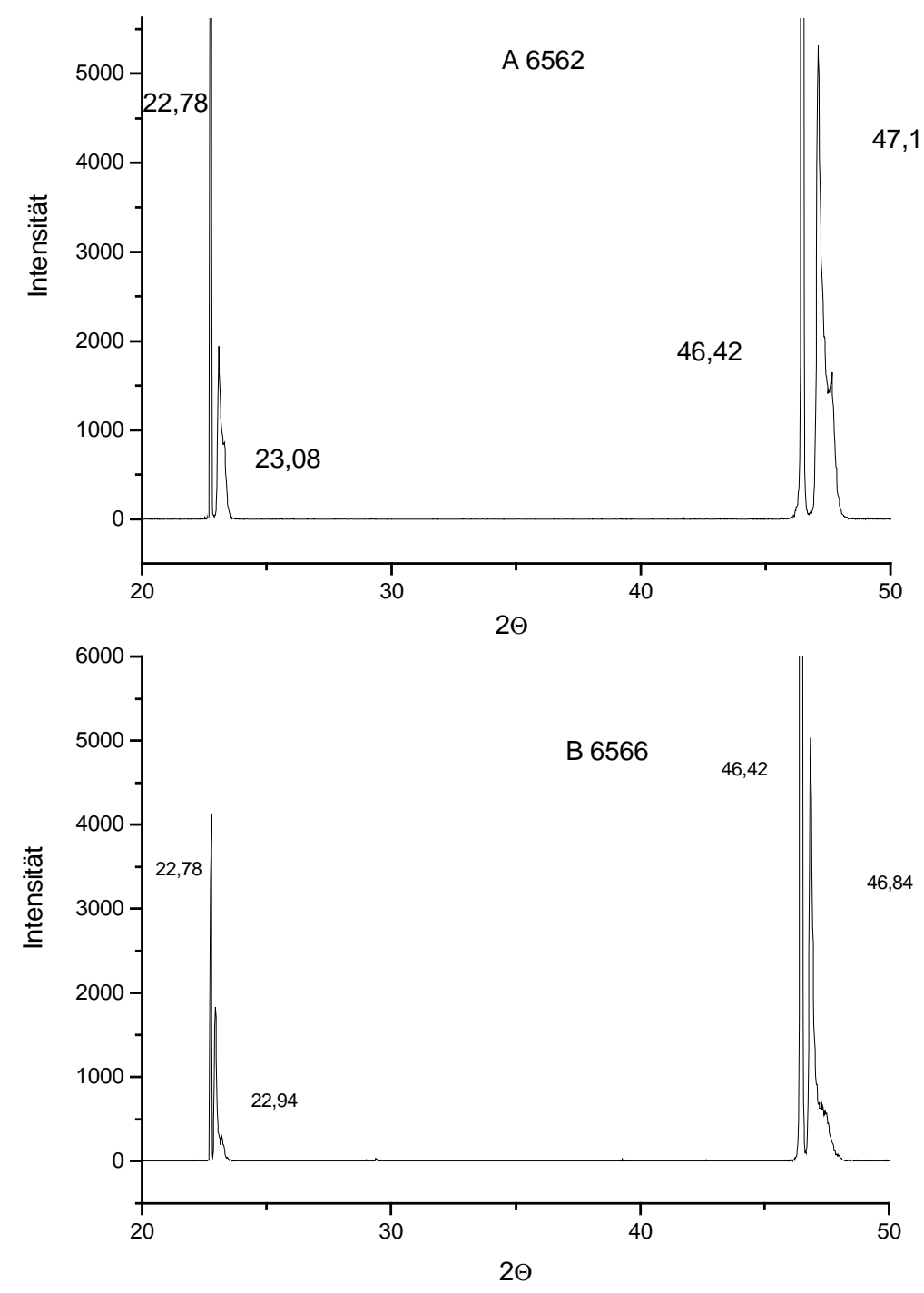

Abbildung 2.9: Röntgendiffraktogramme der $\mathrm{Nd}_{2 / 3} \mathrm{Sr}_{1 / 3} \mathrm{MnO}_{3-\delta}$ Schichtproben ( $\delta \approx 0$ und 0.02). 
In der Abbildung 2.9 sind die Röntgendiffraktogramme von zwei Schichten (A und B) gezeigt. Auf dem Diffraktogramm sieht man, dass die zwei Spitzen, die der Schicht zugeordnet werden müssen (das rechte Maximum von jedem Duplett), nach rechts etwas verbreitert sind, was wohl auf Verspannungen im Grenzbereich zwischen Substrat und Schicht hinweist.

\subsection{Messaufbauten}

\section{Die Faraday-Methode zur Messung der Magnetisierung}

Die Magnetisierungsmessungen an unseren Proben wurden in einem Temperaturintervall zwischen $80 \mathrm{~K}$ und Raumtemperatur und im Magnetfeld (0.25T) durchgeführt. Dabei wurde die Faraday-Methode verwendet.

Die Faraday-Methode ist eine Standardmethode für die Untersuchung von magnetischen Materialien. Wenn eine Probe mit dem magnetischen Moment $\mathbf{M}$ sich in einem inhomogenen Magnetfeld befindet, dann wirkt auf diese Probe eine Kraft F, die proportional zu M ist. Diese Kraft lässt sich aus der Zeemann-Energie W bestimmen:

$\mathrm{W}=-\mathbf{M}^{*} \mathbf{H}$

daraus folgt für die Kraft:

$\mathrm{F}=-\operatorname{grad}(\mathrm{W})=\operatorname{grad}\left(\mathbf{M}^{*} \mathbf{H}\right)$

In unserem Aufbau (sehe Abbildung 2.10) erzeugen die Polschuhe einen Gradienten in zRichtung, der eine Kraft in dieser Richtung erzeugt. In diesem Fall ergibt sich:

$F_{z}=-d W / d z=d\left(H_{x} * M_{x}\right) / d z$

Für eine punktförmige Probe ergibt sich insbesondere:

$\mathrm{F}_{\mathrm{z}}=\mathrm{M}_{\mathrm{x}} *\left(\mathrm{dH} \mathrm{H}_{\mathrm{x}} / \mathrm{dz}\right)$

Bei ausgedehnten Proben wird ein mittleres $M_{x}$ angesetzt.

Die Messapparatur bestand aus einem selbstgewickelten Elektromagneten mit abgeschrägten Polschuhen als Ersatz für ein separates Gradientenspulensystem (Abbildung 2.10). Die von der Waagenelektronik ausgegebenen Messpunkte wurden mit Hilfe eines digitalen Multimeters (Fa. Keithley) abgegriffen und direkt im Computer gespeichert. Die Messungen wurden bei einem maximalen Magnetfeld von $\mathrm{B}=0.25 \mathrm{~T}$ im Temperaturbereich $80 \mathrm{~K}<\mathrm{T}<300 \mathrm{~K}$ durchgeführt. Die Auflösungsgrenze der verwendeten Waage (Fa. Sartorius) liegt bei $10^{-3} \mathrm{~g}$, sodass zusammen mit dem Fehler bei der Probeneinwaage $(\approx 1 \%)$ und der Ungenauigkeit bei der Bestimmung von 
$\mathrm{dB} / \mathrm{dz}(\approx 10 \%)$ der Absolutwertfehler für die Messung $\mathrm{zu} \leq 15 \%$ begrenzt werden kann. Die Relativwerte sind genauer, ca. $2 \%$.

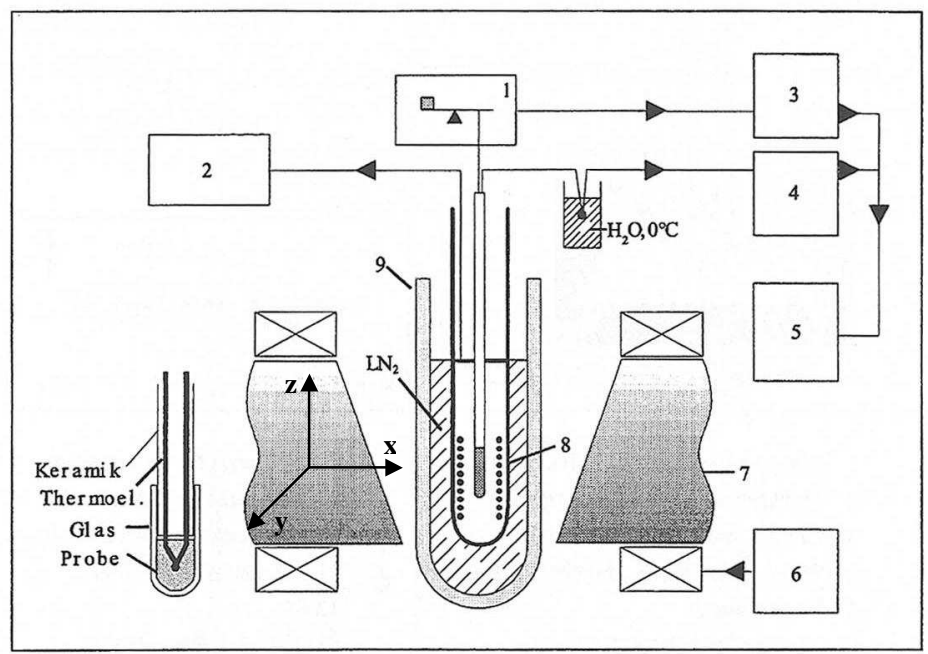
1. Milligrammwaage (Fa. Sartorius)
6. Netzgerät für Elektromagnet
2. Netzgerät für Heizung
7. Elektromagnet
3. Waagenelektronik
8. Quarzglasrohr
4. Digitalmultimeter
9. Dewar
5. Datenerfassung

Abbildung 2.10: Blockschaltbild des Faradaymagnetometers.

\section{Spezifische Wärmekapazität}

Für die Messungen der spezifischen Wärmekapazität aller in dieser Arbeit untersuchten $\mathrm{Nd}_{2 / 3} \mathrm{Sr}_{1 / 3} \mathrm{MnO}_{3}$-Proben wurde ein differentielles Kalorimeter (Perkin Elmer DSC-2) verwendet ${ }^{5}$.

\section{Spezifischer Widerstand}

Der spezifische Widerstand $\rho$ wurde nach der 4-Punkt-Methode unter konstantem Strom gemessen. Die Proben wurden in einem $\mathrm{N}_{2}$-Badkryostaten untergebracht. Mit dem Kryostat konnten wir den Widerstand im Bereich von $77 \mathrm{~K}$ bis Raumtemperatur quasi-statisch (d.h. bei einer Heizrate von $\mathrm{dT} / \mathrm{d} t \approx 1 \mathrm{~K} / \mathrm{min}$ ) messen. Die Proben, die in quaderförmiger Form $(7 \mathrm{~mm} * 3 \mathrm{~mm} * 2 \mathrm{~mm})$ geschnitten waren, wurden mit Hilfe von Leitsilber 200 (Fa. „Hans Wolbring“) kontaktiert. Für die Spannungsmessung wurde ein Multimeter (Keithley 2000) benutzt. Der konstante Strom wurde mit Hilfe einer einstellbaren Konstantstromquelle (Knick J 400) erzeugt. Um dem Fall, dass die Impedanz der Quelle und der Probe nicht mehr angepasst sind, zu entgehen, wurde der durch die Probe fließende Strom ständig beobachtet. Die Spannung wurde zwischen den zwei inneren Kontakten (Spannungsmodus des Keithley-Multimeters) mit einer Eingangsimpedanz von 1G $\Omega$ gemessen. Mit einem Pt100-Widerstand, der mit Wärmeleitpaste direkt an die Probe angeklebt wurde, konnte die Probentemperatur bis auf etwa $1 \mathrm{~K}$ genau bestimmt werden. Der größte Anteil am Gesamtfehler des Absolutwertes ist dem

\footnotetext{
${ }^{5}$ Mit freundlicher Unterstützung von Herrn Prof. Gmelin vom Max-Planck-Institut in Stuttgart durchgeführt.
} 
Fehler zuzuschreiben, der bei der Bestimmung der Probenabmessung gemacht wird: bei den obengenannten Probenmaßen muss dieser Fehler mit $\leq 10 \%$ geschätzt werden. Der relative Fehler ist aber deutlich geringer, ca $1 \%$.

\section{Bestimmung der Wärmeleitfähigkeit $K(T)$ und der Thermokraft $S(T)$ durch die Methode der zeitaufgelösten thermoelektrischen Effekte (TTE)}

Die TTE-Methode (TTE = transient thermoelctric effects) wurde von M. Sasaki, H. Negishi und M. Inoue zur Untersuchung von n- und p-dotiertem Silizium entwickelt. Seit dieser Zeit (1986) wurde diese Methode zur Untersuchung vieler verschiedener Materialien eingesetzt. Eine gute Referenzsammlung, die eine Übersicht über das Arbeitsfeld der TTE-Methode gibt, ist in [2] zu finden.

Wir benutzen diese Methode hier lediglich zur Bestimmung von Thermokraft und Wärmeleitfähigkeit. Die Methode erlaubt es wegen einer räumlich und zeitlich begrenzten Beleuchtung und dem daraus folgendem lokalen Aufheizen der Probe die Thermospannung für halbleitende und metallische Proben abzugreifen. Insbesondere folgt aus der Anfangsamplitude dieses TTE-Transienten die Thermokraft $\mathrm{S}$ und aus dem Relaxationsverhalten die Wärmediffusionskonstante $\kappa$.

Den Thermodiffusionstransienten sieht man beispielweise in Abbildung 2.11. Das TTE-Signal einer einkristallinen $\operatorname{Pr}_{0,63} \mathrm{Ca}_{0,37} \mathrm{MnO}_{3}$-Probe zeigt hier ein aus zwei Bereichen bestehendes Abklingverhalten. Aufgrund der sich im Vorzeichen unterscheidenden Anfangsspannungen und der unterschiedlichen Zeitskalen lassen sich in diesem Beispiel die beiden Beiträge gut trennen. Der langsam abfallende Teil ist die Thermodiffusion, während der schnell abfallende Teil der Relaxation einer Raumladung zugeordnet wird [117].

In Abbildung 2.12 wird der experimentelle Aufbau zur TTE-Messung schematisch dargestellt. Als optische Anregungsquelle diente eine 1000 W - Halogenlampe mit Reflektor. Das Halogenlampenspektrum ist in Abbildung $2.13 \mathrm{zu}$ sehen. Das Spektrum wurde im Bereich 430$730 \mathrm{~nm}$ vermessen. Bei der Messung wurde ein Monochromator „Spex 270M“ und eine CCDKamera $(1024 x 128)$ von „SpectrumONE“ verwendet. Direkt an der Probe ist aber ein durch das Quarzglas des Kryostatfensters leicht geändertes Spektrum zu erwarten. 


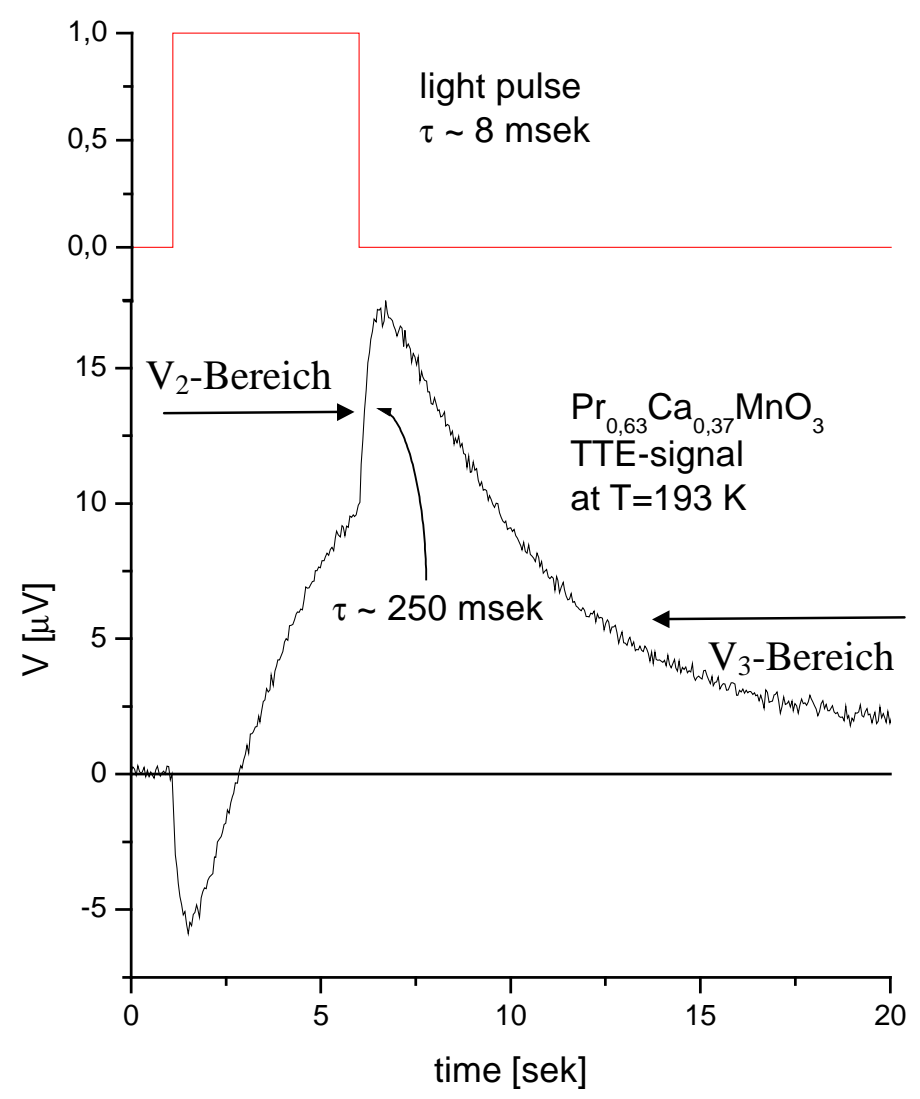

Abbildung 2.11: Eine typische TTE-Kurve; hier am Beispiel der einkristallinen $\mathrm{Pr}_{0,63} \mathrm{Ca}_{0,37} \mathrm{MnO}_{3}$-Probe. Oben wurde die Lichtanregung mit der Abklingzeit $\tau \approx 8$ msek aufgezeichnet.

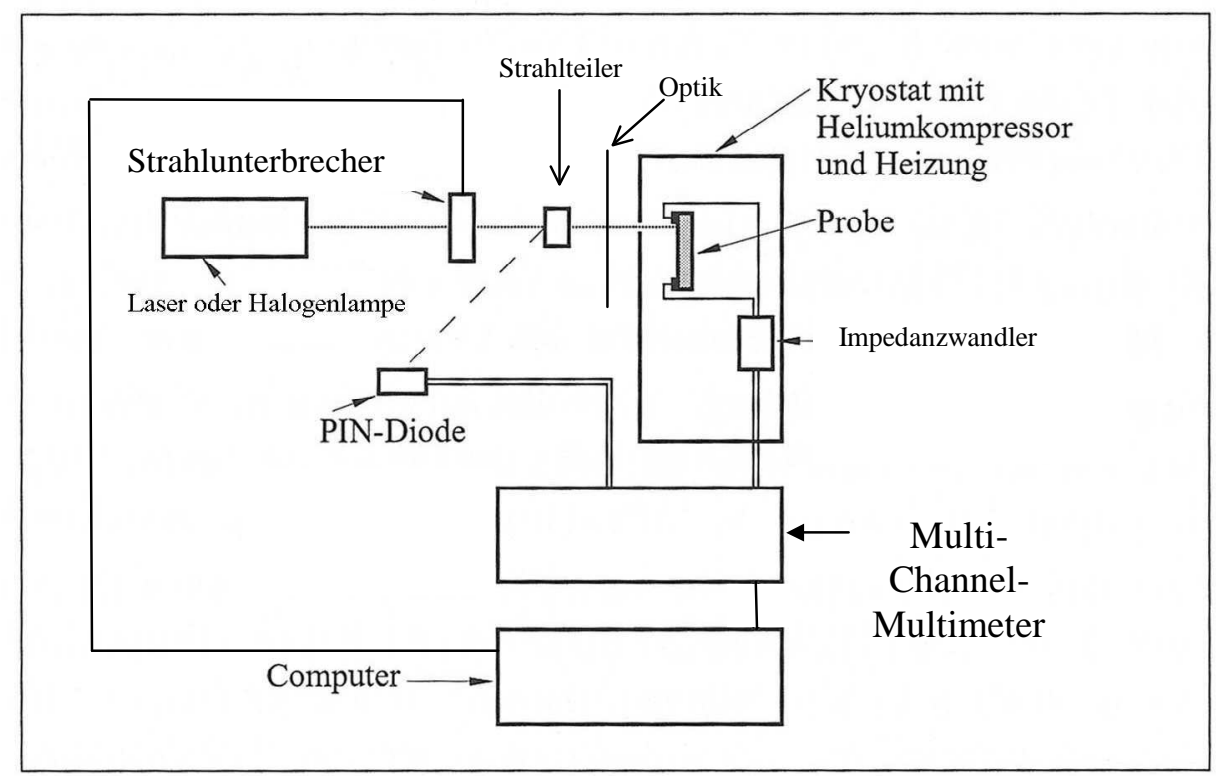

Abbildung 2.12: Experimenteller Aufbau zur TTE-Messung 


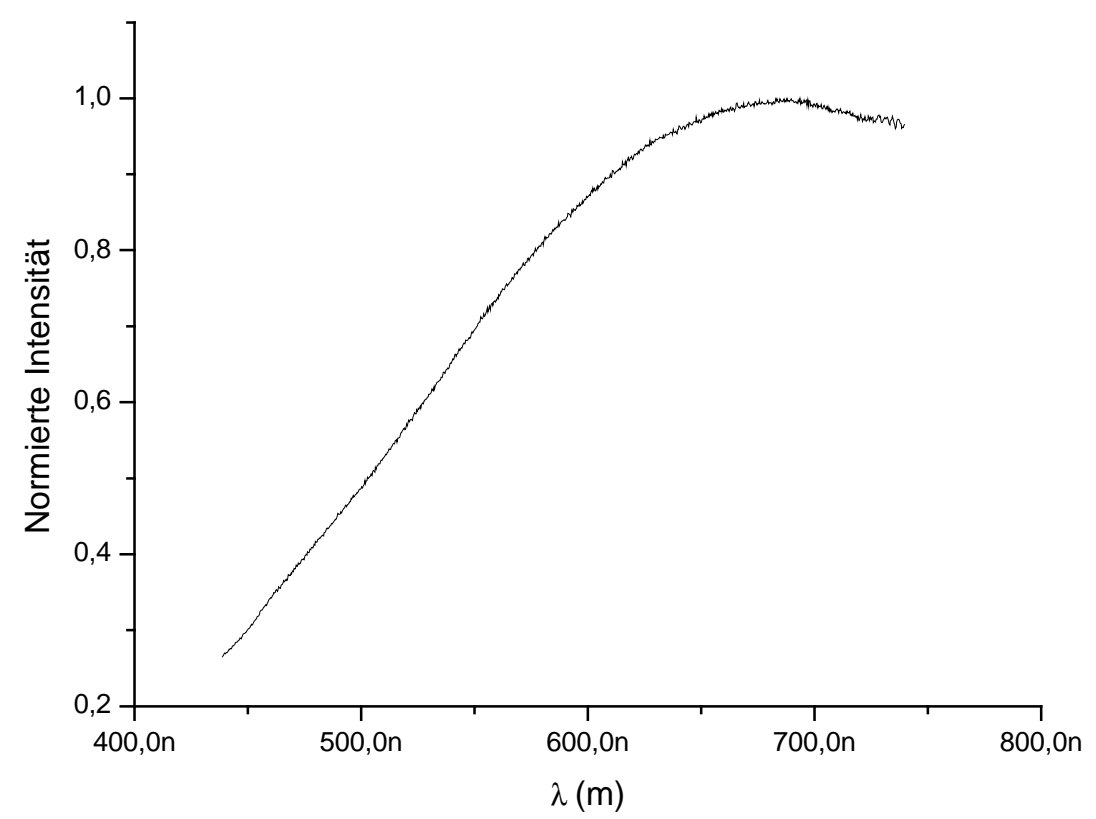

Abbildung 2.13: Gemessenes Spektrum der Halogenlampe.

Als Strahlunterbrecher diente in unserem Aufbau eine elektromagnetisch gesteuerte Aluminiumblende. Über eine Optik wird der Strahl dann, wie in Abbildung 2.12 gezeigt, auf eine Seite der Probe fokussiert. Um Einwirkungen durch Streustrahlung zu vermeiden, wird die Probe mit einer Maske bedeckt. Über die Öffnung der Maske kann auch die Breite des belichteten Bereichs genau festgelegt werden (bei den hier gemessenen Proben 1-2 mm). Die Probe selbst wird zur leichteren Handhabung zunächst auf ein schmales Stück Kunststofffolie aufgebracht, die wiederum auf eine Lochrasterplatine aufgeklebt ist. Die Manganitprobe wird dann mittels Leitsilber an den Rändern kontaktiert (siehe Abbildung 2.14). Der ganze Aufbau wird nun in einen Kryostaten mit einem Heliumkompressor (closed cycled refrigerator system, Fa. ,Air Products and Chemicals“) einmontiert, wobei der Pt100-Thermosensor die Temperatur überwacht. Dieser Sensor sitzt in einer Aussparung der Platine direkt unterhalb der Probe. Mit diesem Aufbau konnten die Proben bis zu einer Temperatur von $\mathrm{T} \approx 60 \mathrm{~K}$ abgekühlt werden. Bei der Aufnahme einer Messkurve wird zunächst das Vielkanal-Multimeter in Aufnahmebereitschaft geschaltet und dann der Strahlungsunterbrecher geöffnet. Nach der vorgeschriebenen Zeit schließt sich der Unterbrecher wieder und der Computer fängt an, die Daten Punkt für Punkt aus dem Multimeter abzulesen. Nach Ablauf einer voreingestellten Dauer wird die Messkurve im Computer gespeichert. Gleichzeitig wird das Photo-Signal von der pinDiode aufgenommen, auf die hinter dem Unterbrecher ein Teilstrahl fokussiert ist.

Während des Experimentiervorgangs wurde das System erst abgekühlt und dann langsam mit einer Rate von $1 \mathrm{~K} / \mathrm{min}$ erwärmt. Die Messung wurde nur während der langsamen Erwärmung durchgeführt, so dass alle hier präsentierten TTE-Messungen bei quasikonstanter Temperatur gemacht wurden. Danach wurde, um die Präzision der Transienten und der daraus reduzierten Parameter zu erhöhen, für jeden Temperaturbereich $\Delta \mathrm{T}=5 \mathrm{~K}$ eine Mittelung über mehrere Transienten durchgeführt. 


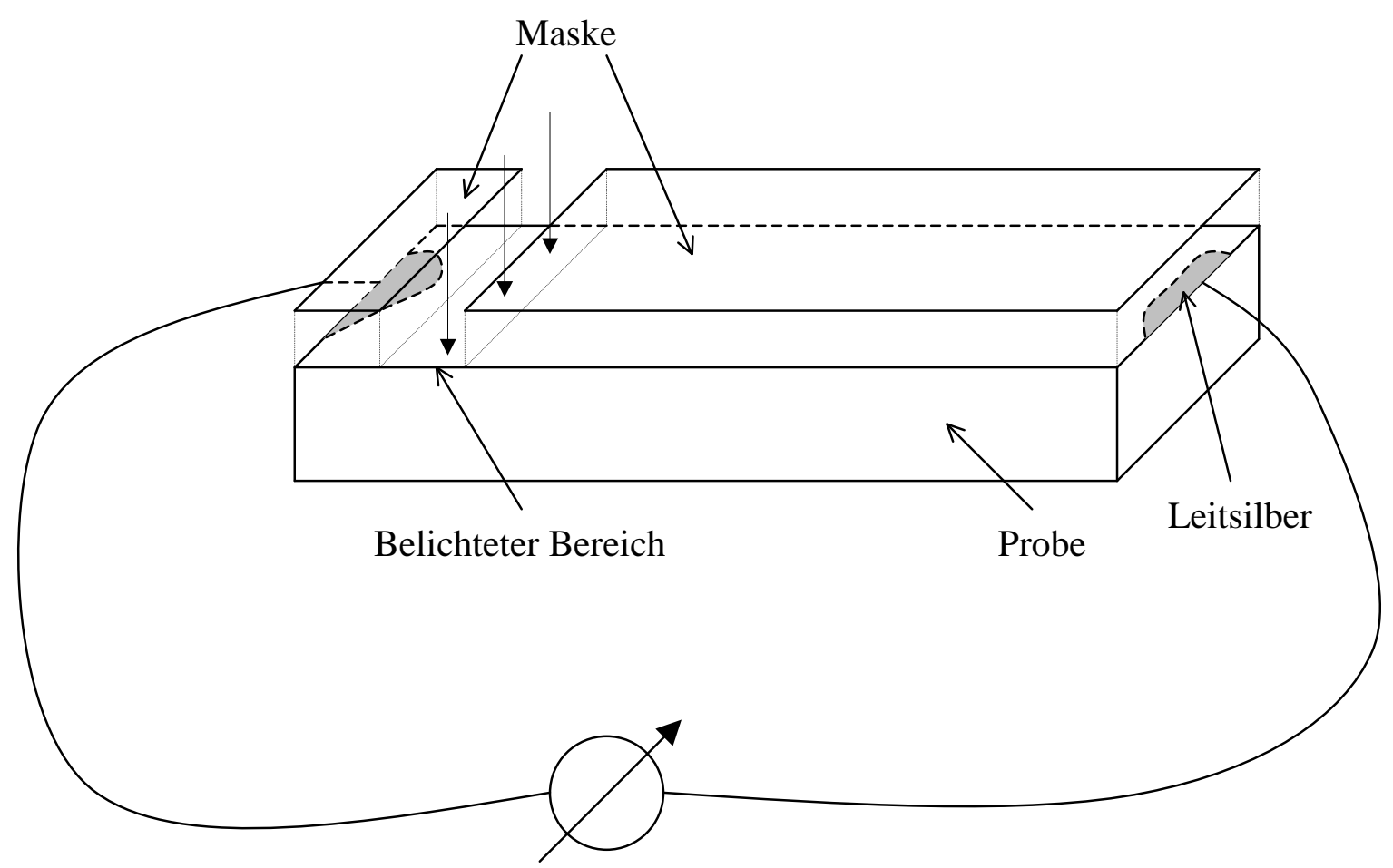

Abbildung 2.14: Die Kontaktierung der Probe und Probengeometrie.

Gibt es nur thermische Diffusion $\left(\mathrm{V}_{3}\right.$-Bereich), so wird lediglich die Anfangsamplitude $\mathrm{V}_{3}$ des dritten TTE-Transienten aufgenommen, die zu der thermoelektrischen Kraft $\mathrm{S}(\mathrm{T})$ direkt proportional ist. Aber über die absolute thermoelektrische Kraft erhält man zunächst keine Aussagen. Erstens wird bei der Messung immer einer Art thermische Kette aufgebaut, die aus den Bereichen Kupferleitung - Leitsilber - Probe - Leitsilber - Kupferleitung besteht. Empirisch zeigt sich das Problem als ein geringer linearer Beitrag $\mathrm{S}_{\mathrm{C}}$, den man von der gemessenen Thermokraft abziehen muss:

$\mathrm{S}_{\mathrm{exp}}=\mathrm{S}+\mathrm{S}_{\mathrm{C}}$

Zweitens wird die Messung bei diesem Aufbau nicht bei einem konstanten Temperaturintervall $\Delta \mathrm{T}$ durchgeführt, sondern bei einer konstanten zugeführten Wärmemenge $\Delta \mathrm{Q}$. Daher muss man zunächst $\mathrm{V}_{3}$ auf ein konstantes Temperaturintervall korrigieren. Dazu geht man folgendermaßen vor. Fällt ein konstanter Lichtimpuls auf die Probe, so empfiehlt es sich, für die Korrektur die zugeführte Wärme mit $\Delta \mathrm{Q}=\mathrm{C}_{\mathrm{p}} \Delta \mathrm{T}$ anzusetzen. Da wir die Spannung $\mathrm{V}_{3}(\mathrm{~T})$ als Funktion der Temperatur nach der Gleichung:

$\mathrm{V}_{3}(\mathrm{~T})=\mathrm{S} \Delta \mathrm{T}=\mathrm{S} \Delta \mathrm{Q} / \mathrm{C}_{\mathrm{p}}$

erhalten, steht natürlich nur das Produkt

$\mathrm{V}_{3} \mathrm{C}_{\mathrm{p}} / \mathrm{C}_{\mathrm{p}}(300 \mathrm{~K})=\mathrm{V}_{3}{ }^{*}$ 
in direkter Proportionalität $\mathrm{zu}$ unserer experimentell erhaltenen Thermokraft $\mathrm{S}_{\text {exp. Zur }}$ Kalibrierung von $\mathrm{V}_{3}{ }^{*}$ benötigt man nun noch einen Vergleich mit konventionell erhaltenen Daten, aber oft kann man die TTE-Amplituden über einen größeren Temperaturbereich messen als die konventionelle Thermokraft.

Bei der Auswertung zur Wärmeleitfähigkeit muss man folgendes berücksichtigen: die reziproke Abklingzeit ist proportional zur Wärmediffusionskonstante $\mathrm{D}$, die mit der Wärmeleitfähigkeit $\kappa$ wie folgt in Beziehung steht:

$\mathrm{D}=\kappa / \rho_{0} \mathrm{C}_{\mathrm{V}}$

wobei $\rho_{0}$ die Dichte und $C_{V}$ die spezifische Wärmekapazität ist. Im Festkörper kann man zudem mit großer Genauigkeit $C_{V}=C_{p}$ setzen, sodass eine zusätzliche Messung von $C_{p}(T)$ zur absoluten Bestimmung von $\mathrm{K}$ genügt. 


\section{Messergebnisse}

\subsection{Temperatur- und Magnetfeldabhängigkeit der Magnetisierung}

Da die magnetischen Eigenschaften in Manganiten normalerweise sehr stark mit den Transporteigenschaften verbunden sind, haben wir zuerst versucht festzustellen, welche magnetischen Zustände innerhalb des Probenvolumens und bei verschiedenen Temperaturen auftreten. Dafür wurden das magnetische Moment $\mathrm{M}(\mathrm{T})$ und die reziproke Suszeptibilität $\chi^{-1}(\mathrm{~T})$ von den keramischen $\mathrm{Nd}_{2 / 3} \mathrm{Sr}_{1 / 3} \mathrm{MnO}_{3-\delta}$-Proben aus der Serie $1(\delta=0,0.05,0.1,0.15,0.2)$ von Stickstofftemperatur bis zu $350 \mathrm{~K}$ gemessen. Für die zweite Serie $(\delta=0,0.02,0.07,0.12,0.17)$ wurden die Messungen M(T) mit und ohne Magnetfeld (zerofield cooled „ZFC“ und field cooled „FC“) im Bereich von Heliumtemperatur bis Raumtemperatur durchgeführt. Für die zweite Serie wurden auch $\mathrm{M}(\mathrm{H})$-Messungen durchgeführt.

Die Magnetisierungsmessungen $\mathrm{M}(\mathrm{T})$ der zweiten Probenserie wurden in einem Feld von $\mathrm{H}=$ 100 Oe und von Heliumtemperatur bis Raumtemperatur mit Hilfe eines Vibrationsmagnetometer (QI-3001) durchgeführt ${ }^{6}$. Diese Messungen sind in der Abbildung 3.1 zusammengefasst. Man sieht einen deutlichen Unterschied zwischen den ZFC- und FC-Kurven bei den Proben mit größeren $\delta$, was insbesondere bei kleinen Magnetisierungen $M$ auf eine erinnerungsabhängige antiferromagnetische Komponente hinweist.

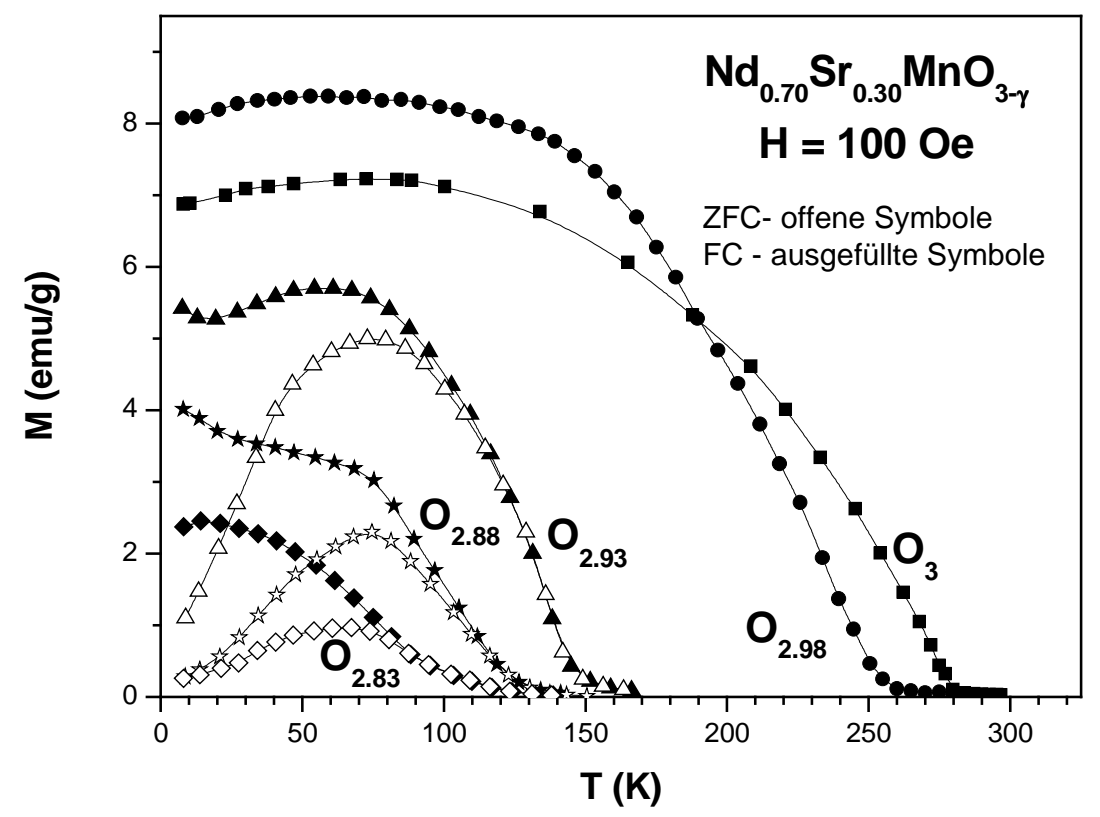

Abbildung 3.1: Temperaturabhängigkeit der Magnetisierung $M(T)$, gemessen an der zweiten Serie von den $\mathrm{Nd}_{2 / 3} \mathrm{Sr}_{1 / 3} \mathrm{MnO}_{3-\delta}$-Proben. Gefüllte Symbole: Abkühlung im H-Feld (FC), nicht gefüllte Symbole: Abkühlung ohne magnetisches Feld (ZFC).

\footnotetext{
${ }^{6}$ Für die magnetischen Messungen an der zweiten Probenserie sind wir Prof. H. Szymczak (Institute of Physics, Polish Academy of Sciences) und Prof. I. Troyanchuk (Institute of Physics of Solid State and Semiconductor, NAS of Belarus) dankbar.
} 
Auch die $\mathrm{M}(\mathrm{H})$-Kurven deuten auf abnehmende magnetische Gesamtmomente, d.h. eine Zunahme der Spinkompensation mit zunehmendem O-Defizit (siehe Abbildung 3.2).

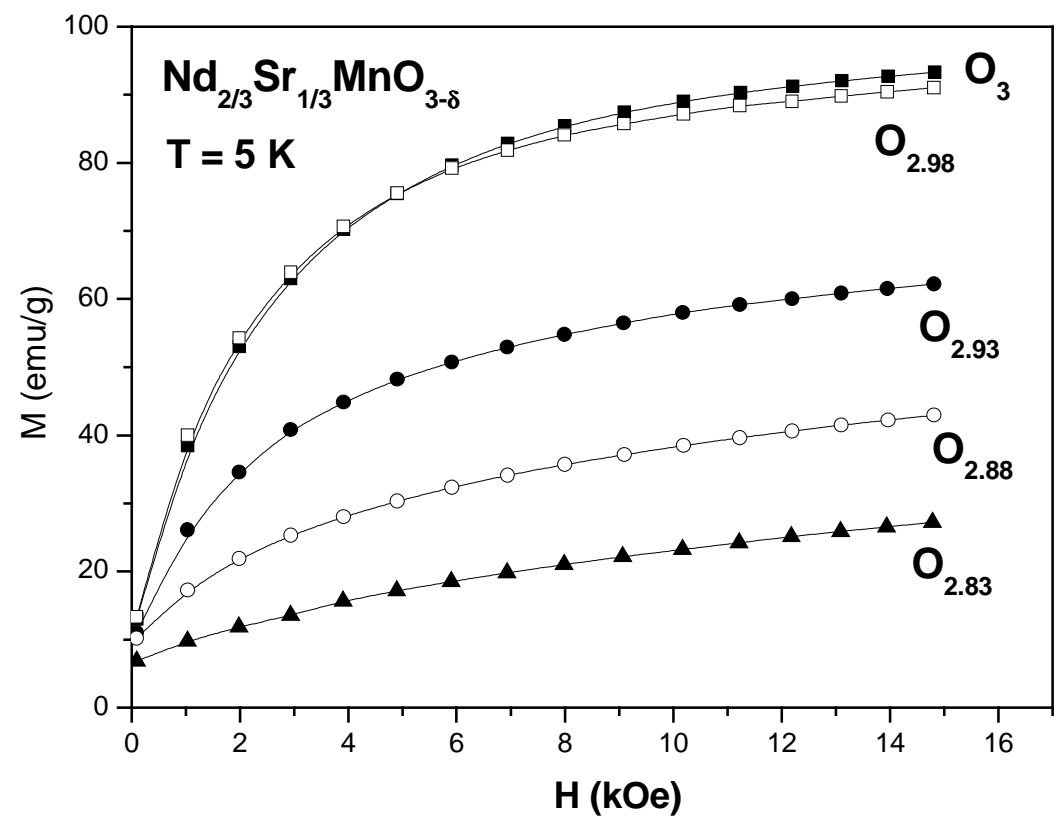

Abbildung 3.2: Die M(H)-Kurven für die Proben aus der Serie 2 bei 5 K.

Da die oben präsentierten magnetischen Messungen überwiegend im Bereich der spontanen Magnetisierung durchgeführt worden sind, haben wir bei den Messungen an der Serie 1 dem paramagnetischen Bereich besondere Aufmerksamkeit gewidmet.

Bei den magnetischen Messungen an der ersten Probenserie (1) wurde die Faraday-Methode verwendet. Alle Proben wurden in einem Messfeld von $\mathrm{H}=0.25 \mathrm{kOe}$ untersucht. Die $\mathrm{M}(\mathrm{T})-$ Messung für die stoichiometrische Verbindung $\mathrm{Nd}_{2 / 3} \mathrm{Sr}_{1 / 3} \mathrm{MnO}_{3}$ (Abb. 3.3) stimmt mit den Literaturdaten [11] gut überein. Paramagnetische Curie-Temperatur, ferromagnetische CurieTemperatur und die Metall-Halbleiter-Übergangstemperatur sind in diesem Fall gleich $\left(\theta_{\mathrm{f}}=\mathrm{T}_{\mathrm{C}}=\right.$ $\left.\mathrm{T}_{\mathrm{mi}}=248 \mathrm{~K}\right)$. Die paramagnetische Curie-Temperatur $\theta_{\mathrm{f}}$ wurde durch die Anpassung des Hochtemperatur-Bereiches der reziproken Suszeptibilität $\chi^{-1}(\mathrm{~T})$ mit Hilfe des Curie-Weiss'schen Gesetzes bestimmt. Für die ferromagnetische Curie-Temperatur $T_{C}$ wurde der Punkt der maximalen Steigung in der $\mathrm{M}(\mathrm{T})$-Kurve genommen $\left(\mathrm{d}^{2} \mathrm{M}(\mathrm{T}) / \mathrm{dT}^{2}=0\right)$. Es gibt aber noch weitere Methoden zur Bestimmung von $\mathrm{T}_{\mathrm{C}}$. Zum Beispiel kann man die Tangente am Punkt der maximalen Steigung einzeichnen und dann als $\mathrm{T}_{\mathrm{C}}$-Wert den Kreuzungspunkt dieser Tangente mit der T-Achse nehmen. Die nach dieser Methode bestimmte ferromagnetische Curie-Temperatur $\mathrm{T}_{\mathrm{C}}$ ' beträgt $257 \mathrm{~K}$. Dieser Wert ist um $9 \mathrm{~K}$ größer.

Während die paramagnetische Curie-Temperatur $\theta_{\mathrm{f}}$ der Probe mit dem Sauerstoffdefizit $\delta=0.05$ bei $147 \mathrm{~K}$ liegt, findet man die ferromagnetische Curie-Temperatur $\mathrm{T}_{\mathrm{C}}$ (ebenfalls bestimmt aus dem Wendepunkt: $\mathrm{d}^{2} \mathrm{M} / \mathrm{dT}^{2}=0$ ) bei $134 \mathrm{~K}$. Allerdings ist der Anstieg der Magnetisierung bereits wesentlich flacher. Der Wert von T' ${ }_{\mathrm{C}}=162$ (bestimmt mit Hilfe der Tangente am Punkt der 
maximalen Steigung) ist wiederum größer. Die Temperaturabhängigkeit der Magnetisierung und die reziproke Suszeptibilität dieser Probe sind in der Abbildung 3.4 gezeigt.

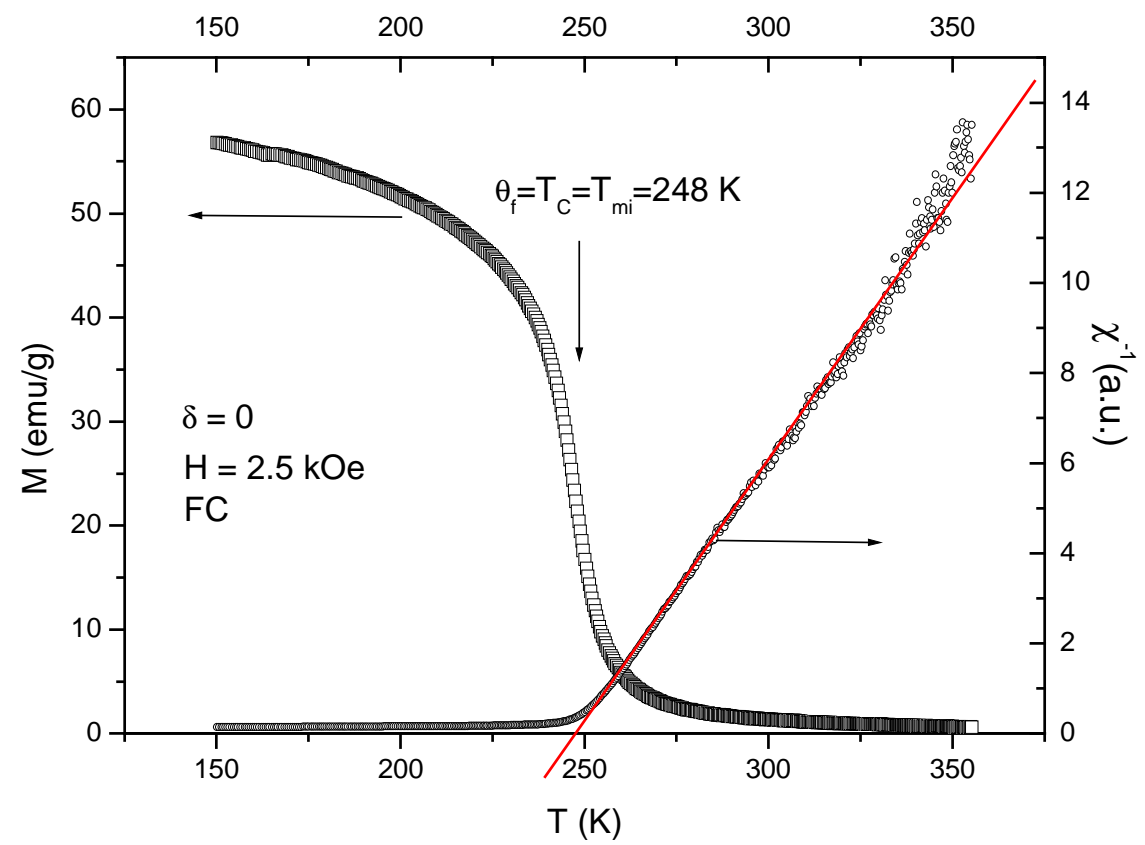

Abbildung 3.3: Temperaturabhängigkeit der Magnetisierung $\mathrm{M}(\mathrm{T})$ einer $\mathrm{Nd}_{2 / 3} \mathrm{Sr}_{1 / 3} \mathrm{MnO}_{3}$-Probe und die reziproke Suszeptibilität $\chi^{-1}(T)$.

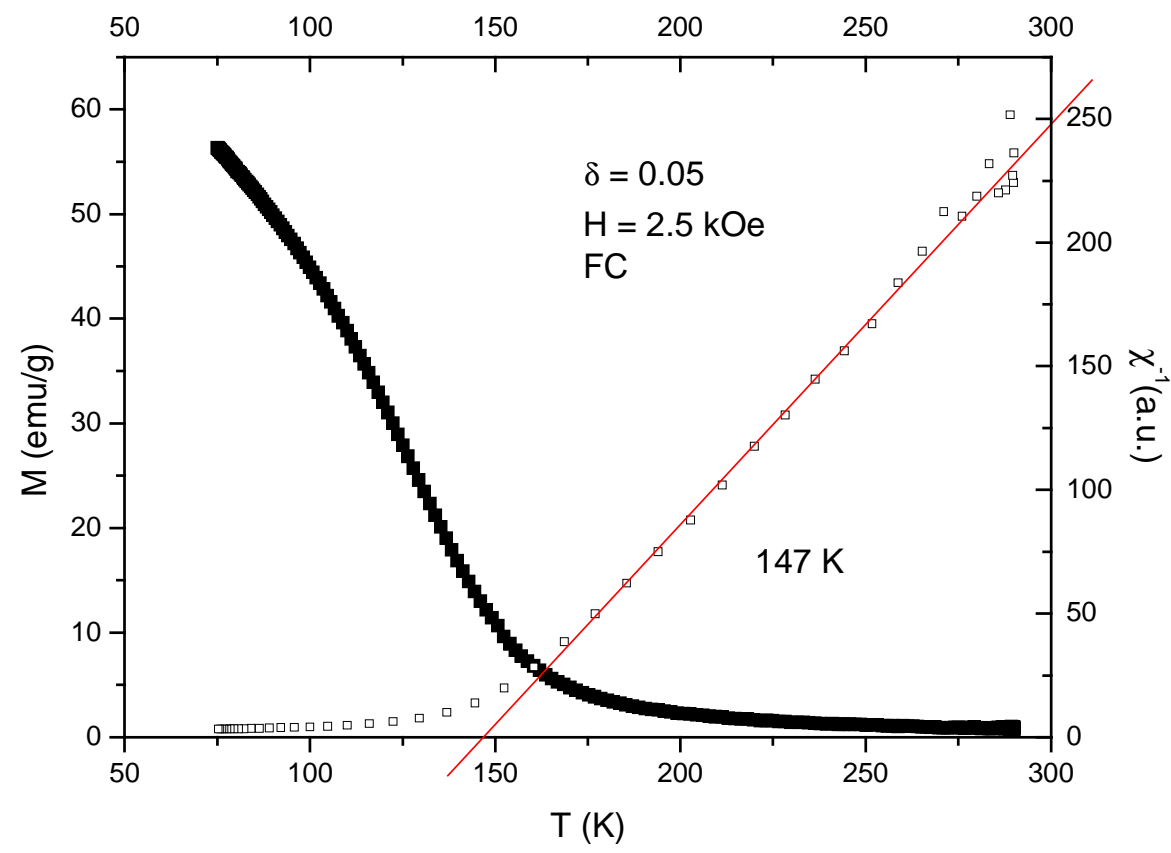

Abbildung 3.4: Temperaturabhängigkeit der Magnetisierung $\mathrm{M}(\mathrm{T})$ einer $\mathrm{Nd}_{2 / 3} \mathrm{Sr}_{1 / 3} \mathrm{MnO}_{2.95^{-}}$ Probe und die reziproke Suszeptibilität $\chi^{-1}(T)$.

Die Situation bei der $\mathrm{Nd}_{2 / 3} \mathrm{Sr}_{1 / 3} \mathrm{MnO}_{3-\delta}$-Probe mit $\delta=0.10$ ist schon deutlich komplizierter. Bei der Anpassung des linkes Teiles der reziproken Suszeptibilität $\chi^{-1}(\mathrm{~T})$ (siehe in der Abbildung 3.5 die Fit-Kurve 1) nach dem Curie-Weiß'schen Gesetz ergibt sich die paramagnetische Curie- 
Temperatur zu $\theta_{\mathrm{f}}=90 \mathrm{~K}$. Bei höheren Temperaturen findet man allerdings einen Bereich (Fit 2), in dem die $\chi^{-1}(\mathrm{~T})$-Kurve mit einem zweiten Curie-Weiß'schen Gesetz angepasst werden kann. Aus dieser Extrapolation bekommt man die Curie-Weiß-Temperatur $\theta_{\mathrm{a}} \approx-200 \mathrm{~K}$, was wiederum auf eine antiferromagnetische Komponente in der Probe hinweist.

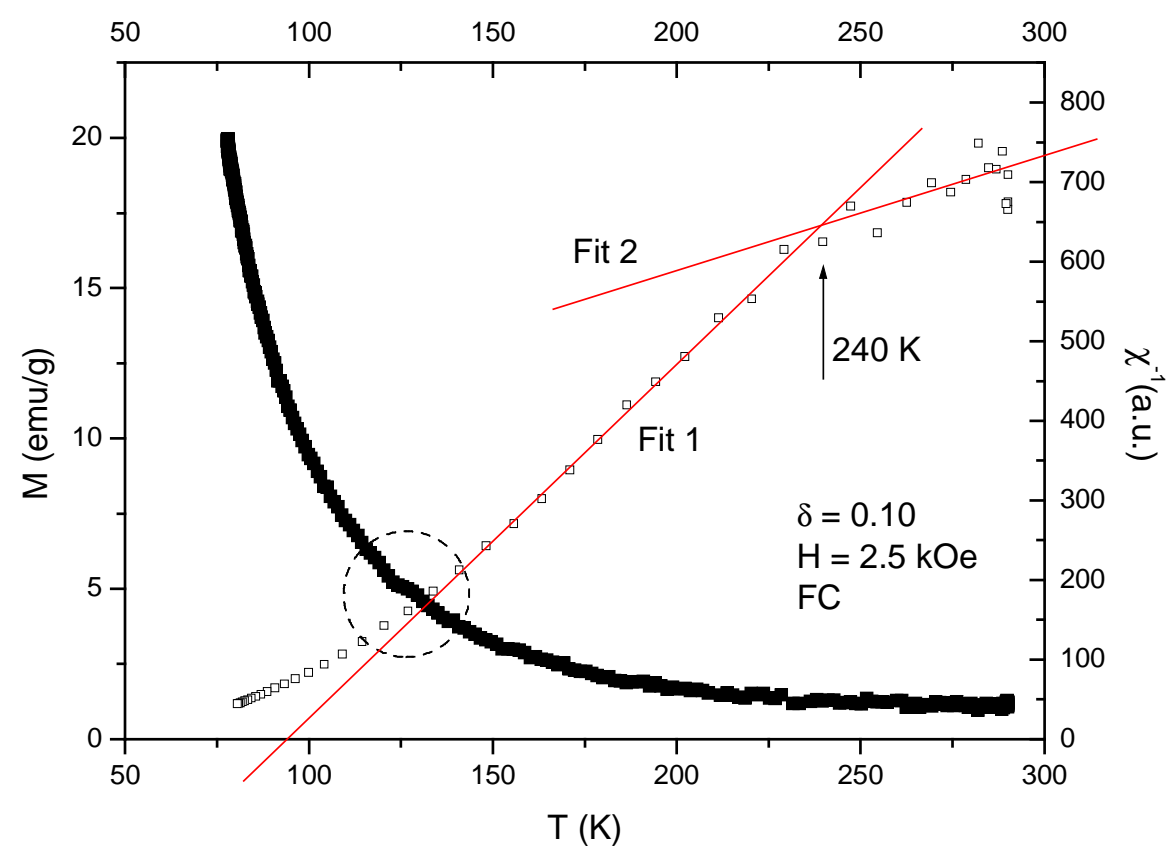

Abbildung 3.5: Temperaturabhängigkeit der Magnetisierung $\mathrm{M}(\mathrm{T})$ einer $\mathrm{Nd}_{2 / 3} \mathrm{Sr}_{1 / 3} \mathrm{MnO}_{2.90^{-}}$ Probe und die reziproke Suszeptibilität $\chi^{-1}(T)$.

In der Nähe von $125 \mathrm{~K}$ kann man zudem eine schwache Struktur in der M(T)-Kurve vermuten. Hier könnte es also einen Übergang in einen etwas anderen magnetischen Zustand geben. Allerdings benötigt man zur Sicherung dieses Ergebnisses noch eine genauere oder eine unabhängige Messung (siehe unten). Zunächst wollen wir aber die Serie abschließen.

Für die Probe mit $\delta=0.15$ beobachten wir ebenfalls zwei verschiedenen Bereiche in dem $\chi^{-1}(\mathrm{~T})$ Verlauf (sieh Abbildung 3.6). Einer davon ergibt eine paramagnetische Temperatur $\theta_{\mathrm{f}}=50 \mathrm{~K}$, der zweite führt uns wieder in den negativen Temperaturbereich.

Vermutlich gibt es wieder eine kleine Anomalie. Die Wiederholung der Messung mit einer kleineren Stoffmenge (der Probe mit $\delta=0.15$ ) zeigt in der Tat eine deutlichere Anomalie, aber in der Nähe von $150 \mathrm{~K}$. Diese M(T)-Kurve wird in der Abbildung 3.7 präsentiert. Diese Anomalie ist in der Tat auch ähnlich der schwachen Struktur, die bei der Probe mit $\delta=0.10$ gefunden wurde. Die bessere Auflösung von M bei Abbildung 3.7 liegt vermutlich daran, dass bei einer Verringerung der Probenmenge die Verteilung des O-Defizits schmaler wird. Hier zeigt sich auch bei $250 \mathrm{~K}$ eine zweite Anomalie, die der bei $240 \mathrm{~K}(\delta=0.10)$ in $\chi^{-1}$ beobachteten Anomalie ähnlich ist. 


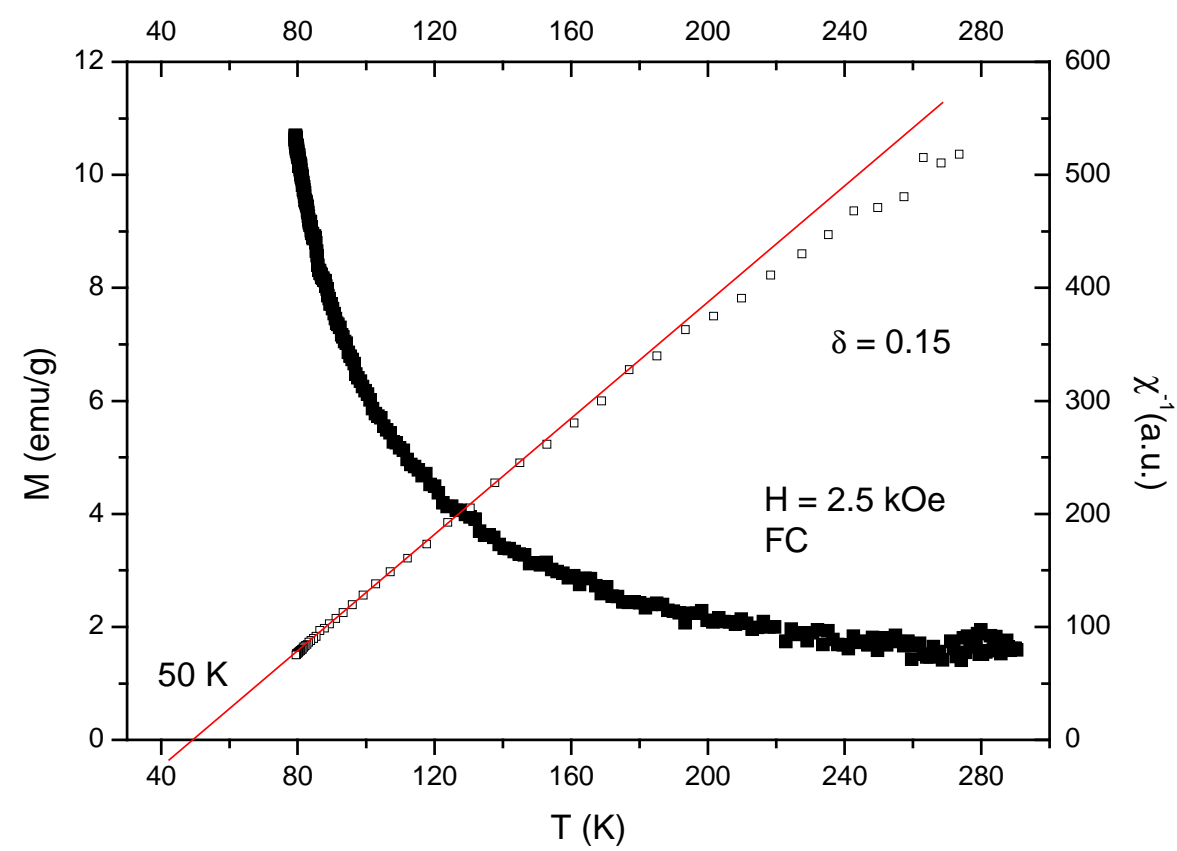

Abbildung 3.6: Temperaturabhängigkeit der Magnetisierung $\mathrm{M}(\mathrm{T})$ einer $\mathrm{Nd}_{2 / 3} \mathrm{Sr}_{1 / 3} \mathrm{MnO}_{2.85^{-}}$ Probe und die reziproke Suszeptibilität $\chi^{-1}(T)$.

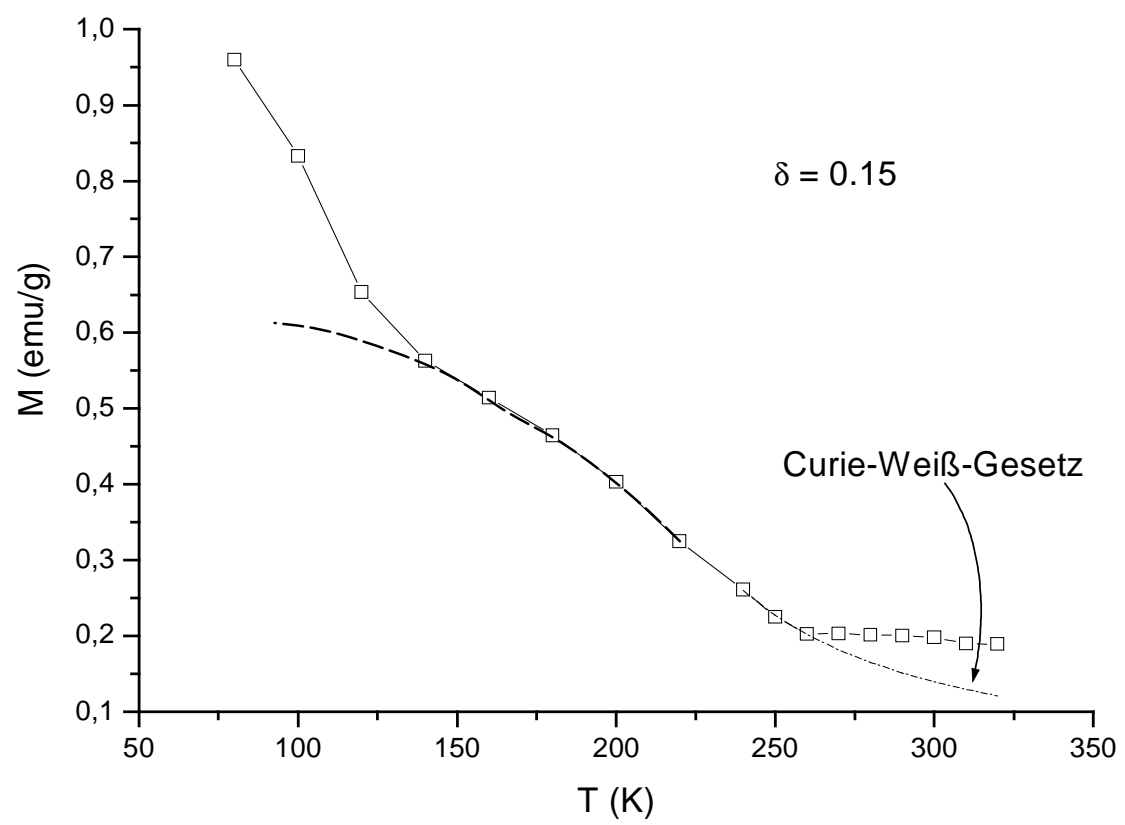

Abbildung 3.7: Temperaturabhängigkeit der Magnetisierung $M(T)$ einer $\mathrm{Nd}_{2 / 3} \mathrm{Sr}_{1 / 3} \mathrm{MnO}_{2.85^{-}}$ Probe mit der Masse 12.72 mg, gemessen in einem Feld H = 100 Oe.

Die Anomalie in $\mathrm{M}(\mathrm{T})$ bei $125 \mathrm{~K}(\delta=0.15)$ ist schwach und könnte daher in Zweifel gezogen werden. Allerdings zeigen auch die Messungen der Magnetisierung $\mathrm{M}(\mathrm{H})$ unter der konstanten Temperatur im Bereich von 0 bis $12 \mathrm{kOe}$, dass die $\mathrm{M}(\mathrm{H})$-Kurven oberhalb der Temperatur $150 \mathrm{~K}$ linear sind, aber unterhalb dieser Temperatur deutlich nichtlinear. Dies bestätigt, dass unterhalb 
$125 \mathrm{~K}$ ein leicht veränderter stabiler magnetischer Zustand in der Probe auftritt (siehe Abbildung $3.8)$.

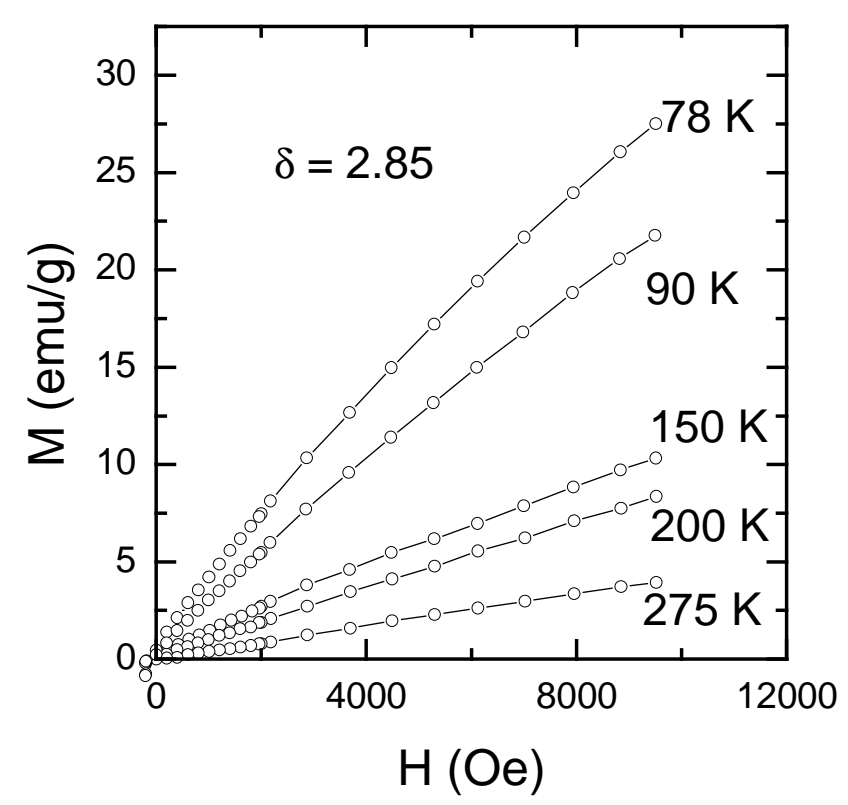

Abbildung 3.8: $M(H)$-Messungen an der Probe mit $\delta=0.15$ unter verschiedenen Temperaturen im Bereich von $78 \mathrm{~K}$ bis $275 \mathrm{~K}^{7}$.

Die Messung an der Probe mit dem größten Sauerstoffdefizit $\delta=0.20$ wird in der Abbildung 3.9 dargestellt. Aus der Extrapolation der linearen Anpassung entnehmen wir für eine paramagnetische Curie-Temperatur $\theta_{\mathrm{f}}=24 \mathrm{~K}$. Nachweisbare Anomalien finden wir hier nicht.

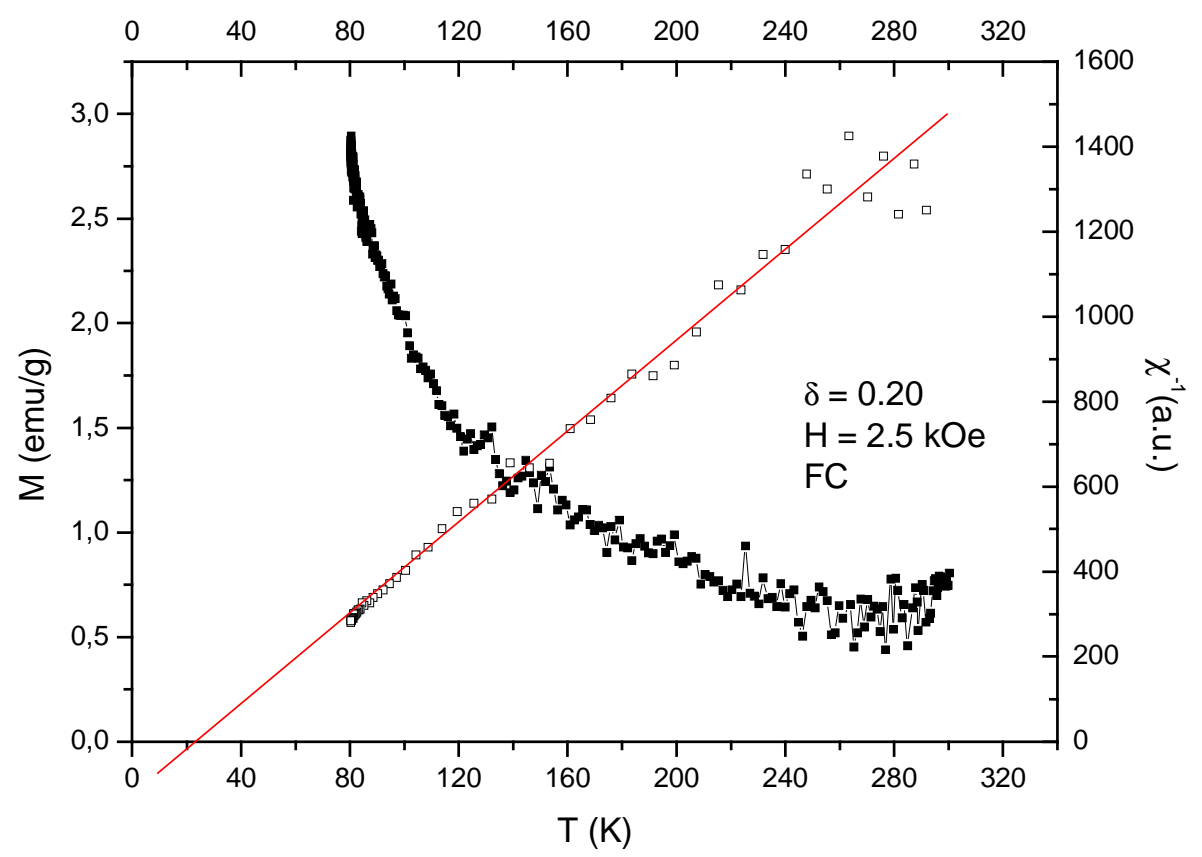

Abbildung 3.9: Temperaturabhängigkeit der Magnetisierung $\mathrm{M}(\mathrm{T})$ einer $\mathrm{Nd}_{2 / 3} \mathrm{Sr}_{1 / 3} \mathrm{MnO}_{2.80^{-}}$ Probe und die reziproke Suszeptibilität $\chi^{-1}(T)$.

\footnotetext{
${ }^{7}$ Für diese Messungen danken wir Dr. C. P. Yang (Tsinghua-Universität, Peking, China), der im Jahr 2002 als Humboldt-Stipendiat in unserer Arbeitsgruppe zu Gast war.
} 


\subsection{Das $T_{C}-\delta$ Diagramm}

In der Abbildung 3.10 wurden die charakteristischen magnetischen Temperaturen (ferromagnetische und paramagnetische Curie-Temperatur) gegen das Sauerstoffdefizit $\delta$ aufgetragen. Die Sternsymbole mit einem Apostroph sind die ferromagnetischen CurieTemperaturen $\mathrm{T}_{\mathrm{C}}$, , die mit Hilfe der Tangente bestimmt wurden. Aus dem Diagramm sieht man, dass bei dem größeren Sauerstoffdefizit $(\delta>0.07)$ die paramagnetischen und die ferromagnetischen Curie-Temperaturen zwei unterschiedlichen Zweige bilden. Dabei liegen die paramagnetischen Temperaturen $\theta_{\mathrm{f}}$ deutlich tiefer als die ferromagnetischen $\mathrm{T}_{\mathrm{C}}$-Temperaturen. Wir haben schon bei den Gitterparametern auf eine Anomalie bei der $\delta$-Variation (bei $\delta \approx 0.078$ ) hingewiesen (siehe Kapitel „Experimentelles“) und dabei ein zweites $\delta$-Regime vermutet. Die magnetischen Messungen liefern damit noch einen weiteren Beweis, dass sich bei $\delta>0.07$ ein zweites Regime ausbildet.

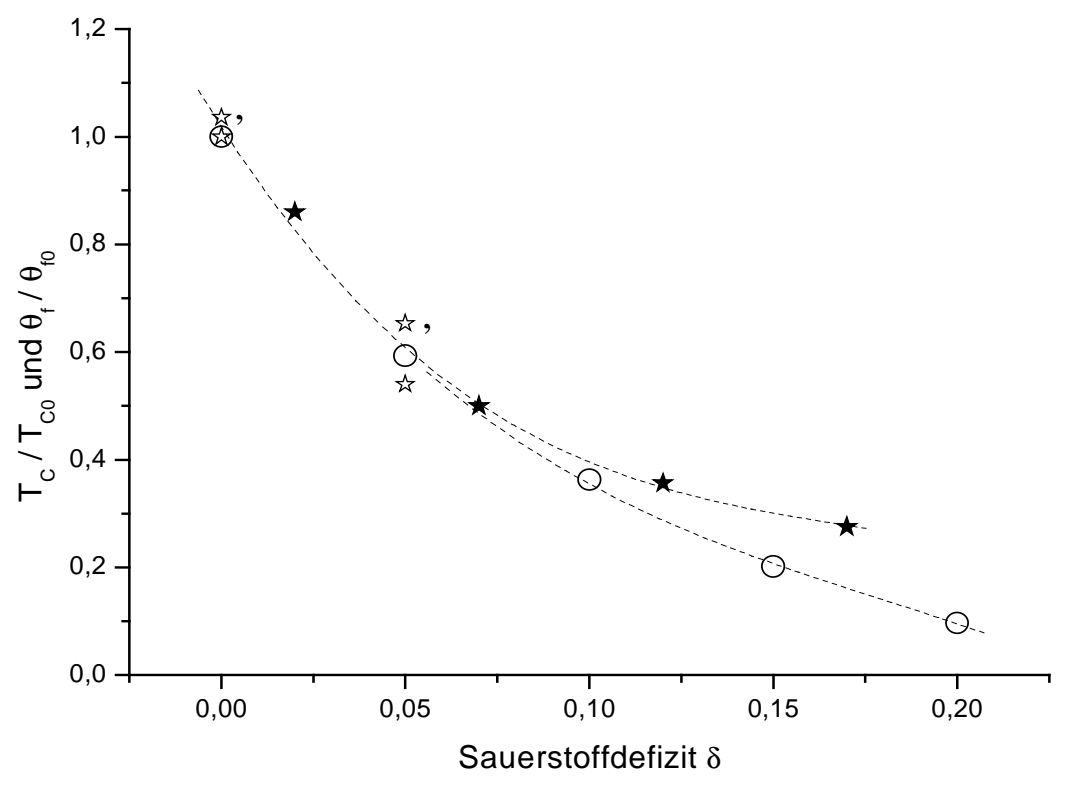

Abbildung 3.10: Die $T_{C} / T_{C 0^{-}} \delta$ (Sternsymbole) und $\theta_{f} / \theta_{f 0^{-}} \delta$ (runde Symbole) Phasendiagramm. $T_{C 0}$ und $\theta_{f 0}$ sind ferromagnetische und paramagnetische Curie-Temperatur bei $\delta=0$. Gefüllte Symbole gehören zur Serie 2, nicht gefüllte Symbole gehören zur Serie 1.

In der Tabelle 3.1 wurden alle charakteristischen Temperaturen, die aus den magnetischen Messungen erworben sind, zur besseren Übersicht zusammengefasst.

Serie 1

\begin{tabular}{|c|c|c|c|}
\hline$\delta$ & $\theta_{\mathrm{f}}(\mathrm{K})$ & $\mathrm{T}_{\mathrm{C}}(\mathrm{K})$ & $\left.\mathrm{T}^{{ }_{\mathrm{C}}}{ }_{\mathrm{K}}\right)$ \\
\hline 0.00 & 248 & 248 & 257 \\
\hline 0.05 & 147 & 134 & 162 \\
\hline 0.10 & 90 & - & - \\
\hline 0.15 & 50 & - & - \\
\hline 0.20 & 24 & - & - \\
\hline
\end{tabular}


Serie 2

\begin{tabular}{|c|c|c|c|}
\hline$\delta$ & $\theta_{\mathrm{f}}(\mathrm{K})$ & $\mathrm{T}_{\mathrm{C}}(\mathrm{K})$ & $\mathrm{T}^{{ }_{\mathrm{C}}}(\mathrm{K})$ \\
\hline 0.00 & - & 272 & 281 \\
\hline 0.02 & - & 234 & 255 \\
\hline 0.07 & - & 136 & 148 \\
\hline 0.12 & - & 97 & 125 \\
\hline 0.17 & - & 75 & 103 \\
\hline
\end{tabular}

Tabelle 3.1: Die charakteristischen magnetischen Temperaturen für die zwei Serien von keramischen $\mathrm{Nd}_{2 / 3} \mathrm{Sr}_{1 / 3} \mathrm{MnO}_{3-\delta}$-Proben. $\theta_{f}$ - die paramagnetische Curie-Temperatur, $T_{C}-$ die ferromagnetische Curie-Temperatur (bestimmt als der Punkt der maximalen Steigung der M(T)Kurve), $T{ }_{C}{ }^{-}$-die ferromagnetische Curie-Temperatur (bestimmt als der Punkt der Kreuzung der Tangente am Punkt der maximalen Steigung mit der T-Achse).

3.3. Spezifischer Widerstand und V(I)-Charakteristiken der keramischen $\mathrm{Nd}_{2 / 3} \mathrm{Sr}_{1 / 3} \mathrm{MnO}_{3}$ $\delta$-Proben

In der Abbildung 3.4 ist der an der stoichiometrischen Verbindung $\mathrm{Nd}_{2 / 3} \mathrm{Sr}_{1 / 3} \mathrm{MnO}_{3}$ gemessene spezifische Widerstand $\rho$ aufgetragen.

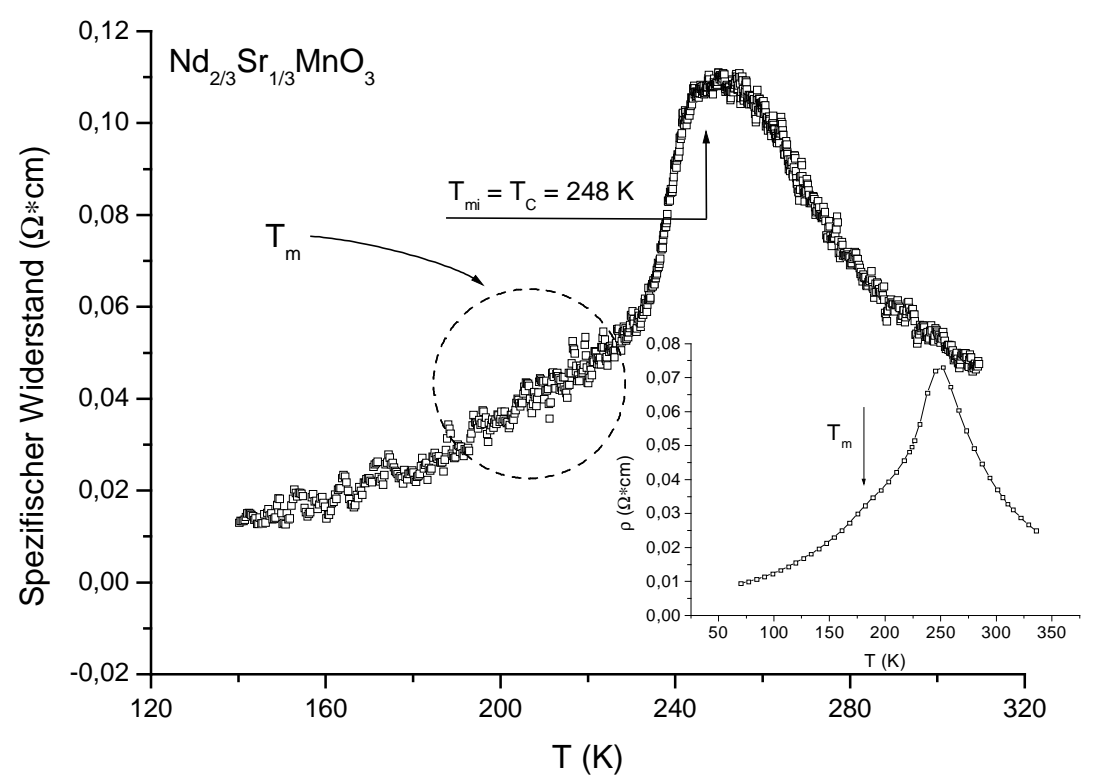

Abbildung 3.4: Spezifischer Widerstand der keramischen $\mathrm{Nd}_{2 / 3} \mathrm{Sr}_{1 / 3} \mathrm{MnO}_{3}$-Probe. Inset: Spezifischer Widerstand einer $\mathrm{Nd}_{2 / 3} \mathrm{Sr}_{1 / 3} \mathrm{MnO}_{3-\delta}$ Keramik mit kleinem $\delta$ nach [11]. Eine Schulter ( $T_{m}$-Maximum) ist deutlich zu erkennen.

Die Messung zeigt, dass die Probe einen Metall-Isolator-Übergang in der Nähe von $248 \mathrm{~K}$ hat. Aus dem Vergleich mit den Magnetisierungsmessungen sieht man, dass die Übergangstemperatur $\mathrm{T}_{\mathrm{mi}}$ mit der ferromagnetischen Curie-Temperatur $\mathrm{T}_{\mathrm{C}}$ übereinstimmt, was allgemein eine starke Kopplung der magnetischen und elektrischen Eigenschaften nahelegt. Das 
hier beobachtete Maximum im $\rho(\mathrm{T})$-Verlauf wird in der Literatur mit einer Volumeneigenschaft verbunden (intrinsischer Effekt). Das normalerweise bei keramischen Proben erwartete zusätzliche zweite Maximum unterhalb $\mathrm{T}_{\mathrm{mi}}$ ist in dieser $\mathrm{R}(\mathrm{T})$-Kurve aber kaum zu sehen (Schulter unterhalb $\mathrm{T}_{\mathrm{mi}}$ ). Das zweite Maximum bei $\mathrm{T}_{\mathrm{m}}$ wird oft beobachtet und durch eine spinabhängige Streuung an den Korngrenzen erklärt [6, 13] (extrinsischer Effekt).

Die V(I)-Charakteristiken bei verschiedenen Temperaturen sind in Abbildung 3.12 zu finden. Wie man sieht, sind alle Kennlinien im gemessenen Temperaturbereich linear. Das bedeutet, dass diese Probe oberhalb und unterhalb des Curie-Punktes ein normales Ohm'sches Verhalten zeigt, d.h. dass die Leitfähigkeit unabhängig von dem durch die Probe fließenden Strom ist. Die gemessenen Kennlinien sind auch im ganzen Temperaturbereich reversibel, wie erwartet.

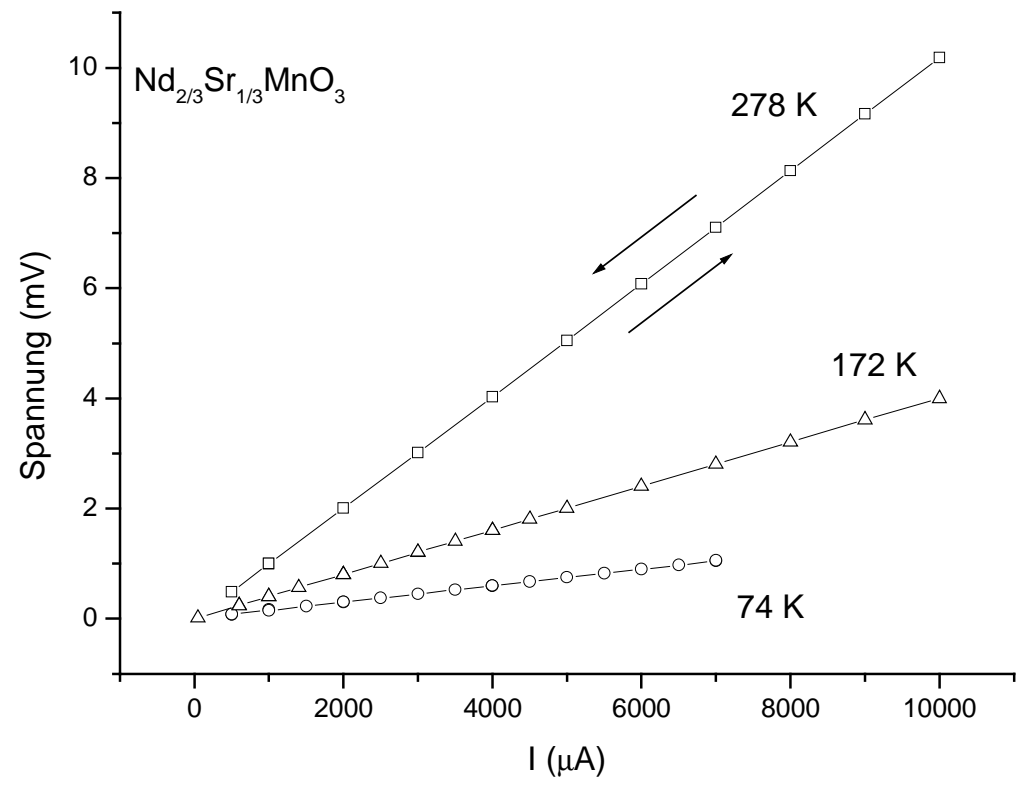

Abbildung 3.12: Die an der $\mathrm{Nd}_{2 / 3} \mathrm{Sr}_{1 / 3} \mathrm{MnO}_{3}$-Probe gemessenen Kennlinien zeigen Ohm'sches Verhalten.

Die Probe mit dem Sauerstoffdefizit $\delta=0.05$ (aus der ersten Serie) zeigt bereits ein komplexes Widerstandsverhalten. Bei dieser Probe hängt der Widerstand überraschenderweise stark vom eingeprägten Strom ab. Je größer der Strom, der bei der Messung benutzt wird, desto höher wird die Leitfähigkeit der Probe. Da die Stromabhängigkeit des Widerstandes R einer Abhängigkeit von $\mathrm{R}$ vom elektrischen Feld zwischen oder über die Korngrenzen äquivalent ist, könnte man diesen Effekt auch als einen elektroresistiven Effekt oder „Elektrowiderstand“ bezeichnen [16]. Wie aus der Abbildung $3.13 \mathrm{zu}$ entnehmen, ist die Probe bei Raumtemperatur zwar noch Ohm'sch, aber unterhalb einer Temperatur $\mathrm{T}_{\mathrm{B}} \approx 230 \mathrm{~K}$ tritt ein stark nichtlineares Verhalten in $\mathrm{V}(\mathrm{I})$ auf, das aber bei einer Temperatur $\approx 55 \mathrm{~K}$ praktisch wieder verschwindet. Man hat also hier einen nichtlinearen Bereich, der durch zwei Verzweigungspunkte $T_{B}$ und $T_{B}$ ' definiert ist. 


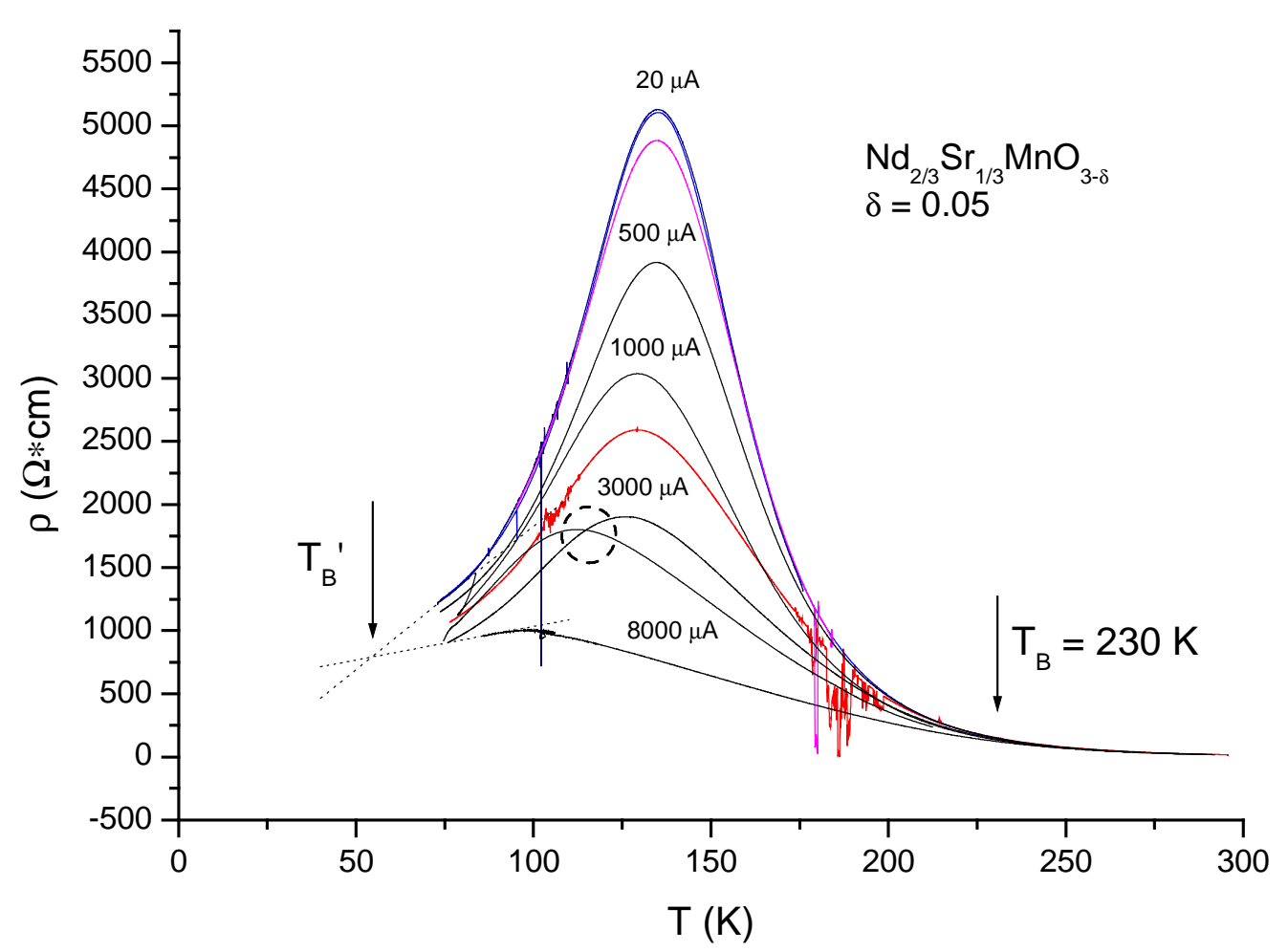

Abbildung 3.13: Spezifischer Widerstand der keramischen $\mathrm{Nd}_{2 / 3} \mathrm{Sr}_{1 / 3} \mathrm{MnO}_{2.95}$-Probe, gemessen bei verschiedenen Strombelastungen. Der gestrichelte Kreis zeigt den Übergang vom positiven zum negativen differentiellen ER-Effekt. Die Schwankungen (rot) sind Instabilitäten (siehe Anhang A).

In der Abbildung 3.14 sind die bei verschiedenen Temperaturen aufgenommenen Kennlinien V(I) aufgetragen.

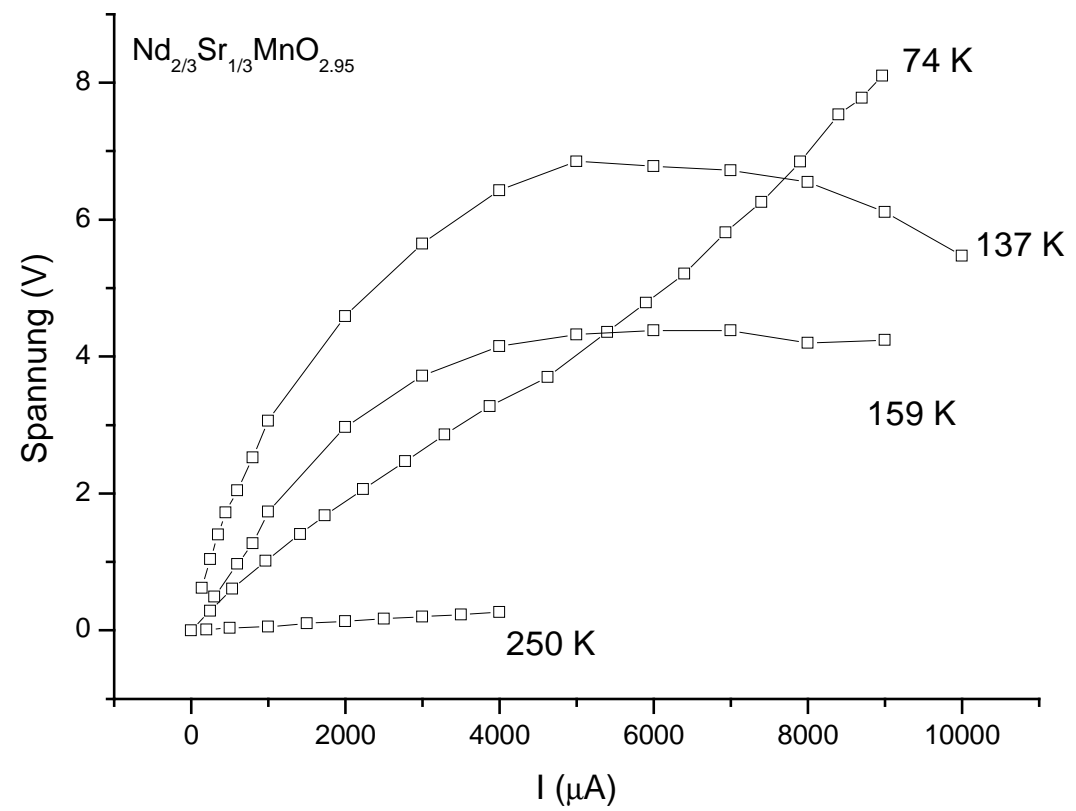

Abbildung 3.14: Die an der $\mathrm{Nd}_{2 / 3} \mathrm{Sr}_{1 / 3} \mathrm{MnO}_{2.95}$-Probe gemessenen Kennlinien zeigen bei den tieferen Temperaturen ein stark nichtlineares Verhalten. 
Man sieht hier wieder, dass die V(I)-Charakteristik im Bereich der höheren Temperaturen $T>T_{B}$ (hier nur für $\mathrm{T}=250 \mathrm{~K}$ ) linear ist. Bei den tieferen Temperaturen (159 und $137 \mathrm{~K}$ ) sehen wir dagegen eine starke Krümmung der V(I)-Kurven. Bei noch tieferen Temperaturen (hier $\leq 74 \mathrm{~K}$ ) werden die Kennlinien tendenziell wieder linear. Dadurch definiert sich ein mittlerer Temperaturbereich für den ER-Effekt, $\mathrm{T}_{\mathrm{B}}{ }_{\mathrm{B}}<\mathrm{T}<\mathrm{T}_{\mathrm{B}}$.

Wenn wir die mit dem kleinsten Strom gemessene $\rho(\mathrm{T})$-Kurve betrachten, liegt das Maximum von $\rho(\mathrm{T})$ bei einer Temperatur $\mathrm{T}_{\mathrm{m}}=134 \mathrm{~K}$. Dieses Maximum ist vermutlich (im Gegenteil der Probe mit $\delta=0$ ) schon ein sekundäres Maximum, das man dem Transport durch Korngrenzen zuordnet. Für die sauerstoffdefizitären $\mathrm{Nd}_{2 / 3} \mathrm{Sr}_{1 / 3} \mathrm{MnO}_{3-\delta}$-Keramikproben wurde gezeigt [6, 12], dass die Höhe und die Breite des zweiten Maximums schon durch noch kleine $\delta$-Werte $(\delta<$ 0.02) deutlich vergrößert werden kann. Die $\delta$-induzierte Verschiebung von $T_{C}$ und die Vergrößerung des ersten Maximums ist in diesem Bereich dagegen unbedeutend.

Der Elektrowiderstand ist in diesem Fall dem gut bekannten Magnetowiderstand ähnlich. Fast immer ist der ER-Effekt negativ. Es gibt aber einige $\rho(T)$-Kurven, überwiegend bei den größeren Strömen, die sich kreuzen, was auf einen positiven differentiellen ER-Effekt hinweist (seihe Abbildung 3.13). Der größte ER-Effekt findet sich immer in der Nähe des relativen Widerstandsmaximums, d.h. um die $\mathrm{T}_{\mathrm{m}}$-Temperatur herum $(134 \mathrm{~K})$. Dies ist wiederum ähnlich wie bei dem intrinsischen magnetoresistiven Effekt (MR). Vermutlich handelt es sich beim EREffekt um einen Vorgang in einem korngrenznahen Volumenbereich, da dort die E-Felder am höchsten sind. Die $\mathrm{T}_{\mathrm{m}}$-Temperatur selbst hängt nur wenig vom belastenden Strom oder vom angelegten E-Feld ab. In der Abbildung 3.15 ist die Abhängigkeit der Metall-Halbleiter Übergangstemperatur $\mathrm{T}_{\mathrm{m}}$ von dem durch die Probe fließenden Strom I aufgetragen. Man sieht, dass sich die $T_{m}(\mathrm{I})$-Kurve in zwei Teile gliedert, mit einem Grenzpunkt $\mathrm{I}=1 \mathrm{~mA}$. Beide Teile lassen sich mit einer quadratischen Funktionen beschreiben, wie gezeigt (gestrichelten Linien). Offenbar gibt es zwei verschiedene, möglicherweise räumlich getrennte Stromregime.

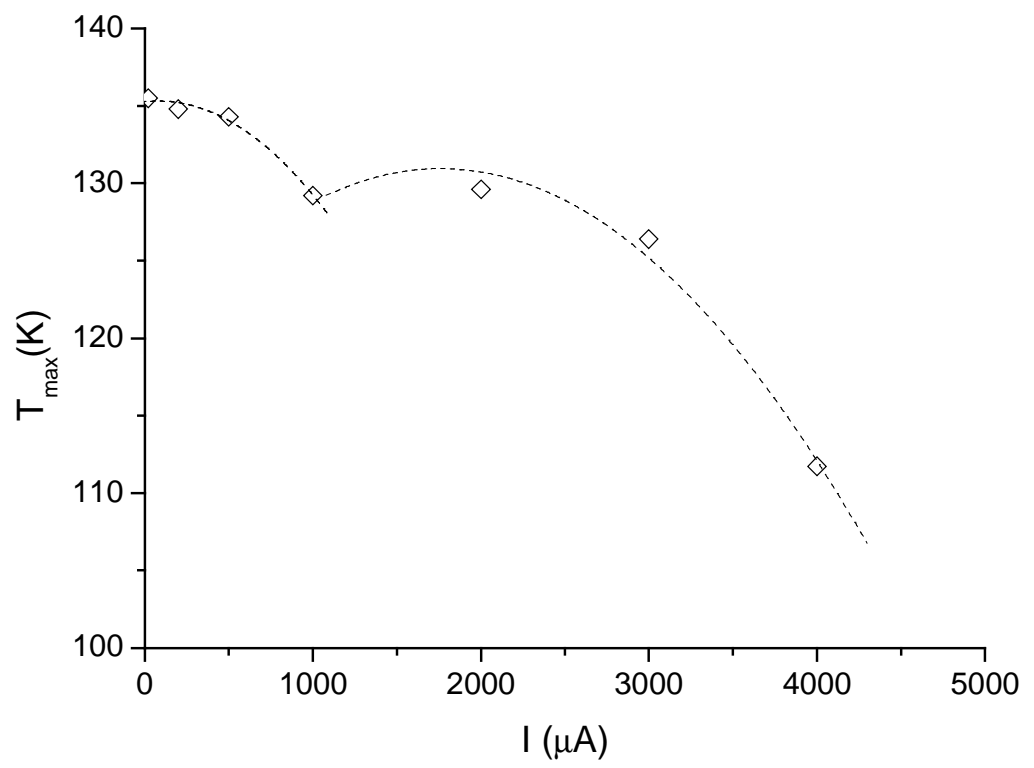

Abbildung 3.15: Temperatur des relativen Widerstandsmaximums $T_{\max }$ in Abhängigkeit vom belastenden Strom I. 
Generell gilt aber $\mathrm{dT}_{\mathrm{m}} / \mathrm{dI}<0$. Die Verschiebung von $\mathrm{T}_{\mathrm{m}}$ durch das magnetische Feld $\mathrm{dT} / \mathrm{dH}$, hat aber ein anderes Vorzeichen. Nach [14, 15] können, abhängig von der elektronischen Situation, beide Verschiebungsarten im Volumen oder im Korngrenzenbereich auftreten.

In der Abbildung 3.16 wurde das aus den R(T, I)-Kurven nach der Formel $\Delta \mathrm{R}\left(\mathrm{I}-\mathrm{I}_{0}\right) / \mathrm{R}\left(\mathrm{I}_{0}\right)\left(\mathrm{I}_{0}=20\right.$ $\mu \mathrm{A})$ ausgerechnete Elektrowiderstand bei $136 \mathrm{~K}$ gegen die verschiedenen Ströme aufgetragen. Auch hier sind zwei verschiedene Bereiche zu finden, die sich aber nun mit einer linearen Funktion anfitten lassen. Der Grenzpunkt zwischen diesen Bereichen liegt ebenfalls in der Nähe von $1 \mathrm{~mA}$. Wir haben auch den elektroresistiven Effekt unter dem magnetischen Feld $\mathrm{B}=0.5 \mathrm{~T}$ bei den Strömen I = 0.5, 1 und $4 \mathrm{~mA}$ gemessen. Der ER-Effekt wird unter einem B-Feld von 0.5 $\mathrm{T}$ kleiner, was auf eine gegenläufige Wirkung von E und B-Feldern hinweist.

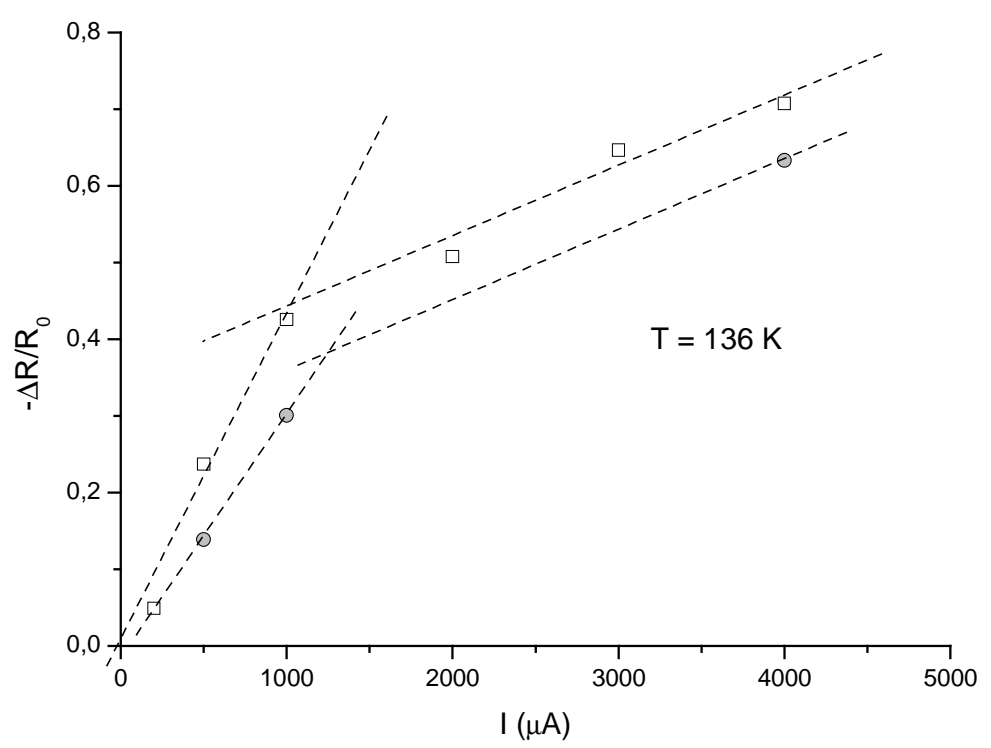

Abbildung 3.16: Betrag des elektroresistiven Effektes als Funktion des Stromes bei $T=136 \mathrm{~K}$ (der Effekt selbst ist negativ). Die gefüllte runde Symbole zeigen hier den ER-Effekt unter dem magnetischen Feld $B=0.5$ T.

Der Widerstand einer Probe mit dem Sauerstoffdefizit $\delta=0.10$ wird in der Abbildung 3.17a präsentiert. Diese Probe zeigt ebenfalls eine starke Stromabhängigkeit des Widerstandes unterhalb einer Verzweigungstemperatur $\mathrm{T}_{\mathrm{B}}$. Dieser Punkt liegt hier ungefähr bei $140 \mathrm{~K}$. Oberhalb dieser Temperatur ist die Probe ohmsch. Auch das relative Maximum $\mathrm{T}_{\max }$ liegt deutlich tiefer als vorher.

Aus dem Vergleich der Widerstandskurven mit den magnetischen Messungen sieht man, dass die schwache Struktur in der Magnetisierungskurve praktisch bei derselben Temperatur wie der Verzweigungspunk in der R(T)-Kurve zu finden ist, was wieder einen Zusammenhang zwischen Magnetisierung und Widerstandsverhalten belegt. 

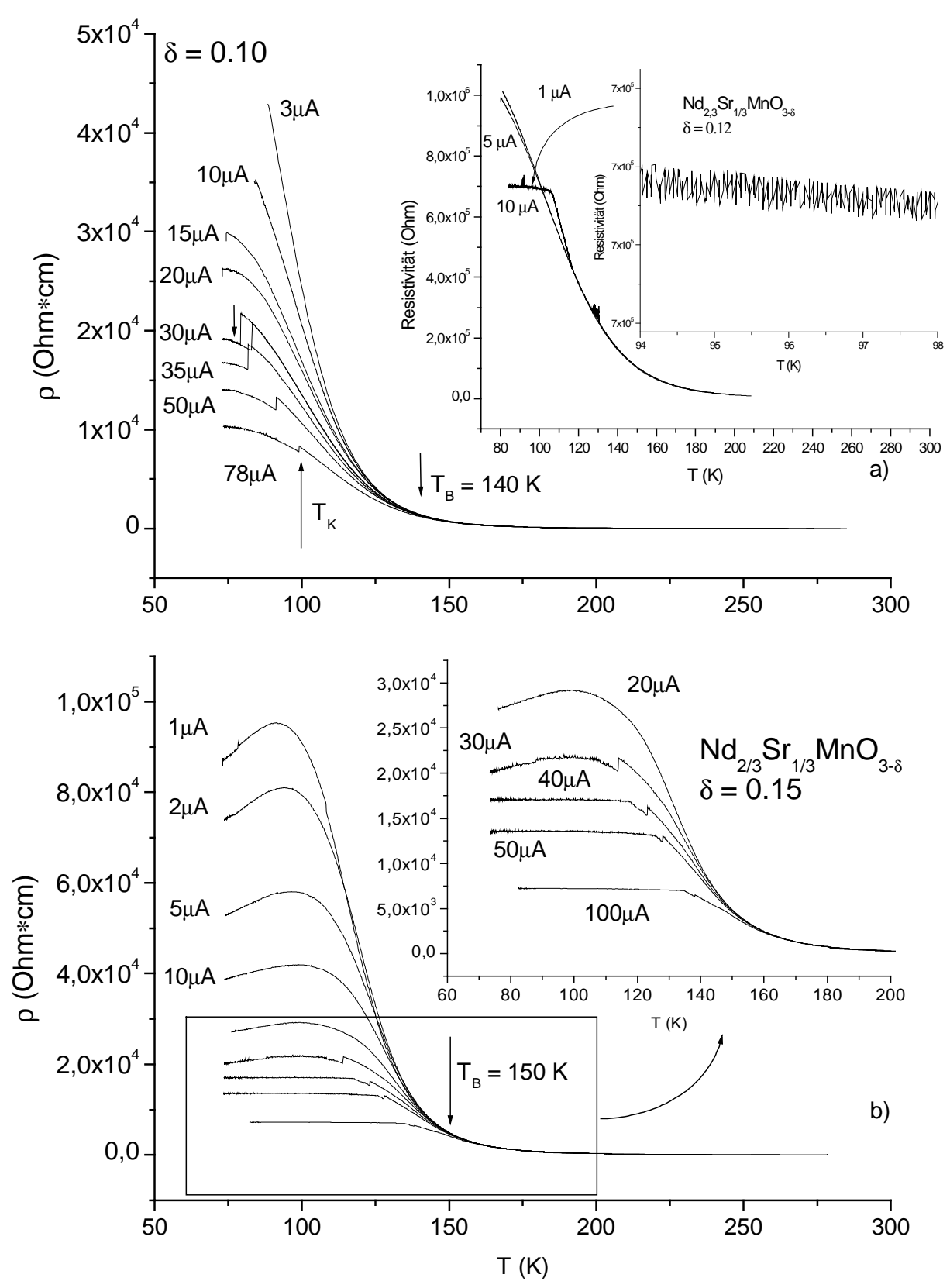

Abbildung 3.17: Die Widerstandskurven von zwei keramischen $\mathrm{Nd}_{2 / 3} \mathrm{Sr}_{1 / 3} \mathrm{MnO}_{3-\delta}$ Proben $(a: \delta=$ 0.1 und $b: \delta=0.15)$. Im Einschub sind die Widerstandskurven einer Probe mit $\delta=0.12$ aus der zweiten Probenserie gezeigt.

Diese Messung zeigt überraschenderweise im Bereich unterhalb $T_{B}$ bei den größeren Strömen eine kleine aber abrupte Anomalie bei einer Temperatur $\mathrm{T}_{\mathrm{K}}$. Diese sprungartige Anomalie ist bei kleineren Strömen nicht zu finden, d.h. die Anomalie erscheint erst bei einem kritischen Strom $I_{C}$. Die Temperatur $T_{K}$ wandert bei weiterer Stromerhöhung in Richtung höherer Temperaturen und löst sich langsam auf. Das Auftreten der sprungartigen Anomalie (mit Hysterese) legt ein kohärentes Phänomen (Phasenumwandlung) nahe. Da die $\mathrm{T}_{\mathrm{K}}$-Temperatur und das damit verbundene tiefer liegende $\rho$-Regime nur oberhalb eines bestimmten kritischen Stromes auftritt, kann man vermuten, dass wir es mit einem durch die Temperatur oder das elektrische Feld induzierten (Ordnungs-Ordnungs) Phasenübergang zu tun haben. 
Um zu prüfen, ob der gefundene ER-Effekt unabhängig von der Probenpräparation ist, haben wir eine zweite Probenserie hergestellt. In der Abbildung 3.17a (siehe Einschub) werden die Widerstandskurven der Probe mit $\delta=0.12$ aus dieser Serie gezeigt. Diese Probe zeigt ebenso einen von Strom sehr stark abhängiges Widerstandsverhalten. Zusätzlich findet man bei den Strom I = $10 \mu \mathrm{A}$ einen Bereich, in dem der Widerstand instabil ist (,Schalteffekt“, siehe Anhang A). Auch in der Literatur gibt es vereinzelte Berichte über ein ungewöhnliches R-Verhalten von Manganitproben [9], jedoch ist dort die Systematik und Interpretation noch sehr vorläufig.

In der Abbildung 3.17b werden die Widerstandsmessungen an einer Probe mit $\delta=0.15$ dargestellt. Diese Probe zeigt ein ähnliches Verhalten wie die Probe mit $\delta=0.1$. Der $\mathrm{T}_{\mathrm{B}}$-Punkt liegt ungefähr bei $150 \mathrm{~K}$, d.h. hier höher als bei der Probe mit $\delta=0.1$. Die Maximumtemperatur $\mathrm{T}_{\max }$ liegt auch etwas höher als bei der Probe mit dem kleineren nominalen Sauerstoffdefizit. Die Maximumtemperatur $\mathrm{T}_{\max }$ verschiebt sich mit der Stromerhöhung, allerdings in die andere Richtung als bei den Proben mit kleinen $\delta\left(\mathrm{dT}_{\max } / \mathrm{dI}>0\right)$. Ein solches Verhalten zeigt uns wieder (ähnlich wie die magnetischen Messungen), dass bei den Proben mit $\delta>0.07$ ein neues Regime auftritt.

In der Abbildung 3.18 ist die V(I)-Charakteristik der Probe mit $\delta=0.15$ bei einer Temperatur unterhalb des $\mathrm{T}_{\mathrm{B}}$-Punktes zu sehen. Man sieht auch hier eine sprungartige Anomalie, aber nun gegen den Strom I. Diese Anomalie hat ebenfals eine deutliche Hysterese. Oberhalb der $\mathrm{T}_{\mathrm{B}^{-}}$ Temperatur sind die Kennlinien linear, wie bei allen anderen Proben.

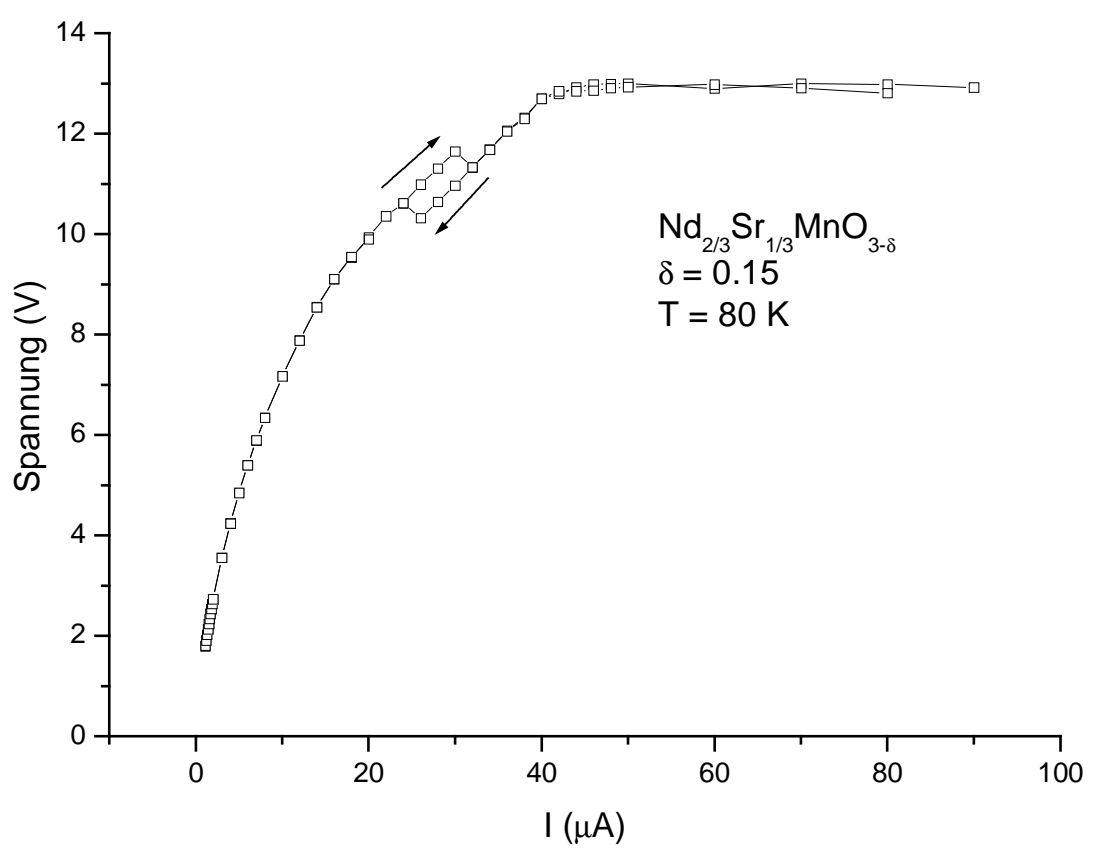

Abbildung 3.18: Die an der $\mathrm{Nd}_{2 / 3} \mathrm{Sr}_{1 / 3} \mathrm{MnO}_{2.85}$-Probe gemessene $\mathrm{V}(\mathrm{I})$-Kennlinie $(\mathrm{T}=80 \mathrm{~K})$.

In den $\rho$ (T)-Kurven bei der Probe mit $\delta=0.15$ (Abb. 3.17b ), unterhalb des $T_{K}$-Punktes (d.h. bei den höheren Strömen) findet man einen Bereich, in dem die abgegriffene Spannung und damit auch der Probenwiderstand konstant bleibt. Ein ähnliches Verhalten zeigt auch die V(I)- 
Kennlinie bei den höheren Strömen (siehe Abbildung 3.18). Dies könnte man eine VaristorKennlinie nennen.

Der elektroresistive Effekt für diese zwei Proben $(\delta=0.10, \mathrm{~T}=100 \mathrm{~K}$ und $\delta=0.15, \mathrm{~T}=92 \mathrm{~K})$ ist der Abbildung 3.19 zu entnehmen. Der ER-Effekt wurde ähnlich wie bei der Probe mit $\delta=$ 0.05 aus den $\rho(\mathrm{T})$-Kurven berechnet.
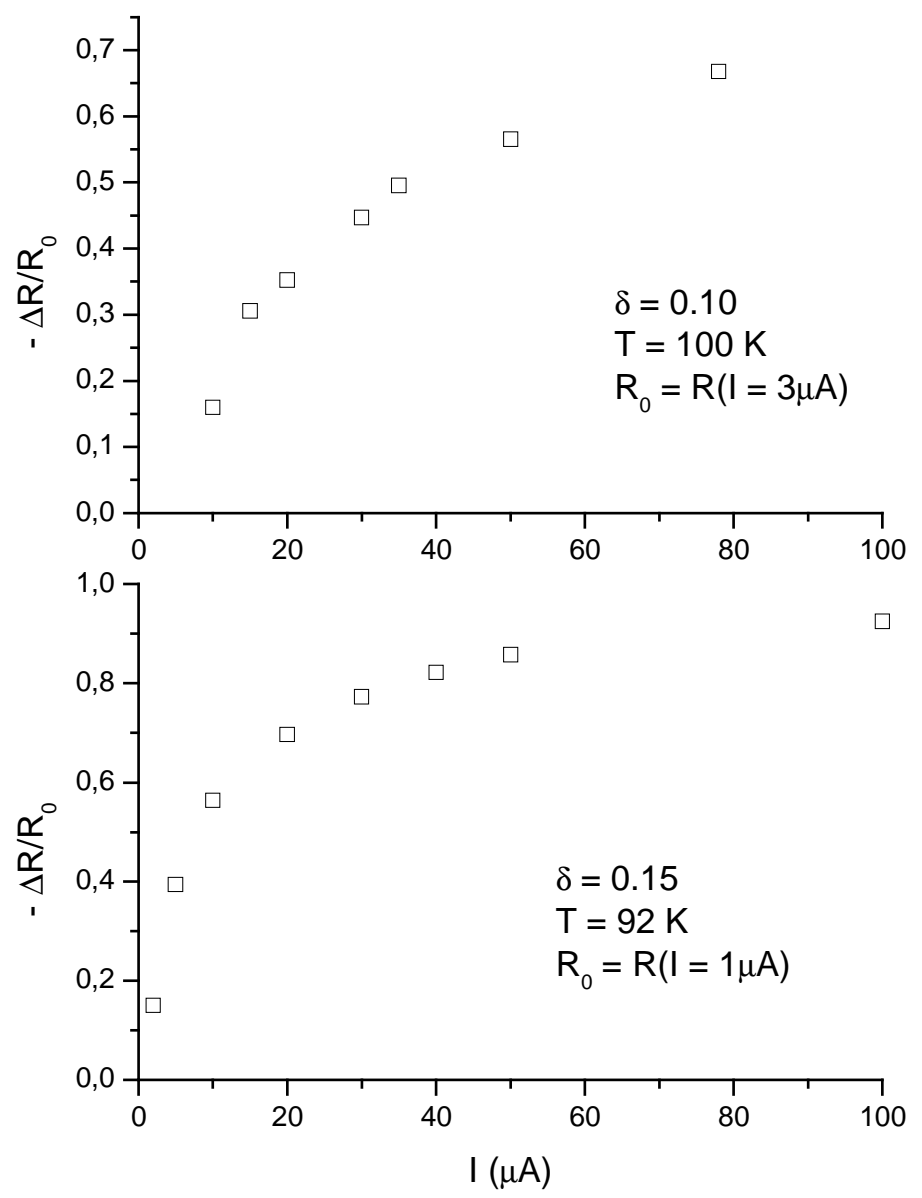

Abbildung 3.19: Elektroresistiver Effekt bei der Probe mit $\delta=0.10$ (oben) und $\delta=0.15$ (unten) als Funktion des Stromes. Insbesondere wurde der ER-Effekt der Probe mit dem Sauerstoffdefizit $\delta=0.15$ bei der Temperatur des Maximums $T_{\max }=92 \mathrm{~K}$ und bezogen auf den Strom $I=1 \mu \mathrm{A}$ berechnet. Für den Null-Widerstand $R_{0}$ wurde der Probenwiderstand $R$ bei $92 \mathrm{~K}$ und $1 \mu \mathrm{A}$ genommen. Da das Maximum des Widerstandes der Probe mit $\delta=0.10$ deutlich tiefer als Stickstofftemperatur liegt, haben wir den ER-Effekt dort bei einer Temperatur $(T=100 \mathrm{~K})$ direkt oberhalb des $T_{K}$-Punktes bei Strom $I=78 \mu$ A berechnet. Der Null-Widerstand $R_{0}$ ist hier der Probenwiderstand, gemessen bei $I=3 \mu \mathrm{A}$.

Der elektroresistive Effekt zeigt eine Tendenz, mit zunehmenden Sauerstoffdefizit $\delta$ stärker zu werden. Der ER-Effekt zeigt außerdem ein Sättigungsverhalten. Die Probe mit $\delta=0.15$ hat praktisch den Endwert schon bei einem Strom von $\mathrm{I}=60 \mu \mathrm{A}$ erreicht. Es gilt also, je größer $\delta$ ist, desto größer ist der Sättigungswert und desto schneller erreichen wir ihn. 
Die Widerstandsmessungen an der nächsten Probe $(\delta=0.20)$ werden in Abbildung 3.20 präsentiert. Die Verzweigungstemperatur $\mathrm{T}_{\mathrm{B}}$ liegt bei dieser Probe bei einer ziemlich hohen Temperatur (ungefähr bei $200 \mathrm{~K}$ ). Das stimmt überein mit der steigenden Tendenz der Verzweigungstemperatur im zweiten Regime $(\delta>0.07)$. Bei tiefen Temperaturen ist diese Probe stark isolierend, und das Widerstandsmaximum zeigt sich nur noch als eine Schulter. Die Schulter ist bei größeren Strömen kaum mehr zu sehen. Wenn man versucht, die Lage dieser Schulter zu identifizieren und dem Maximum bei den anderen Proben zuzuordnen, sieht man, dass diese Temperatur $(\approx 125 \mathrm{~K})$ höher liegt als bei der vorherigen Probe $(\delta=0.15)$. Auch dies bestätigt wieder das zweite $\delta$-Regime. Ein zweites (inneres) Probenstück zeigt die starke Isolatoreigenschaft noch nicht, sondern ist eher der Probe mit $\delta=0.15$ ähnlich. Das weist auf eine ziemlich breite Verteilung von $\delta$ bei hohen Werten hin.

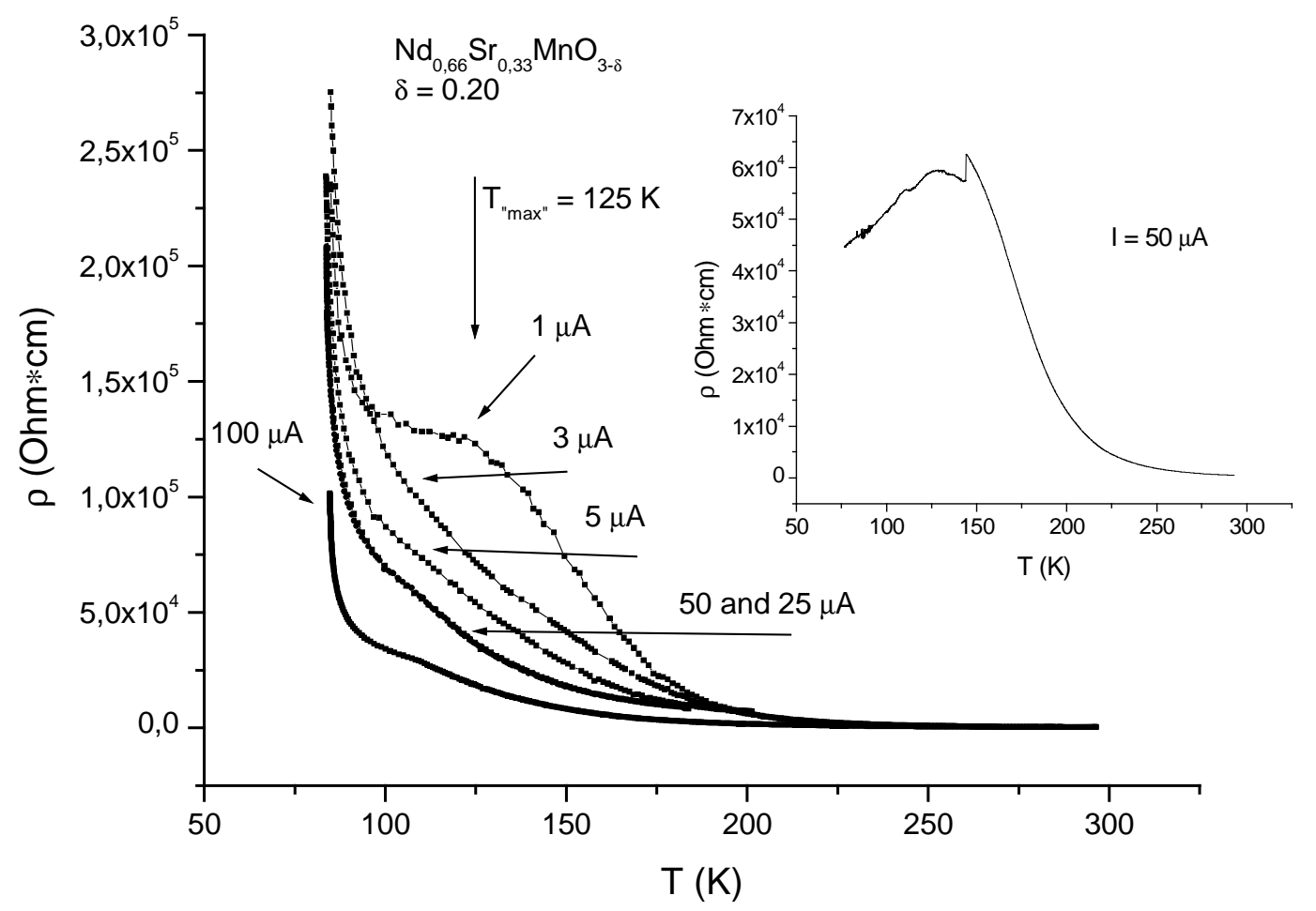

Abbildung 3.20: Spezifischer Widerstand der keramischen $\mathrm{Nd}_{2 / 3} \mathrm{Sr}_{1 / 3} \mathrm{MnO}_{2.8}$-Probe, gemessen bei verschiedenen Strombelastungen (Einschub: anderes Probenstück-Probe B).

\subsection{Elektrowiderstand der $\mathrm{Nd}_{2 / 3} \mathrm{Sr}_{1 / 3} \mathrm{MnO}_{3-\delta}-\mathrm{Schichtproben}$}

Widerstandsmessungen unter verschiedenen Strombelastungen wurden auch an den $\mathrm{Nd}_{2 / 3} \mathrm{Sr}_{1 / 3} \mathrm{MnO}_{3-\delta}$-Schichtproben durchgeführt, zum Teil um zu prüfen, ob elektroresistive Effekte auch im Volumen existieren. Die Schichtproben haben üblicherweise weit weniger Korngrenzen. Das bedeutet aber auch, dass sich der ER-Effekt bei diesen Proben vom Effekt bei den keramischen Proben unterscheiden sollte. Bei der Keramik erwarten wir, dass die Korngrenzen eine größere Rolle spielen. 
Die Widerstandskurven der vier $\mathrm{Nd}_{2 / 3} \mathrm{Sr}_{1 / 3} \mathrm{MnO}_{3-\delta}$-Schichtproben werden in der Abbildung 3.21 präsentiert.
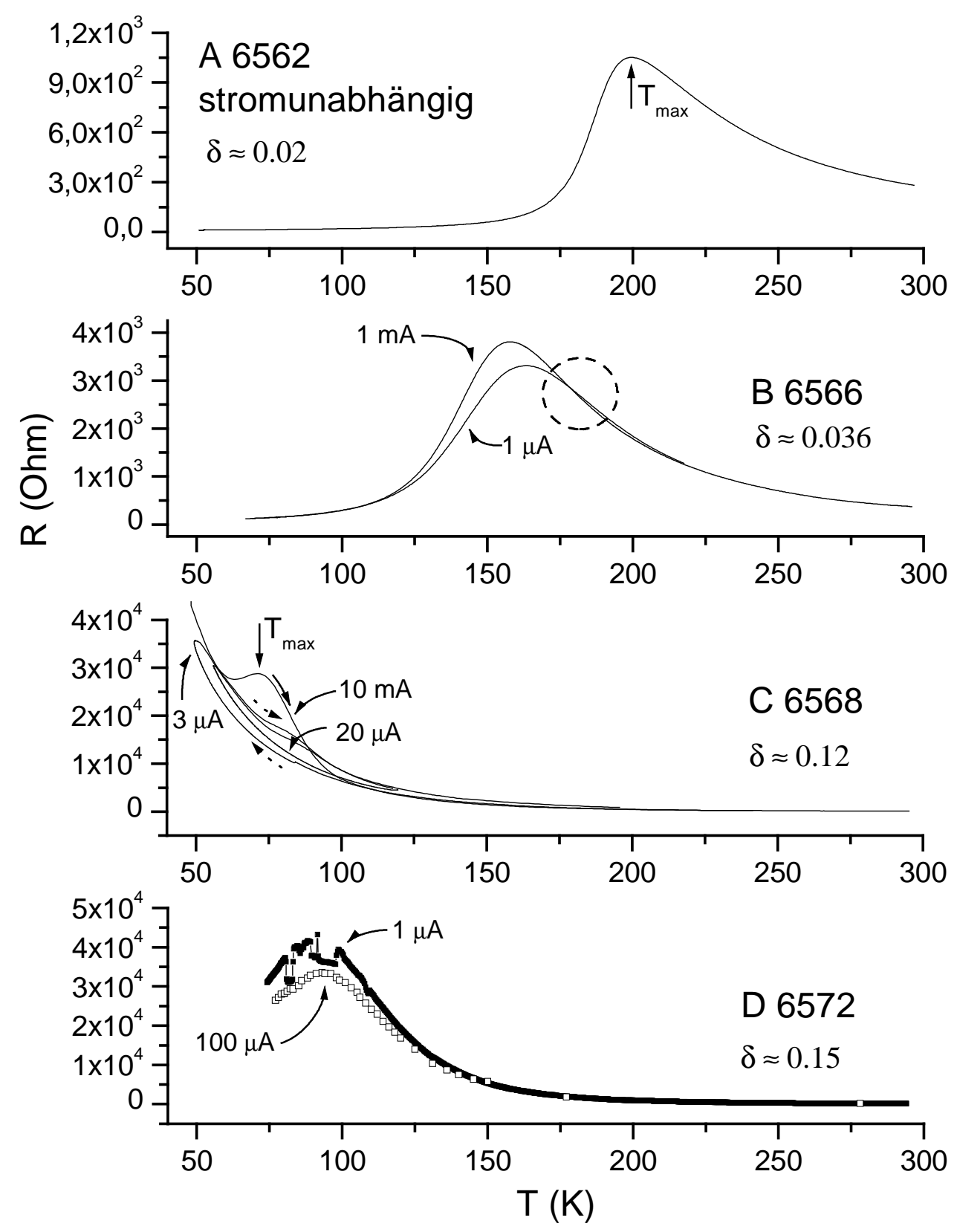

Abbildung 3.21: Die bei festem Strom gemessene Widerstandskurven der vier $\mathrm{Nd}_{2 / 3} \mathrm{Sr}_{1 / 3} \mathrm{MnO}_{3-\delta^{-}}$ Schichtproben $(a: \delta \approx 0.02, b: \delta \approx 0.36, c: \delta \approx 0.12, \delta \approx 0.15$ ).

Durch die R(T)-Messungen bei verschiedenen Strömen bekommen wir nun zunächst eine Möglichkeit, den Sauerstoffdefizit dieser Proben abzuschätzen. Der Sauerstoffgehalt wurde aus dem Vergleich der Temperaturen des Widerstandsmaximums $\mathrm{T}_{\max }$ (beim kleinsten Strom gemessen) mit den entsprechenden Temperaturen bei den keramischen Proben ermittelt. $\delta$ für die keramischen Proben wurde aus dem Massenverlust bestimmt (siehe Kapitel „Experimentelles“). In der Abbildung 3.22 ist ein $\mathrm{T}_{\max }-\delta$-Diagramm gezeigt. Die zwei ersten Proben (A und B) haben einen Metall-Isolator-Übergang $\mathrm{T}_{\max }$ bei 199 und $163 \mathrm{~K}$. Aus der Interpolation bekommen wir für die Probe $\mathrm{A}, \delta \approx 0.02$ und für die Probe $\mathrm{B}, \delta \approx 0.036$. Die Probe $\mathrm{D}$ zeigt ein Maximum bei 91 $\mathrm{K}$, was mit der keramischen Probe mit $\delta=0.15$ völlig identisch ist. Für die Probe $\mathrm{C}$ kann $\delta$ nur 
zwischen 0.036 und 0.15 liegen (siehe Abb. 3.22). Aus der linearen Extrapolation erhalten wir $\delta$ $\approx 0.12$. Die abgeschätzten $\delta$-Werten stimmten mit den Präparationsbedingungen gut überein, denn je ärmer die Kammer-Atmosphäre an Sauerstoff war, desto größere $\delta$-Werte bekommen wir. Das gemeinsame $\mathrm{T}_{\max }-\delta$-Diagramm zeigt, dass auch bei der Schichtserie zwei unterschiedliche $\delta$-Regime existieren.

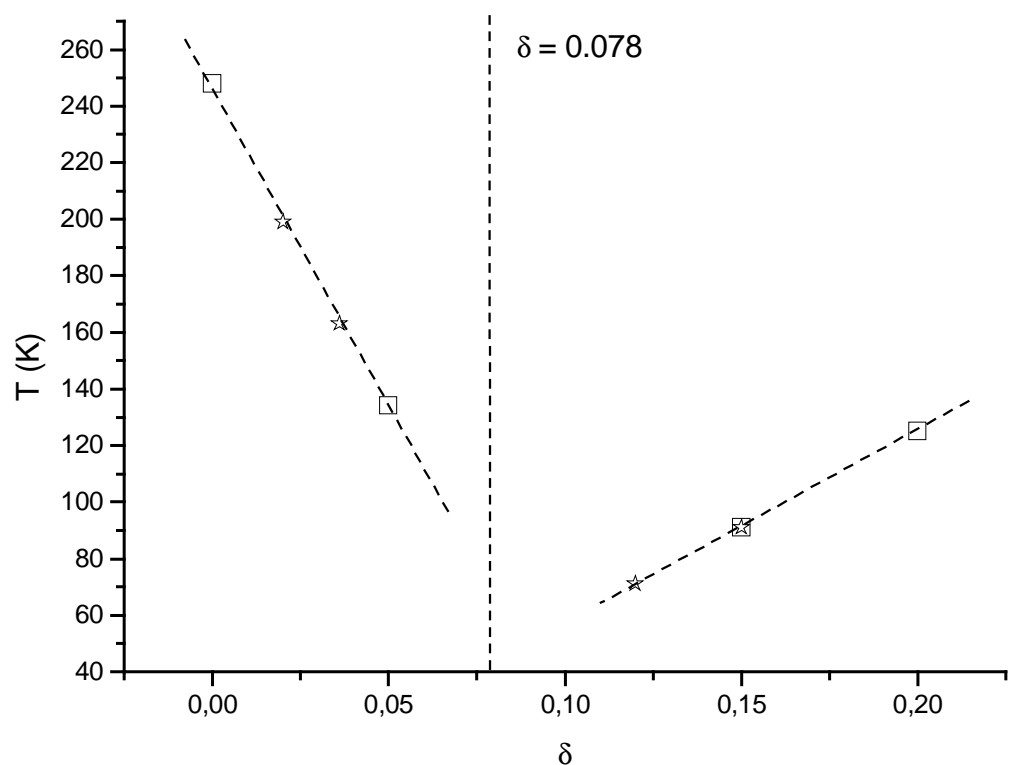

Abbildung 3.22: Zur $\delta$-Abschätzung für die $\mathrm{Nd}_{2 / 3} \mathrm{Sr}_{1 / 3} \mathrm{MnO}_{3-\delta}$ Schichtproben: die $T$ - $\delta$-Diagramm (die rechteckigen Symbole entsprechen der Serie 1, die Sternsymbole sind mit Hilfe der Extrapolation (bzw. Interpolation) erhaltene Werte für die Schichtproben). Die gestrichelte Linie zeigt ungefähr die Grenze zwischen den zwei $\delta$-Regimen (aus den magnetischen Messungen an den keramischen Proben gewonnen).

Die Schichtproben zeigen auch einen elektroresistiven Effekt. Es gibt jedoch Unterschiede zu den keramischen Proben. Die Probe mit dem kleinsten Wert, $\delta \approx 0.02$, ist noch der keramischen Probe mit $\delta=0$ ähnlich. Der Widerstand dieser Probe zeigt keine Abhängigkeit vom eingeprägten Strom.

Der Widerstand der Probe mit $\delta \approx 0.036$ hängt schon deutlich vom Messstrom ab (siehe Abbildung 3.21). Die zwei gemessenen $\mathrm{R}(\mathrm{T})$-Kurven ( $=1 \mu \mathrm{A}$ und $1 \mathrm{~mA}$ ) zeigen in der Nähe des Widerstandsmaximums einen positiven ER-Effekt. Die Abbildung 3.21 zeigt aber auch, dass der ER-Effekt sein Vorzeichen wechseln kann. Die Widerstandskurven überschneiden sich gelegentlich. Einer dieser Überschneidungspunkte bei $\mathrm{T} \approx 175 \mathrm{~K}$ ist in der Abbildung ausgezeichnet (Kreis). Bei ganz hohen und ganz tiefen Temperaturen geht der ER-Effekt kontinuierlich gegen Null. Der maximale Wert des elektroresistiven Effektes liegt bei 27\% (I = 1 $\mu \mathrm{A}$ und 1mA). Zum Vergleich: der absolute Wert des ER-Effektes bei der keramischen Probe mit $\delta=0.05$ für den Strom I = 1mA beträgt 43\%. Damit sehen wir, dass die Schichtproben bei gleichen Bedingungen einen etwas kleineren ER-Effekt zeigen. 

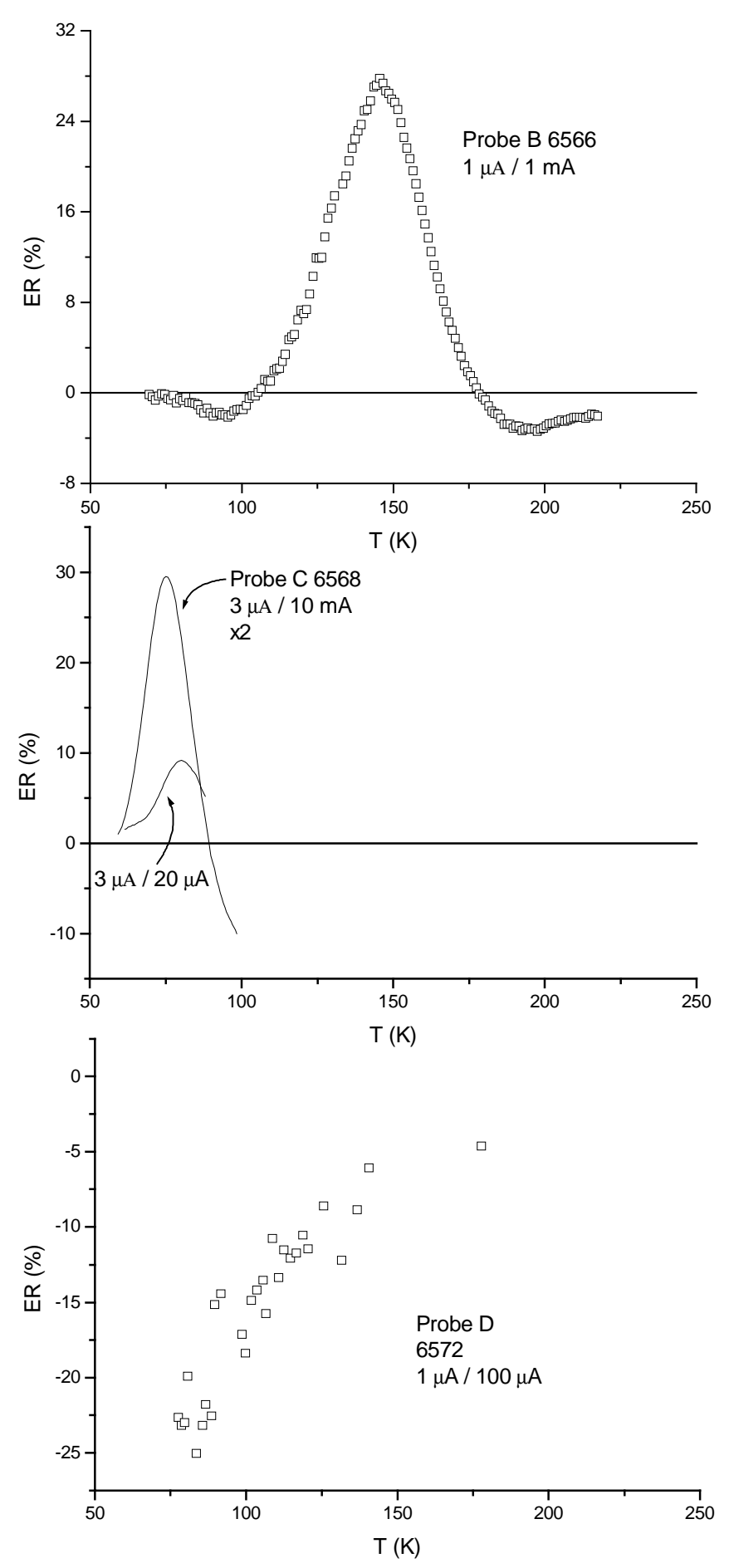

Abbildung 3.23: Der Elektrowiderstand der $\mathrm{Nd}_{2 / 3} \mathrm{Sr}_{1 / 3} \mathrm{MnO}_{3-\delta}$ Schichtproben.

Bei der Probe mit $\delta=0.05$ wurde der ER-Effekt als eine Überlagerung von Effekten aus Korngrenzen und Volumen betrachtet. Damit stellt sich nun die Frage, ob bei den Schichten das Volumen oder eventuelle innere Grenzflächen die wichtigere Rolle spielen. Die Tatsache, dass diese Probe eine etwas schwächeren und positiven ER-Effekt zeigt, könnte man so deuten, dass hier der elektroresistiven Effekt überwiegend im Volumen entsteht. Bei den keramischen Proben finden wir einen positiven ER-Effekt nur bei größeren Strömen, wo der Korngrenzeneffekt möglicherweise schon durch zunehmende Injektion über die Korngrenze verschwunden ist. 
Die Schichtprobe $\mathrm{C}$ mit $\delta \approx 0.12$ zeigt ebenfalls eine positiven Elektrowiderstand, dessen Maximum wiederum in der Nähe des Maximums der R(T)-Kurven liegt. Bei höheren Temperaturen gibt es hier auch den Übergang vom positiven zum negativen ER-Effekt. Der absolute Wert des elektroresistiven Effektes (ausgerechnet für die Ströme I $=3 \mu \mathrm{A}$ und $20 \mu \mathrm{A}$ ) ist ebenfalls kleiner als bei vergleichbaren keramischen Proben bei derselben Strombelastung. Dort liegen die vergleichbare Werte bei $\approx 35 \%(\delta=0.10)$ und $\approx 70 \%(\delta=0.15)$.

Die Schichtprobe mit $\delta \approx 0.15$ ist sehr der keramischen Probe mit $\delta=0.15$ ähnlich. Auch der ER-Effekt bleibt bei dieser Probe immer negativ. Der maximale gemessene ER-Effekt ist aber viel kleiner als bei der entsprechenden keramischen Probe, wo er ungefähr bei $90 \%$ liegt. Zusätzlich zeigt die $\mathrm{R}(\mathrm{T})$-Kurve (gemessen bei $\mathrm{I}=1 \mu \mathrm{A}$, siehe Abb. 3.21d), dass bei dieser Probe Widerstandsinstabilitäten auftreten. Diese Schalteffekte und der negative Elektrowiderstand weisen darauf hin, dass auch in dieser Probe irgendwelche innere Grenzen vorhanden sind. Die Präparationsbedingungen bestätigen diese Aussage. Da die Probe bei ganz geringem Sauerstoffpartialdruck hergestellt wurde, erwartet man eine Vielzahl von Strukturdefekten, die sich zu inneren Grenzen anordnen könnten (Korngrenzen oder Zwillingsgrenzen).

Die Schichtproben sind also der keramischen Proben durchaus ähnlich. Sie zeigen einen EREffekt, der mit größeren $\delta$ auch größer wird. Man sieht bei den Schichten aber meist einen positiven Elektrowiderstand, der bei der Keramik nur zu finden ist, wenn die Korngrenzen bereits deaktiviert wurden. Es gibt also offenbar einen ER-Effekt sowohl an den DomänenGrenzen als auch im Inneren der Domänen, meist aber mit verschiedenen Vorzeichen.

\subsection{Magnetowiderstand der keramischen $\mathrm{Nd}_{2 / 3} \mathrm{Sr}_{1 / 3} \mathrm{MnO}_{3-\delta}-\operatorname{Proben}$}

Eine der wichtigsten Eigenschaften der Manganite ist der Magnetowiderstand (MR-Effekt), der als $M R=\Delta R / R_{0}\left(\Delta R=R_{H \neq 0}-R_{0}, R_{0}\right.$ ist der Probenwiderstand im Feld $\left.H=0\right)$ definiert ist. Der Magnetowiderstand wurde hier unter Magnetfeldern bis zu 0.5 T gemessen. Der unter dem Feld $\mathrm{B}=0.5 \mathrm{~T}$ gemessene MR-Effekt der stoichiometrischen $\mathrm{Nd}_{2 / 3} \mathrm{Sr}_{1 / 3} \mathrm{MnO}_{3}$-Probe ist in Abbildung 3.24a gezeigt. Dieses Ergebniss ist nahe den Literaturdaten [17]. Der Magnetowiderstand ist negativ und zeigt bei $240 \mathrm{~K}$ ein Maximum. Zusätzlich ist eine schwache Struktur (ein zweites Maximum) bei $170 \mathrm{~K} \mathrm{zu}$ sehen. Dieses Maximum kann dem Korngrenzenbereich zugeordnet werden, ist aber offenbar kein Tunneleffekt, da er nicht kontinuierlich verläuft [105]. Der Magnetowiderstand wurde auch mit verschiedenen Messströmen untersucht. Die Abbildung 3.24b zeigt die Abhängigkeit des MR-Effektes vom magnetischen Feld bei den Strömen I = 0.5, 1, 2 und $4 \mathrm{~mA}$. Alle vier Kurven unterscheiden sich nur wenig, d.h. das elektrische Feld beeinflusst die stoichiometrische Probe fast nicht.

Die Abbildung 3.25 zeigt den MR-Effekt der $\mathrm{Nd}_{2 / 3} \mathrm{Sr}_{1 / 3} \mathrm{MnO}_{3-\delta}$-Probe mit $\delta=0.05$. Die Probe wurde bei den Strömen I $=1 \mu \mathrm{A}, 0.5,1$ und $4 \mathrm{~mA}$ untersucht. Man sieht sofort, dass hier der Magnetowiderstand vom eingeprägten Strom stark abhängig ist. Die Messungen des 
elektroresistiven Effektes haben gezeigt, dass diese Probe zwei Stromregime mit der Grenze I = $1 \mathrm{~mA}$ nahelegt, siehe Kapitel „Spezifischer Widerstand und V(I)-Charakteristiken“. Um zu prüfen, ob der Magnetowiderstand auch diese zwei Stromregime aufdeckt, haben wir auch die Abhängigkeit des Magnetowiderstandes vom Strom bei verschiedenen festen Magnetfeldern (B $=0.075,0.25$ und $0.5 \mathrm{~T}$ ) untersucht. In Abbildung 3.26 sind diese Messungen zusammengefasst.
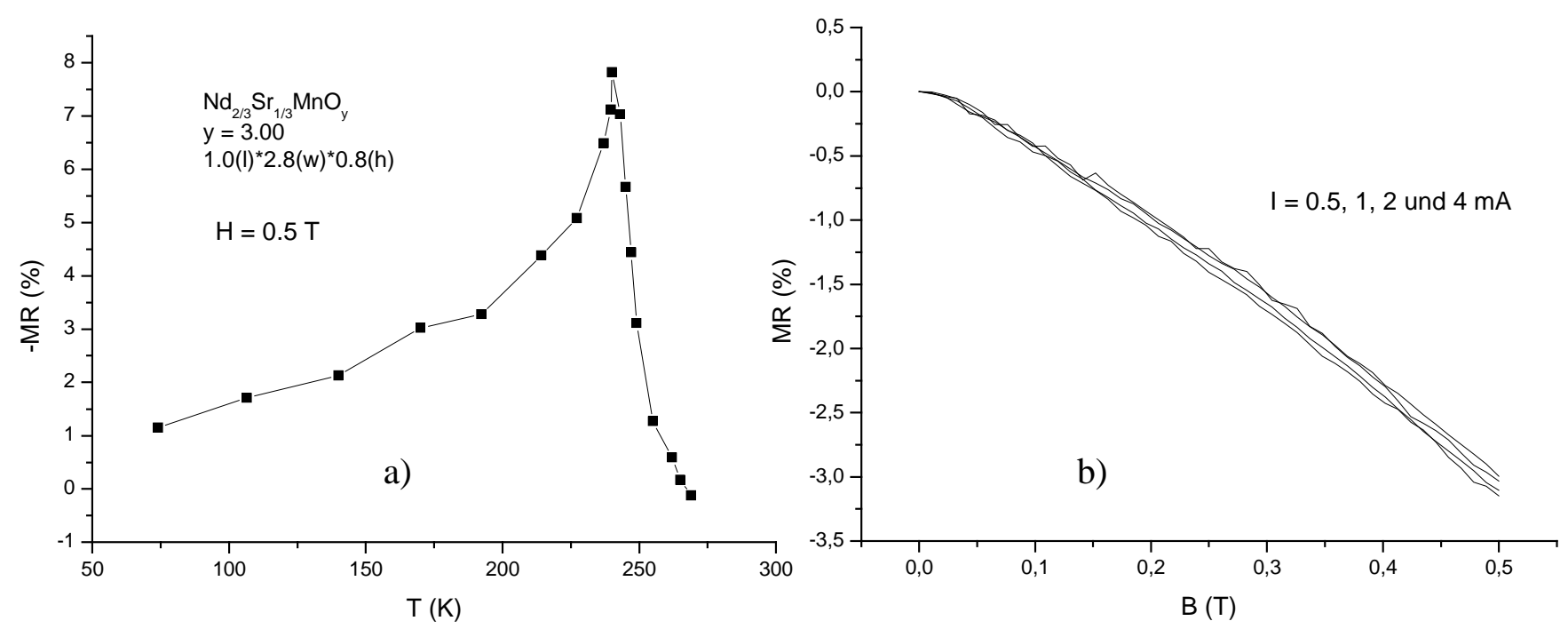

Abbildung 3.24: Der Magnetowiderstand der $\mathrm{Nd}_{2 / 3} \mathrm{Sr}_{1 / 3} \mathrm{MnO}_{3-\delta}$ Probe $(\delta=0)$ als Temperatur(a) und Feldfunktion (b).

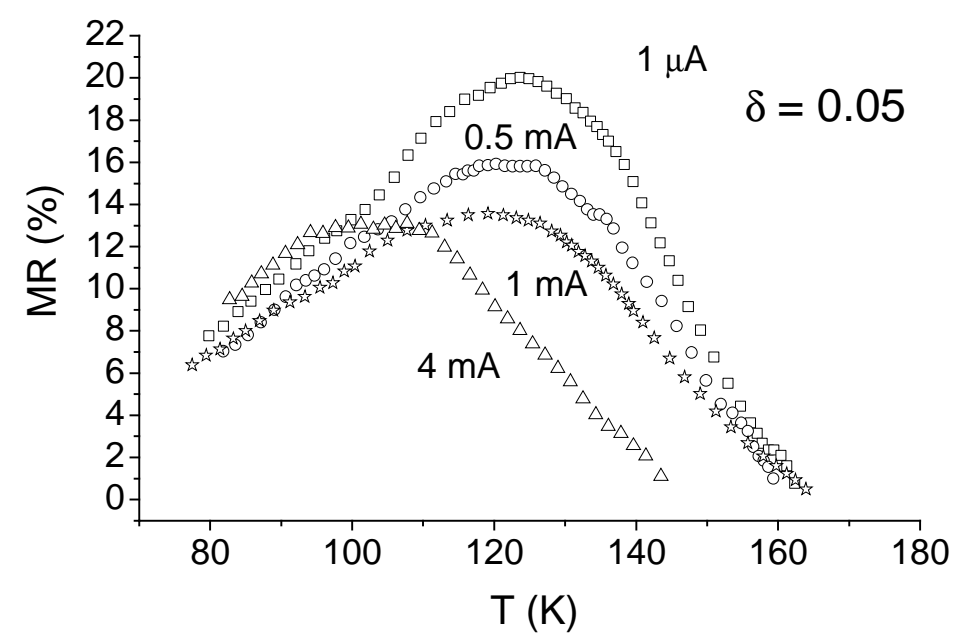

Abbildung 3.25: Der Magnetowiderstand der $\mathrm{Nd}_{2 / 3} \mathrm{Sr}_{1 / 3} \mathrm{MnO}_{3-\delta}$ Probe $(\delta=0.05)$, gemessen unter verschiedenen Strombelastungen.

Allgemein gilt die Regel, dass mit der Erhöhung des magnetischen Feldes der Magnetowiderstand größer wird. Bei allen bei $77 \mathrm{~K}$ gemessenen MR(I)-Kurven (Abbildung 3.26a) kann man eine Anomalie bei Strom $I \approx 1 \mathrm{~mA}$ finden. Diese Anomalie befindet sich in guter Korrelation mit der Anomalie der ER-Messungen, so dass sie einer Grenze zwischen zwei Stromregimen zugeordnet werden kann. Die Messung bei der Temperatur T = $122 \mathrm{~K}$, d.h. noch 
unterhalb der Maximum der $\rho(\mathrm{T})$-Kurve $\mathrm{T}_{\max }$, zeigt bei dem kleinsten Strom und bei $\mathrm{B}=0.075 \mathrm{~T}$ überraschenderweise einen Vorzeichenwechsel des MR bei einem Strom von $\mathrm{I} \approx 2 \mathrm{~mA}$. Die weiteren Kurven $(B=0.25$ und $0.5 \mathrm{~T})$ zeigen einen stärkeren MR-Effekt, der aber immer negativ bleibt. In der Nähe von $1 \mathrm{~mA}$, wo wir die mit den zwei Strombereichen verbundene Anomalie erwarten, zeigt sich aber immer noch eine schwache Struktur, ähnlich der Anomalie bei der $77 \mathrm{~K}$ - Messung. Alle drei Kurven zeigen ein Maximum bei ungefähr $3 \mathrm{~mA}$. Die bei der Temperatur $\mathrm{T}=128 \mathrm{~K}$ gemessenen Kurven sind der Messung bei $\mathrm{T}=122 \mathrm{~K}$ ähnlich. Die Anomalie beim Strom $\mathrm{I}=1 \mathrm{~mA}$ ist aber kaum noch zu sehen. Ein positiver Magnetowiderstand wird auch hier nicht erreicht. Die Abbildung 3.26d zeigt auch noch den Magnetowiderstand bei der Temperatur $\mathrm{T}=140 \mathrm{~K}$ (rechts vom Widerstandsmaximum) und unter dem magnetischen Feld B = $0.5 \mathrm{~T}$; dort sind kaum noch Strukturen sichtbar.
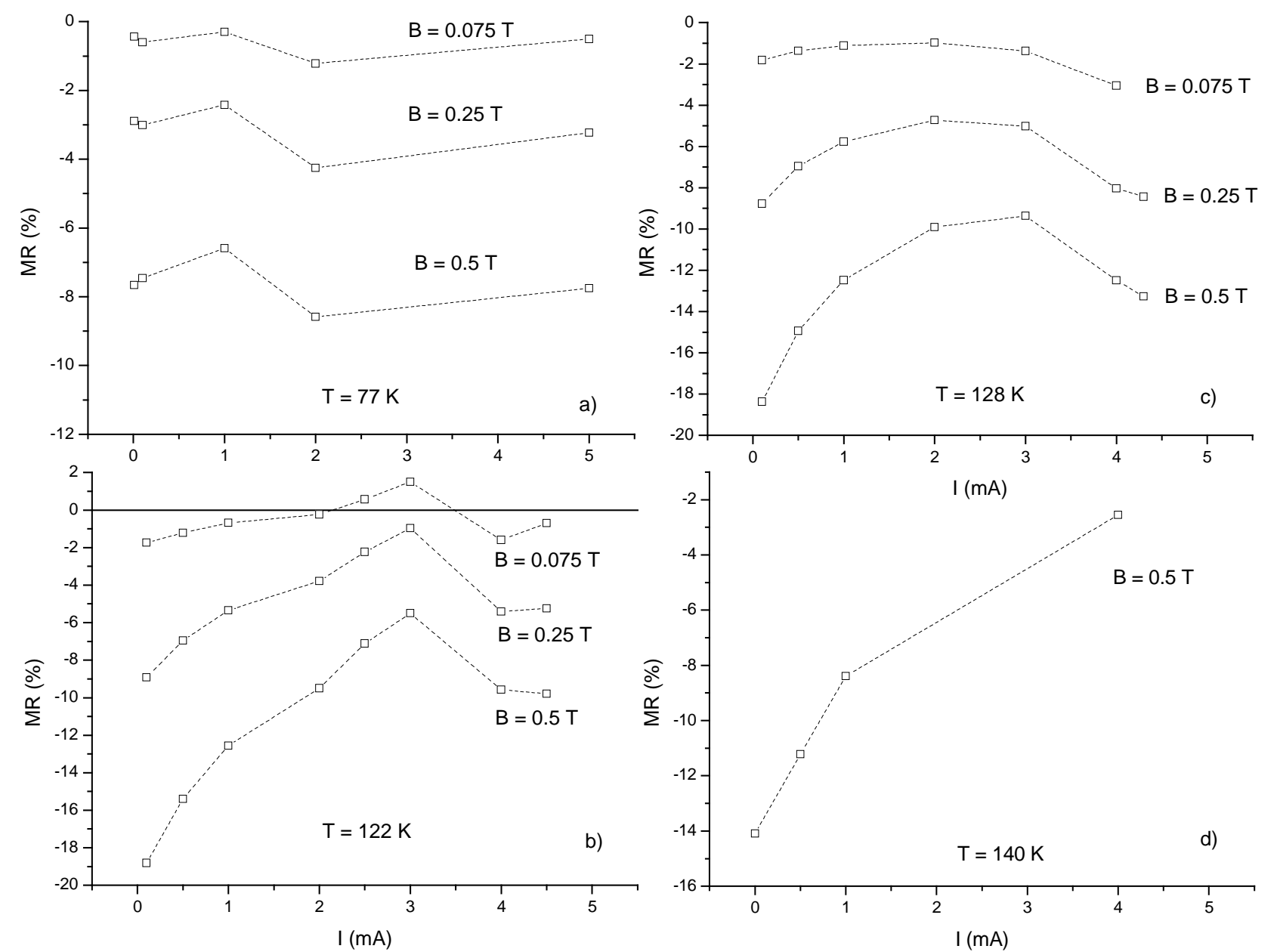

Abbildung 3.26: Die Abhängigkeit des magnetoresitiven Effektes von der Strombelastung bei jeweils festem $\mathrm{B}$-Feld $\left(\mathrm{Nd}_{2 / 3} \mathrm{Sr}_{1 / 3} \mathrm{MnO}_{3-\delta}, \delta=0.05\right)$.

Der Magnetowiderstand der $\mathrm{Nd}_{2 / 3} \mathrm{Sr}_{1 / 3} \mathrm{MnO}_{3-\delta}$-Probe mit $\delta=0.10$ als Funktion des Stromes bei drei verschiedenen Temperaturen und unter dem magnetischen Feld $\mathrm{B}=0.5 \mathrm{~T}$ ist in der Abbildung $3.27 \mathrm{zu}$ sehen. Der absolute MR-Effekt bei dieser Probe ist schon wesentlich keiner als bei der Probe mit $\delta=0.05$. Alle drei Kurven (bei $\mathrm{T}=76,118$ und $131 \mathrm{~K}$ ) zeigen hier ein Sättigungsverhalten. Im Prinzip sehen die MR-Kurven ähnlich aus wie die ER-Kurven (siehe 
auch Kapitel „Spezifischer Widerstand und V(I)-Charakteristiken“). Sowohl beim Elektrowiderstand als auch beim Magnetowiderstand kann man wieder zwei Kurventeile erkennen, mit dem Grenzstrom $\mathrm{I} \approx 25 \mu \mathrm{A}$.

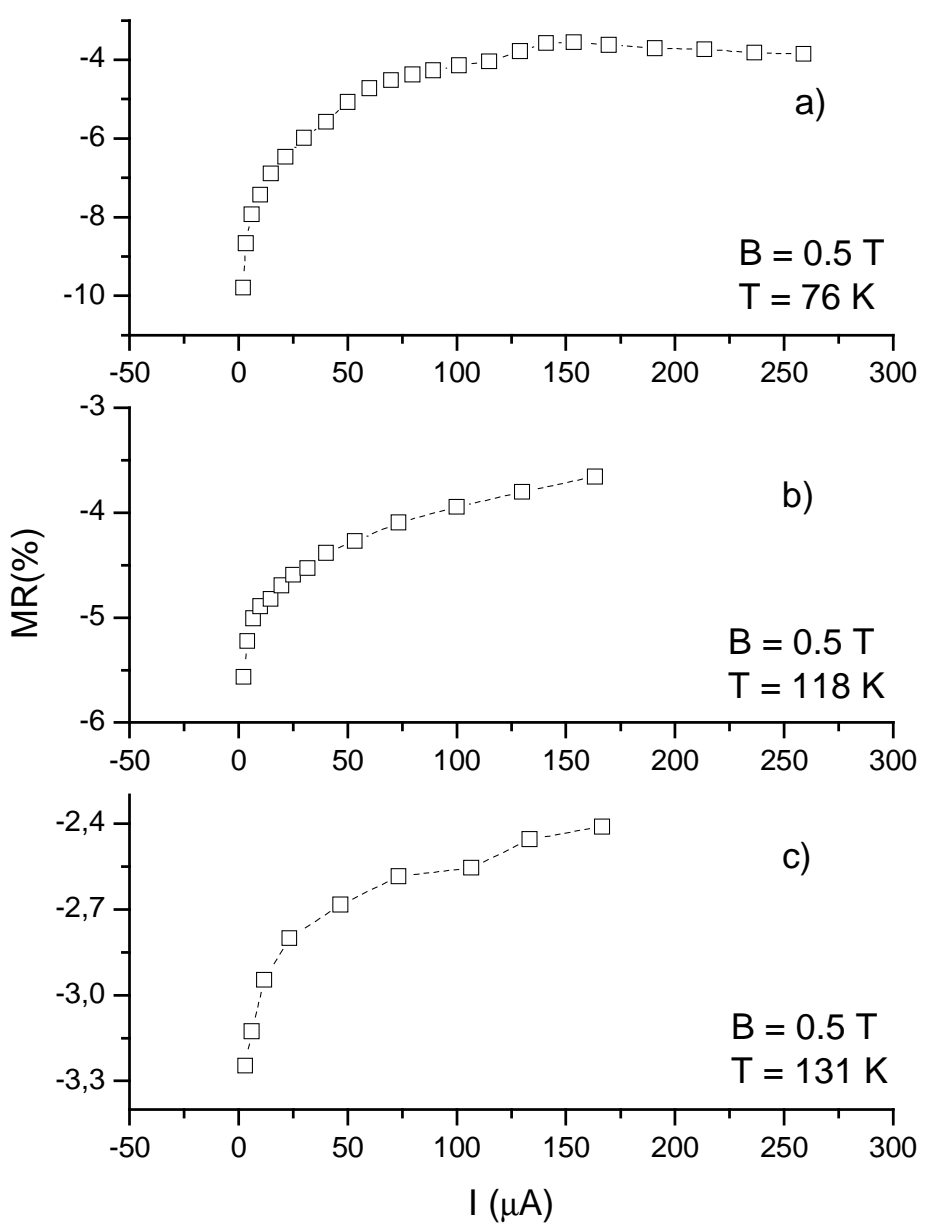

Abbildung 3.27: Die Abhängigkeit des magnetoresitiven Effektes von der Strombelastung unter dem magnetischen Feld $B=0.5 T\left(\mathrm{Nd}_{2 / 3} \mathrm{Sr}_{1 / 3} \mathrm{MnO}_{3-\delta} \delta=0.10\right)$.

Für die Probe mit $\delta=0.15$ sind die unter dem Belastungsstrom $\mathrm{I}=28.5 \mu \mathrm{A}$ gemessenen Widerstandskurven $\rho(\mathrm{T})$ unter verschiedenen magnetischen Feldern (von 0 bis $0.5 \mathrm{~T}$ ) in der Abbildung 3.28 gezeigt. Die Probe zeigt generell den üblichen negativen Magnetowiderstand, der aber noch viel kleiner als bei allen vorherigen Proben ist. Es gibt aber bei dieser Probe eine sprungartige Anomalie, die auch ohne das Magnetfeld zu finden ist. Das Magnetfeld kann die Position dieser Anomalie stark beeinflussen. Die unter dem Feld B $=0$ T gemessene $\rho(T)-K u r v e$ zeigt einen Sprung bei $\mathrm{T}_{\mathrm{K}}=106 \mathrm{~K}$. Bei der Kurve unter $0.25 \mathrm{~T}$ verschiebt sich der $\mathrm{T}_{\mathrm{K}}$-Punkt zu 105 K. Man braucht also tiefere Temperaturen, um den Übergang unter einem Magnetfeld zu induzieren. Die nächsten zwei Kurven (gemessen unter dem Magnetfeld B =0.37 und 0.5 T) zeigen überraschenderweise überhaupt keinen Übergang mehr. Das bedeutet aber, dass man mit dem Magnetfeld die Möglichkeit hat, die gesamte Probe in einem einheitlichen Zustand zu halten. Daraus kann man auch wieder folgern, dass das magnetische Feld dem elektrischen Feld entgegen wirkt. 


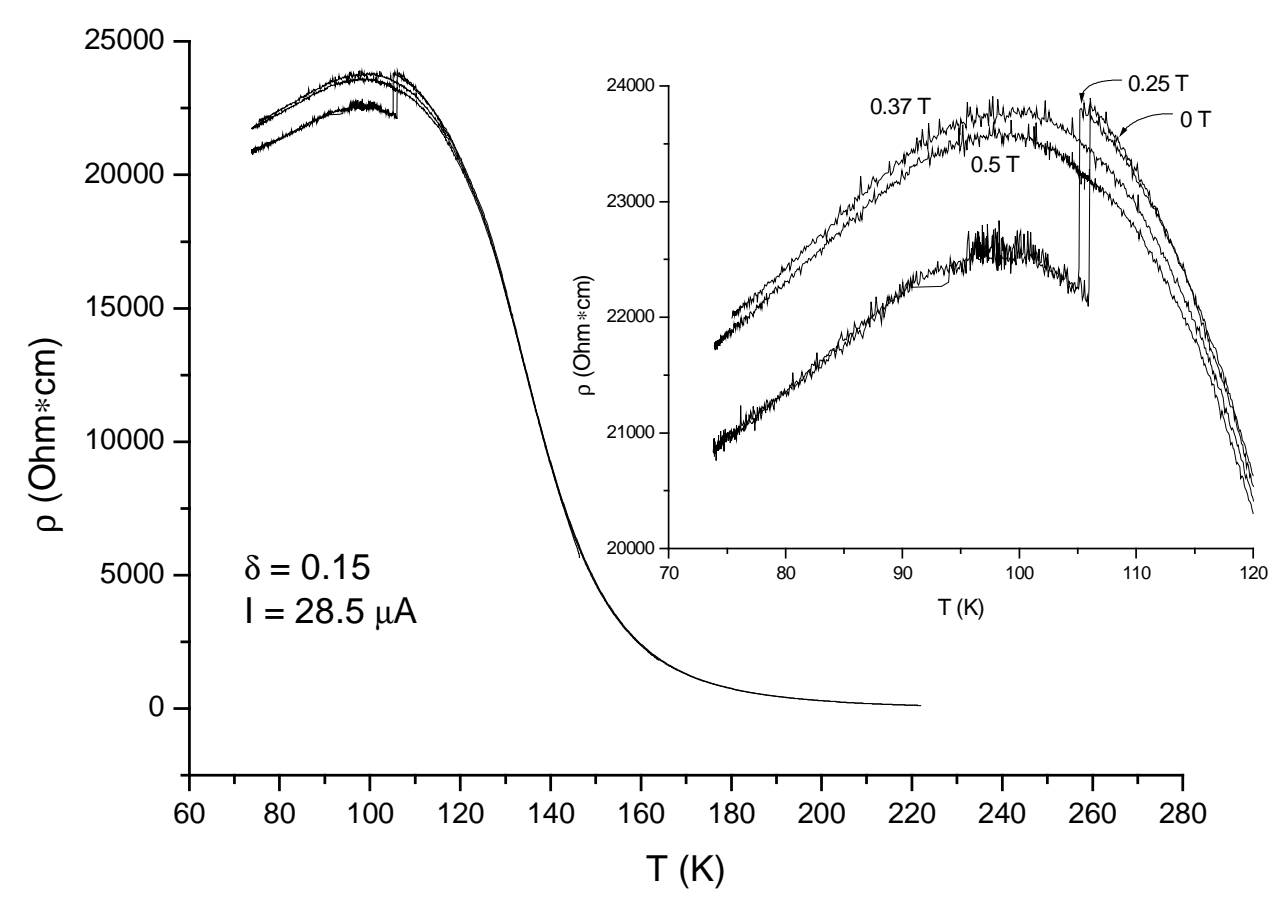

Abbildung 3.28: Der spezifische Widerstand einer $\mathrm{Nd}_{2 / 3} \mathrm{Sr}_{1 / 3} \mathrm{MnO}_{3-\delta^{-}}$Probe mit $\delta=0.15$ unter verschiedenen magnetischen Feldern.

Die B-Probe mit $\delta=0.20$ zeigt ebenfalls eine solche $\mathrm{T}_{\mathrm{K}}$-Anomalie (Abb. 3.29). Diese wird mit dem magnetischen Feld auch ähnlich beeinflusst. Die Tendenz ist hier genau so wie bei der Probe mit $\delta=0.15$ : bei größeren Feldern ist die $\mathrm{T}_{\mathrm{K}}$-Temperatur kleiner. Zusätzlich wurde hier der Widerstand auch während der Aufheizung gemessen (unter dem Magnetfeld $\mathrm{B}=0.15$ und $0.5 \mathrm{~T}$ ). Diese Messungen zeigen, dass es eine Temperaturhysterese gibt, die offenbar mit $\delta$ größer wird. Wegen der nur teilweisen Umwandlung wird vermutet, dass dieser Übergang nahe der Korngrenzen abläuft.

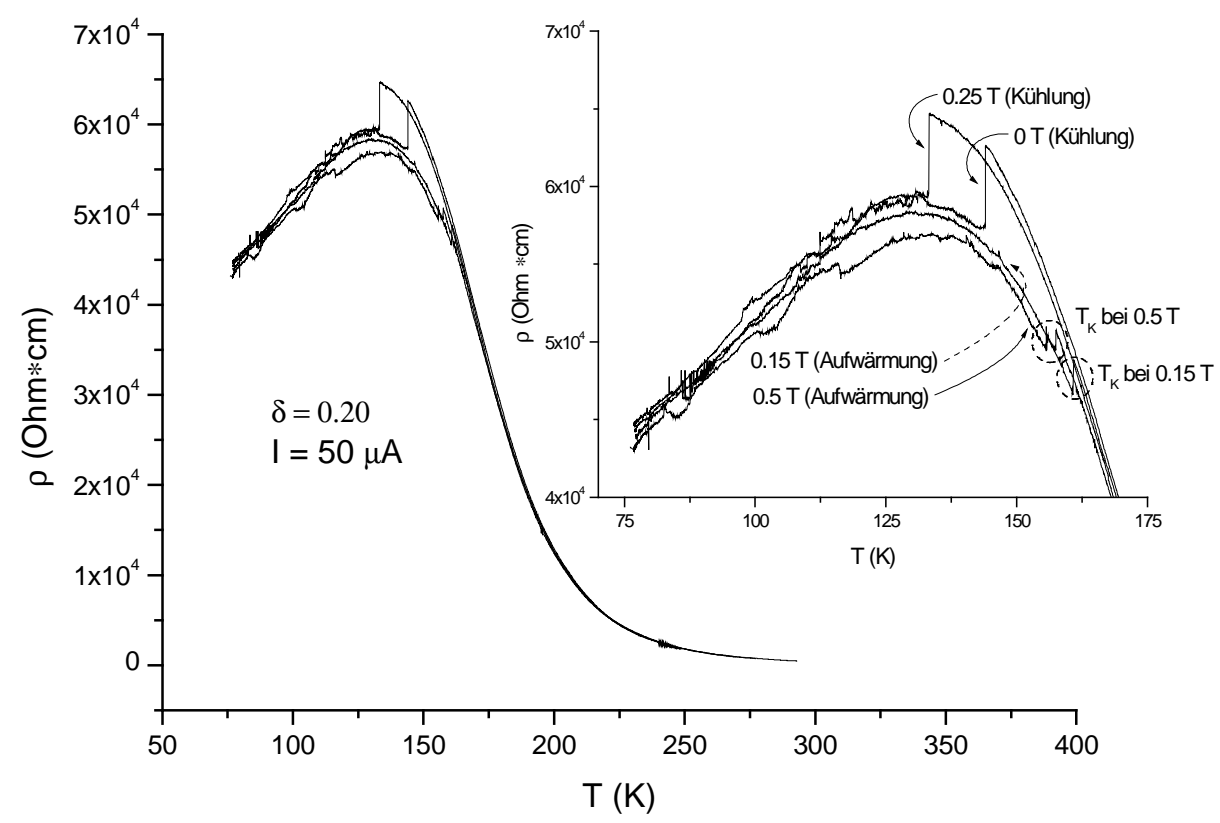

Abbildung 3.29: Der spezifische Widerstand der $N d_{2 / 3} \mathrm{Sr}_{1 / 3} \mathrm{MnO}_{3-\delta}-\mathrm{B}$-Probe $(\delta=0.20)$ unter verschiedenen magnetischen Feldern. 


\subsection{Die Thermokraft $S$}

Die Messungen des durch einen Temperaturgradienten induzierten elektrischen Feldes (Thermokraft, „TEP“) liefern weitere Informationen. Es gibt viele Messungen der Thermokraft an stöchiometrischen Manganiten, die alle den Metall-Isolator-Übergang aufzeigen [17-20]. Wir wollten sehen, ob man auch hier eine Korrelation zwischen den $\rho(\mathrm{T})$-Messungen und der Thermokraft S finden kann. Wir haben die Thermokraft mit Hilfe der TTE-Methode (siehe Kapitel „Experimentelles“) im Bereich von 50 bis $400 \mathrm{~K}$ gemessen. Da die TTE-Methode keine absoluten Thermokräfte bestimmen kann, wurden bei $\delta=0$ konventionell erhaltenen Daten [17] zu Kalibrierung der Thermokraft S benutzt. Diese an der Serie 1 gemessene Thermokraft ist in der Abbildung 3.30 gezeigt.

Die Messungen an den Proben mit kleinem Sauerstoffdefizit $(\delta \leq 0.10)$ sind gut mit Literaturdaten vergleichbar [18]. Die Proben mit $\delta=0$ und 0.05 zeigen einen Null-Durchgang bei $\approx 175$ und 275 K. Für die Probe mit $\delta=0.10$ kann aus der Extrapolation ein Kreuzungspunkt mit der T-Achse bei ungefähr $350 \mathrm{~K}$ vermutet werden. Links vom Durchgangspunkt befindet sich ein Maximum, d.h. die Thermokraft verschwindet wieder bei tieferen Temperaturen. Dieses Maximum verschiebt sich mit der $\delta$-Erhöhung zu den höheren Temperaturen. Allgemein wird bei allen Proben die maximale Thermokraft mit der Erhöhung des Sauerstoffdefizits größer.

Bei kleinen $\delta$ ist eine direkte Korrelation zwischen den Thermokraftmessungen und den Widerstandsmessungen nicht zu finden. Wenn wir allerdings die Messungen an den Proben mit $\delta$ $\geq 0.15$ in Betracht ziehen, finden wir, dass die Thermokraft bei niedrigen Temperaturen $(\mathrm{T}<100$ $\mathrm{K}$ ) dramatisch ansteigt (bis zu $12000 \mu \mathrm{V} / \mathrm{K}$ ). Ein solches Verhalten zeigen normalerweise Manganitproben, die sich im isolierendem Zustand befinden [8]. Wenn wir das mit den Widerstandsmessungen vergleichen (siehe Abb. 3.30), können wir vermuten, dass der Widerstand der Probe mit $\delta=0.15$ bei tieferen Temperaturen weiter ansteigt (gestrichelte Linie in Abbildung 3.30). Genau dasselbe Verhalten finden wir bei der Probe mit $\delta=0.20$. Andererseits sollte man nach den Thermokraftmessungen an der Probe mit $\delta=0.10$ erwarten, dass sie bei tieferen Temperaturen doch noch in den metallischen Zustand übergeht.

\subsection{Die Wärmeleitfähigkeit K}

Die Wärmeleitfähigkeit kann mit Hilfe der TTE-Methode ebenfalls gemessen werden. Auch hier versuchen wir eine Korrelation zwischen den elektrischen Widerstands- und Wärmewiderstandsmessungen zu finden, z.B. aufgrund des Wiedermann-Franz'schen Gesetzes. Dieses Gesetz sagt aus, dass das Verhältnis der thermischen und der elektrischen Leitfähigkeit zur Temperatur direkt proportional ist $(\mathrm{K} / \sigma \sim \mathrm{T})$; dabei ist die Proportionalitätskonstante vom jeweiligen Material unabhängig.

Zunächst zeigt die K-Messung (Abb. 3.31) für die stoichiometrische Substanz mit Literaturdaten [17] eine gute Übereinstimmung. Die Probe mit dem Defizit $\delta=0.20$ zeigt eine ausgeprägte 
Anomalie in der Nähe von $150 \mathrm{~K}$. Ein ähnliches Verhalten ist auch noch bei der Probe mit $\delta=$ $0.15 \mathrm{zu}$ finden, jedoch weniger ausgeprägt. Wir können Spuren dieser Anomalie sogar noch bei der Probe mit $\delta=0.10$ finden, bei kleinen $\delta$ aber nicht. Dies bestätigt wieder die zwei $\delta$-Regime. Thermokraft-, Wärmeleitfähigkeits-, Widerstands- und Magnetisierungsmessungen zusammen bestätigen also, dass bei der $\delta$-Variation zwei verschiedene Regime zu beachten sind. Dabei befindet sich die Probe mit $\delta=0.10$ genau in Übergangsbereich. 

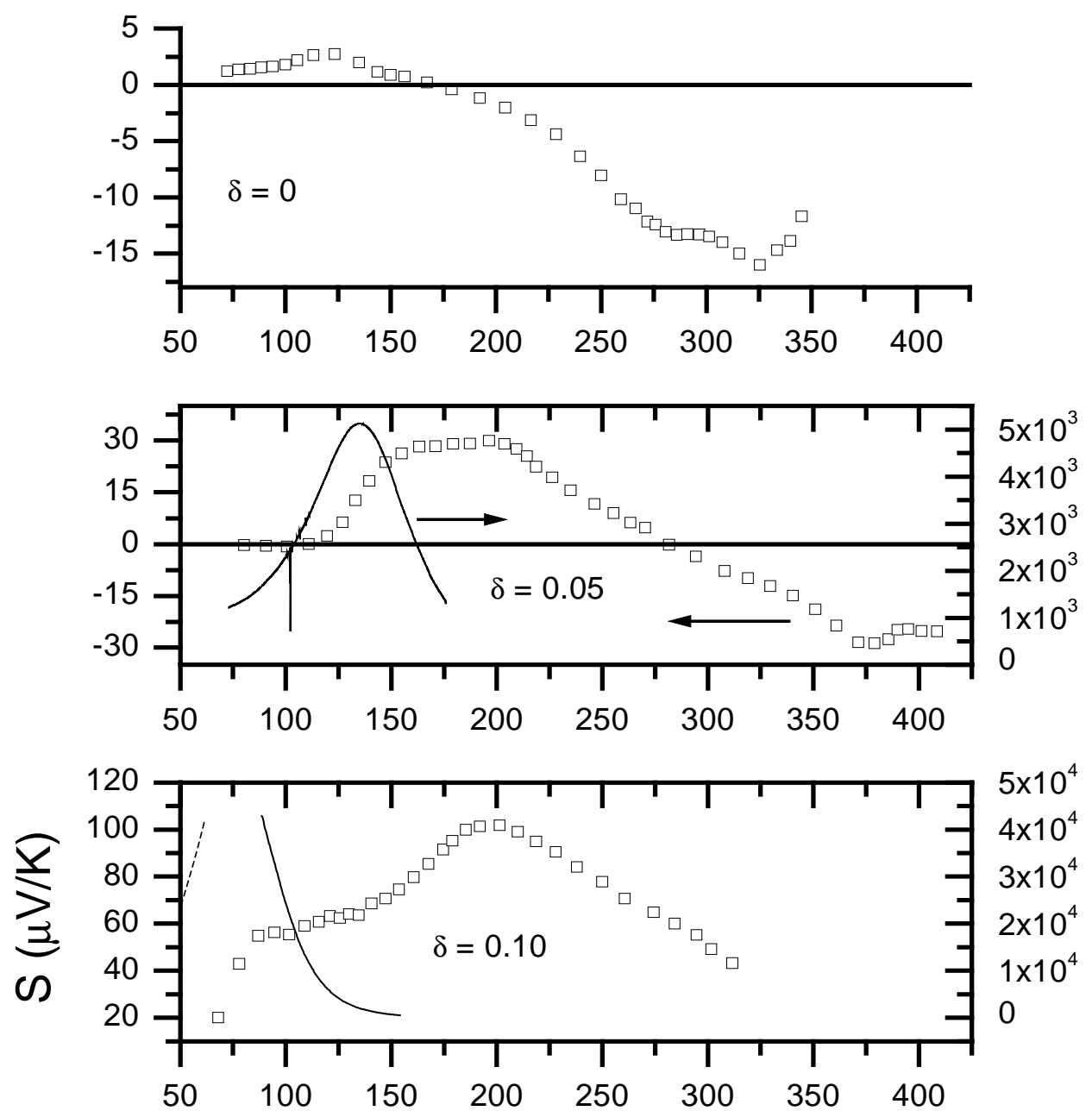

$5 \times 10^{4}$

$4 \times 10^{4}$

$3 \times 10^{4}$

$2 \times 10^{4}$

$1 \times 10^{4}$

0 อ

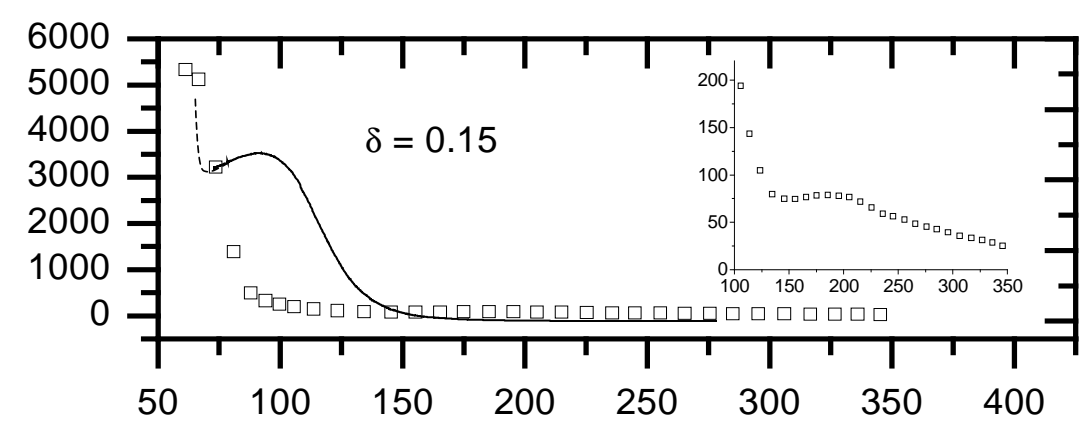

$1,6 \times 10^{5}$

$1,2 \times 10^{5}$

$8,0 \times 10^{4}$

$4,0 \times 10^{4}$

0,0

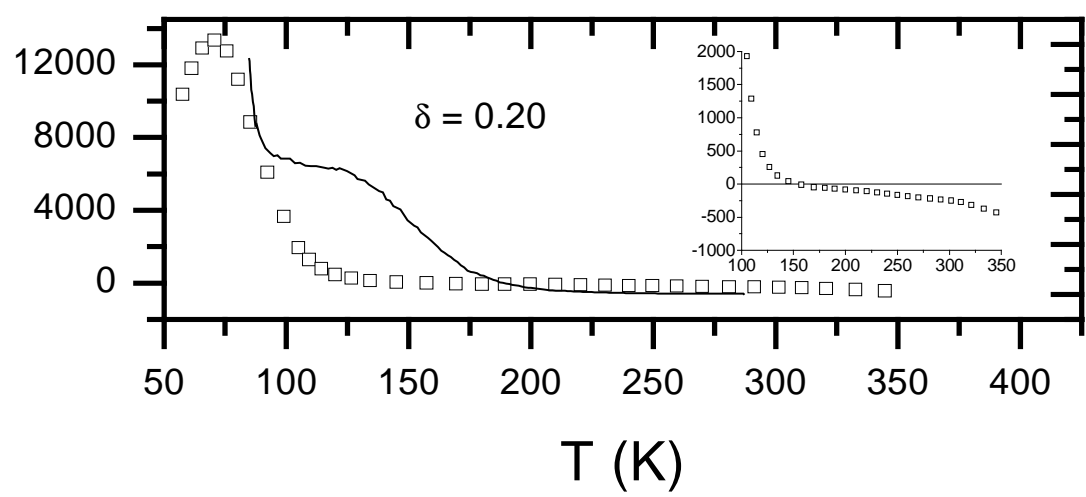

$2,5 \times 10^{5}$

$2,0 \times 10^{5}$

$1,5 \times 10^{5}$

$1,0 \times 10^{5}$

$5,0 \times 10^{4}$

0,0

Abbildung 3.30: Die Temperaturabhängigkeit der Thermokraft von den keramischen $\mathrm{Nd}_{2 / 3} \mathrm{Sr}_{1 / 3} \mathrm{MnO}_{3-\delta}$ Proben (Serie 1). Zusätzlich wurden die Widerstandskurven aufgetragen. 


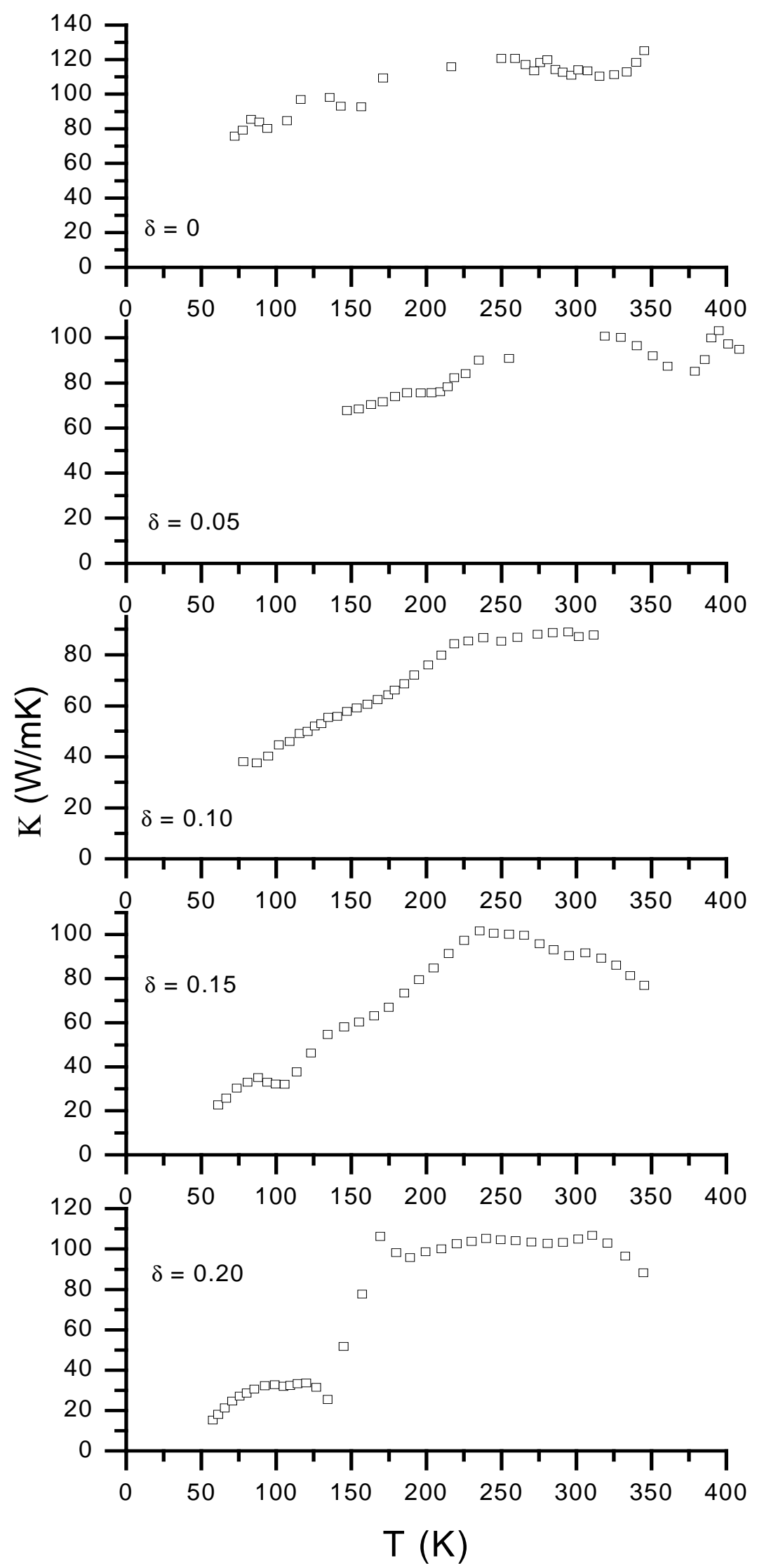

Abbildung 3.31: Die Temperaturabhängigkeit der Wärmeleitfähigkeit von den keramischen $\mathrm{Nd}_{2 / 3} \mathrm{Sr}_{1 / 3} \mathrm{MnO}_{3-\delta}$ Proben (Serie 1). 


\section{Diskussion}

\subsection{Physik der Manganite - die Grundideen.}

\section{Elektronische Struktur in einem Kristallfeld und Jahn-Teller-Effekt.}

In reinem $\mathrm{NdMnO}_{3}$ liegt das Mangan-Ion $\left(\mathrm{Mn}^{+3}\right)$ nominell in der Konfiguration [Ar] $3 \mathrm{~d}^{4}$ vor, in $\mathrm{SrMnO}_{3}\left(\mathrm{Mn}^{+4}\right)$ als $[\mathrm{Ar}] 3 \mathrm{~d}^{3}$. Die elektronische Struktur eines isolierten Mn-Ions entspricht also einer Edelgaskonfiguration und einer teilweise gefüllten 3d-Schale. Ähnliches gilt für andere Übergangsmetalle wie V, Cr, Fe, Co oder Ni. Die fünf Orbitale der 3d-Schale ergeben sich aus den Wasserstofffunktionen, und haben im Falle eines freien Ions alle die gleiche Energie, es wird von entarteten Energieniveaus gesprochen. Wenn wir das Auffüllen der Orbitale diskutieren, müssen wir noch die erste Hundsche Regel berücksichtigen: Um die Coulomb-Abstoßung zu minimieren, bilden die 3d-Elektronen einen Zustand mit maximal möglichem Spin. Dies entspricht einer symmetrischen Spinfunktion. Da die Gesamtwellenfunktion antisymmetrisch sein muss, muss die Ortsfunktion antisymmetrisch sein, denn dann ist die Coulomb-Abstoßung minimal. Anschaulich gesagt gehen sich die Elektronen im Ortsraum so gut wie möglich aus dem Weg, um ihre gegenseitige Abstoßung zu minimieren. Dies ist auch noch so in Festkörper mit Kristallfeld.
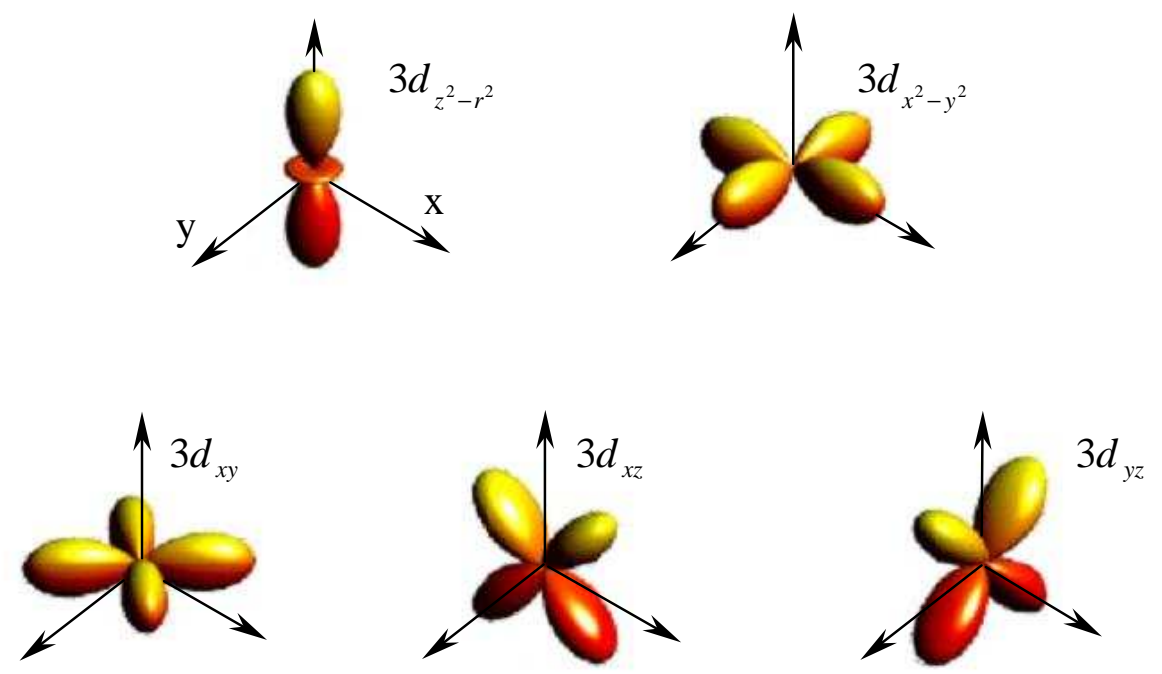

Abbildung 4.1: Die fünf Orbitale der d-Schale im kubischen Kristallfeld. Sie werden in zwei $e_{g^{-}}$ Orbitale (oben) und drei $t_{2 g}$-Orbitale (unten) eingeteilt. Dabei zeigen die $e_{g}$-Orbitale in Richtung der Kristallachsen $x, y, z$ und die $t_{2 g}$-Orbitale entlang der Diagonalen.

In einem Festkörper haben wir es nicht mit freien Ionen zu tun. Betrachten wir zuerst undotiertes $\mathrm{NdMnO}_{3}$. Da jedes $\mathrm{Mn}^{+3}$-Ion in diesem System von einem $\mathrm{O}^{-2}$-Oktaeder umgeben ist (siehe Abbildung 4.2a), sind die fünf d-Orbitale nicht mehr alle energetisch gleich. Die Aufhebung der Entartung ist hier im Wesentlichen durch die Coulomb-Abstoßung der $\mathrm{O}^{-2}$-Ionen des SauerstoffOktaeders bestimmt, der das Mn-Ion umgibt. Man sagt auch: das Kristallfeld, dass sich durch die $\mathrm{O}^{-2}$-Ionen ergibt, hebt die Entartung der 3d-Orbitale auf. Die $\mathrm{O}^{-2}$-Ionen befinden sich auf den 
Koordinatenachsen (x, y, z). Ein Elektron des Mangan-Ions in einem d-Orbital, das auf ein $\mathrm{O}^{-2}$ Ion zeigt, hat wegen der elektrostatischen Abstoßung eine höhere Energie als ein Elektron in einem Orbital, das zwischen den Ionen ausgerichtet ist. Demnach haben die auf den Sauerstoff weisenden Orbitale $d_{x^{2}-y^{2}}$ und $d_{z^{2}}{ }^{8}$ eine höhere Energie. Sie sind untereinander in oktaedrischer Umgebung energetisch äquivalent und werden als $\mathrm{e}_{\mathrm{g}}$-Orbitale bezeichnet $(\mathrm{e}=$ entartet). Die Elektronen der Orbitale $\mathrm{d}_{\mathrm{xy}}, \mathrm{d}_{\mathrm{xz}}$ und $\mathrm{d}_{\mathrm{yz}}$ weichen den $\mathrm{p}$-Orbitalen des $\mathrm{O}^{-2}$-Ions aus und liegen daher energetisch tiefer. Sie sind auch in oktaedrischer Umgebung untereinander äquivalent und werden als $\mathrm{t}_{2 \mathrm{~g}}$-Orbitale bezeichnet $(\mathrm{t}=$ dreifach (triple) entartet).
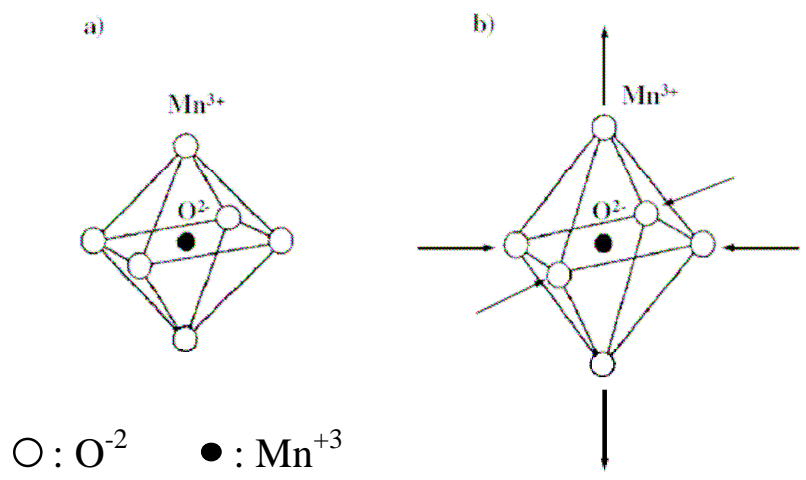

Abbildung 4.2: Verzerrung des Sauerstoffoktaeders durch den Jahn-Teller-Effekt.

Misst man experimentell die genauen Positionen der Sauerstoffionen im $\mathrm{NdMnO}_{3}-\mathrm{Kristall}_{\text {aus, }}$ so stellt man fest, dass der Oktaeder verzerrt ist, so dass vier Sauerstoffionen dem $\mathrm{Mn}^{+3}$ näher und zwei weiter weg stehen als im unverzerrten Fall (Abbildung 4.2b). Wie kommt es zu dieser Gitterverzerrung? Bei der Besetzung der d-Orbitale mit Elektronen werden in oktaedrischer Umgebung zuerst die $\mathrm{t}_{2 \mathrm{~g}}$-Orbitale und anschließend die $\mathrm{e}_{\mathrm{g}}$-Orbitale gemäß der ersten Hundschen Regel aufgefüllt. Die ersten drei d-Elektronen eines $\mathrm{Mn}^{+3}$-Ions besetzen die drei $\mathrm{t}_{2 \mathrm{~g}}$-Orbitale. Das vierte d-Elektron besetzt eines der beiden $e_{\mathrm{g}}$-Orbitale. Da diese Orbitale auch in oktaedrischer Umgebung die gleiche Energie haben, ist von vornherein nicht eindeutig festgelegt, welches von ihnen vom vierten Elektron besetzt wird. Es liegt eine orbitale Entartung vor. Es zeigt sich, dass ein Kristall mit einer solchen orbitalen Entartung instabil ist gegenüber Verzerrungen des Kristallgitters, die diese Entartung aufheben. Dies folgt direkt aus dem Jahn-Teller Theorem [62, 63]. Diesen Effekt kann man aus folgenden einfachen Überlegungen leicht verstehen. Nehmen wir an, dass der Oktaeder in z-Richtung verzerrt ist. Es ist sofort klar, dass von den beiden $\mathrm{e}_{\mathrm{g}}{ }^{-}$ Orbitale des $\mathrm{Mn}^{+3}$-Ions das $3 d_{z^{2}}$-Orbital nun energetisch begünstigt ist, da sich die Punktladungen der negativen Sauerstoffionen vom $\mathrm{Mn}^{3+}$-Ion entfernt haben. Umgekehrtes gilt für das $3 d_{x^{2}-y^{2}}$-Orbital: Die Sauerstoffionen sind aufgrund der Verzerrung etwas herangerückt, was einer Erhöhung des Energieeigenwerts zur Folge hat. Damit ist die zweifache Entartung der

\footnotetext{
${ }^{8}$ In der Literatur wird dieses Orbital oft einfachheithalber als $d_{z^{2}}$ beteichnet. Die genauere Bezeichnung ist aber $d_{z^{2}-r^{2}}$.
} 
$\mathrm{e}_{\mathrm{g}}$-Niveaus aufgehoben. Wie man in der Abbildung 4.3 sieht, sind auch die drei $\mathrm{t}_{2 \mathrm{~g}}$-Orbitale betroffen. Der $x y$-Zustand hat eine höhere Energie, da die umgebenden Sauerstoffionen herangerückt sind. Umgekehrt haben die beiden Orbitale mit $z$-Anteilen energetisch eine Absenkung erfahren. Da die fünf Orbitale der 3d-Schale in $\mathrm{Mn}^{+3}$ mit nur vier Elektronen besetzt sind, verbessert sich durch die Gitterverzerrung insgesamt die Energiebilanz, weil das oberste $\left(x^{2}\right.$ $-y^{2}$ )-Orbital unbesetzt bleibt. Da das $\mathrm{Mn}^{+4}$-Ion nur drei Elektronen hat (das $\mathrm{z}^{2}$-Orbital bleibt unbesetzt), bringt eine Verzerrung des Oktaeders keinen Energiegewinn, da das $\mathrm{t}_{2 \mathrm{~g}}$-Zentrum erhalten bleibt und findet deshalb auch gar nicht statt.

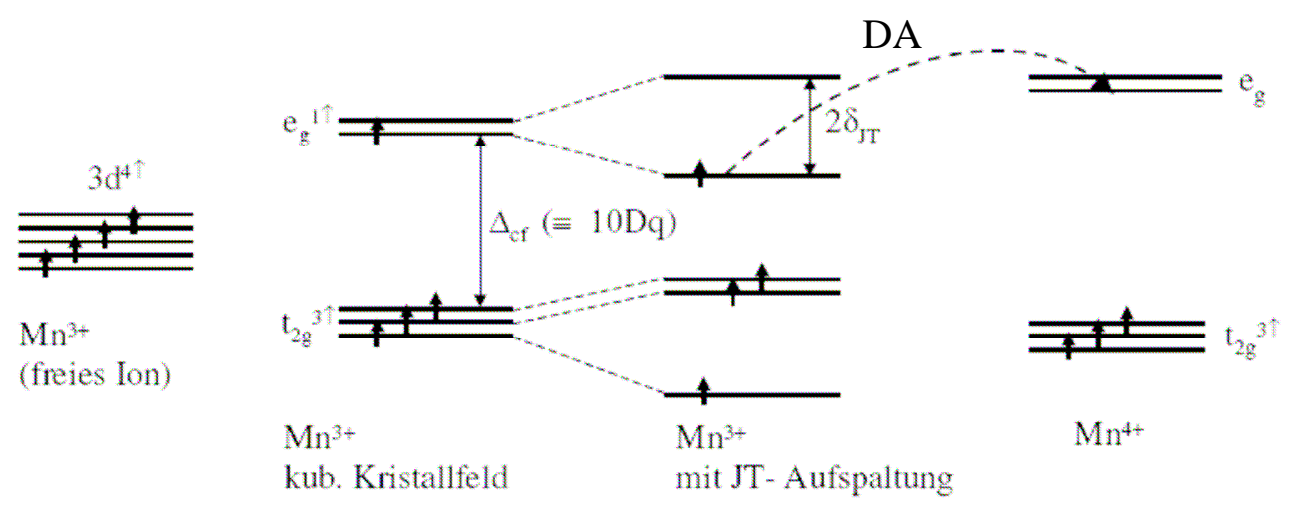

Abbildung 4.3: Elektronenzustände des $M n^{+3}$ - und des $M n^{+4}$-Ions. Dq bezeichnet die Kristallfeldaufspaltung und $2 \delta_{J T}$ die Jahn-Teller-Aufspaltung. DE deutet den Elektronentransfer beim ferromagnetischen Doppelaustausch (DA) an.

Kristallfeld- und Jahn-Teller-Theorem sind sicher bei der Lokalisierung des Zenerelektrons anwendbar. Bei der Bewegung des Zenerelektrons sind die elektronischen und phononischen Zustände anders.

\section{„Doppelaustauch“-Mechanismus (DA) und Polaronenmodell}

Die meisten Eigenschaften der Manganite sind mit dem sogenannten „Doppelaustauch“Mechanismus (,double-exchange“) eng verbunden. Dieses Phänomen hat Zener schon im Jahr 1951 beschrieben [27]. Der Doppelaustauschmechanismus ist in der Abbildung 4.4 schematisch dargestellt.

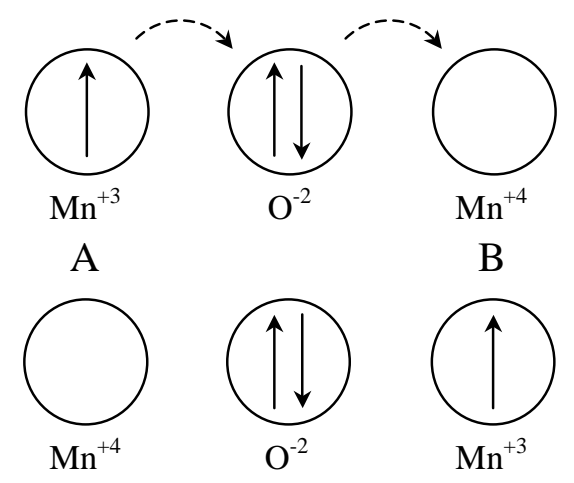

Abbildung 4.4: Schematische Darstellung des Doppelaustauschmechanismus. Nach [50] 
Bei diesem Prozess bewegen sich gleichzeitig zwei Elektronen. Diese Bewegungen kann man schematisch wie folgt beschreiben: $\mathrm{Mn}_{1 \uparrow}^{+3} \mathrm{O}_{2 \uparrow, 3 \downarrow} M n^{+4} \leftrightarrow M n^{+4} O_{1 \uparrow, 3 \downarrow} M n_{2 \uparrow}^{+3}$. Das Elektron 1 des linken Mn-Ions geht zu dem $\mathrm{O}^{-2}$-Ion über bei gleichzeitigem Übergang des Elektrons 2 vom $\mathrm{O}^{-2}$ zu dem rechten Mn-Ion. Dabei werden die Spinmomente der beiden Mn-Ionen dank der Hund'schen Wechselwirkung parallel ausgerichtet. Die Wahrscheinlichkeit eines solchen Elektronenaustausches hängt selbstverständlich auch von der Temperatur ab, da der Ausrichtung der Spinmomente die entropische Kraft entgegensteht. Dies erklärt auch die übliche Form der $\rho(\mathrm{T})$-Kurve (siehe z.B. Abb. 4.11 ). Bei tiefen Temperaturen bis zum $\mathrm{T}_{\mathrm{C}}$-Punkt wird metallische Leitfähigkeit beobachtet. Die momentane Wahrscheinlichkeit des Doppelaustauschprozesses zwischen zwei benachbarten $\mathrm{Mn}^{+3}$ und $\mathrm{Mn}^{+4}$ Ionen bei der Temperatur $\mathrm{T}=0 \mathrm{~K}$ wird dann geschrieben [35]:

$\mathrm{t}_{\mathrm{eff}}=\mathrm{t}_{0} \cos (\theta / 2)$

$\theta$ ist hier der Winkel zwischen den Spinrichtungen der zwei Mn-Ionen und $t_{0}$ ist die Wahrscheinlichkeit des Elektronenüberganges zwischen Mn-Ionen, deren Spins parallel ausgerichtet sind. Bei den Temperaturen $\mathrm{T}>0$ muss man den thermischen Mittelwert für die fluktuierenden Spins $\langle\cos (\theta / 2)\rangle$ benutzen. Bei Temperaturabsenkung erhöht sich die Spinordnung, d.h. $\theta$ wird generell kleiner. Das bedeutet aber eine Erhöhung der Leitfähigkeit. Bei Temperaturen oberhalb des $\mathrm{T}_{\mathrm{C}}$-Punktes verschwindet die ferromagnetische Ordnung der MnIonen $(<\cos (\theta / 2)\rangle=0)$ und die Leitfähigkeit wird geringer (Isolator). Das aktivierte Verhalten der Leitfähigkeit dieses Zustandes kann nicht allein im Rahmen der einfachen Doppelaustauschtheorie erklärt werden. Um ein solches Verhalten zu beschreiben, wurden zusätzlich Ideen über die Lokalisierung der Ladungsträger (für $\mathrm{T}>\mathrm{T}_{\mathrm{mi}}$ ) und über die Ausbildung von Polaronen entwickelt. Heutzutage gibt es aber noch keine einheitliche Theorie. In der aktuellen Literatur sind ebenfalls verschiedene Ansätze zur Beschreibung des Widerstandsverhaltens im Hochtemperaturbereich zu finden:

$\rho=\rho_{0} \exp (E / k T) \quad$ - aktivierte Resistivität von Halbleitern (,semiconductor behaviour“) [67, 68, 69];

$\rho \sim \operatorname{Texp}\left(E_{a} / k T\right) \quad$ - Hüpfleitung über kleine Polaronen (,small polaron hopping“) [70, 74, 75, 76];

$\rho=\rho_{0} \exp \left(T_{0} / T\right)^{-1 / 4}-$ Hüpfleitung über statistische Potentiale unterschiedlicher Breite (,variable range hopping") $[77,78,79,80]$.

Die Abbildung 4.5 zeigt den spezifischen Widerstand der keramischen $\mathrm{Nd}_{2 / 3} \mathrm{Sr}_{1 / 3} \mathrm{MnO}_{3}$-Probe oberhalb $\mathrm{T}_{\mathrm{C}}$ als Funktion $\ln \left(\rho\left(T^{-1}\right)\right)$. Angepasst ist die analytische Funktion $\rho=\rho_{0} \exp \left(E_{0} / k T\right)$ auf den Temperaturbereich oberhalb $\mathrm{T}_{\mathrm{mi}}$. Die beste Anpassung (Fit) ergibt eine Energielücke von $\mathrm{E}_{0}$ $=0.11 \mathrm{eV}$. Man sieht dabei, dass die thermisch aktivierte Beschreibung nur oberhalb $259 \mathrm{~K}$ anwendbar ist. Unterhalb dieser Temperatur wird die kohärente Elektronenbewegung bemerkbar. 


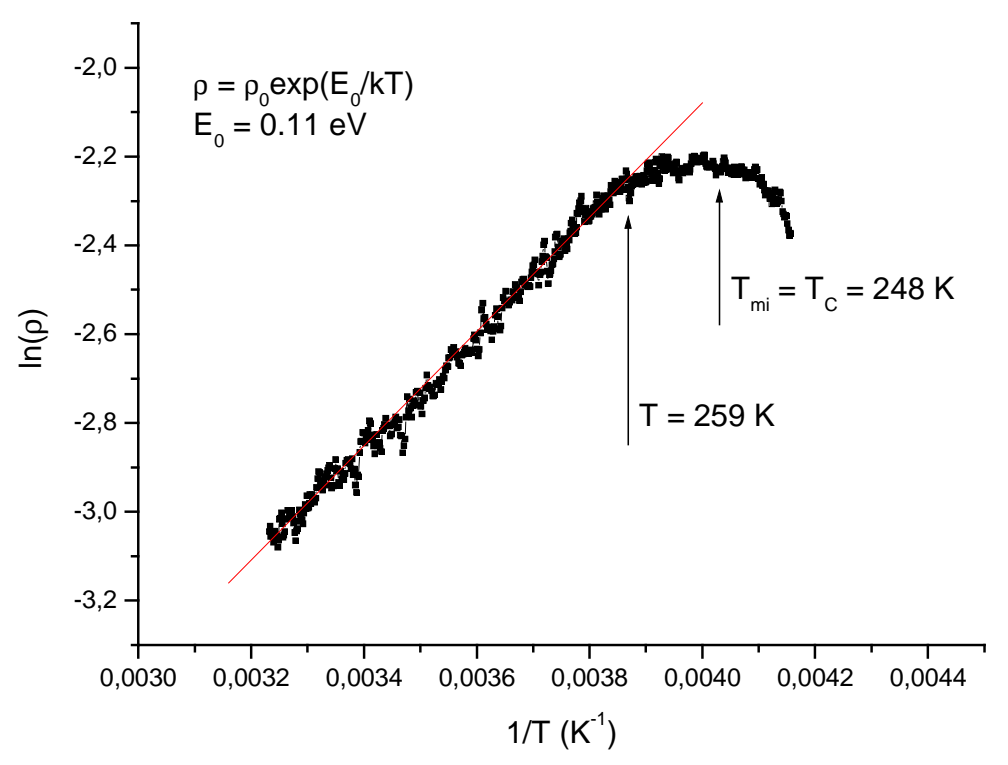

Abbildung 4.5: Anpassung des thermisch aktivierten Teils der Resistivitätskurve einer keramischen $\mathrm{Nd}_{2 / 3} \mathrm{Sr}_{1 / 3} \mathrm{MnO}_{3}$-Probe.

\section{Goodenough's Bedingung für die „Zener bond“-Blockierung}

Die elektrischen und magnetischen Eigenschaften sind also in Manganiten sehr eng verbunden. Dies liegt an dem Doppelaustauschmechanismus. Der DA-Prozess kommt aber zwischen benachbarten $\mathrm{Mn}^{+3}$ - und $\mathrm{Mn}^{+4}$-Ionen nicht immer zustande. Goodenough hat eine zusätzliche Bedingung für den Ladungstransfer zwischen den $\mathrm{Mn}^{+3}$ und $\mathrm{Mn}^{+4}$ Ionen formuliert [48]. Damit das mit den Sauerstoffoktaedern umgebene $\mathrm{Mn}^{+3} / \mathrm{Mn}^{+4}-\mathrm{Paar} \mathrm{zu}$ einem DA-Resonanzzustand kommen kann, muss dieses Paar von Oktaedern umgeben sein, die nur $\mathrm{Mn}^{+3}$-Ionen enthalten. Diese Bedingung erklärt sofort, warum die Manganitsysteme das höchste magnetische Moment und die höchste Curie-Temperatur in der Nähe von $\mathrm{x} \approx 0.3$ zeigen (nicht bei $\mathrm{x}=0.5$, wie beim Anderson-Hasegawa-Modell, siehe S.50). Dies erklärt auch, warum im elektrondotierten Bereich, $\mathrm{x}>0.5$, keine ferromagnetische Phase existiert - die Goodenough-Bedingung ist hier einfach nicht erfüllt. Im elektrondotierten Bereich gibt es statistisch gesehen ganz selten eine $\mathrm{Mn}^{+4}-\mathrm{Mn}^{+3}$-Paar als Zentrum einer $\mathrm{Mn}^{+3} \mathrm{O}^{-6}$-Nahumgebung. In der Literatur wird diese Bedingung mit der Polarontheorie in Verbindung gebracht [54]. Es ist bekannt, dass das $\mathrm{Mn}^{+3}$ Ion im Kristallfeld ein Jahn-Teller-Ion ist, aber $\mathrm{Mn}^{+4}$ nicht. Ohne die passende Nahumgebung gibt es keine Möglichkeit die Jahn-Teller-Verzerrung von A nach B (Abb. 4.4) zu übertragen.

\section{Phasenseparation oder kohärent gekantete Phasen?}

In der Abbildung 4.6 ist ein Phasendiagramm gezeigt, dass auch für das Manganitsystem gelten soll. Die paramagnetischen, ferromagnetischen (metallischen) und antiferromagnetischen (isolierenden) Bereiche (pm, fm und afm) werden in der Literatur mehr oder weniger einheitlich beschrieben. Über den Zustand in dem mit dem Fragezeichen markierten Bereich gibt es zur Zeit noch keine einheitliche Meinung. Als Folge der Konkurrenz beider Wechselwirkungen wird in der Literatur kontrovers die Ausbildung einer metallischen gekanteten antiferromagnetischen Phase oder die Separation in ferro- und antiferromagnetische Bereiche diskutiert. 


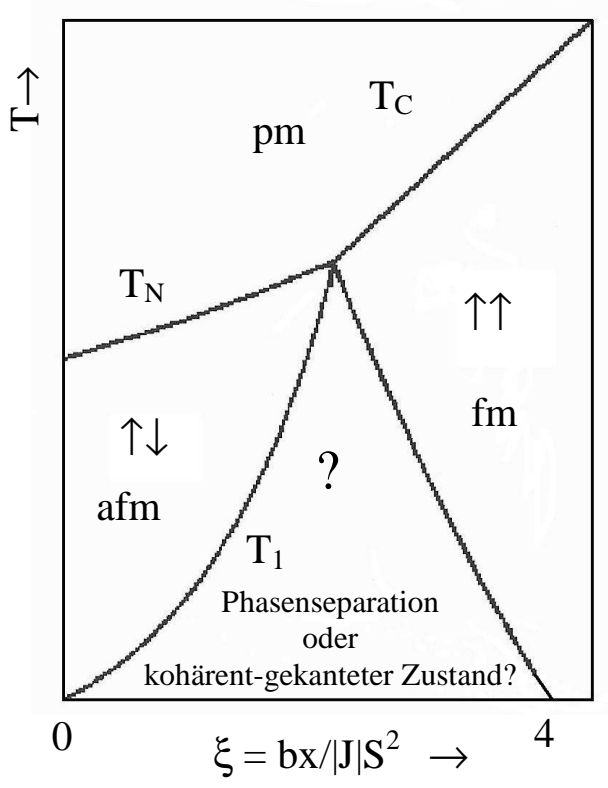

Abbildung 4.6: Das magnetische T- $\xi$ Diagramm nach [23]. $\xi$ ist die sogenannte strukturchemische Variable), die der Zener-Ladungkonzentration x proportional ist $(x \approx 0.15$ bei $\xi=4)$.

\section{Superaustausch}

In den Manganiten, und allgemein in den Übergangsmetalloxiden, werden die magnetischen Eigenschaften im Wesentlichen durch zwei konkurrierenden Mechanismen bestimmt, durch den Superaustausch (SA, ,superexchange“) und durch den Doppelaustausch zwischen den Mn-Ionen. Bei dem Superaustausch entsteht die Wechselwirkung zwischen den beiden Mn-Ionen durch den d-d-Überlapp über die p-Elektronen des Sauerstoff-Iones. Dabei kann der Superaustausch sowohl eine antiferromagnetische als auch eine ferromagnetische Ordnung begünstigen, abhängig von der geometrischen Konfiguration und der Besetzung der 3d-Orbitalen. Goodenough, Kanamori und Anderson haben einfache Regeln formuliert, mit denen Vorzeichen und Betrag der magnetischen Austauschwechselwirkung (SA) in den meisten Fällen richtig vorhergesagt werden kann (siehe z.Bsp. [55]). Die positive oder negative Kopplung hängt in diesem Fall von der Überlappung der leeren bzw. gefüllten $\mathrm{e}_{\mathrm{g}}$-Orbitalen mit dem p-Orbital des Sauerstoffes ab. Generell gilt: wenn an den beiden Seiten des $\mathrm{O}^{-2}$-Iones das p-Orbital mit gefüllten oder leeren $\mathrm{e}_{\mathrm{g}}$-Orbitalen überlappt, gibt es eine antiferromagnetische Ordnung. Wenn es an einer Seite eine Überlappung mit einem gefüllten und an der anderen Seite mit einem leeren Orbital gibt, entsteht eine ferromagnetische Kopplung. Der Doppel-Austausch ist im Gegensatz dazu ausschließlich für eine ferromagnetische Kopplung verantwortlich.

\section{Kohärent gekanteter Zustand.}

In diesem Abschnitt wird die Ausbildung eines kohärent-gekanteten Zustandes beschrieben - als ein mögliches Szenario. Die Theorie dafür hat deGennes noch im Jahr 1960 entwickelt [24]. In einer Konfigurationen, in denen die Spins innerhalb einer Ebene parallel ausgerichtet, aber in je zwei Nachbarebenen um ein Winkel $\theta$ gekantet sind, stellt sich ein Kantungswinkel $\theta_{0}$ ein, der sich aus der Minimierung der Summe der Energien von Super- und Doppelaustausch berechnen lässt. Für die SA-Energie gilt: 


$$
E_{S E}=-N z^{\prime} J^{\prime} S^{2}+N z|J| S^{2} \cos \left(\Theta_{0}\right)
$$

wobei $\mathrm{J}$ und $\mathrm{J}$ ' die antiferro- und ferromagnetische Austauschintegrale ( $\mathrm{J}<0$ und $\mathrm{J}$ ' > 0) sind. N ist hier die Anzahl magnetischer Ionen pro Zellvolumen. $\mathrm{z}$ und $\mathrm{z}$ ' sind die Anzahl der antiferromagnetisch und ferromagnetisch angekoppelten Nachbar-Ionen (Mn).

Zur Berechnung der DA-Energie wird zunächst die Energie $E_{D A, k}$ eines Zener-Ladungsträgers mit dem Wellenvektor $\vec{k}$ benötigt. Die Amplitude der zugehörigen Wellenfunktionen $\Psi_{i}$ ist $\alpha_{i}=e^{i \vec{k} \vec{R}_{i}}$, woraus sich zusammen mit $\Psi=\sum \alpha_{i} \varphi_{i}$ und $t_{i j}=\left\langle\varphi_{i}|H| \varphi_{j}\right\rangle=b_{i j} \cos \left(\Theta_{i j} / 2\right)$ ergibt $^{9}$ :

$E_{D E, k}=\sum_{i, j} t_{i, j} e^{i k\left(R_{j}-R_{i}\right)}=-b^{\prime} \gamma_{k}{ }^{\prime}-b \gamma_{k} \cos \left(\Theta_{0} / 2\right)$

dabei sind $\gamma_{k}{ }^{\prime}=\sum_{j^{\prime}} e^{i \vec{k}\left(\vec{R}_{j^{\prime}}-\vec{R}_{i}\right)}$ und $\gamma_{k}=\sum_{j} e^{i \vec{k}\left(\vec{R}_{j}-\vec{R}_{i}\right)}$ die Summen über die nächsten Nachbarn am Platz $i$ (Phasensưmmen). Unter der Annahme, dass sowohl $b$ als auch $b^{\prime}$ positiv sind, ist $E_{D E, k}$ damit durch

$$
E_{\min }=-b^{\prime} \gamma_{0}{ }^{\prime}-b \gamma_{0} \cos \left(\Theta_{0} / 2\right)
$$

nach unten beschränkt. Die Nx Zener-Ladungsträger besetzen Energien nahe $E_{\min }$, woraus sich die gasammte DA-Energie zu

$$
E_{D E}=N x E_{\min }
$$

ergibt (bei der Annahme, dass x $\ll<1$ ist). Aus der Minimierung der Summe von (4.2) und (4.5) hinsichtlich $\theta$ erhält man:

$$
\cos \left(\frac{\Theta_{0}}{2}\right)=\frac{b x}{4|J| S^{2}}
$$

Daraus sieht man, dass mit steigender Dotierung die für $\mathrm{x}=0$ vorhandene antiparallele Spinausrichtung zunehmend verkantet wird. Für

$$
x>x_{C}=\frac{4|J| S^{2}}{b}
$$

erhalten wir schließlich eine ferromagnetische Spinausrichtung.

\footnotetext{
${ }^{9} \varphi_{\mathrm{i}}$ ist ein volständiger Satz orthogonaler Funktionen, aus denen sich die Wellenfunktionen $\Psi$ linear kombinieren lassen; $\mathrm{t}_{\mathrm{ij}}$ ist dabei das Transfer-Integral zwischen den Ionen $\mathrm{i}$ und $\mathrm{j}$
} 
Kagan, Khomskii und Mostovoy [59] haben auf einen besondere Aspekt der eben durchgeführten klassischen Beschreibung hingewiesen. Der Grundzustand des räumlich homogenen verkanteten Zustandes ist instabil gegenüber einer Phasenseparation. D.h. ohne eine besondere Stabilisierung der Kantung ist $\mathrm{zu}$ erwarten, dass sich z.B. ferromagnetische metallische Cluster in einer antiferromagnetischer Matrix bilden [60, 61].

\section{Lokaler Doppelaustausch und Lokale Spinkantung.}

Nehmen wir nun ein Bravais-Gitter magnetischer Ionen an, dessen Spin-Ordnung im ungestörten System ferromagnetische Schichten antiferromagnetischer Kopplung ausbildet (eine antiferromagnetische Anordnung des A-Typs, siehe Abbildung 4.8a). Dies ist der Fall z.B. in undotiertem $\mathrm{LaMnO}_{3}$ oder $\mathrm{NdMnO}_{3}$. Hier richten sich alle Spins parallel oder antiparallel der bAchse (in der Pbnm-Raumgruppe) aus [83, 84]. Die Substitution eines Lanthanoid-Iones (La oder Nd) mit einem Alkali-Ion (Ca oder Sr) führt zur Valenzänderung eines Mn-Iones $\left(\mathrm{Mn}^{+3} \rightarrow\right.$ $\mathrm{Mn}^{+4}$ ) (Implementierung eines Loches). Aus dem Phasendiagramm (Abbildung 4.7) ist zu sehen, dass die meisten Manganitsysteme bei kleinen Dotierungen gut isolierend sind. Dies bedeutet, dass die implementierten Löcher bei diesen Dotierungswerten lokalisiert bleiben, obwohl Goodenough's Bedingung erfüllt ist.
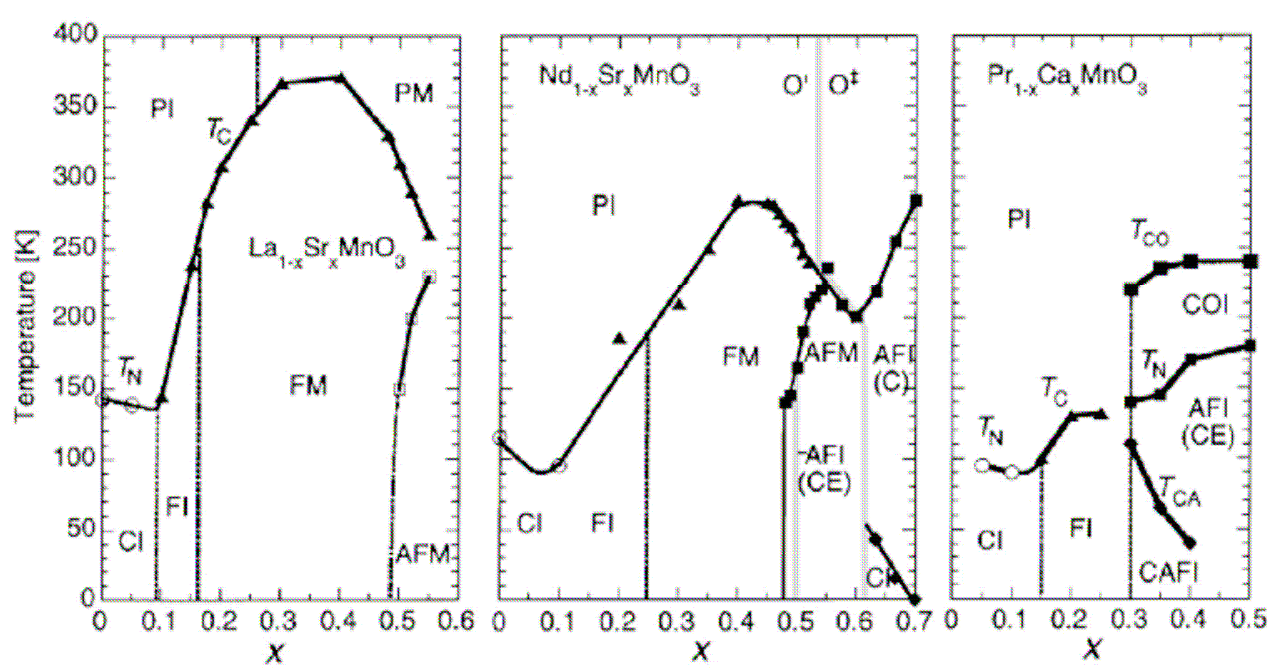

Abbildung 4.7: Phasendiagramme für $\mathrm{La}_{1-x} \mathrm{Sr}_{x} \mathrm{MnO} \mathrm{O}_{3}, \mathrm{Nd}_{1-x} \mathrm{Sr}_{x} \mathrm{MnO}_{3}, \mathrm{Pr}_{1-x} \mathrm{Ca}_{x} \mathrm{MnO}_{3}$ mit folgenden Abkürzungen P: Paramagnetisch, F: Ferromagnetisch, AF: Antiferromagnetisch, C: verkippte Spinordnung, I: Isolator, M: Metall, CO: Ladungsordnung. Nach [58]).

Um dieses Phänomen zu erklären, reicht die Goodenough-Bedingung nicht aus. Es muss die Wirkung des implementierten zweiwertigen Iones (meistens $\mathrm{Ca}$ oder $\mathrm{Sr}$ ) einbezogen werden. Offensichtlich wird bei der Substitution nicht nur die Valenzbilanz geändert, sondern es ändert sich dabei (lokal) auch die Struktur des Phononenbandes. Durch die Fehlanpassung des Ca-Ions an die benachbarte Umgebung bekommt das Gitter eine lokal geänderte Struktur. Es kann daher zu einem strukturellen Selbsteinfang kommen, (,self trapping“). Selbst wenn lokaler DA eintritt, gibt es noch keine metallische Leitung. Dies bedeutet auch, dass die Konzentration der freien Zener's Ladungsträger dem Dotierungswert nicht gleich ist, zumindest bei den kleinen Dotierungen. Um einen freien Ladungsträger $\mathrm{zu}$ erzeugen, müssen die DA-Paare noch 
überlappen. Es ist auch unbekannt, wie viele benachbarte Alkali-Ionen für das „Einschalten“ der lokalen DA-Kopplung (durch eine weitere Änderung des Phononenspektrums) ausreichen.

Die Substitution eines Lanthanoid-Iones durch Strontium oder Calcium hat auch eine lokale Spinverzerrung zur Folge [24]. Ein anfangs antiferromagnetisch geordnetes Spinsystem wird nahe dem Fremdatom umgeordnet (siehe Abbildung 4.8b)

a)

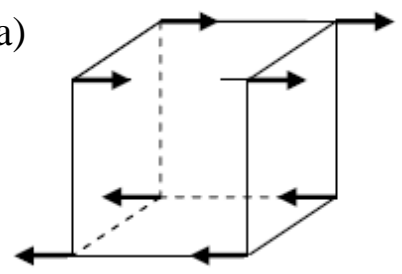

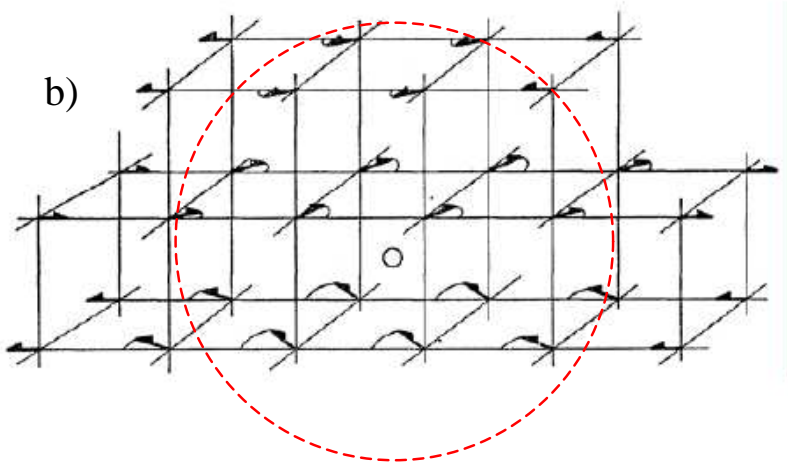

Abbildung 4.8: a) die ungestörte Spinanordnung in einem $\mathrm{RMnO}_{3}$-Manganit $\left.(\mathrm{R}=\mathrm{Nd}, \mathrm{La}, \ldots) ; b\right)$ lokale Spinänderung durch Implementierung eines Lochs (z.B. erzeugt durch Dotierung mit Strontium oder Calcium). Nach [24].

Bei den kleineren Konzentrationen sind diese umgeordneten Bereiche in eine Matrix von ungestörten $\mathrm{Mn}^{+3}$-Ionen eingebaut und voneinander isoliert. Die Orientierung der ferromagnetischen Komponenten dieser Bereichen ist wahrscheinlich über alle möglichen Richtungen statisch verteilt, sodass das Gesamtmoment Null ist (Spinglasähnlich). Erst bei höheren Dotierungswerten kommen diese Bereiche zur Überlappung. Sind die einzelnen Cluster nicht mehr unabhängig, so geht das System über den kohärenten gekanteten Zustand in den ferromagnetischen metallischen Zustand über, wobei die Doppelaustauschwechselwirkung eine dominierende Rolle spielt. Bei der $\mathrm{La}_{1-\mathrm{x}} \mathrm{Ca}_{\mathrm{x}} \mathrm{MnO}_{3}$-Verbindung wird z.B. schon bei $\mathrm{x} \approx 0.15$ und höher bis $x \approx 0.5$ eine ferromagnetische Ordnung beobachtet [57]. Ein gekanteter afm-Bereich wird bei der geringen Dotierung fast immer beobachtet, sofern keine Phasenseparation afm-fm eintritt. Die Kantung bei Antiferromagneten kann aber auch andere Ursachen haben, z.B. anisotroper Austausch Dzyaloshinskiy-Moriya.

\section{Phasenseparation}

Experimente an Manganiten haben gezeigt, dass in den meisten Fällen eine Phasenseparation stattfindet (Zerfall in ferro- und antiferromagnetische Bereiche). Es folgt daraus aber nicht, dass der gekantete Zustand niemals auftritt. Bevor wir ein Kriterium formulieren können - wann und welcher Zustand eintritt, wird die Phasenseparation betrachtet. Zuerst kann man sagen, dass es immer eine chemische Probeninhomogenität gibt (z.B. wegen der ungleichmäßigen Verteilung der dotierten Alkali-Ionen). Diese Art von Clusterbildung wird praktisch nicht von äußeren Faktoren beeinflusst (z.B. Temperatur, magnetisches oder elektrisches Feld, Druck usw.), und ist damit eine individuell fixierte Eigenschaft jeder Probe. Es gibt aber auch eine intrinsische magneto-elektronische Phasenseparation, z.B. in ferro- und antiferromagnetische Bereiche. Die 
Phasenanteile können in diesem Fall durch äußere Kräfte geändert werden. In der Literatur werden zwei Mechanismen diskutiert.

1. Elektronische Phasenseparation bei festen (eingefrorenen) Positionen der dotierten Ionen [64].

2. Phasenseparation, die durch die Diffusion der dotierten Ionen zustande kommt $[65,66]$.

Diese beiden Mechanismen werden aktiviert, wenn für die Ladungsträger der ferromagnetisch geordnete Zustand energetisch günstiger ist als der antiferromagnetische. Deswegen versuchen sie eine ferromagnetische Ordnung einzustellen. Dies kann nur dann gelingen, wenn die Ladungsträgerkonzentration genügend groß ist. Bei den kleineren Konzentrationen können sich die Ladungsträger dann nur in Einzelbereichen sammeln. Dort wird dann ferromagnetische Ordnung eingestellt, während der Restteil des Kristalls antiferromagnetisch bleibt. Es entsteht ein mesoskopisches System.

Im Falle der Phasenseparation des ersten Typs (elektronische Phasenseparation) führt die Konzentration der Ladungsträger in den Einzelbereichen zur Entstehung von lokalen Raumladungen (nur im isolierenden Zustand), während die Akzeptor- oder Donatorbeimischung gleichmäßig verteilt bleibt. So entstehen starke Coulomb-Felder in der Probe, die die ferromagnetischen und antiferromagnetischen Einzelbereiche wieder zu vermischen versuchen, um damit Coulomb-Energie zu gewinnen. Bei einer kleinen Konzentration der Ladungsträger bleiben die hochleitenden ferromagnetische Bereiche voneinander isoliert innerhalb der nichtleitfähigen antiferromagnetischen Matrix. Der Kristall zeigt dabei insgesamt ein isolierendes Verhalten. Bei der Erhöhung der Ladungsträgerkonzentration vergrößert sich der ferromagnetische Phasenanteil und ab einer bestimmten Konzentration perkolieren die ferromagnetischen Bereiche („Perkolationsschwelle“). Bei weiterer Konzentrationserhöhung geht der gesamte Kristall in die ferromagnetische metallische Phase über. Gehen wir noch weiter mit der Konzentrationserhöhung: wir wissen, dass z.B. $\mathrm{SrMnO}_{3}$ (maximale Konzentration der Löcher) antiferromagnetisch und isolierend ist. Dies bedeutet aber, dass sich der Kristall auf diesem Weg vom ferromagnetischen in den antiferromagnetischen Zustand umwandelt. Im Rahmen der Phasenseparationstheorie würde man diese Umwandlung folgendermaßen beschreiben: Irgendwann entstehen im ferromagnetischen Kristall einzelne antiferromagnetische Bereiche, die mit steigendem $\mathrm{x}$ anwachsen. Es gibt selbstverständlich auch eine Perkolationsschwelle für diesen Bereich, diese ist aber in den meisten physikalischen Eigenschaften nicht so stark ausgeprägt, wie die Perkolation der metallischen Bereiche. Letztlich (bei $\mathrm{DMnO}_{3}$, D ist ein Alkaliikon) wird das ganze Kristallvolumen antiferromagnetisch und isolierend. Ähnliche Phasenseparation findet man nicht nur bei den Manganiten. Z.B. ist die Phasenseparation auch bei den stark dotierten antiferromagnetischen Halbleitern EuSe und EuTe zu finden [64].

Eine anderer Typ von Phasenseparation ist der mit gleichzeitiger Diffusion der Beimischung. Dieser tritt nur bei hohen Temperaturen auf, und wird von zwei Faktoren bestimmt [64-66]:

1. Durch die gegenseitige Wechselwirkung der beigemischten Ionen entsteht eine Tendenz, die Abstände zwischen den Beimischungen so einzustellen, dass die Gesamtenergie minimal wird. Wenn die mittlere Konzentration der Beimischung kleiner ist als die optimale Konzentration, 
dann ist es für diese Art der Beimischung energetisch günstiger, sich in Einzelbereichen zu konzentrieren.

2. Die Tendenz der Ladungsträger, eine ferromagnetische Ordnung zu schaffen (ähnlich wie bei der elektronischen Phasenseparation), zwingt die Löcher (oder Elektronen) sich zu konzentrieren. Dabei sammeln sich auch die diese Ladungsträger erzeugenden beigemischten Ionen an.

Der größte Unterschied zwischen den beiden Phasenseparationsszenarien (elektronische und ionische Phasenseparation) ist die Abwesenheit von Raumladungen im zweiten Fall. Im Ergebnis kann die ferromagnetische Phase auf ziemlich große Bereiche wachsen, sogar bei kleinen Konzentrationen der Ladungsträger.

Welches Szenario der Phasenseparation sich in den Manganitsystemen tatsächlich abspielt, ist bis heute unklar. Eine Übersicht darüber findet man in [72]. Sicher ist nur, dass es sowohl die elektronische als auch die ionische Phasenseparation geben kann. Bei der ionischen Phasenseparation ist es problematisch anzunehmen, dass die Diffusion der dotierten Alkali-Ionen (Sr oder $\mathrm{Ca}$ ) wegen ihres relativ großen Ionenradius eine wichtige Rolle spielt. Es gibt aber auch die Meinung, dass es zu einer Separation durch Diffusion der Sauerstoffionen kommen kann [71]. Der Ionenradius des Sauerstoffes ist $\mathrm{R}_{\mathrm{O}}=66 \mathrm{pm}$, während z.B. der des Calciums $\mathrm{R}_{\mathrm{Ca}}=$ 197,3 pm beträgt. Es ist zur Zeit auch unklar, ob sich eine Separation ausschließlich zwischen der afm-isolierenden und fm-metallischen Phasen abspielt, oder ob die metallischen bzw. isolierenden gekanteten Phasen auch von Bedeutung sind.

\section{Pm- und fm-Cluster}

Wie man aus dem Phasendiagramm für die $\mathrm{Nd}_{1-\mathrm{x}} \mathrm{Sr}_{\mathrm{x}} \mathrm{MnO}_{3}-\mathrm{Manganite}$ (siehe Abb. 4.7) zu entnehmen kann, ist die $\mathrm{Nd}_{2 / 3} \mathrm{Sr}_{1 / 3} \mathrm{MnO}_{3}$-Verbindung unterhalb $\mathrm{T}_{\mathrm{C}}$ ferromagnetisch. Die magnetischen Messungen haben ebenso gezeigt (siehe Kapitel „Messergebnisse“), dass es dabei keine zusätzliche antiferromagnetische Komponente gibt. Eine antiferromagnetische Komponente bekommt man z.B. durch die Erhöhung des Sauerstoffdefizits $\delta$. NMRExperimente und X-Ray Synchrotron-Messungen an den stoichiometrischen $\mathrm{Nd}_{1-\mathrm{x}} \mathrm{Sr}_{\mathrm{x}} \mathrm{MnO}_{3^{-}}$ System haben die Existenz von Clustern mit geringer Ladungsträgerbeweglichkeit im metallischen Zustand unterhalb $\mathrm{T}_{\mathrm{C}}$ gezeigt $[85,86]$. Andererseits wird durch Untersuchungen der Transporteigenschaften an diesen Systemen [3] und durch Rastertunnelmikroskopie an dem $\mathrm{La}_{1-}$ ${ }_{\mathrm{x}} \mathrm{Ca}_{\mathrm{x}} \mathrm{MnO}_{3}(\mathrm{x} \approx 0.3)$ [4] die Existenz metallischer Cluster oberhalb $\mathrm{T}_{\mathrm{C}}$ belegt. In der Abbildung 4.9 (oben) wird das Phasengemisch für eine solche Situation schematisch dargestellt. Eine mögliche Erklärung für diesen ungleichmäßigen Phasenübergang der Probe in der Nähe von $\mathrm{T}_{\mathrm{C}}$ wäre eine inhomogene Verteilung der dotierten Alkali-Ionen ( $\mathrm{Sr}$ ) innerhalb der Probe. Bei der hohen Inhomogenität können sogar die antiferromagnetischen isolierenden Bereiche innerhalb der Probe auftreten, die der undotierten Verbindung entsprechen (z.B. $\mathrm{LaMnO}_{3}$ oder $\mathrm{CaMnO}_{3}$, siehe gelbe Bereiche in Abb. 4.9 oben). Der Übergang eines Clusters aus dem isolierenden pmZustand in den metallischen bei Temperaturänderung ist eine Folge der Konkurrenz zwischen dem delokalisierenden DE-Mechanismus und der lokalisierenden Elektron-Phonon-Kopplung. Die Clusterbildung und die damit verbundenen Perkolationseffekte machen es möglich, z.B. 
durch die Anwendung des hydrostatischen Druckes die Stärke der DA-Kopplung zu untersuchen [89].

\section{Blockierung der Phasenseparation}

In [54] werden die Bedingungen für eine mögliche Blockierung der elektronischen Phasenseparation diskutiert. Wenn die konkurrierenden Wechselwirkungen (DA und SA) ungefähr gleich sind und das mittlere Superaustauschintegral $\langle\mathrm{J}\rangle$ klein, so wird die freie Energie des Systems praktisch nur durch einen mischungsähnlichen Entropieterm bestimmt. In dieser Situation könnte sich dann der inkohärente deGennes Zustand realisieren. Diese Bedingung kann durch Änderung der Stärke von DA und SA z.B. bei der Doppelelementsubstitution (z.B. mit $\mathrm{Cr}^{+3}$ [14]) oder bei der Erhöhung des Sauerstoffdefizits erreicht werden. Gleichzeitig wird das System randomisiert, so dass sich eher ein frustrierter gekanteter Zustand realisierten wird. Ein weiteres Beispiel für ein randomisiertes System ist in [102] zu finden.

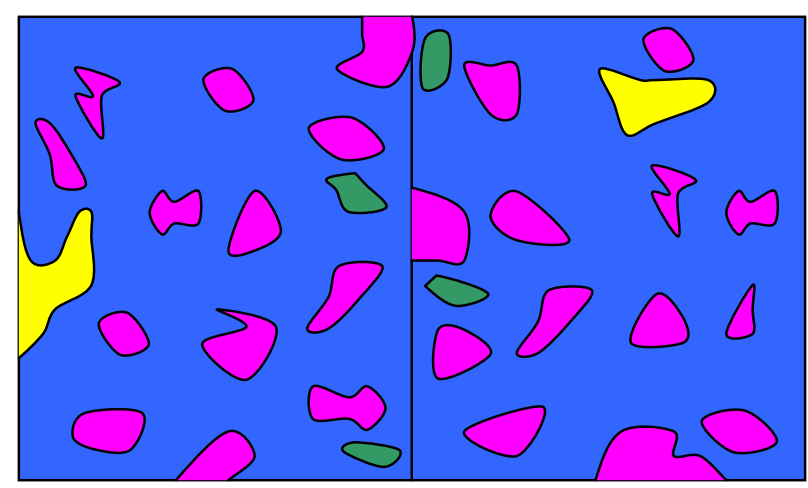

$\mathrm{T}<\mathrm{T}_{\mathrm{C}}, \delta=0$

$$
32
$$

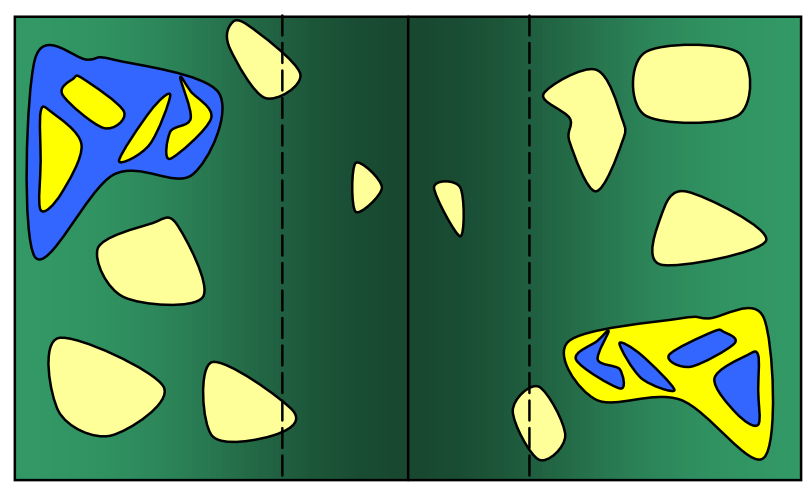

$\mathrm{T}<\mathrm{T}_{\mathrm{B}}, \delta>0$

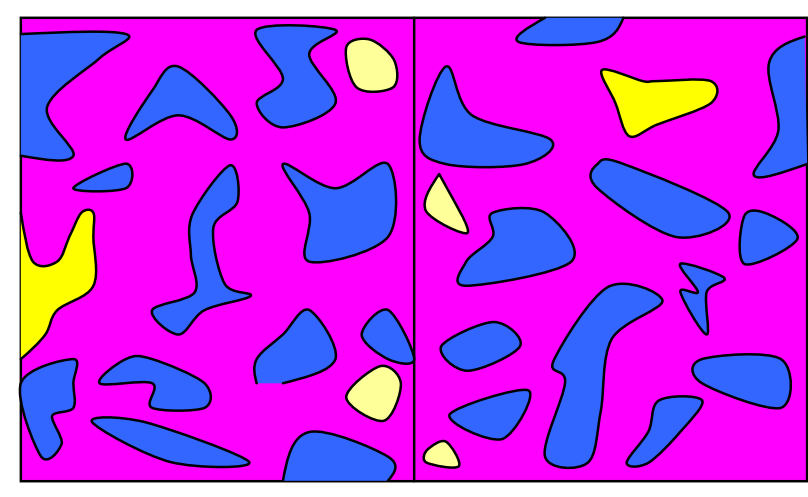

$\mathrm{T}>\mathrm{T}_{\mathrm{C}}, \delta=0$

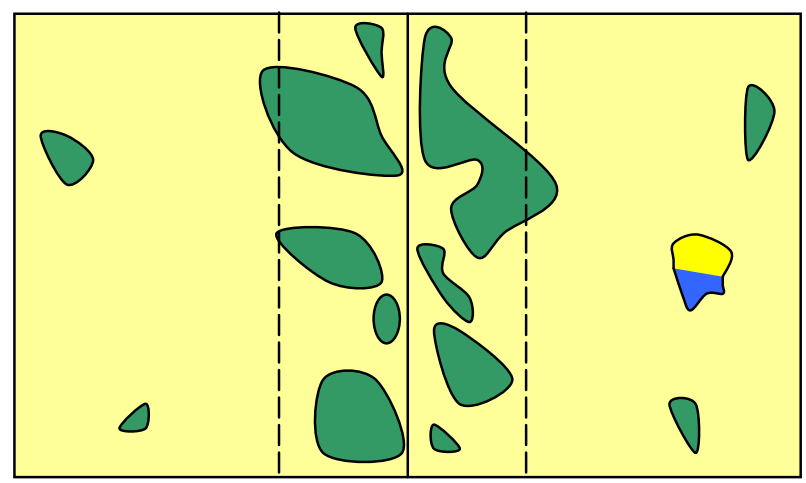

$\mathrm{T}>\mathrm{T}_{\mathrm{B}}, \delta>0$

Abbildung 4.9: Schematische Darstellung des sich verändernden mesoskopischen Systems.

$\square$ gekantete (sro) Phase; $\square$ antiferromagnetische (sro) isolierende Phase;

$\square$ antiferromagnetische isolierende Phase; $\square$ ferromagnetische metallische Phase;

$\square$ paramagnetische isolierende Phase.

Der Abbildung 4.9 (unten) zeigt anschaulich das Phasenseparationsszenario und die Ausbildung der gekanteten deGennes Phase für eine sauerstoffdefizitäre Manganitprobe. Bei der Probe mit $\delta$ 
$=0$ tritt in der Nähe von $\mathrm{T}_{\mathrm{C}}$ die Separation in die fm,m-Cluster und in die pm,i-Cluster ein. Bei der $\delta$-Erhöhung wird eine antiferromagnetische Komponente hinzugefügt. Bei den Temperaturen $\mathrm{T}_{\mathrm{B}}<\mathrm{T}<\mathrm{T}_{\mathrm{N}}$ (siehe das $\mathrm{T}-\delta$ Phasendiagramm, Abb. 4.18) befindet sich das System überwiegend im antiferromagnetischen Zustand. Bei den Temperaturen $T<T_{B}$ würde die Probe entsprechend der Phasenseparationstheorie in ferromagnetische und antiferromagnetische Bereiche zerfallen. Dies wird aber durch Randomisierung des Spinsystems verhindert, obwohl man es nicht ausschließen kann, dass es durch die inhomogene $\delta$-Verteilung zu lokalen phasenseparierten Bereichen kommen kann.

\subsection{Kopplung der Untersysteme in Manganiten}

Die Manganite zeigen ein breites Spektrum von physikalischen Eigenschaften, die für die Anwendung sehr interessant sind. Diese vielfältigen Eigenschaften weisen auf eine sehr hohe innere Komplexität dieser Systeme hin. Solche Materialien werden auch stark korrelierte Systeme genannt. Umfangreiche Experimente an den Manganiten zeigen, dass ihre magnetischen und elektrischen Eigenschaften eine Folge der Wechselwirkung von Spin-, Elektronen- und Phononenuntersystem sind [28-32]. Diese drei Untersysteme sind also stark gekoppelt. In der Abbildung 4.10 ist die gegenseitige Abhängigkeit aller drei Teilsysteme als ein „Kopplungsdreieck“ schematisch dargestellt.

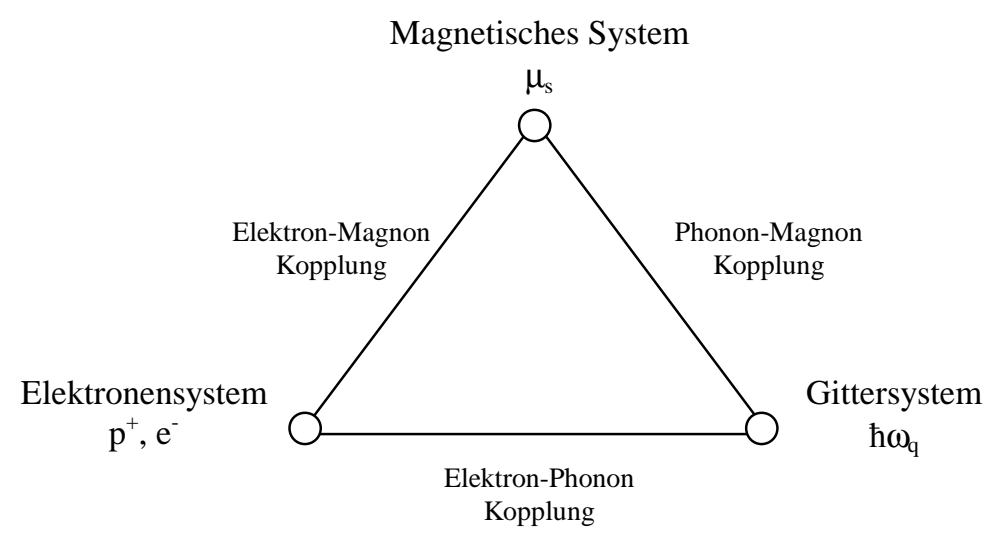

Abbildung 4.10: „Kopplungsdreieck“ - Schematische Darstellung der Wechselwirkungen zwischen den drei verschiedenen Untersystemen in einem Manganit

Jede Seite dieses Dreiecks entspricht einer bestimmten Kopplung und damit einem entsprechenden Kreis von physikalischen Phänomenen. Für das Zusammenwirken von Gittersystem und magnetischen System ist die sogenannte Phonon-Magnon Kopplung zuständig. Die Elektron-Magnon Kopplung ermöglicht eine Wechselwirkung zwischen dem magnetischen und dem elektronischen System und das Gitter ist durch die Elektron-Phonon-Kopplung mit dem elektronischen System verbunden. Wenn man also in einem dieser Untersysteme durch eine äußere Kraft irgendeine Änderung hervorruft, werden sofort alle anderen Untersysteme beeinflusst. Obwohl die äußeren Kräfte so Änderungen in allen drei Untersystemen induzieren, kann man fast immer einen sogenannten primären Angriffspunkt auszeichnen. 


\subsection{Die auf die Manganite wirkenden thermodynamischen Kräfte.}

\section{Entropische Kraft.}

Das schon längst bekannte Phänomen der Korrelation zwischen den magnetischen Eigenschaften und der Leitfähigkeit in den Manganiten ist ein gutes Beispiel dafür, dass zwei Untersysteme fast starr gekoppelt sind. In der Abbildung 4.11 wird diese Korrelation am Beispiel einer keramischen $\mathrm{Nd}_{2 / 3} \mathrm{Sr}_{1 / 3} \mathrm{MnO}_{3}$-Probe gezeigt. Für dieses Phänomen ist die Elektron-MagnonKopplung verantwortlich: sie vermittelt zwischen dem Spinsystem und dem Elektronensystem. Unter der Wirkung einer äußeren entropischen Kraft (also einer Temperaturänderung) geht das magnetische Untersystem bei einer Temperatur $\mathrm{T}_{\mathrm{C}}$ aus dem paramagnetischen in den ferromagnetischen Zustand über (ein magnetischer Phasenübergang). Man stellt dabei aber fest, dass die ferromagnetische Curie-Temperatur und die Temperatur eines Metall-IsolatorÜbergangs gleich sind $\left(\mathrm{T}_{\mathrm{C}}=\mathrm{T}_{\mathrm{mi}}\right)$. Das bedeutet, dass die Änderungen, die im magnetischen System gegeben sind, gleichzeitig die elektronischen Eigenschaften des Gesamtsystems beeinflussen und umgekehrt. Wir greifen also durch die Temperaturänderung primär am Spinsystem an und durch die vorhandene starke Magnon-Elektron Kopplung wird zusätzlich in die Eigenschaften des Elektronensystems eingegriffen.

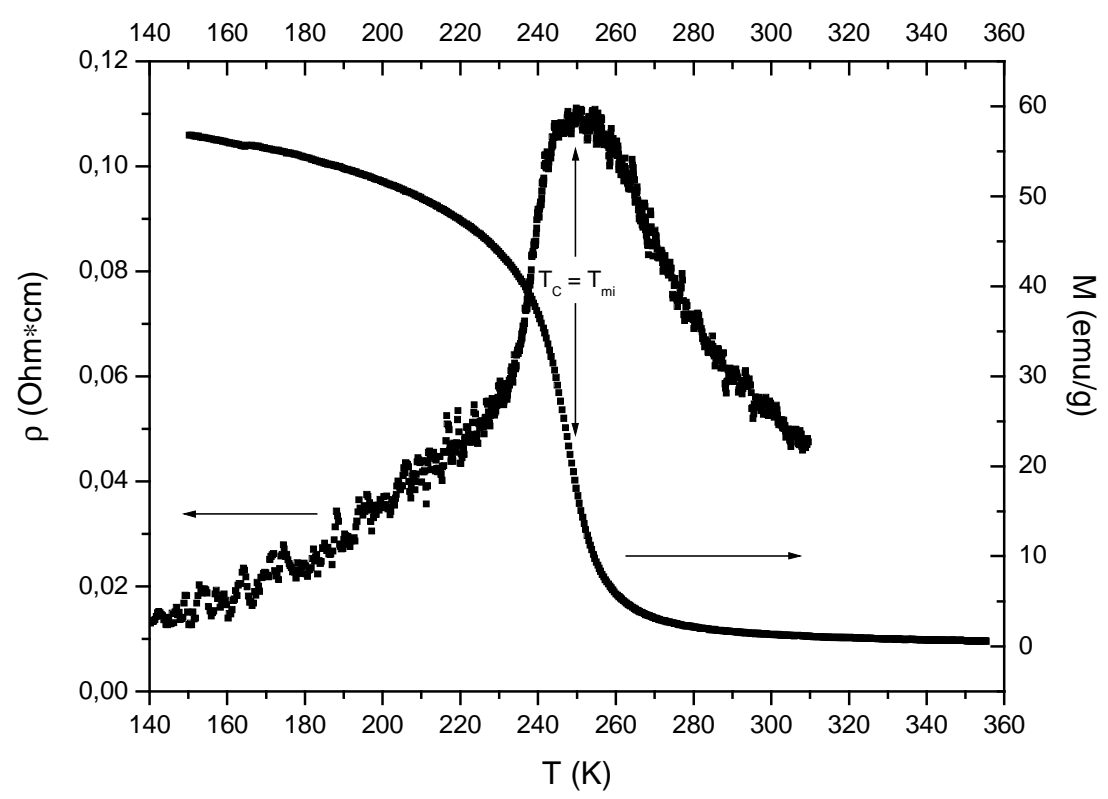

Abbildung 4.11: Korrelation zwischen den magnetischen und elektrischen Eigenschaften am Beispiel einer $\mathrm{Nd}_{2 / 3} \mathrm{Sr}_{1 / 3} \mathrm{MnO}_{3}$-Probe.

\section{Druckinduzierte Kraft.}

Setzt man eine Manganitprobe unter statischen Druck, so werden ihre magnetischen und elektrischen Eigenschaften durch den primär am Gitter angreifenden Druck geändert. Dies sieht man z.B. an den Messungen des Widerstandes unter verschiedener Druckbelastung (siehe Abb. 4.12). Mit diesem Beispiel wird primär die Kopplung zwischen Gittersystem und elektrischen Untersystem demonstriert. 


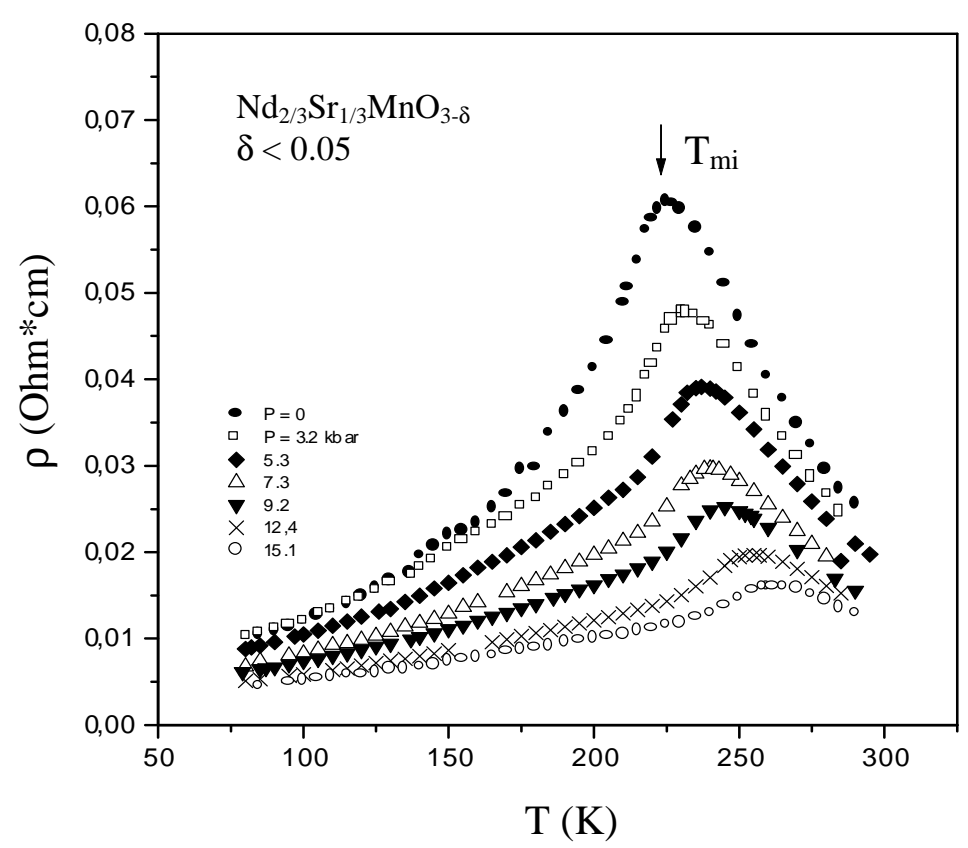

Abbildung 4.12: Abhängigkeit des Widerstandes einer keramischen $\mathrm{Nd}_{2 / 3} \mathrm{Sr}_{1 / 3} \mathrm{MnO}_{3-\delta}-\operatorname{Probe}(\delta<$ 0.05) vom angelegten hydrostatischen Druck. Nach [3].

Der Druck führt zur Erhöhung der Leitfähigkeit der Probe unterhalb und oberhalb des $\mathrm{T}_{\mathrm{mi}}{ }^{-}$ Punktes. Das Maximum von $\rho(T, P)$ wird kleiner und die Übergangstemperatur verschiebt sich in Richtung höherer Temperaturen. Welche Mechanismen spielen dabei eine Rolle? Die Leitfähigkeit hängt einerseits sehr stark ab von der Überlappung der Elektronenorbitale, die primär von den Strukturbesonderheiten bestimmt wird, sich unter Druck aber vergrößert. Andererseits spielt hier auch die Stellung der magnetischen Momente eine Rolle. Der spinabhängige Elektronentransfer wird i.A. durch Druckanwendung erleichtert. Existieren z.B. zwei Kopplungen (DA und SA), so wird bei einer Änderung der Überlappung der Elektronenorbitale auch die Bilanz zwischen der Doppelaustausch- und der Superaustauschwechselwirkung verändert, da beide abstandsabhängig sind. Damit werden wiederum die magnetischen Eigenschaften verändert. Die Überlagerung beider Effekte lässt sich auf zwei Wegen veranschaulichen. Man kann erstens die Größe der interpolierten Kationen ( R oder D) variieren (1). Damit induziert man einen sogenannten chemischen Druck. In diesem Fall werden aber nicht nur die Gitterparameter geändert, sondern es treten zusätzliche Effekte auf, die man der Änderung der Bindungswinkel zuordnet. Der andere Weg ist, die Probe unter statischen Druck zu setzen (2), wie in der Abbildung 4.12 gezeigt wird. In diesem Fall bekommt man primär eine Änderung der Bindungsabstände.

Als Ergebnis kann man die Leitfähigkeiterhöhung des Manganites in der metallischen Phase unter Druck der Zunahme der Übergangswahrscheinlichkeit teff zuordnen. Unter Druck wird die Überlappung der Elektronenorbitale stärker, die Bindungsabstände Mn-O-Mn werden kleiner und der Winkel der Mn-O-Mn-Bindung wird größer [36]. Dies führt zur Erhöhung des effektiven

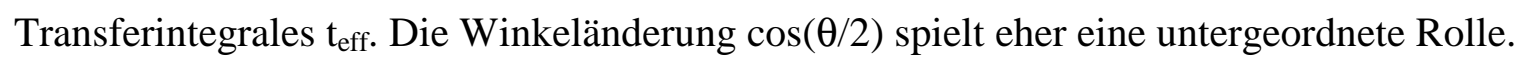


Die Hund'sche Wechselwirkung zwischen den lokalisierten $t_{2 \mathrm{~g}}$-Elektronen und den beweglichen $\mathrm{e}_{\mathrm{g}}$-Transportelektronen führt zu einer ferromagnetischen Spinausrichtung (DA). Das bedeutet, dass die Erhöhung der Wahrscheinlichkeit $t_{\text {eff }}$ unter Druck den ferromagnetischen Zustand der Probe stabilisiert, d.h. das ferromagnetisches Temperaturintervall wird unter Druck vergrößert $[44,45]$. Das wird als Verschiebung der $\mathrm{T}_{\text {mi }}$-Punktes zu höheren Temperaturen im Experiment sichtbar. Aus den experimentellen Daten dieser Arbeit wurde $\mathrm{dT}_{\mathrm{mi}} / \mathrm{dp}=2.14 \mathrm{~K} / \mathrm{kbar}$ bestimmt. Dieser Wert liegt nah am Wert $\mathrm{dT}_{\mathrm{mi}} / \mathrm{dp}=2.2 \mathrm{~K} / \mathrm{kbar}$ für die äquivalente $\mathrm{La}_{2 / 3} \mathrm{Ca}_{1 / 3} \mathrm{MnO}_{3-\delta^{-}}$ Verbindung [37], wie erwartet.

\section{Feldkraft.}

Eine weitere Möglichkeit, das Manganitsystem zu beeinflussen, gibt uns das magnetische Feld H. Der Probenwiderstand in einem magnetischen Feld nimmt bei den Manganiten sehr stark ab (kolossaler negativer Magnetowiderstand, CMR). In einigen Fällen reduziert sich der Widerstand um viele Größenordungen. Besonders groß ist dieser Effekt in der Nähe des Curie-Punktes, der durch das Anlegen eines H-Feldes ebenfalls zu höheren Temperaturen verschoben wird. In Abbildung 4.13 ist der magnetoresistive Effekt am Beispiel einer keramischen $\mathrm{Nd}_{2 / 3} \mathrm{Sr}_{1 / 3} \mathrm{MnO}_{3-\delta^{-}}$ Probe $(\delta=0.05)$ präsentiert.

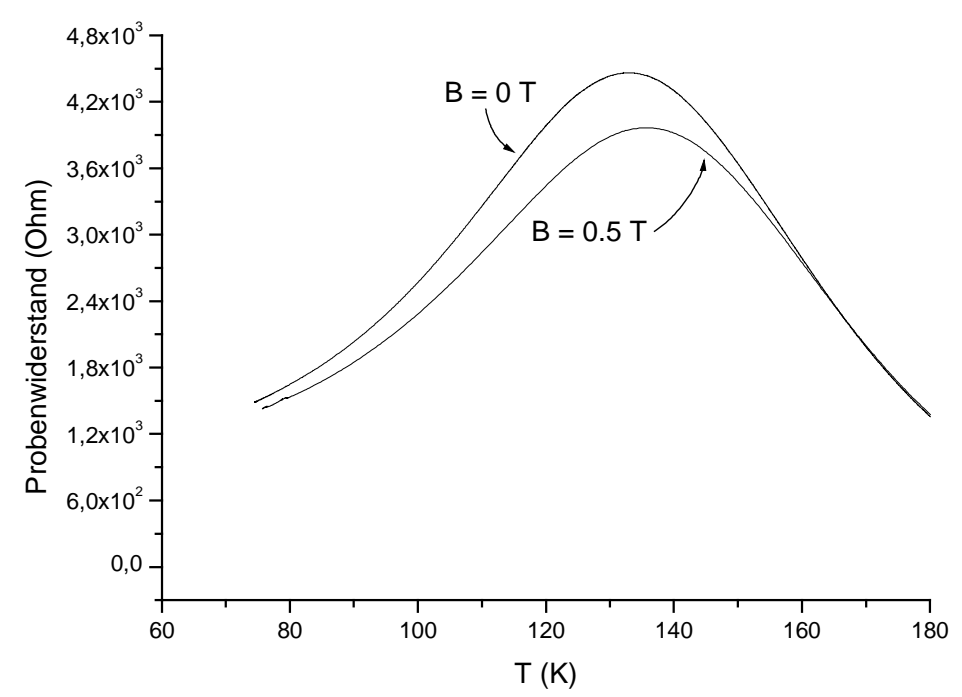

Abbildung 4.13: Einfluss des magnetischen Feldes auf die Leitfähigkeitseigenschaft (Magnetowiderstand) an dem Beispiel einer keramischen $\mathrm{Nd}_{2 / 3} \mathrm{Sr}_{1 / 3} \mathrm{MnO} \mathrm{O}_{3-\delta}$ Probe $(\delta=0.05)$.

Der negative magnetoresistive Effekt kann ebenfalls mit Hilfe des „Doppelaustausch“-Modells qualitativ erklärt werden. Bei einem angelegten H-Feld werden die Spinmomente der Mn-Ionen in Richtung des Feldes stärker ausgerichtet. Dies erhöht die Wahrscheinlichkeit teff des Doppelaustauschprozesses und die Leitfähigkeit der Probe nimmt daher zu. Die Verschiebung der Metall-Isolator-Übergangstemperatur wird wieder durch die Stabilisierung der ferromagnetischen Phase erklärt, hier aber unter dem angelegten magnetischen Feld. Im Fall des Magnetowiderstandes ist selbstverständlich das Spinsystem für das Magnetfeld der primäre Angriffspunkt, wie im Fall der entropischen Kraft. Die Änderungen in den elektronischen Eigenschaften werden durch die Wechselwirkung Spinsystem-Elektronensystem hervorgerufen. 
Die Analyse der Temperatur- und Feldabhängigkeit des Widerstandes [46, 47] führt zu der Aussage, dass die Leitfähigkeit i.W. nur von der Magnetisierung der Probe abhängig ist und nicht davon abhängt, wie man die Magnetisierungsänderung induziert hat - durch eine Temperaturänderung, das Anlegen des H-Feldes oder durch den hydrostatischen Druck. Es sind aber bei allen Überlegungen noch mesoscopische Effekte zu beachten, die auf elektronische Phasenseparation zurückzuführen sind. Phasenseparationseffekte werden von einigen Autoren sogar in den Vordergrund gestellt. Aber auch eine Phasenmischung kann nur die Eigenschaften der homogenen Phasen wiederspiegeln.

\section{Chemisches Potential.}

Primär werden die stoichiometrischen $\mathrm{R}_{1-\mathrm{x}} \mathrm{D}_{\mathrm{x}} \mathrm{MnO}_{3}$-Manganite untersucht. Schon geringe Abweichungen von der Stoichiometrie haben allerdings einen großer Einfluss auf ihre Eigenschaften [7, 12, 33, 34]. Dennoch sind heutzutage die dotierten bzw. sauerstoffdefizitären Systeme noch nicht genügend erforscht. Die Rolle, die z.B. der Sauerstoffdefizit in den Manganiten spielt, ist besonders erheblich. In der Arbeit [12] hat man zum erstenmal den Einfluss des Sauerstoffdefizites auf die Eigenschaften der La-Ca-Manganite systematisch untersucht. Dabei wurde festgestellt, dass die Magnetisierung mit zunehmenden $\delta$ generell kleiner wird, während sich die Curie-Temperatur zu tieferen Temperaturen verschiebt. Der Widerstand vergrößert sich dabei generell und die Maximumtemperatur der $\rho(T)$-Kurve wird kleiner.

Der Sauerstoffdefizit führt einerseits dazu, dass durch Änderung der Ladungsbilanz die Proportion $\mathrm{Mn}^{+3} / \mathrm{Mn}^{+4}$ geändert wird, und zwar so, dass die formale Lochkonzentration zunimmt. Dies sollte zur Erhöhung der Leitfähigkeit führen. Andererseits werden durch den Sauerstoffmangel die Zenerbindungen $\mathrm{Mn}^{+4}-\mathrm{O}^{-2}-\mathrm{Mn}^{+3}$ blockiert (,Zener bond blocking“). Diese beide Faktoren wirken gegeneinander und dies führt dazu, dass die Curie-Temperatur sich insgesamt in Richtung niedrigerer Temperaturen verschiebt, während der Widerstand zunimmt [7], d.h. die Erhöhung der Lochdotierung wird durch die Blockierung der Zenerbindungen [48] überkompensiert, und die Streuung der noch freien Ladungsträger an den Blockierungsstellen wird mit deren Anzahl großer.

Die Substitution der Mn-Ionen durch Eisen ist in seiner Wirkung einem Sauerstoffdefizit ähnlich - die Zenerbindungen werden dadurch zerstört [49]. In der Abbildung 4.14 sind dazu die Resistivitätskurven $\rho(\mathrm{T})$ einer Fe-dotierten und einer undotierten La-Ca-Manganitprobe (LCMO) gezeigt. Die nächste Abbildung 4.15 zeigt zum Vergleich den Widerstand von zwei O-dotierten Proben; dabei ist die eine fast stoichiometrisch $(\delta \approx 0.01)$ und die andere deutlich unstoichiometrisch $(\delta \approx 0.1)$ [6]. Auch bei diesen Kurven sind zwei Maxima und eine Verschiebung nach links $\mathrm{zu}$ finden. Das linke (sekundäre) Maximum wird einem Korngrenzeneffekt zu geordnet, während für das rechte (primäre) Maximum innere Eigenschaften des Manganits verantwortlich sind. In der Abbildung ist deutlich zu sehen, dass sich das linke Maximum mit der Sauerstoffdefizit stärker verschiebt als das rechte Maximum und auch seine Höhe nimmt schneller zu. Bei der Fe-Dotierung ist dies nicht der Fall. Das wird 
mit einem stärkeren Verlust des Sauerstoffes an den Korngrenzen in Verbindung gebracht. Bei dem Fe-dotierten System werden die Eisenionen offenbar eher homogen verteilt.

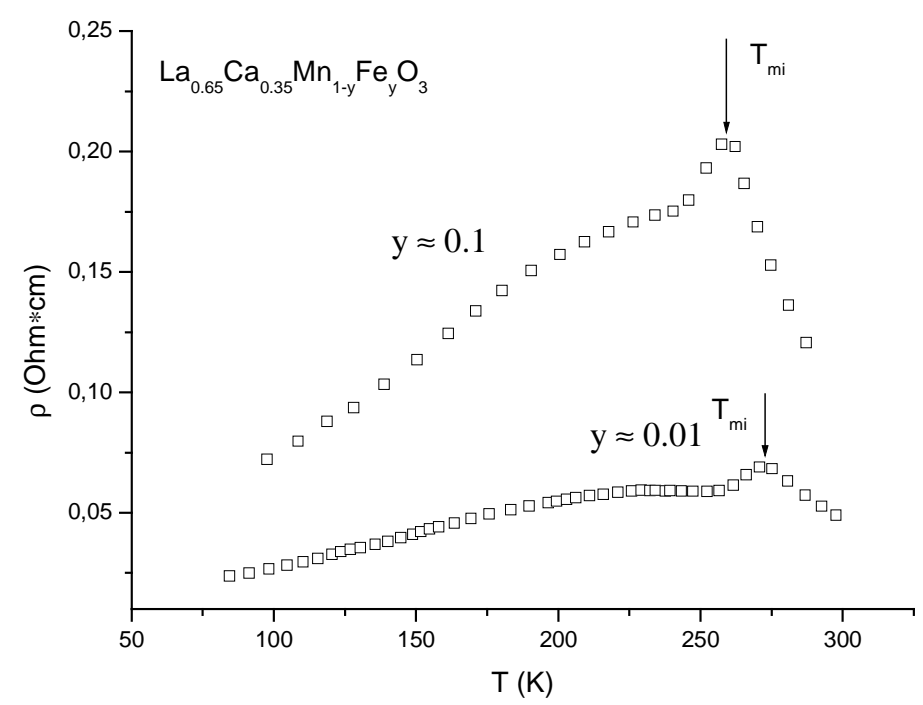

Abbildung 4.14: Der Widerstand der keramischen $\mathrm{La}_{0.65} \mathrm{Ca}_{0.35} \mathrm{Mn}_{1-y} \mathrm{Fe}_{y} \mathrm{O}_{3}$-Proben. Nach [49].

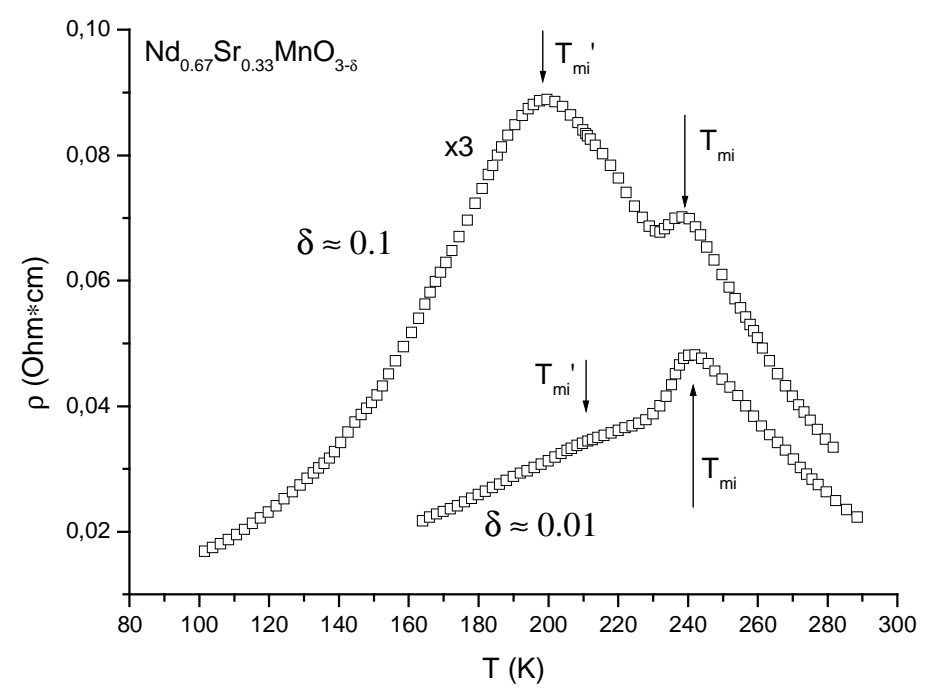

Abbildung 4.15: Widerstand der keramischen $\mathrm{Nd}_{0.67} \mathrm{Sr}_{0.33} \mathrm{MnO}_{3-\delta^{-}}$Proben. Nach [6]

Das Sauerstoffdefizit $\delta$ beeinflusst auch das Gitter. Mit zunehmendem $\delta$ wächst das Gittervolumen an. Dies ist vermutlich mit der Umwandlung $\mathrm{Mn}^{+3} \rightarrow \mathrm{Mn}^{+4}$ verbunden, da $\mathrm{Mn}^{+4}$ einen größeren Ionenradius hat.

Man kann bei Sauerstoffdefizit auch eine Verschiebung und Trennung der Stabilitätslinien der Phasenübergänge erwarten. Es gibt zum Beispiel Hinweise, dass in sauerstoffdefizitären Systemen die ferromagnetische Curie-Temperatur $\mathrm{T}_{\mathrm{C}}$ und die Temperatur des Metall-IsolatorÜberganges nicht mehr übereinstimmen, $\mathrm{T}_{\mathrm{C}}>\mathrm{T}_{\mathrm{mi}}[7,8,12,34]$, während sie bei den stoichiometrischen Verbindungen dank der starken Elektron-Magnon Kopplung gleich sind $\left(\mathrm{T}_{\mathrm{C}}\right.$ $=\mathrm{T}_{\mathrm{mi}}$ ). Diese Aufspaltung findet auch bei den Fe-dotierten Systemen statt [96]. 


\section{Elektrisches Feld und elektrische Dipole in Manganiten.}

Es gibt einige Manganitsysteme, deren Reichtum an Phänomenen selbst das übliche Maß überschreitet [4, 9, 51, 52]. Die Abbildung 4.16 zeigt dazu die Abhängigkeit des Widerstandes einer keramischen $\operatorname{Pr}_{0.7} \mathrm{Ca}_{0.3} \mathrm{MnO}_{3}$-Probe von der angelegten Spannung und Temperatur.

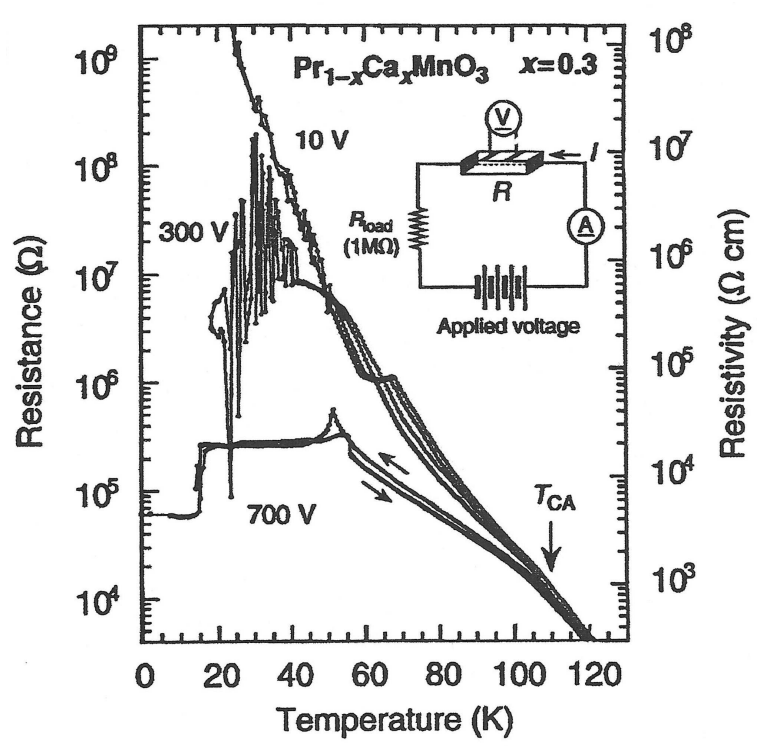

Abbildung 4.16: Abhängigkeit des Probenwiderstandes eines Manganites $\left(\mathrm{Pr}_{0.7} \mathrm{Ca}_{0.3} \mathrm{MnO}_{3}\right)$ von der Temperatur und dem angelegten elektrischen Feld. Nach [9].

Man sieht, dass hier sowohl das magnetische Feld als auch das elektrische Feld einen ungewöhnlich großen Einfluss auf die Probeneigenschaften haben. Damit das E-Feld das System überhaupt so stark beeinflussen kann, wird hier zusätzlich ein Untersystem elektrischer Dipole vermutet. Es ist bekannt, dass Perowskite nicht nur ferromagnetische sondern auch ferroelektrischen Eigenschaften besitzen. Es gibt viele perowskitähnliche Verbindungen, die nur ferroelektrische Eigenschaften zeigen (z.B. $\mathrm{BaTiO}_{3}$ ). Andererseits koexistieren oft magnetische und elektrische Dipole, sogenannte „Ferroics“. Selbstverständlich kann das Untersystem der elektrischen Dipole vom Restsystem nicht vollständig isoliert sein. Dann aber führen auch Änderungen in diesem Untersystem zu Änderungen in den anderen Untersystemen. In der Abbildung 4.17 wird daher ein neues „Kopplungsdreieck“ gezeigt; es enthält die gegenseitige Wechselwirkung zwischen dem magnetischen Untersystem, dem Elektronensystem, dem Gittersystem und dem System der elektrischen Dipole. Beide Dipolsysteme sind danach an das Elektronensystem und an das Gittersystem gekoppelt. Die direkte Kopplung zwischen dem magnetischen und dem elektrischen Dipolsystem ist entweder sehr schwach oder existiert gar nicht. In der Literatur werden zur Zeit keine Hinweise auf eine e-Dipol-Spin-Kopplung gefunden. Die Experimente zeigen aber (siehe „Messergebnisse"), dass der elektrische Widerstand vom elektrischen Feld E abhängt (elektroresistiver Effekt), ganz ähnlich wie vom Magnetfeld H. Dies bedeutet zumindest eine indirekte Wechselwirkung zwischen diesen beiden Systemen. 


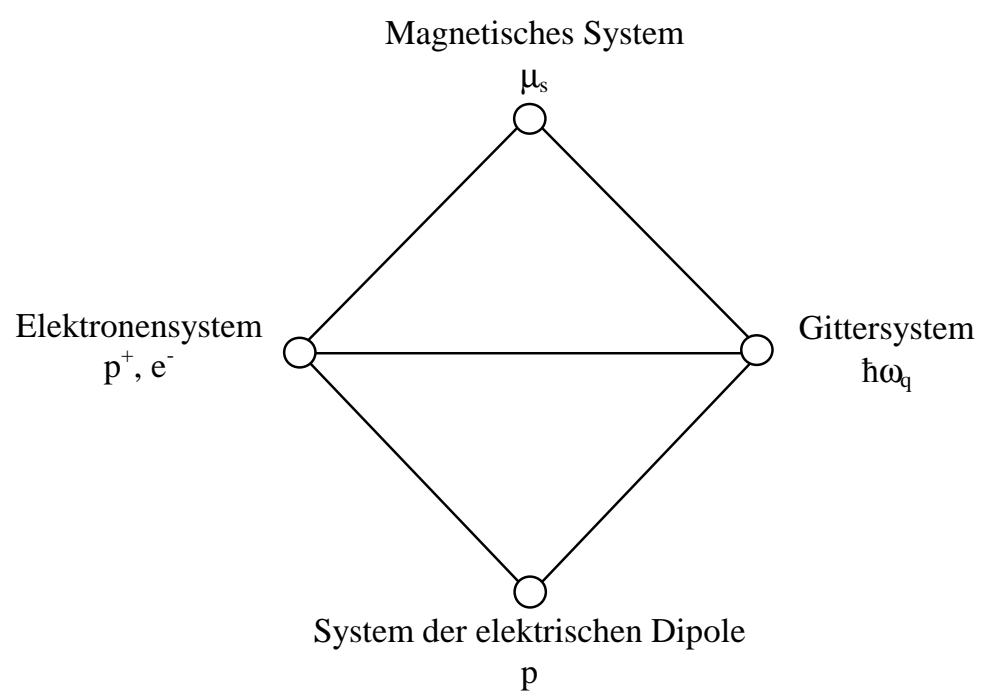

Abbildung 4.17: Schematische Darstellung der Wechselwirkung zwischen den vier verschiedenen Untersystemen (das magnetische Untersystem, das Elektronensystem, das Gittersystem und das System der elektrischen Dipole) in einem Manganit.

Damit elektrische Dipole überhaupt entstehen könnten, braucht man eine unsymmetrische Ladungsverteilung [53]. Wahrscheinlich spielt in bestimmten $\mathrm{R}_{1-\mathrm{x}} \mathrm{D}_{\mathrm{x}} \mathrm{MnO}_{3-\delta}$-Systemen das Sauerstoffdefizit die wesentliche Rolle bei der Entstehung von elektrischen Dipolen, vermutlich durch eine Rekonfiguration der O-Nahumgebung [54]. Dann würde man eine Korrelation zwischen dem Sauerstoffgehalt und der e-Dipoldichte bzw. den dipolbezogenen Effekten erwarten, wie auch beobachtet.

Es ist alternativ auch vorgeschlagen worden, dass die Ausbildung von Ladungsordnungsdomänen mit dem Auftreten elektrischer Dipole in Manganiten zu tun haben kann [54].

\subsection{Elektronische Zustände in sauerstoffdefizitären Manganiten}

Das deGennes Phasendiagramm (siehe Abb. 4.6) ist die Folge der Konkurrenz der Super- und der Doppelaustauschwechselwirkungen bei der Berücksichtigung der thermischen Entwicklung der Mittelwerte $\langle\cos (\theta)\rangle$ und $\langle\cos (\theta / 2)\rangle$. Dieses Diagramm zeigt, dass bei der kleinen Konzentration der Zener-Ladungsträger das System aus einem gekanteten Zustand in einen antiferromagnetischen Zustand bei einer Temperatur $\mathrm{T}_{1}$ übergeht und dann bei einer Temperatur $\mathrm{T}_{\mathrm{N}}$ paramagnetisch wird.

Abbildung 4.18 zeigt das T- $\delta$ Phasendiagramm, dass aus den im Rahmen dieser Arbeit durchgeführten Messungen gewonnen ist. Es ist zunächst überraschend, dass das T- $\delta$ Phasendiagramm dem deGennes Diagramm sehr ähnlich ist, wenn man die strukturchemische Koordinate $\xi=\mathrm{bx} /|\mathrm{J}| \mathrm{S}^{2}$ durch $-\delta$ ersetzt. Diese Ähnlichkeit wird einerseits durch die Reduktion der Ladungsträgerkonzentration durch die Verletzung der Goodenough-Bedingung bei Erhöhung des Sauerstoffdefizits $\delta$ [7] erklärt. Andererseits hat das Saustoffdefizit auch eine Wirkung auf 
das Transferintegral b, durch Änderung der Gitterparameter [25]. Die genaue Beziehung zwischen $\delta$ und bx ist unbekannt. Trotzdem kann man sagen, dass ein fehlendes Sauerstoffion mindestens einen freien Ladungsträger wegnimmt. Dies folgert man schon aus der abfallenden Tendenz von $\mathrm{T}_{\mathrm{C}}$ bei steigendem Defizit $\delta$. Gleichzeitig mit der Änderung der Ladungsträgerkonzentration erhöht das Sauerstoffdefizit die Unordnung im Spinsystem [97], so dass möglicherweise die gekantete Phase stabilisiert wird. Die Ähnlichkeit beider Diagramme gibt uns Anlass, das deGennes-Modell bei der weiteren Diskussion zu verwenden, zumindest bei den kleinen $\mathrm{x}$ - oder den großen $\delta$-Werten. Dort ist das System vermutlich bereits randomisiert und die Phasenseparation zurückgedrängt. In der Arbeit [15] wurde eine Erweiterung für das deGennes Modell vorgeschlagen, die wir in den folgenden Abschnitten verwenden.

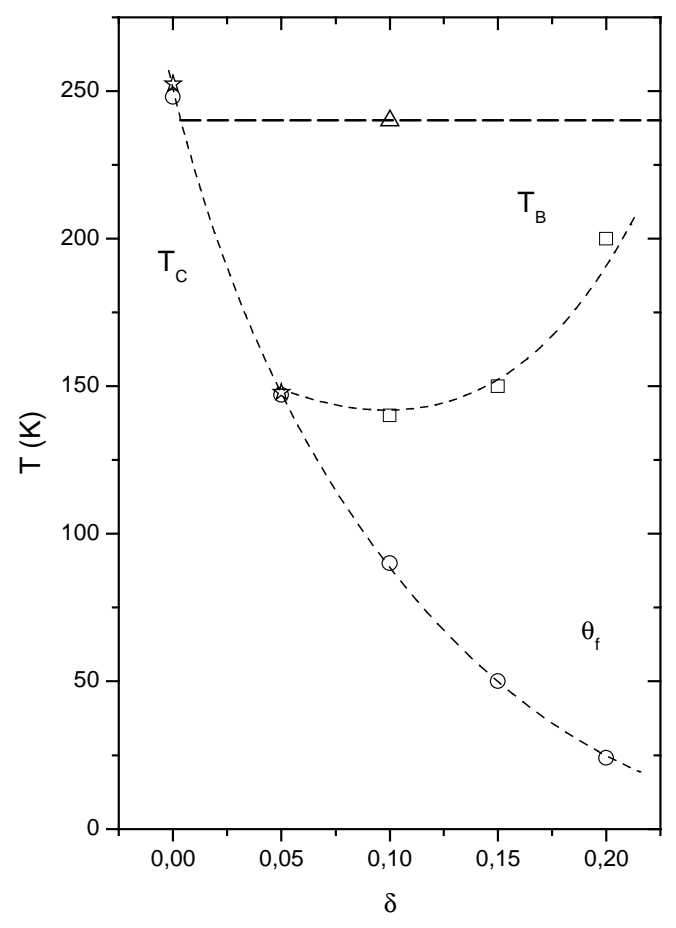

Abbildung 4.18: T- $\delta$ magnetisches Phasendiagramm von sauerstoffdefizitären $\mathrm{Nd}_{2 / 3} \mathrm{Sr}_{1 / 3} \mathrm{MnO}_{3-\delta^{-}}$ Manganiten.

Genaugenommen hat man zwei theoretische Modelle: elektronische Phasenseparation (1) und inkohärent gekantete deGennes-Phase (2), die jeweils nur einen kleinen Teil des Phasendiagramms (Abb. 4.18) abdecken. (1) ist nahe den Substanzen mit $\delta=0$ gültig, (2) nahe den Substanzen an der Stabilitätsgrenze für sauerstoffdefizitäre Manganite $\delta \leq 0.35$ [7]. Wir gehen jedoch davon aus, dass man aus der Analyse der beiden Grenzfälle auch für den Zwischenbereich Aussagen treffen kann.

\section{Elektronische Zustände in DA-SA Systemen}

Die freien Zener-Ladungsträger können in einem Leitungsband in der Brillouin Zone erfasst werden. Bei der Annahme, dass das Transferintegral spinabhängig ist, gilt für das einfache kubische Bandmodell in einer Richtung [34, 98]:

$E=E_{0}-J_{I} S \pm b \cdot \cos (\theta / 2) \cdot \cos (k a), 0<k<\pi / 2 a$ 
Im Unterschied zu der konventionellen Bandtheorie muss die Grenze der Brillouin Zone bei $k=$ $\pi / 2 a$ festgelegt werden (hier ist $a$ die Gitterkonstante der gewählten Richtung), weil sich in einem antiferromagnetischen Zustand die Periodizität des Gitters verdoppelt. Die elektronischen Zustände, die der Formel (4.8) entsprechen, sind in Abb. 4.19a schematisch dargestellt. In diesem Fall haben die „spin down“ und „spin up“ Zustände verschiedene Energien, die voneinander getrennt sind, im Gegensatz zum Pauli Band. Da wir hier ein spinabhängiges Band haben, ist es möglich, mit äußeren Kräften (T, H oder E) die Bandbreite zu variieren. Bei DA-SA Konkurrenz haben wir offenbar zwei Grenzfälle $\theta=0$ und $\theta=\pi$. Bei $\theta=\pi$ kollabieren die beiden Bänder (,spin up“ und „spin down“) in einen zweifach entarteten Zustand mit $E=E_{0}$ $J_{I} S$. Da die Beweglichkeit $\mu$ der Ladungsträger bei flachen Bändern sehr klein ist $(\mu \sim \mathrm{dE} / \mathrm{dk})$, wird das System bei $\theta=\pi$ zu einem antiferromagnetischen Isolator ( $\left.\mathrm{dE} /\left.\mathrm{dk}\right|_{\theta=\pi}=0\right)$, obwohl hier noch keine Bandlücke existiert. Bei der Änderung der magnetischen Struktur von einem gekanteten Zustand zu einem Antiferromagneten tritt hier also gleichzeitig ein Metall-IsolatorÜbergang auf, $\mathrm{T}_{1}=\mathrm{T}_{\mathrm{mi}}$.
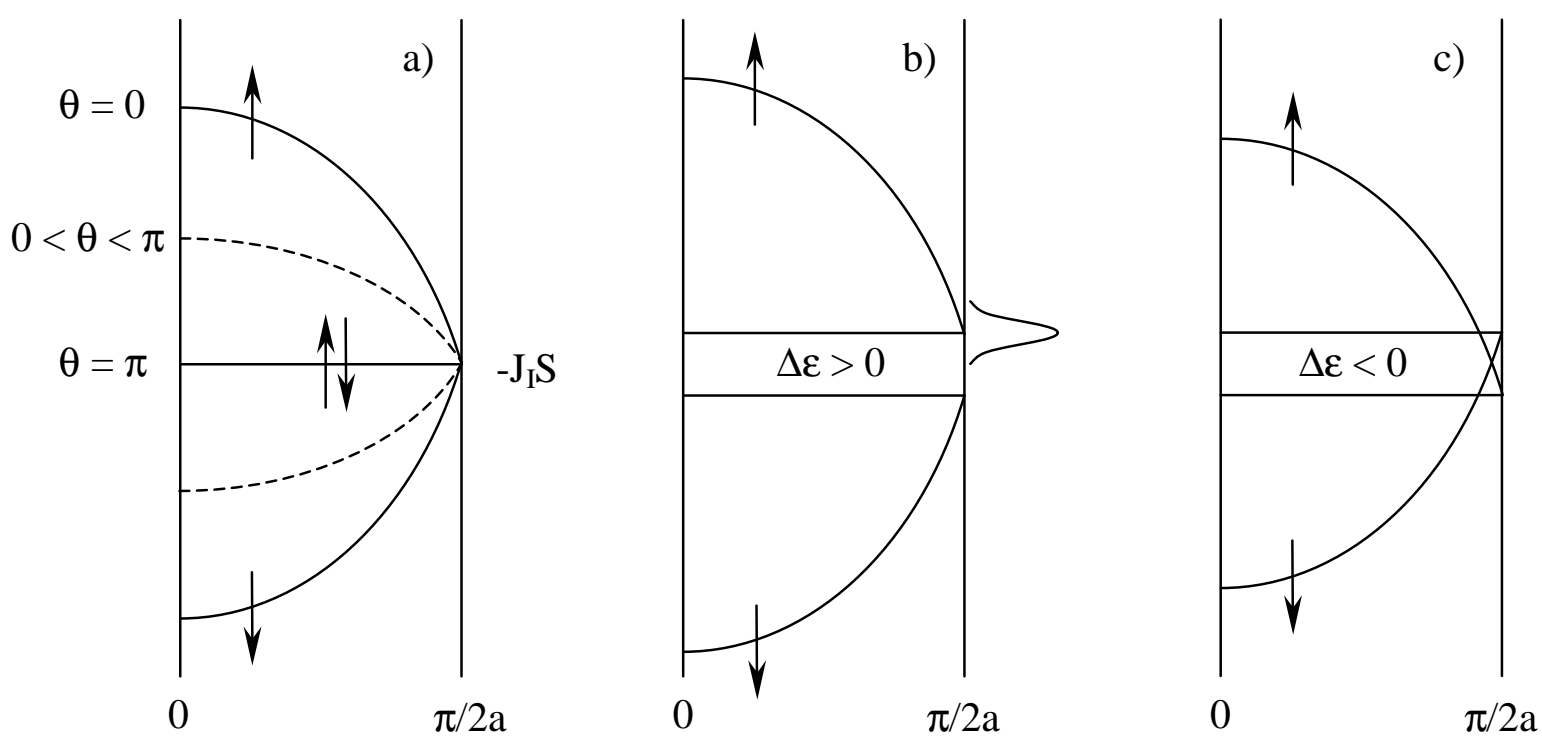

Abbildung 4.19: „Spin up“ und „spin down“ Energiebänder in einem Manganit. In b) und c) sind zwei mögliche Aufhebungen der Entartung gezeigt, die einer halbleitenden und einer halbmetallischen Situation entsprechen.

In einem Festkörper wird jedoch eine solche Enartung (bei $\theta=\pi$ ) durch Wechselwirkungen fast immer aufgehoben [99, 100]. Bei den Manganiten ist dies im Wesentlichen die ElektronPhonon-Wechselwirkung. Es kann allerdings auch die Spin-Bahn Kopplung oder die ElektronElektron Wechselwirkung eine Rolle spielen. Gleichzeitig mit der Aufspaltung werden dank statistischer Wechselwirkungen (magnetische und Gitteranregungen) beide kollabierten Bänder etwas verbreitert. Vor allem die Verbreiterung des oberen leeren Bandes spielt eine bedeutende Rolle beim Elektronentransport [15]. Dieser Fall wird in Abb. 4.19b dargestellt. Die Situation ist hier sehr ähnlich der Situation bei einem Halbleiter mit gut beweglichen Elektronen und etwas schwereren Löchern (Halbleitersitution). Für diesen Fall gilt: $\mathrm{T}_{1}=\mathrm{T}_{\mathrm{mi}}$. Wir haben aber keinen Grund, uns nur auf den Fall zu beschränken, in dem das „,spin up“ Band (bezüglich auf die hier 
angenommene Bezeichnung) bei der Entartungsaufhebung nach oben verschoben wird, während sich das „spin down“ Band entsprechend nach unten verschiebt. Es kann offenbar auch eine inverse Situation vorkommen (siehe Abb. 4.19c), wobei wir einen anderen Typ von Bandstruktur erhalten, ähnlich wie bei Halbmetallen. Von solch einer inversen Energielücke wurde bereits im Falle des $\mathrm{La}_{0.7} \mathrm{Ca}_{0.3} \mathrm{MnO}_{\mathrm{z}}$-Systems berichtet [101]. Während bei der Halbleitersituation die Energielücke praktisch sprungartig auftritt (wenn beide Bänder flach werden, bei $\theta=\pi$ ), wird sie im anderen Fall (Halbmetall) kontinuierlich geöffnet, bei kontinuierlicher Variation der Bandbreite durch äußere Kräfte.

\section{Erweitertes deGennes Diagramm für die Halbmetallsituation.}

Aus der Besonderheit der Bandstruktur im halbmetallischen Fall folgt die Spaltung der Ordnungstemperaturen $\mathrm{T}_{1}$ und $\mathrm{T}_{\mathrm{mi}}$. Bei einer Initialinvertierung der Bandkanten $\left(\varepsilon_{\mathrm{d}}>\varepsilon_{\mathrm{u}}\right)$ und der kontinuierlichen Änderung der spinabhängigen Bandbreite $b \cdot \cos (\theta / 2)$ durch eine äußere Kraft (z.B. Temperaturänderung) von $b$ zu $O$ öffnet sich die Energielücke, bevor die Flachbandkondition bei einer Temperatur $\mathrm{T}_{1}$ (c,i - afm,i Übergang) erreicht wird. Bei den Temperaturen oberhalb $\mathrm{T}_{\mathrm{mi}}$ und noch unterhalb $\mathrm{T}_{1}$ treten alle Elektronen (bzw. Löcher), die sich ursprünglich in dem ,spin-down“ Band befanden, nun in das „spin-up“ Band ein (siehe Abbildung 4.20a). Bei einem fast gefüllten Band (was bei den lochdotierten Manganiten der Fall ist) wird die Leitfähigkeit nicht durch die kaum beweglichen Löcher in einem fast flachen Band bestimmt, sondern durch die thermisch angeregten Elektronen in dem „spin-down“ Band. Die Abbildung 4.20b zeigt das modifizierte deGennes $\mathrm{T}-\mathrm{x}$ Phasendiagramm, wie es für den halbmetallischen Fall vorgeschlagen wurde [97].

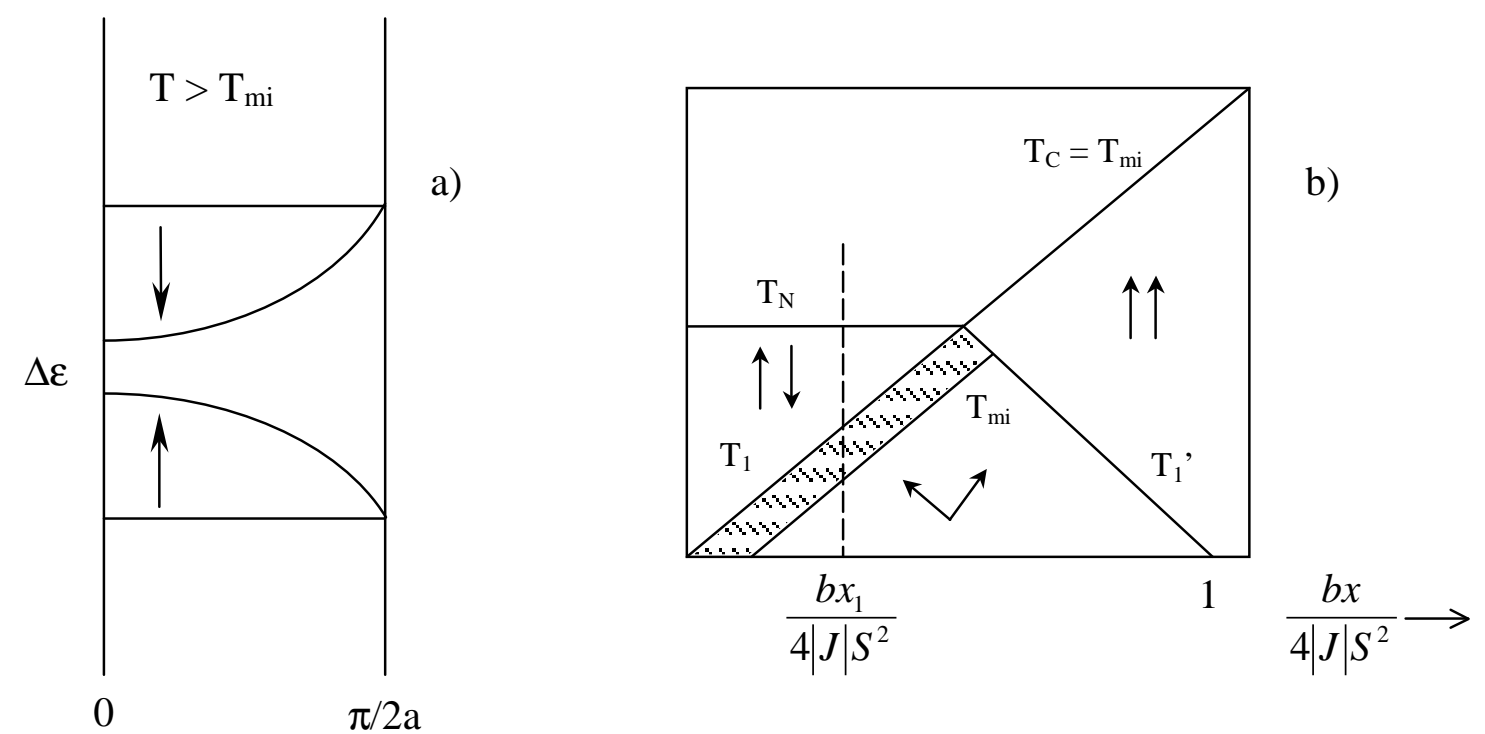

Abbildung 4.20: a) eine schematische Darstellung der Elektronenbänder in der Brilloin-Zone für den Bereich $T_{m i}<T<T_{1}$ (der gestrichelte Bereich in dem modifizierten deGenne's Diagramm); b) das erweiterte deGennes T-x Phasen Diagramm. Nach [15]. $T_{C}-$ die ferromagnetische Curie-Temperatur, $T_{N}$-die antiferromagnetische Neel-Temperatur, $T_{1}$ und $T_{1}$, - afm-gekantete und fm-gekantete Ordnung-Ordnung Phasenübergangstemperatur, $T_{m i}$-MetallIsolator Übergangstemperatur. 
Die Erweiterung der deGennes Ideen gibt uns nun die Möglichkeit, die magnetischen und elektronischen Zustände des hier zu untersuchenden Systems bei den hohen $\delta$-Weten zu beschreiben. Auf der linken Seite des T- $\delta$ Diagramms ( $\delta$ ist klein) kann sich der deGennesZustand vermutlich nicht realisieren: und das System bevorzugt dort ein Phasenseparationsszenario. Bei hohen $\delta$ wird der Phasenzerfall aber blockiert. Aus dem Vergleich des experimentellen T- $\delta$ Diagramm mit dem modifizierten deGennes Phasendiagramm können wir die $\mathrm{T}_{\mathrm{B}}$-Übergangstemperatur als $\mathrm{T}_{1}$ identifizieren, wobei ein $\mathrm{c}, \mathrm{i}-\mathrm{afm}, \mathrm{i}$ Phasenübergang stattfindet. Dem $\mathrm{T}_{\mathrm{mi}}$-Punkt wird entsprechend die paramagnetische CurieTemperatur $\theta_{\mathrm{f}}$ zugeordnet, wobei gleichzeitig ein Metall-Isolator Phasenübergang stattfindet $\left(\theta_{\mathrm{f}}\right.$ $=\mathrm{T}_{\mathrm{mi}}$.

\section{Verschiebung der $T_{1}$ und $T_{m i}$ Temperaturen im H-Feld.}

Wir wollen nun die Reaktion des hier zu untersuchenden Systems auf das Anlegen von äußeren H- und E-Feldern diskutieren. Das Anlegen des magnetischen Feldes hat die Änderung der mittleren Kantwinkel zu Folge und beeinflusst damit den Elektronentransfer. Dies wird z.B. in der Verschiebung der Übergangstemperaturen $\mathrm{T}_{1}$ und $\mathrm{T}_{\mathrm{mi}}$ im Feld sichtbar. Bei der Betrachtung des Systems im Magnetfeld muss man zur freien Energie den zusätzlichen Term -MH hinzufügen, die Zeeman-Energie. Für die Gesamtenergie gilt dann:

$E=E_{S A}+E_{D A}+E_{Z}$

Dabei sind $\mathrm{E}_{\mathrm{SA}}$ und $\mathrm{E}_{\mathrm{DA}}$ durch die Formeln (4.2) und (4.5) bestimmt (siehe Seite 54). Für $\mathrm{E}_{\mathrm{Z}}$ beim kohärent-gekanteten Zustand [24] bei Feldrichtung parallel zur Symmetrieachse der Magnetisierung der zwei Untergitter erhalten wir:

$E_{Z}=-H \cdot M_{0} \cdot \cos (\theta / 2)$

Aus der Minimierung der Gesamtenergie bei der Berücksichtigung des Entropieterms erhalten wir $[23,34]$ :

$<\cos (\theta / 2)>=H / H_{0}-T / T_{0}+x / x_{0}$

wobei $\mathrm{H}_{0}=4 \mathrm{Nz}|\mathrm{J}| \mathrm{S}^{2} / \mathrm{M}_{0}, \mathrm{~T}_{0}=4 \mathrm{Nz}|\mathrm{J}| \mathrm{S}^{2} / \alpha, \mathrm{x}_{0}=4|\mathrm{~J}| \mathrm{S}^{2} / \mathrm{b}$ und $\alpha$ ein Entropiekoeffizient ist. Bei der Temperatur $\mathrm{T}_{1}$ (gekantet-afm Übergang) wird $\langle\cos (\theta / 2)\rangle=0$. Einsetzen in Gl.3 ergibt für $\mathrm{T}_{1}(\mathrm{x}$, $\mathrm{H})$ :

$H / H_{0}-T_{1} / T_{0}+x / x_{0}=0$

Für eine bestimmte Probe $\mathrm{x}=$ const ist, bekommen wir für die Verschiebung von $\mathrm{T}_{1}$ :

$\mathrm{dT}_{1} / \mathrm{dH}=\mathrm{T}_{0} / \mathrm{H}_{0}>0$ 
Dies bedeutet eine Stabilisierung der gekanteten magnetischen Phase gegenüber der afm-Phase im magnetischen Feld. Dieses Ergebnis gilt für beide Szenarien (Halbleiter- und Halbmetallsituation), da bei der Ableitung keine Energielücke $\Delta \varepsilon$ benutzt wurde. Aus den experimentellen Daten ist das Anwachsen der $\mathrm{T}_{1}$-Temperatur mit dem zunehmenden $\mathrm{H}$-Feld deutlich zu erkennen. Trotzdem ist die genaue Identifizierung des $\mathrm{dT}_{\mathrm{B}} / \mathrm{dH}-$ Wertes nicht möglich, da die Verschiebung der Punkte sehr gering ist.

Wenn wir ein System haben, das zusätzlich zu den magnetischen noch die elektrischen Dipolmomente aufweist, die auch noch miteinander gekoppelt sind, so ist es möglich, den $\mathrm{T}_{1^{-}}$ Übergang auch mit dem elektrischen Feld zu beeinflussen. Man kann vermuten, dass die elektrischen Dipolmomente hier durch den O-Defizit entstehen [108, 111]. Zur Beschreibung der E-Feldwirkung haben wir den Term -PE zur Gesamtenergie hinzuzufügen. Wir bekommen nun eine Ähnlichkeit zwischen den E- und H-Feldern bezüglich der Wirkung auf das magnetische System. Trotzt dieser Äquivalenz sind aber verschiedene Suszeptibilitäten bei E und H-Feldern

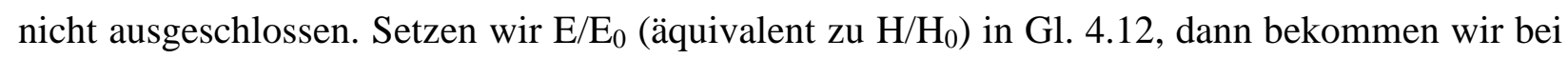
konstanten $\mathrm{H}$ und $\mathrm{x}$ :

$\mathrm{dT}_{1} / \mathrm{dE}=\mathrm{T}_{0} / \mathrm{E}_{0}>0$

Die steigende Tendenz stimmt mit den experimentellen Daten überein: $\mathrm{T}_{\mathrm{B}}$ steigt linear mit dem zunehmenden Messstrom, siehe Abb. 4.22.

Diskutieren wir nun den Metall-Isolator Phasenübergang bei der Temperatur $\mathrm{T}_{\mathrm{mi}}$. Dafür müssen wir die Energielücke $\Delta \varepsilon$, und ihre zwei möglichen Ausgangssituationen (halbleitend und halbmetallisch), in Betracht ziehen. Berücksichtigt wird auch der Einfluss des H- und/oder EFeldes (Stark- oder Zeeman-Effekt). Die Initialenergielücke $\Delta \varepsilon$ kann durch die magnetische Suszeptibilität der durch Anregungen verbreiterten antiferromagnetischen Flachbandzustände $\chi_{d}$ und $\chi_{u}$ erweitert werden, $\chi_{d} *$ und $\chi_{u} *$ sind die entsprechende Suszeptibilitäten im elektrischen Feld [15, 97]:

$\Delta \varepsilon(T, H, E)=\Delta \varepsilon_{u d}+\left(\chi_{u}-\chi_{d}\right) H^{2}+\left(\chi_{u}^{*}-\chi_{d}^{*}\right) E^{2}=\Delta \varepsilon_{u d} \pm\left|\Delta \chi_{u d}\right| \cdot H^{2} \pm\left|\Delta \chi_{u d} *\right| \cdot E^{2}$

Dies gilt für beide Situationen (halbmetallisch und halbleitend). Wir wollen jetzt die mögliche Reaktion der $\mathrm{T}_{\mathrm{mi}}$-Temperatur im halbmetallischen Fall auf die äußeren $\mathrm{H}$ - und E-Felder genauer betrachten. In diesem Fall öffnet sich die Energielücke bei einem Winkel $\theta_{\mathrm{mi}}<\pi$; dabei gilt:

$\cos \left(\theta_{m i} / 2\right)=\Delta \varepsilon / 2 b$

Setzen wir nun diese Gleichung in (4.11), dann erhalten wir die Abhängigkeit der $\mathrm{T}_{\mathrm{mi}}{ }^{-}$ Temperatur vom elektrischen und magnetischen Feld [97]:

$T_{m i} / T_{0}=x / x_{0}+H / H_{0}+E / E_{0}-\left(\Delta \varepsilon_{u d} \pm\left|\Delta \chi_{u d}\right| H^{2} \pm\left|\Delta \chi_{u d} *\right| E^{2}\right) / 2 b$ 
Nach der Gleichung (4.17) und (4.12) bewegen sich die $\mathrm{T}_{\mathrm{mi}}$ und $\mathrm{T}_{1}$ Temperaturen aufeinander zu, wenn $\Delta \chi_{\mathrm{ud}}$ (bzw. $\left.\Delta \chi_{\mathrm{ud}}{ }^{*}\right)<0$ ist, und voneinander weg bei $\Delta \chi_{\mathrm{ud}}$ (bzw. $\left.\Delta \chi_{\mathrm{ud}}{ }^{*}\right)>0$. Im Vergleich mit der $\mathrm{T}_{1}$-Temperatur ist die Verschiebung von $\mathrm{T}_{\mathrm{mi}}$ deutlich stärker $\left(\left|\mathrm{dT}_{1}\right| / \mathrm{dX}<\right.$ $\mid \mathrm{dT}_{\mathrm{mi}} / \mathrm{dX}, \mathrm{X}=\mathrm{E}$ oder $\left.\mathrm{H}\right)$. Abbildung 4.21 zeigt zwei mögliche Verhaltensweisen der $\mathrm{T}_{\text {mi- }}$ Temperatur als Funktion von $\mathrm{E}$ oder $\mathrm{H}, \Delta \mathrm{T}_{\mathrm{mi}}=\mathrm{f}(\mathrm{E}$ oder $\mathrm{H})$, entsprechend dem Vorzeichen von $\left|\Delta \chi_{\text {ud }}\right|$. Im zweiten Fall dT $\mathrm{mi}_{\mathrm{mi}} / \mathrm{dX}$ ändert sich ihr Vorzeichen von ,,+“ zu ,,-“ (wiedereinkehrendes Verhalten), während im ersten Fall $\Delta \mathrm{T}_{\mathrm{mi}}$ eine quadratische Funktion resultiert.

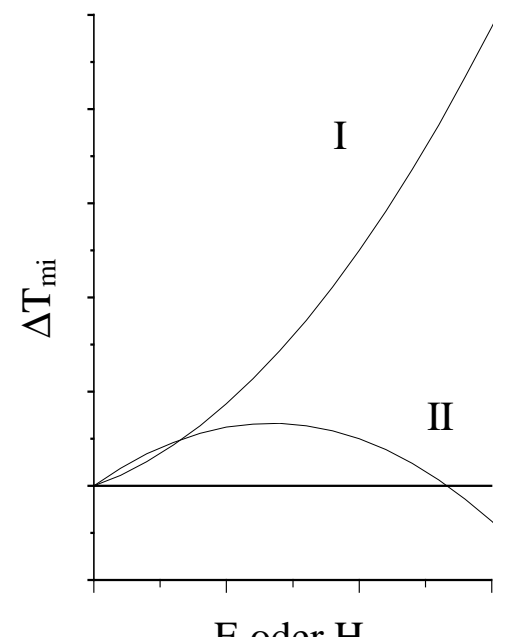

Abbildung 4.21: Zwei mögliche Fälle für die $\Delta T_{m i}$-Entwicklung im magnetischen oder

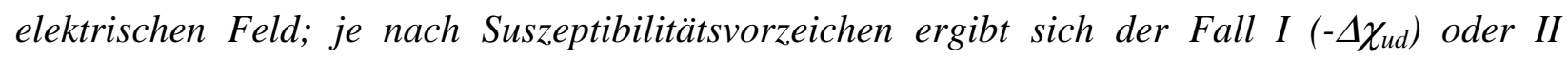
$\left(+\Delta \chi_{u d}\right)$.

Wenn wir jetzt die experimentell erhaltenen $\mathrm{T}_{\mathrm{mi}}$-Werte mit dem theoretischen Aussagen vergleichen, können wir für die $\mathrm{Nd}_{2 / 3} \mathrm{Sr}_{1 / 3} \mathrm{MnO}_{3-\delta}$-Probe (bei $\delta=0.05$, ,Messergebnisse“) das Szenario II feststellen (wiedereinkehrendes Verhalten). Für die Proben mit dem höheren Sauerstoffdefizit könnten die $\mathrm{T}_{\mathrm{mi}}$-Punkte den Messungen nicht entnommen werden. Wir können aber dort die Übereinstimmung des Experimentes mit der Theorie mit Hilfe der feldinduzierten Ordnung-Ordnung-Übergange überprüfen.

\section{Durch das E-Feld induzierte Ordnung-Ordnung Phasenübergänge.}

Die Messungen der elektrischen Widerstandes (Seite 32, „Messergebnisse“) ergeben Anomalien bei Temperaturen $T_{K}<T_{B}$, die nur ab einem bestimmten kritischen Messstrom $I_{c} z u$ finden sind. Zur Erklärung dieser Phänomene muss an dieser Stelle wiederholt werden, dass wir es hier mit einem Polykristall zu tun haben, wodurch die nah den Korngrenzen liegende Bereiche viel größere Sauerstoffdefizite $\delta$ haben als die Volumenbereiche. Durch das erhöhte Sauerstoffdefizit werden die Korngrenzenbereiche deutlich isolierender als das Volumen, sodass die gesamte angelegte Spannung praktisch nur an den Korngrenzen abfällt. Damit stehen sie unter dem Einfluss viel stärkerer E-Felder als die Volumenbereiche. Wir versuchen nun, die anomalen Änderungen des Widerstandes als eine Metallisierung der korngrenznahen Bereichen zu erklären, die durch das hohe elektrische Feld induziert wird. Im Rahmen der hier zu 
diskutierenden Theorie wird dann der $\mathrm{T}_{\mathrm{K}}$-Punkt als ein Metall-Isolator Übergang identifiziert. Die Reaktion dieser Anomalie auf elektrische und magnetische Felder muss dann zumindest ähnlich dem theoretischen $\mathrm{T}_{\mathrm{mi}}$-Verhalten sein.

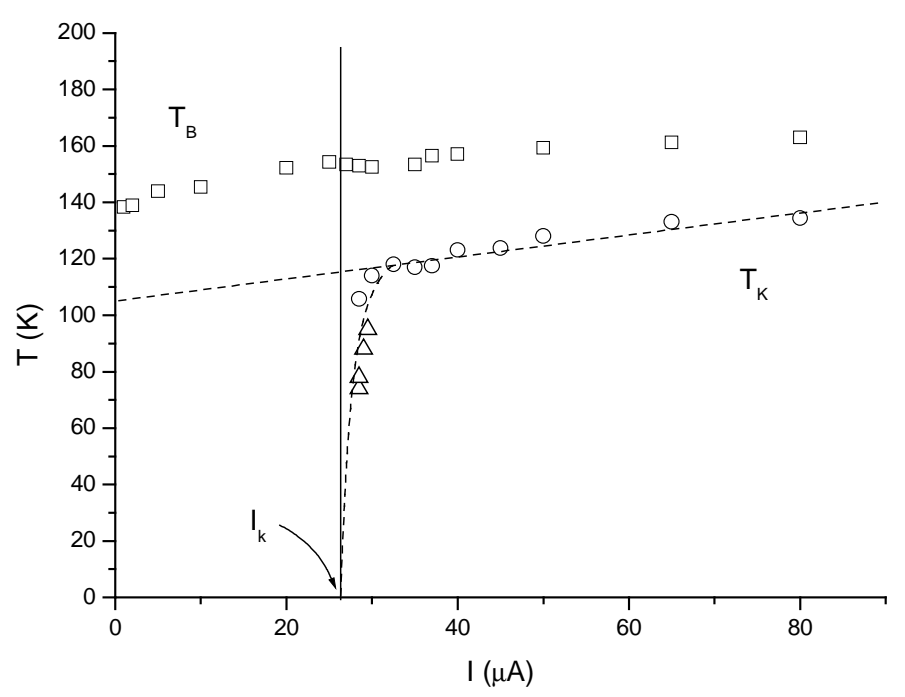

Abbildung 4.22: Das T-I Diafgramm einer $\mathrm{Nd}_{2 / 3} \mathrm{Sr}_{1 / 3} \mathrm{MnO}_{3-\delta}$ Probe $(\delta=0.15)$. Aus diesem Diagramm ist zu entnehmen, dass es für $T_{K}$ einen kritischen Schwellstrom $I_{K} \approx 26 \mu A$ gibt. Die runden und dreieckigen Symbole für $T_{K}$ sind verschiedenen Messungen $(\rho(T)$ und $V(I))$ entnommen.

Abbildung 4.22 zeigt dazu das T-I Phasendigramm für eine Probe mit $\delta=0.15$. Wie erwartet, ist die Temperatur $\mathrm{T}_{\mathrm{K}}$ vom eingeprägten Strom (und damit auch vom elektrischen Feld) abhängig. Wir sehen, dass bei hohem E-Feld $\mathrm{T}_{\mathrm{K}}$ linear mit dem zunehmenden Feld verläuft, was dem Fall I der Halbmetall-Szenario (Abb. 4.21) entspricht. Nach diesem Ansatz sollte aber $\mathrm{T}_{\mathrm{K}}$ bei beliebig kleinen Feldern existent bleiben, was aber nicht der Fall ist: die Anomalie verschwindet bei einer kritischer Stromschwelle von $\mathrm{I}_{\mathrm{K}} \approx 26 \mu \mathrm{A}$. Zur Lösung dieses zusätzlichen Problems verwenden wir die Idee über die mögliche E-Feld induzierte Kompression (bzw. Ausdehnung) des Zener's Bandes [97]. Dafür wird angenommen, dass das spinunabhängige Transferintegral b (und damit die Bandbreite) durch die äußeren Felder sehr stark beeinflusst werden kann, b(H, E). Dies kann z.B. eintreten, wenn die Zustände mit den großen Wellenvektoren $k$ eine andere Suszeptibilität $\chi$ als die Zustände mit kleineren $k$ haben. Durch die Bandkompression wird z.B. in der Literatur die anomale Stabilisierung der gekanteten Phase im $\mathrm{Nd}_{0.7} \mathrm{Sr}_{0.3} \mathrm{MnO}_{3}$-System erklärt [11]. Ähnliche Effekte sind auch in $\mathrm{La}_{0.7} \mathrm{Ca}_{0.3} \mathrm{MnO}_{3}$ zu finden [70].

Betrachten wir nun die Abhängigkeit $\mathrm{T}_{\mathrm{mi}}(\mathrm{E})$ unter der Berücksichtigung der Bandbreite, die vom elektrischen Feld abhängig ist, $b=b(E)$. Einerseits, wie schon oben diskutiert, verschiebt sich die $\mathrm{T}_{\mathrm{mi}}(\xi)$ )Linie bei Anlegen des elektrischen Feldes (siehe Abb. 4.23) auch ohne feldabhängige Bandbreite. Wenn aber die $\mathrm{T}_{\mathrm{mi}}$-Übergangstemperatur explizit von $\mathrm{b}$ abhängt (Gl. 4.16), müssen die $\Delta \mathrm{T}_{\mathrm{mi}}(\mathrm{E})$-Kurven entsprechend der Abhängigkeit $\mathrm{b}=\mathrm{b}(\mathrm{E})$ korrigiert werden. Die Abbildung 4.23 zeigt das deGennes Phasendiagramm in reduzierten Koordinaten $\xi=b x / 4|J| S^{2}$. Die strukturchemische Koordinate $\xi$ hängt aber nun vom E-Feld ab. Aus dem Experiment (die 
Messungen von $T_{K}$ bei hohen Stromwerten) sehen wir, dass $T_{m i}$ eine kontinuierliche Steigung zeigt. Dies bedeutet eine Verschiebung der $\mathrm{T}_{\mathrm{mi}}(\xi)$ )Linie im Feld nach oben (Szenario I in Abb. 4.21). Das Vorhandensein eines kritischen E-Feldes lässt sich dann durch $b=b(E)$ erklären; dabei ist b eine steigende Funktion von E, die ein Sättigungsverhalten hat. Die $\xi(T)-$ und $\mathrm{T}_{\mathrm{mi}}(\xi)$ Linien bewegen sich in einem steigenden Feld aufeinander $\mathrm{zu}$ und ab einem bestimmten kritischen Feld $E_{K}$ überkreuzen sie sich. Dann zeigt das System eine Temperatur $T_{K}$. Der $T_{K^{-}}$ Punkt nähert sich dank der Sättigung von $b=b(E)$ sehr schnell dem normalen Verhalten an, das im Wesentlichen durch die Verschiebung der $T_{m i}(x)$-Linie bestimmt wird. Es ist im Prinzip auch möglich, die Existenz des kritischen Feldes durch eine abfallende Funktion $b=b(E)$ zu erklären. In diesem Fall wird die sich nach links bewegende $\xi(\mathrm{T})$-Linie von der $\mathrm{T}_{\text {mi }}$-Linie eingeholt. In diesem Fall kann jedoch keine schnelle Annäherung an das lineare Verhalten bei hohen Strömen stattfinden.

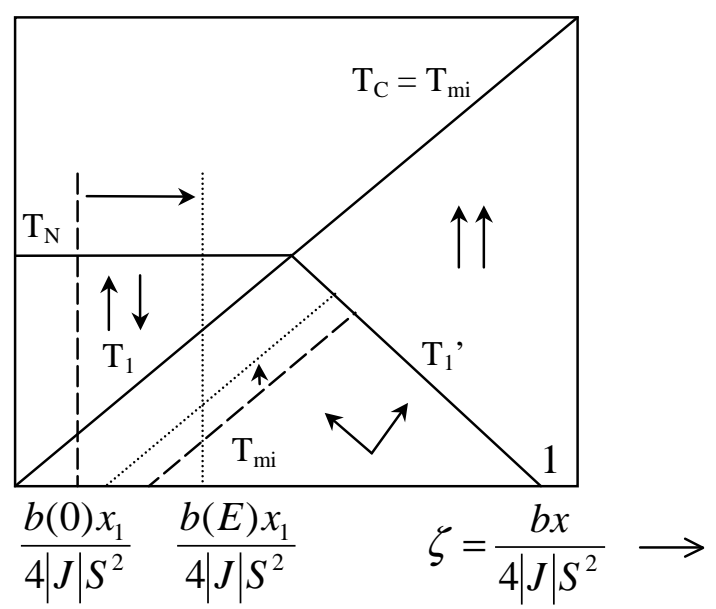

Abbildung 4.23: Das modifiziertes deGennes Phasendiagramm, wobei die $T_{m i}$ - und $\xi$-Linien vom angelegten E-Feld abhängig sind.

Wir wollen nun auch die Reaktion des $\mathrm{T}_{\mathrm{mi}}$-Punktes auf das magnetische Feld untersuchen. Der $\mathrm{T}_{\mathrm{K}}$-Übergang kommt nur bei einem bestimmten kritischen E-Feld zustande. Da der $\mathrm{T}_{\mathrm{mi}}$-Punkt dort bei tieferen Temperaturen liegt, werden nur Messungen benutzt, bei denen das magnetische und das elektrische Feld überlagert sind. Generell gibt es zwei Möglichkeiten: das Magnetfeld unterstützt das E-Feld oder wirkt ihm entgegen. Bei allen Proben mit hohen $\delta(0.15$ und 0.20, siehe Abb. 3.28 und 3.29) verschiebt sich der $\mathrm{T}_{\mathrm{K}}$-Punkt mit $\mathrm{H}>0$ in Richtung niedrigerer Temperaturen $\left(\mathrm{dT}_{\mathrm{K}} / \mathrm{dH}<0\right)$, d.h. entgegen dem E-Feld und das System wird so durch $\mathrm{H}$ in den gekanteten isolierenden Zustand zurückgeschoben (Antiäquivalenz).

\section{Intrinsischer elektroresistiver Effekt}

Es gibt offenbar drei Bereiche im Inneren der Probe, die man bei der Diskussion der elektroresistiven Effekte unterscheiden muss (siehe Abb 4.9). Das sind die Volumenbereiche (1), die an Korngrenzen angrenzende Bereiche (2) und die Korngrenzen selbst (3). Die oben schon diskutierte $\mathrm{T}_{\mathrm{K}}$-Temperatur spiegelt die Eigenschaften der Korngrenzenbereiche (2) wieder. Die Korngrenzen (3) prägen sich in den nichtlinearen Kennlinien V(I) (Kaltleiterverhalten, siehe 
Abb. 4.26a) aus. Wir versuchen aber, zunächst die elektroresistiven Effekte als Volumeneffekte zu verstehen. Wir wollen an dieser Stelle bemerken, dass für die Beschreibung der Bereiche (1) und (2) dieselbe Theorie benutzt werden kann, da sich die Bereiche 1 und 2 nur durch verschiedene $\delta$ unterscheiden.

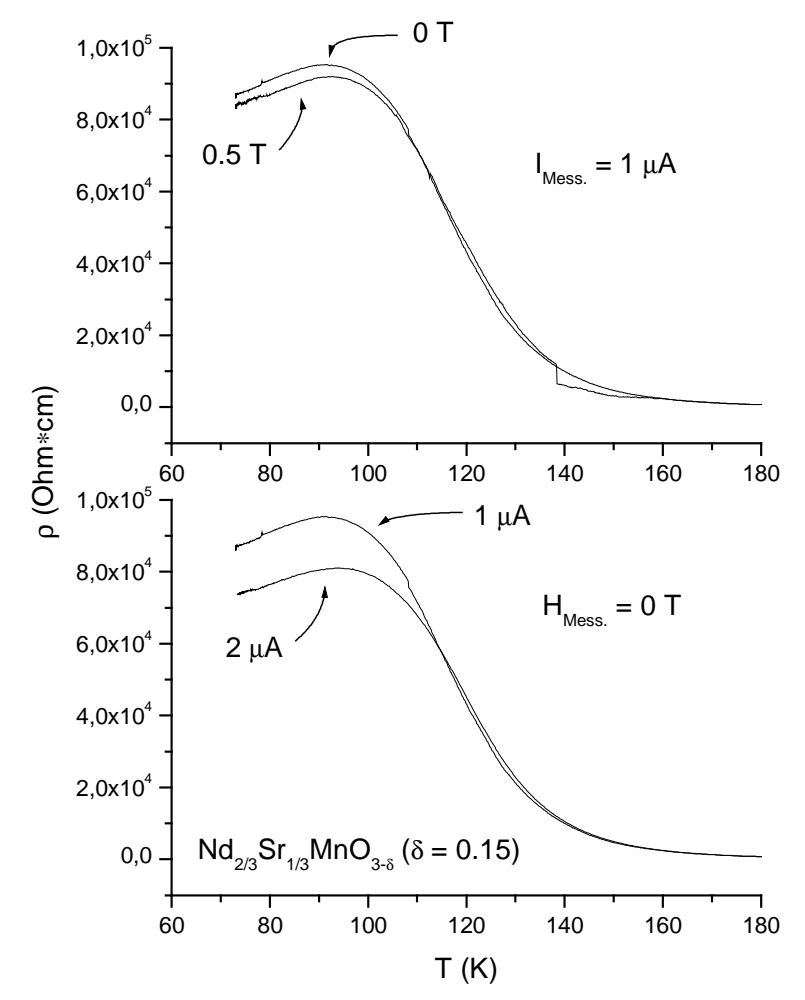

Abbildung 4.24: Zur Demonstration der Äquivalenz zwischen dem magnetoresistiven und elektroresistiven Effekt.

Durch eine Änderung der effektiven Energielücke $\Delta \varepsilon(\mathrm{E}, \mathrm{H}, \mathrm{T})$ kann der Probenwiderstand stark beeinflusst werden, siehe $\rho=\rho_{0} \exp (\Delta \varepsilon / \mathrm{kT})$. In der Abbildung 4.25 wird eine mögliche Temperaturabhängigkeit der Energielücke schematisch gezeigt (durchgezogene Linie). Im elektrischen Feld verschieben sich nun beide Übergangstemperaturen $T_{1}$ und $T_{m i}$. Um die Ergebnisse des Experiments zu erklären, müssen wir eine positive Änderung für $\mathrm{T}_{\mathrm{mi}}$ annehmen $\left(\mathrm{dT}_{\mathrm{mi}} / \mathrm{dE}>0\right)$, was dem Fall $\mathrm{I}$ in Abb. 4.21 entspricht (negative Suszeptibilität der Flachbandzustände, $\left.\Delta \chi_{\text {ud }} *<0\right)$ und gleichzeitig eine Reduktion der effektiven Energielücke $\Delta \varepsilon$ bedeutet. Da stets $\mathrm{dT}_{1} / \mathrm{dE}>0$ ist, verschiebt sich der $\left(\mathrm{T}_{1}, \Delta \varepsilon\right)$-Punkt in Abb. 4.25a nach rechts und nach unten bei einem endlichen Feld $\mathrm{E}_{1}$. Die Suszeptibilität hängt aber von der Temperatur stark ab [15], und zwar nach einem Curie-Gesetz, $\Delta \chi=\Delta \mathrm{C}_{\mathrm{ud}} /\left(\mathrm{T}-\mathrm{T}_{1}(0)\right)$. Dies bedeutet, dass der quadratische feldinduzierte Term bei steigenden Temperaturen verschwindet [103]. Aus diesen Überlegungen folgt, dass das System eine negativen magnetoresitiven Effekt aufweisen sollte, was tatsächlich der Fall ist, wenn das E-Feld genug groß wird (Abb. 3.17 in „Messergebnisse“). Im Experiment ist in der Nähe von $\mathrm{T}_{\mathrm{B}}$ aber ein Temperaturbereich zu finden, in dem der EREffekt bei kleinen Feldern positiv ist (Abb. 4.24, unten). Wenn die anfängliche $(\mathrm{E}=0=\mathrm{H})$ Energielücke $\Delta \varepsilon_{\text {ud }}$ von dem angelegten Feld auch nur leicht abhängig ist, wird die effektive Energielücke bei kleinen Feldern praktisch nur von dieser $\Delta \varepsilon_{\mathrm{ud}}$-̈̈nderung bestimmt. Eine positive $\Delta \varepsilon_{\mathrm{ud}}$-Änderung gibt uns nun die Möglichkeit, den positiven ER-Effekt bei kleinen 
Feldern zu verstehen. Bei höheren Feldern überwiegt dann aber die zusätzliche Änderung der Energielücke durch den feldquadratischen Term.

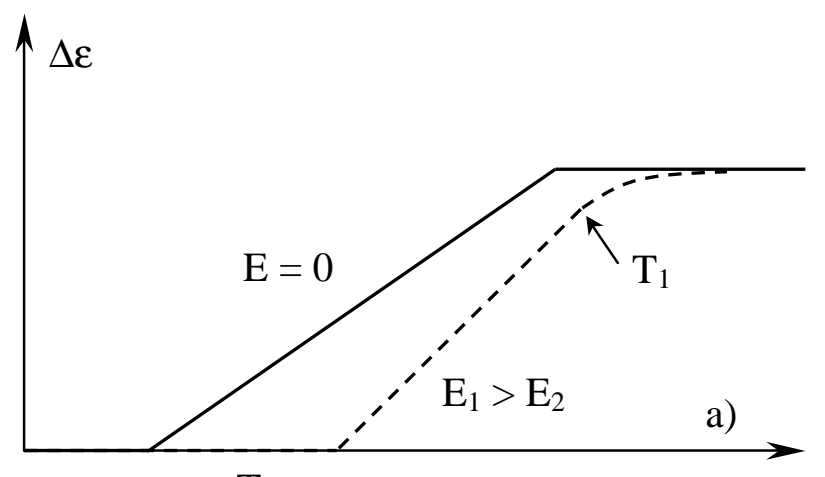

$\mathrm{T}_{\mathrm{mi}}$

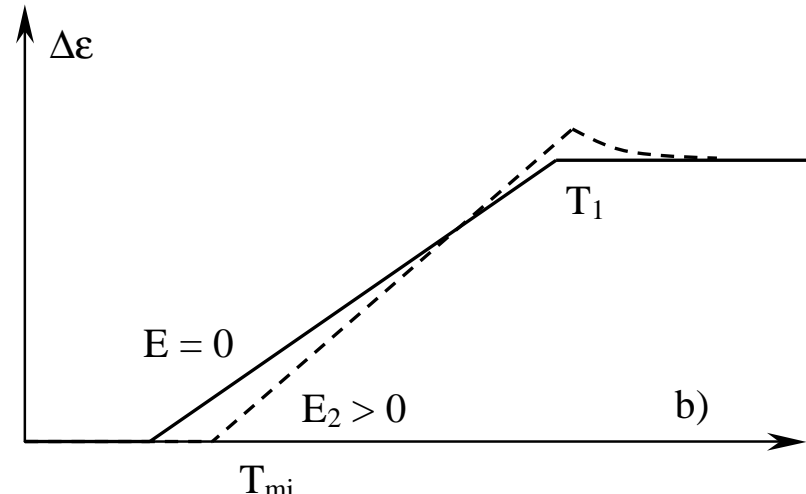

$\mathrm{T}_{\mathrm{mi}}$

Abbildung 4.25: Schematische Darstellung einer möglichen Temperaturabhängigkeit der effektiven Energielücke und ihre Änderung im E- oder H-Feld; a) größere Felder, b) kleine Felder

In der Abbildung 4.24 (oben) wird auch eine Messung des elektrischen Widerstandes der $\mathrm{Nd}_{2 / 3} \mathrm{Sr}_{1 / 3} \mathrm{MnO}_{3-\delta}$-Probe $(\delta=0.15)$ im magnetischen Feld dargestellt. Überraschenderweise stellt man fest, dass das Anlegen des magnetischen und elektrischen Feldes zu einem qualitativ identischen Ergebnis führt: Das System zeigt bei niedrigen Temperaturen einen negativen Magnetowiderstand, der bei Temperaturerhöhung positiv wird. Dies deutet in diesem Fall auf eine Äquivalenz des magnetischen und elektrischen Feldes bezüglich ihrer Wirkung auf die Probeneigenschaften hin, im Gegensatz zu der Situation in den korngrenznahen Bereichen $\left(\mathrm{T}_{\mathrm{K}}\right.$ wird vom E- und H-Feld in verschiedene Richtungen geschoben). Beides ist nach Gl. 4.15 - 4.17 möglich.

Der numerische Vergleich der Stark- und Zeemanenergie bei Abb. 4.24 ergäbe eine ionische Polarisation, d.h. elektrische Dipole wie beim $\mathrm{BaTiO}_{3}$, nur in geringer Anzahldichte.

\section{Spinabhängiger Barrierenwiderstand}

In der Abbildung 4.26a ist die Kennlinie $\mathrm{V}(\mathrm{I})$ einer keramischen $\mathrm{Nd}_{2 / 3} \mathrm{Sr}_{1 / 3} \mathrm{MnO}_{3-\delta}$-Probe $(\delta=$ 0.10 ) gezeigt (gemessen bei einer Temperatur $\mathrm{T}=74 \mathrm{~K}$ ). V(I) ist stark nichtlinear. Eine ähnliche Kennlinie ist auch bei der Probe mit $\delta=0.05 \mathrm{zu}$ finden (siehe Abb. 3.14). Die negative Steigung im Mittelteil der Kennlinie ist im Rahmen des oben diskutierten erweiterten deGennes Modells nicht zu erklären. Wir können diesen Effekt daher nicht dem Volumenbereich zuordnen. Es ist aber bekannt, dass eine parallele Ausrichtung des Magnetisierungsvektors von zwei benachbarten Bereichen (Domäne, Bereiche der homogenen Phase oder Körner) eine Erhöhung der Leitfähigkeit durch die Grenze zwischen diesen Bereichen zur Folge hat [104] (Spinabhängiger Transport durch Tunneln oder Injektion). Dies sieht man z.B. als kontinuierlichen Untergrund in der Messungen des Magnetowiderstandes. Dies wird i.A. einem spinabhängigen Tunnelstrom durch die Korngrenze zugeordnet [105] und ist dem intrinsischen MR-Effekt bei $\mathrm{T}_{\mathrm{C}}$ überlagert (Abb. 4.26b). 

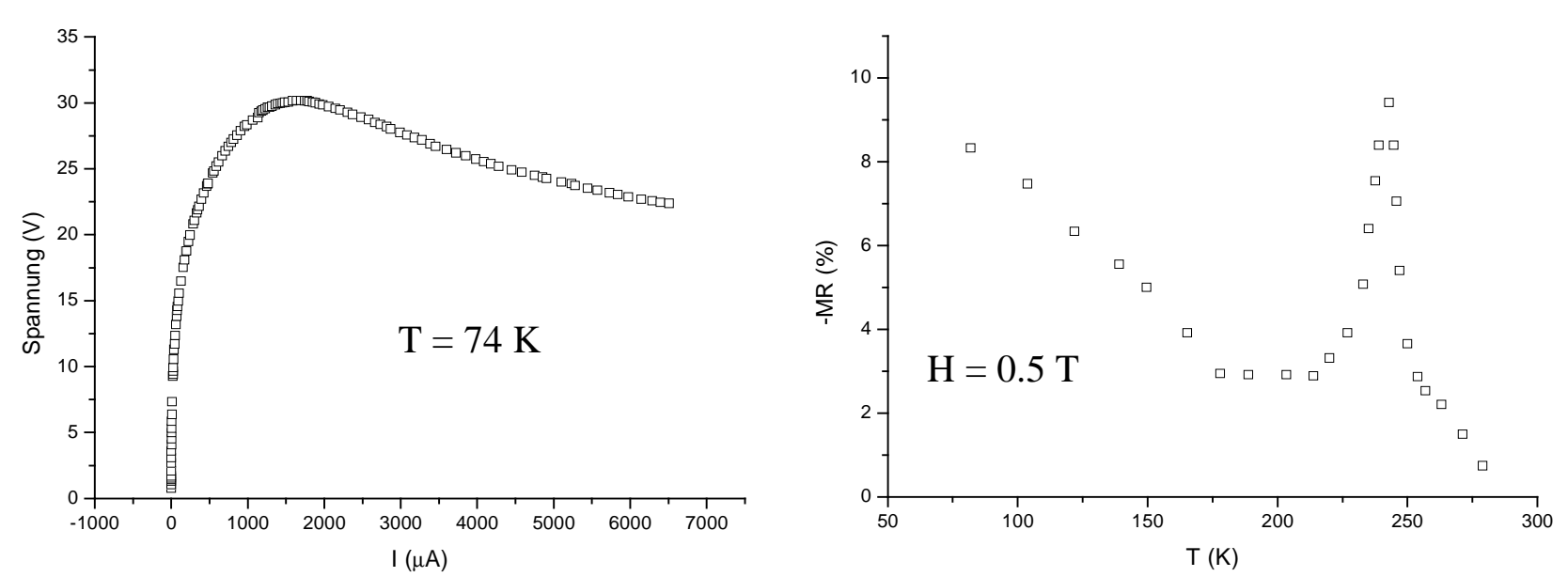

Abbildung 4.26: Tunneln oder Injektion über die Korngrenzen. a) die V(I)-Kennlinie, gemessen an der $\mathrm{Nd}_{2 / 3} \mathrm{Sr}_{1 / 3} \mathrm{MnO}_{2.9}$-Probe; b) Der Magnetowiderstand einer $\mathrm{Nd}_{2 / 3} \mathrm{Sr}_{1 / 3} \mathrm{MnO}_{3}$-Probe. Nach [17].

Um den Barrierenwiderstand mit einem angelegten Magnetfeld überhaupt variieren zu können, müssen die Leitungselektronen polarisiert sein. Auch muss der relative Winkel zwischen den Magnetisierungen der benachbarten Volumenbereichen veränderbar sein, was aber nur dann möglich ist, wenn die Volumenbereiche eine ferromagnetische Komponente aufweisen, d.h. wenn der mittlere Kantungswinkel $\theta_{0}>0$ ist [10]. Tatsächlich werden solche Nichtlinearitäten in den Kennlinien V(I) nur unterhalb der Temperatur $T_{B}$ gefunden, wo wir eine gekantete Struktur vermuten. Damit ein elektroresistiver Barrieren-Effekt eintreten kann, braucht man nur noch eine vektorielle Kopplung zwischen dem Spinsystem und dem System der elektrischen Dipole. 


\section{Zusammenfassung und Ausblick.}

Im Rahmen dieser Arbeit wurden sauerstoffdefizitäre keramische $\mathrm{Nd}_{2 / 3} \mathrm{Sr}_{1 / 3} \mathrm{MnO}_{3-\delta}$-Proben $(0<$ $\delta<0.20)$ untersucht. Wir haben festgestellt, dass die Variation des Sauerstoffdefizits zu einem $\mathrm{T}-\delta$-Phasendiagramm führt, das paramagnetische, antiferromagnetische und zwei magnetisch gekantete (isolierend und metallisch) Bereiche enthält. Dieses Diagramm lässt sich im Rahmen einer erweiterten deGennes-Theorie erklären. Man muss aber bei der theoretischen Beschreibung dieser Systeme die Phasenseparationsphänomene mit einbeziehen. So wird angenommen, dass bei geringen $\delta$-Werten die Phasenseparation eine überwiegende Rolle spielt, was auch mit den zahlreichen Literaturdaten übereinstimmt. Es wird aber hier auch gezeigt, dass die Phasenseparation bei hohem Sauerstoffdefizit unterdrückt wird, womit die gekanteten deGennesPhasen eine führende Rolle übernehmen. Das System bleibt im Zwischenbereich sehr komplex, insbesondere bei mittleren $\delta$-Werten, wo die Phasenseparation und der deGennes-Zustand überlagern. Bei der Diskussion konzentrieren wir uns daher im Wesentlichen auf die linke und die rechte Seite des Phasendiagramms, wo entweder die Kantung oder die Phasenseparationseffekte vernachlässigt werden können.

Es wird insbesondere das Auftreten eines isolierenden gekanteten Zustandes in einem Temperaturbereich $\theta_{\mathrm{f}}<\mathrm{T}<\mathrm{T}_{\mathrm{B}}$ festgestellt. Dieser wird einer halbmetallischen elektronischen Situation zugeordnet. Es wurde auch festgestellt, dass die Ordnungstemperaturen in diesem Bereich vom elektrischen und magnetischen Feld abhängig sind. Die Empfindlichkeit der Proben auf ein inneres E-Feld (elektroresistiver Effekt) lässt die Existenz von elektrischen Dipolen in der Probe vermuten.

Da die Stärke der elektroresistiven Effekte mit $\delta$ zunimmt, verbinden wir das Auftreten der elektrischen Dipole mit einer lokalen Rekonfiguration des Gitters in der unmittelbaren Nachbarschaft der durch den Sauerstoffdefizit eingebrachten Defekte. Die atomare Struktur dieser Defekte ist bis heute noch nicht genügend erforscht. Die Röntgenuntersuchungen zeigen immerhin, dass das Perowskitgitter bis zum hohen formalen Sauerstoffdefizit (20\%) stabil bleibt. Dies deutet darauf hin, dass die Ionenverschiebungen bei solch einer Rekonfiguration sehr klein sind. Hier muss man auf verfeinerte Ergebnisse aus Röntgen- oder Neutronenbeugungsmethoden oder aus lokalen Mikroskopiemethoden warten.

Es ist naheliegend, dass es zwischen dem magnetischen und elektrischen Dipolsystem zu einer Kopplung kommt. Dies lässt schon die Äquivalenz der magnetoresistiven und der elektroresistiven Effekte vermuten.

Zudem wurde eine anomale Änderung des Widerstandes bei einer Temperatur $\mathrm{T}_{K}$ gefunden, die als Metallisierung der den Korngrenzen naheliegenden Volumenereichen erklärt wird. Da dieser Effekt mit den Korngrenzen eng zusammenhängt, wird vermutet, dass sie bei einkristallinen Probe nicht zu finden sind. Tatsächlich sind die ER-Effekte in durch Laserablation hergestellten Proben sehr viel geringer. 
Ein mögliches Modell zur Beschreibung der experimentell beobachteten Schalteffekte ist eine kollektive Metallisierung mehrerer Korngrenzen. Die E-Feld induzierte Verschiebung der Phasengrenzen im erweiterten deGennes-Modell liefert dazu die Basis. Diese Schalteffekte sind aus der Sicht möglicher technischen Anwendungen sehr interessant. Man könnte in diesem Zusammenhang insbesondere versuchen, einzelne Korngrenzen zu isolieren oder ein ähnliches Schichtsystem zu konstruieren.

Das experimentell beobachtete Kaltleiterverhalten der Kennlinien V(I) kann nicht intrinsischen elektroresistiven Effekten zugeordnet werden. Hier muss man einen elektrischen Barrierenwiderstand hinzunehmen. Der Barrierenwiderstand kann hier zusätzlich durch ein magnetisches Feld verändert werden (Spinabhängiger Varistor).

Bei den Manganiten sind bis heute nur zwei Basissysteme gefunden werden, bei denen ausgeprägte ER-Effekte auftreten $\left(\mathrm{Nd}_{2 / 3} \mathrm{Sr}_{1 / 3} \mathrm{MnO}_{3-\delta}\right.$ und $\left.\mathrm{Pr}_{0.7} \mathrm{Ca}_{0.3} \mathrm{MnO}_{3}\right)$. Dies liegt vermutlich daran, dass mehrere Bedingungen erfüllt sein müssen. Davon können wir zwei benennen: 1) die Existenz elektrischer Dipole und 2) eine elektronische Situation, die dem Halbmetall entspricht. Die Entstehung der elektrischen Dipole wird wahrscheinlich auch noch durch eine große Disproportion der mittleren Ionenradien an den Plätzen A und B begünstigt, wie auch beim $\mathrm{BaTiO}_{3}$. 


\section{Anhang A: Widerstandsinstabilitäten}

\section{Elektrische und chemische Situation nahe der Korngrenze.}

Bei verschiedenen Messungen an den $\mathrm{Nd}_{2 / 3} \mathrm{Sr}_{1 / 3} \mathrm{MnO}_{3-\delta}$-Proben haben wir Instabilitäten beobachtet (siehe Abb. 3.17, 3.21, 3.28 und 3.29). Dazu präsentieren wir das folgende Szenario. Es benutzt lediglich die bereits gemachten Annahmen und die Existenz von Korngrenzen. Unsere Proben sind keramische Proben, d.h. sie sind polykristallin. Bei den Schalteffekten spielen daher möglicherweise die Korngrenzen eine Hauptrolle. In der Abbildung A1 wird ein TEM-Bild von einer Probe mit $\delta=0.15$ dargestellt. Da sind insbesondere zwei benachbarte Kristallite zu erkennen. Mit der weißen gestrichelten Linie wurde die Korngrenze ausgezeichnet.
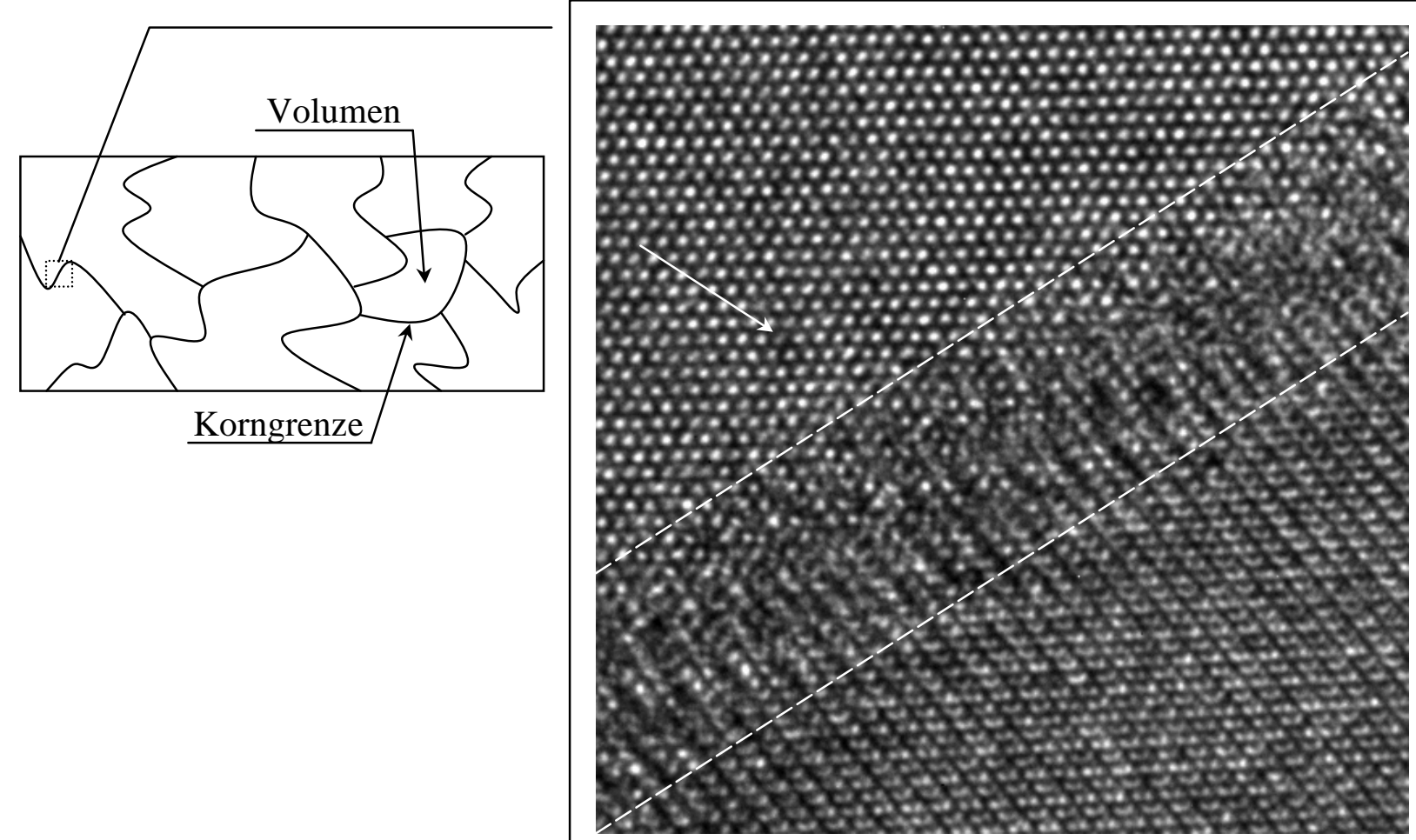

Abbildung A1: Schematische Darstellung der polykristallinen Struktur einer keramischen Probe und das TEM-Bild einer $\mathrm{Nd}_{2 / 3} \mathrm{Sr}_{1 / 3} \mathrm{MnO}_{2.85}$-Probe von der Grenze zwischen zwei benachbarten Kristalliten.

Es ist bekannt, dass in Manganiten der Bereich in der Nähe der Korngrenzen (und an der Oberfläche) immer weniger leitfähig ist als Bereiche weiter außerhalb (innerhalb) [8]. Da ist natürlich die erste Frage, wie weit der Einfluss der Korngrenzen oder der Oberfläche reicht. Wir nehmen an, dass die Korngrenze einen sauerstoffarmen Bereich zwischen zwei Körnern aufbaut, der bis zu $1 \mu \mathrm{m}$ ausgedehnt sein kann. Wenn die Probe von Anfang an als stoichiometrische Probe präpariert wurde, haben die Korngrenzen bereits ein Defizit von Sauerstoff. Der Grund dafür ist, dass die Probe den inneren Sauerstoff vermutlich bevorzugt über die Korngrenze abgibt. Es gibt also immer einen Gradienten von $\delta$ in der Nähe der Korngrenze in Richtung des Zentrums. Auf dem TEM-Bild sind sowohl außerhalb als auch im Inneren des Volumens (Pfeil 
in Abb. A1) dunkle Stellen zu erkennen. Solche Verdunklungen zeigen uns möglicherweise sauerstoffarme Stellen.

Wenn man jetzt eine Spannung an die gesamte Probe anlegt, fällt das Potential hauptsächlich an den Korngrenzenbereichen ab. Im Volumen wird es nur kleinere Potentialänderungen geben, da der spezifische Widerstand des Volumens deutlich kleiner ist. Das wird schon deutlich, wenn man die Resistivität von einkristallinem Manganit mit der von polykristallinem Material sowie von gezielt nichtstoichiometrischem Manganit vergleicht. Als Beispiele wurden die Resistivitätskurven von $\mathrm{La}_{0.85} \mathrm{Sr}_{0.15} \mathrm{MnO}_{3}$-Proben /8/ im keramischen und polykristallinen Zustand und die von sauerstoffdefizitären $\mathrm{Nd}_{0.67} \mathrm{Sr}_{0.33} \mathrm{MnO}_{3-\delta}$-Proben /6/ ausgesucht (Abbildung A2.). In der Abbildung A2a sieht man, dass sich bei Keramik ein sekundäres Resistivitätsmaximum ausbildet. Dieses zweite Maximum wird dem weniger stoichiometrischen Korngrenzenbereich zugeordnet. Vergleichen wir nun zwei keramische Proben aber mit verschiedenem Sauerstoffgehalt (Abbildung A2b) (der $\delta$-Wert wurde hier nach $/ 7 /$ abgeschätzt): man sieht sofort, dass sich mit zunehmendem Sauerstoffdefizit das sekundäre Maximum sehr schnell vergrößert. Das bedeutet aber, dass in der Probe die Korngrenzenbereiche stets einen größeren Widerstand haben als gleichgroßes Domänen-Volumen.
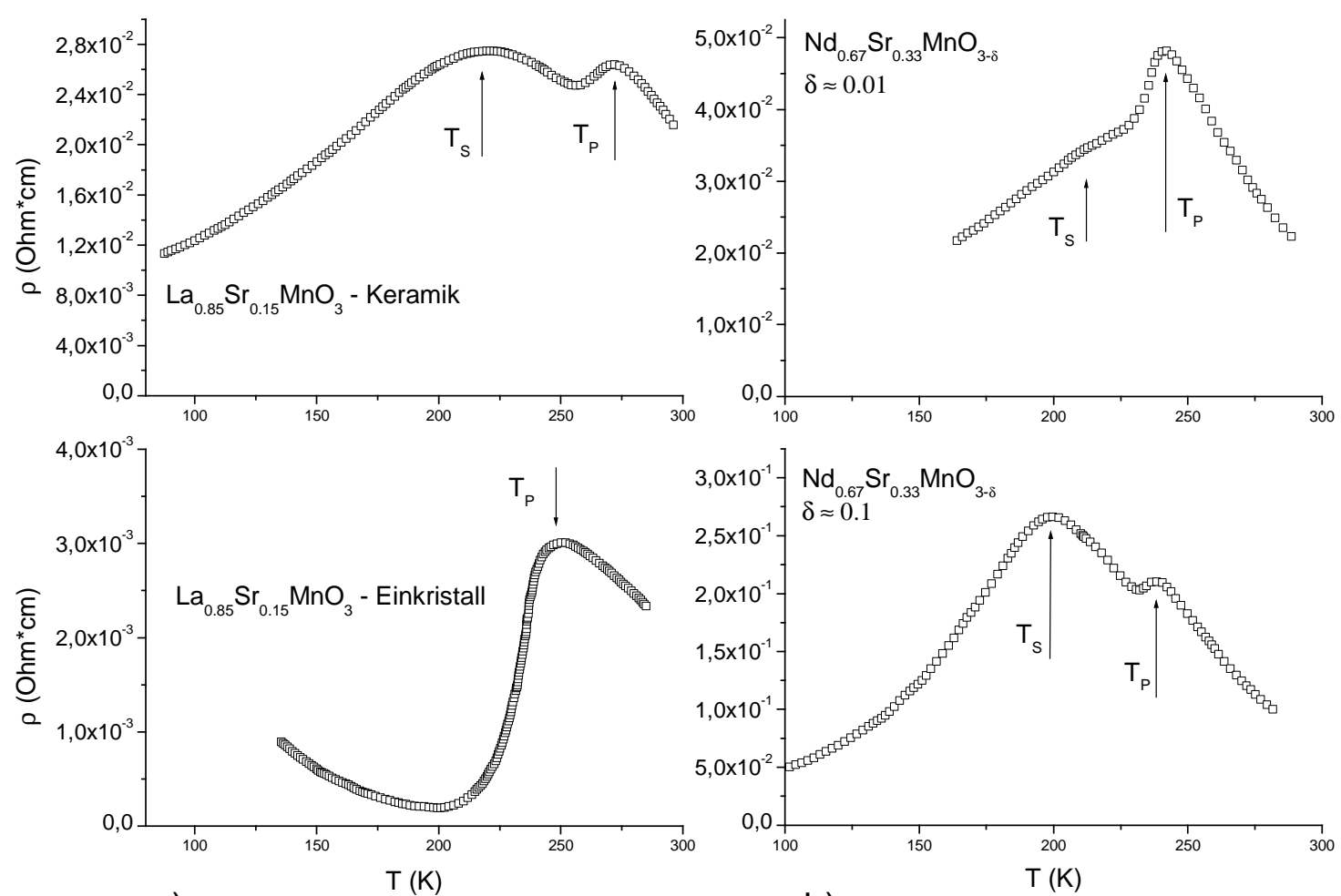

a)

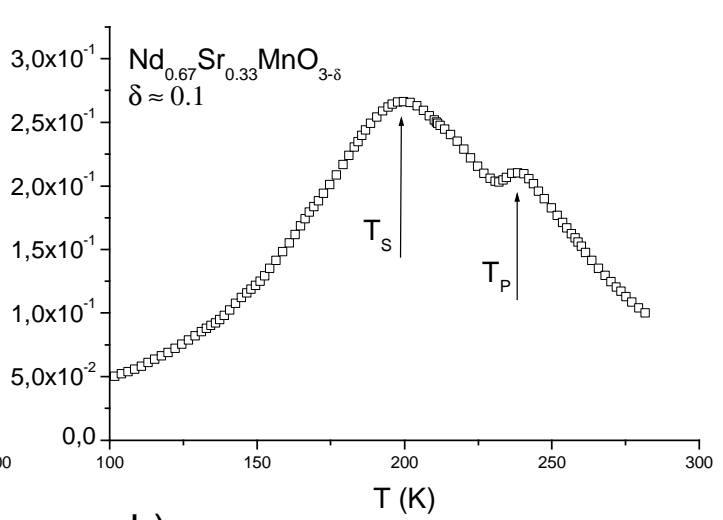

b)

Abbildung A2: Zur Demonstration der Eigenschaften der Korngrenzenbereiche.

a) Spezifischer Widerstand einer keramischen Probe (oben) und eines Einkristalles (unten) mit derselben Zusammensetzung $\left(\mathrm{La}_{0.85} \mathrm{Sr}_{0.15} \mathrm{MnO}_{3}\right)$ [8]; b) Spezifischer Widerstand von zwei keramischen $\mathrm{Nd}_{0.67} \mathrm{Sr}_{0.33} \mathrm{MnO}_{3-\delta}$ Proben mit verschiedenem $\delta[6]$. 
Der Potentialabfall in Korngrenzennähe ist in Abbildung A3a schematisch dargestellt. Die Potentialänderung ist deutlich größer als im Volumen. Entsprechend dem Potentialabfall ist das elektrische Feld $(\mathrm{E}=-\operatorname{grad} \mathrm{U})$ in den weniger leitfähigen sauerstoffarmen Korngrenzenbereichen viel größer als im angrenzenden Volumen.

a)

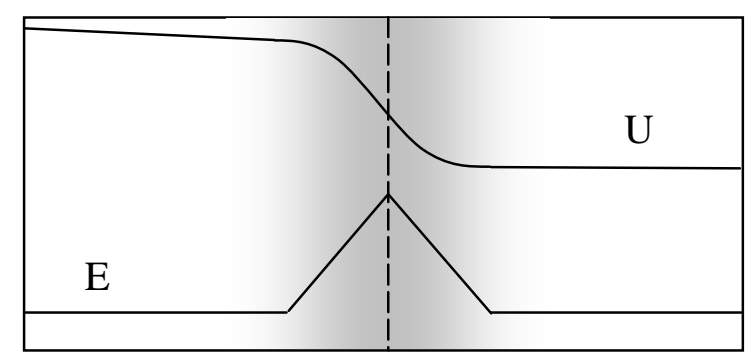

b)

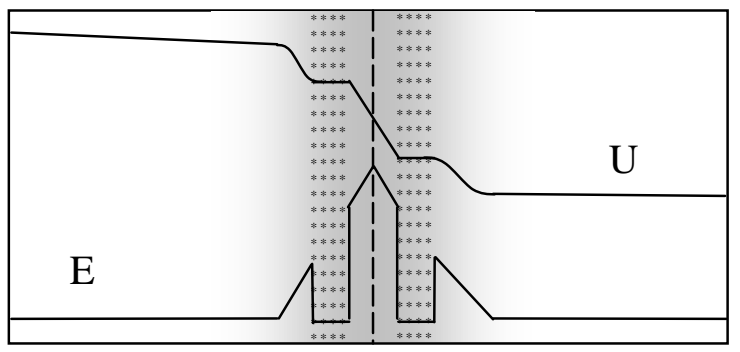

Abbildung A3: a) Korngrenze in einer polykristallinen Probe, Potentialabfall und elektrisches Feld in der Nähe einer Korngrenze im isolierenden und metallisierten Zustand ( $a$ und b). Die Verdunklungen zeigen die nicht stoichiometrischen Bereiche.

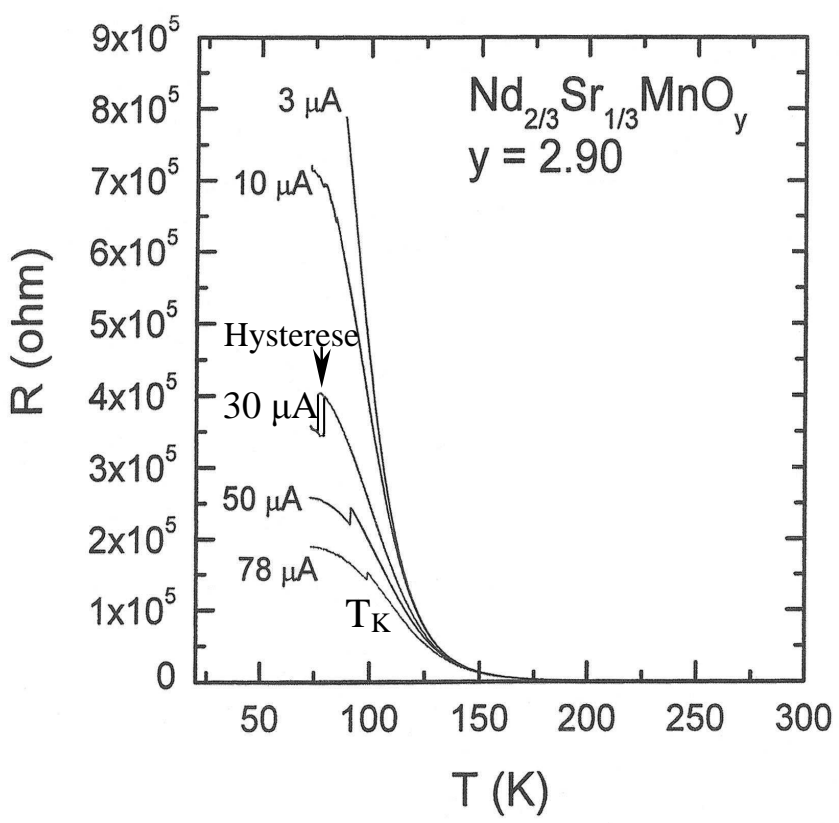

Abbildung A4: Widerstandskurven, die unter konstantem Strom gemessen wurden. Die R(T, I)Kurven bei den größeren Strömen (30 $\mu A$ und größer) zeigen einen Sprung bei einer Temperatur $T_{K}$.

Ist die lokale elektrische Feldstärke genügend groß, so können bei Manganiten die Korngrenzbereiche durch eine E-Feldabhängigkeit der elektronischen Zustände (Stark-Effekt) in einen metallischen (niedrigohmigen) Zustand überspringen [97]. Es kann also zu einem durch ein elektrisches Feld induzierten Phasenübergang zwischen einem metallischen und einem isolierenden Zustand in einem schmalen Bereich an der Korngrenze kommen. Diesen Effekt sieht man in der Tat direkt in den $\mathrm{R}\left(\mathrm{T}\right.$, I)-Kurven bei $\mathrm{Nd}_{2 / 3} \mathrm{Sr}_{1 / 3} \mathrm{MnO}_{3-\delta}$ mit größerem $\delta$ 
(Abbildung A4). Bei kleineren angelegten Potentialen U (nach $\mathrm{I}=\mathrm{UR}$ ) gibt es noch keinen Phasenübergang. Bei größeren Strömen, wo dann auch die E-Feldstärke genügend groß ist, findet man bei einer bestimmten Temperatur $\mathrm{T}_{\mathrm{K}}$ einen Sprung mit Hysterese. Beides ist typisch für einen Phasenübergang. Der Potentialabfall und das entsprechende elektrische Feld für diesen Fall sind in der Abbildung A3b skizziert worden. Bei der Metallisierung wird das ursprünglich erzeugende E-Feld und der eventuell im isolierenden Zustand induzierte ionische Dipol wieder geschwächt (Abschirmung). Unter bestimmten Bedingungen könnte die Probe dann sogar in den isolierenden Zustand zurückspringen. Dann gäbe es in der Probe eine Relaxationsschwingung zwischen den beiden Zuständen, metallisiert und isolierend.

Da eine beliebig ausgewählte Probe sicherlich mehrere Korngrenzen enthält, erhält man sofort ein sehr kompliziertes elektrisch rückgekoppeltes System. Um das Verhalten von solchen Systemen zu untersuchen, wäre eine Computersimulation der beste Weg.

\section{Rauschanalyse}

Oft beobachten wir ungewöhnliche Schwankungen in der R(T)-Kurve, die aber nicht einem Kontaktrauschen zugeordnet werden können. Ähnliche Effekte sind auch in [9] beschrieben worden. Bei unseren digital erfassten R(T)-Messungen gibt es einige, bei denen der Widerstand in einem bestimmten Temperaturbereich fast konstant bleibt. Die Messungen sind mit einer sehr kleinen Aufheizrate $\mathrm{dT} / \mathrm{dt} \approx 0.2 \mathrm{~K} / \mathrm{s}$ aufgenommen, so dass eine Messung von $\mathrm{R}$ bei fester Temperatur über ein Zeitintervall dt äquivalent zu einer Messung von $\mathrm{R}$ über ein bestimmtes Temperaturintervall dT sein sollte. Ein Beispiel ist in der Abbildung A5 zu sehen. Die R(T)Kurve (bei $10 \mathrm{~mA}$ gemessen) ist im Bereich von $110 \mathrm{~K}$ und tiefer fast konstant. Sind $\Delta \mathrm{T}$ und $\Delta \mathrm{t}$ äquivalent, kann man nun einen kleinen Ausschnitt aus dieser Messung für eine Fourier-Analyse des Rauschens benutzen. Wir haben den Bereich zwischen 94 und $98 \mathrm{~K}$ für die Analyse ausgewählt. Zwischen jedem Messpunkt liegt eine Sekunde. Diese Information gibt uns eine Möglichkeit, den Frequenzbereich einzugrenzen.

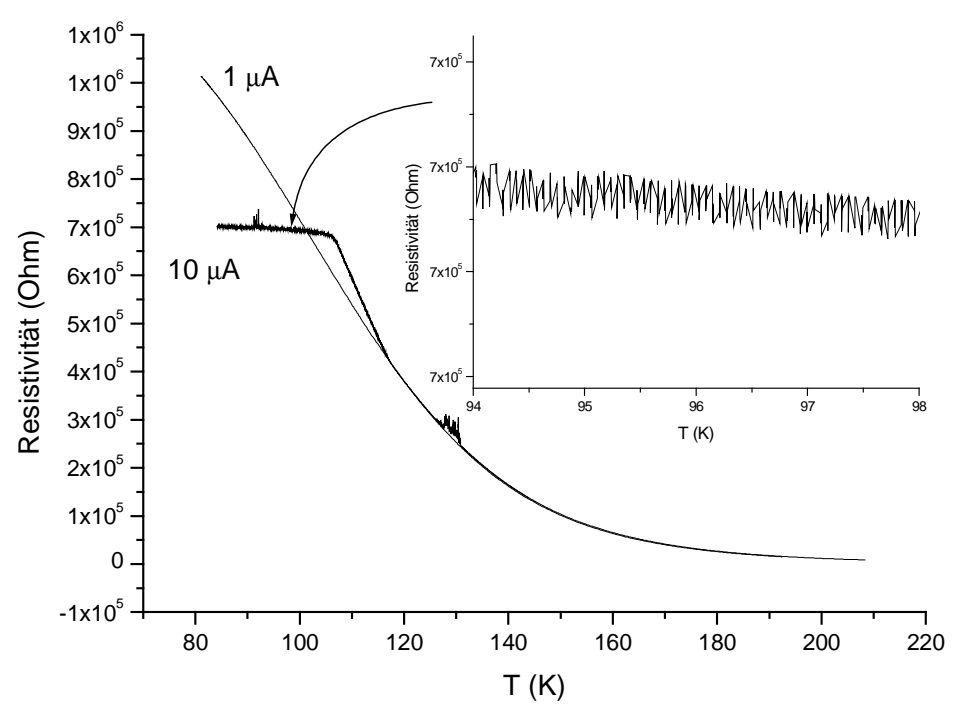

Abbildung A5: Widerstand einer keramischen $\mathrm{Nd}_{2 / 3} \mathrm{Sr}_{1 / 3} \mathrm{MnO}_{3-\delta}$ Probe. Der vergrößerte Ausschnitt (von 94 bis $98 \mathrm{~K}$ ) wurde für die Rauschanalyse verwendet. 
In [54] werden dazu innere Oszillationen in Korngrenzennahe diskutiert! Die Fourier-Analyse eines simulierten Signals wird in Abb. A6 ebenfalls gezeigt. Die Linie mit der Frequenz $0 \mathrm{~Hz}$ wurde in beiden Fällen nicht dargestellt.

Erstaunlicherweise findet man eine Vielzahl von diskreten Schwingungen sowohl im Experiment als auch in der Simulation [54]. Im Detail gibt es aber Differenzen. Man sieht, dass das Spektrum des künstlichen Signals zusätzliche Spitzen enhält. Insgesamt wurden fünf solcher Spitzen mit verschiedenen Amplituden (bei den Frequenzen 1.02, 2.03, 3.06, 4.07 und 8.15 Hz) gefunden. In der Abbildung A6a werden davon nur zwei gezeigt. Das experimentelle Spektrum liefert fast dasselbe Ergebnis (Abbildung A6b). Dem Spektrum ist jedoch ein für $\mathrm{f} \rightarrow 0$ zunehmender Untergrund überlagert, der auf ein Fourier-Integral hinweist. Möglicherweise handelt es sich hier um die Sprünge, die in der Abbildung 4.16 schon gezeigt wurden.
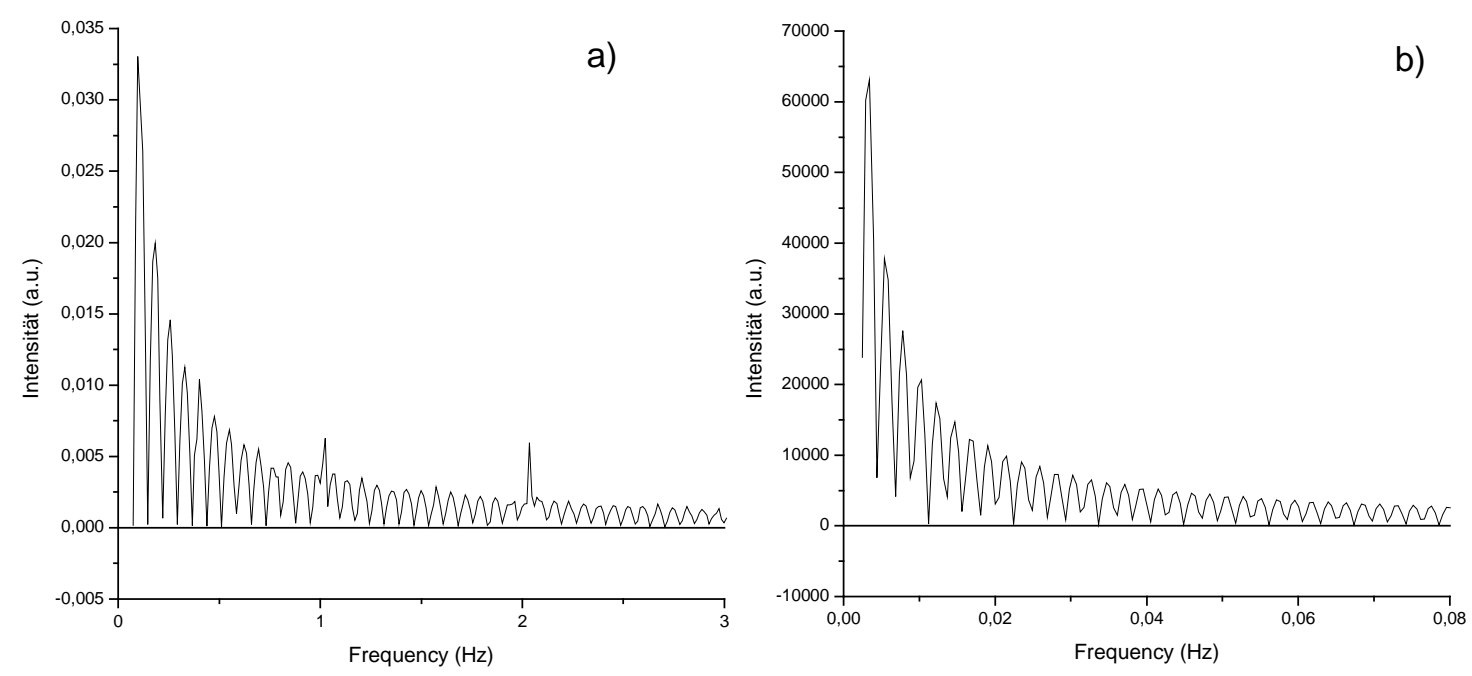

Abbildung A6: a) Spektrum eines künstlichen Signals (siehe [54]), das mit Hilfe einer Computersimulation gewonnen ist; b) Das Spektrum des Widerstandsrauschens einer realen Probe (siehe Abb. A5). 


\section{Anhang B: Doppelwechselfeldmethode zur Bestimmung des barischen Koeffizienten.}

Manganitproben neigen zu einem Zerfall in zwei verschiedene elektronisch-magnetische Phasen. Diese Phasenseparationsphänomene haben auch perkolative Eigenschaften. Für das $\mathrm{Nd}_{2 / 3} \mathrm{Sr}_{1 / 3} \mathrm{MnO}_{3}$-System wird in der Literatur ein Zerfall in die paramagnetischen und ferromagnetishen Bereiche in der Nähe von $T_{C}$ diskutiert, (siehe „Diskussion“, Seite 56). Nach der Phasenseparationstheorie muss die Probe bei Temperaturänderung durch zwei Perkolationsschwellen übergehen. Bei einer Temperatur $\mathrm{T}_{\mathrm{fm}}$ perkolieren die metallischen ferromagnetischen Bereiche, die von einer isolierenden pm-Matrix umgeben sind. Bei einer anderen, tieferen Temperatur $\mathrm{T}_{\mathrm{pm}}$ sollte eine inverse Perkolation stattfinden, wobei in einer metallischen fm-Matrix die isolierenden pm-Bereiche perkolieren. Beide Perkolationsschwellen sind von äußeren Kräften (z.B. Magnetfeld oder hydrostatischer Druck) abhängig [89]. Dies macht es möglich, diese Effekte mit Hilfe von verschiedenen Methoden zu untersuchen, z.B. durch die Detektion der Widerstandsänderung unter äußeren Kräften.

\section{Doppelwechselfeldmethode}

Wir stellen hier eine Methode zur Identifizierung solcher Phasengemische vor. Sie basiert auf der differentiellen Messung des barischen Koeffizienten $\gamma$. Hat man eine Probe, deren Widerstand vom angelegten Druck abhängt, so kann man für den Probenwiderstand bei einer bestimmten Temperatur schreiben:

$\mathrm{R}(\mathrm{p})=\mathrm{R}_{0}(1+\gamma \mathrm{p})$

wobei $\mathrm{R}_{0}$ der unter 0 kbar gemessene Probenwiderstand, $\mathrm{p}$ der angelegte (hydrostatische) Druck und $\gamma$ der sogenannte barische Koeffizient ist. Der $\gamma$-Koeffizient ist eine Größe, die die Widerstandsempfindlichkeit der Probe vom Druck beschreibt, und im Allgemeinen vom Druck selbst abhängig ist. Gleichung (1) kann man auch als den ersten Term einer Reihenentwicklung betrachten. Höhere Terme müssen aber nur in seltenen Fälle berücksichtigt werden.

Mit der hier präsentierten Doppelwechselfeldmethode wurde außer der Druckabhängigkeit des Widerstandes von Manganin der $\gamma$-Koeffizient einer keramischen $\mathrm{Nd}_{2 / 3} \mathrm{Sr}_{1 / 3} \mathrm{MnO}_{3}$-Probe im Temperaturbereich von 194K (Trockeneistemperatur) bis zur Raumtemperatur untersucht. Diese Manganitprobe hat einen großen $\gamma$-Wert und wurde zunächst allein deshalb untersucht.

Wenn der durch die Probe fließende Strom und der an die Probe angelegte Druck periodische Funktionen der Zeit sind, aber mit verschiedenen Frequenzen $\left(\omega_{1}\right.$ und $\left.\omega_{2}\right)$, so kann man eine solche Probe auf Grund des Ohm'schen Gesetzes U = R(p)I als Frequenzmischer benutzen. Das Produkt von zwei periodischen Größen $\left(R=R_{0} \sin \left(\omega_{1} t\right)\right.$ und $I=I_{0} \sin \left(\omega_{2} t\right)$ enthält zwei Seitenbänder $\left(\left(\omega_{1}+\omega_{2}\right)\right.$ und $\left.\left(\omega_{1}-\omega_{2}\right)\right)$. Beide Signale tragen dabei die volle Information über das Produkt und können unabhängig voneinander als detektiertes Signal dienen. Mit Hilfe der frequenzselektiven phasenempfindlichen Gleichrichtung kann man nun Störsignale ausschalten, deren Frequenzen nicht gleich der Summen- oder der Differenzfrequenz sind. So wird die 
Empfindlichkeit der Messungen deutlich erhöht. Nach diesem Prinzip ist unsere Methode entwickelt worden.

\section{Aufbau des Experimentes}

Der experimentelle Aufbau wird in Abbildung B1 dargestellt. Die Probe wurde zusammen mit einer Piezokeramik PIC 151 (Fa. PICeramic) in einer hydrostatischen Druckzelle untergebracht. Die PIC 151 ist eine Keramik, die eine sehr hohe piezoelektrische Konstante hat, und wurde im Form eines Hohlzylinders ausgefertigt. Die Druckzelle wurde mit einer speziellen Flüssigkeit (electronic liquid FC-75) gefüllt. Leitfähigkeit und Kompressibilität von FC-75 sind sehr niedrig. Mit einer an die Piezokeramik angelegten Wechselspannung von $50 \mathrm{~V}$ wurde in der Zelle ein Wechseldruck von $\sim 1 \mu$ bar erzeugt.

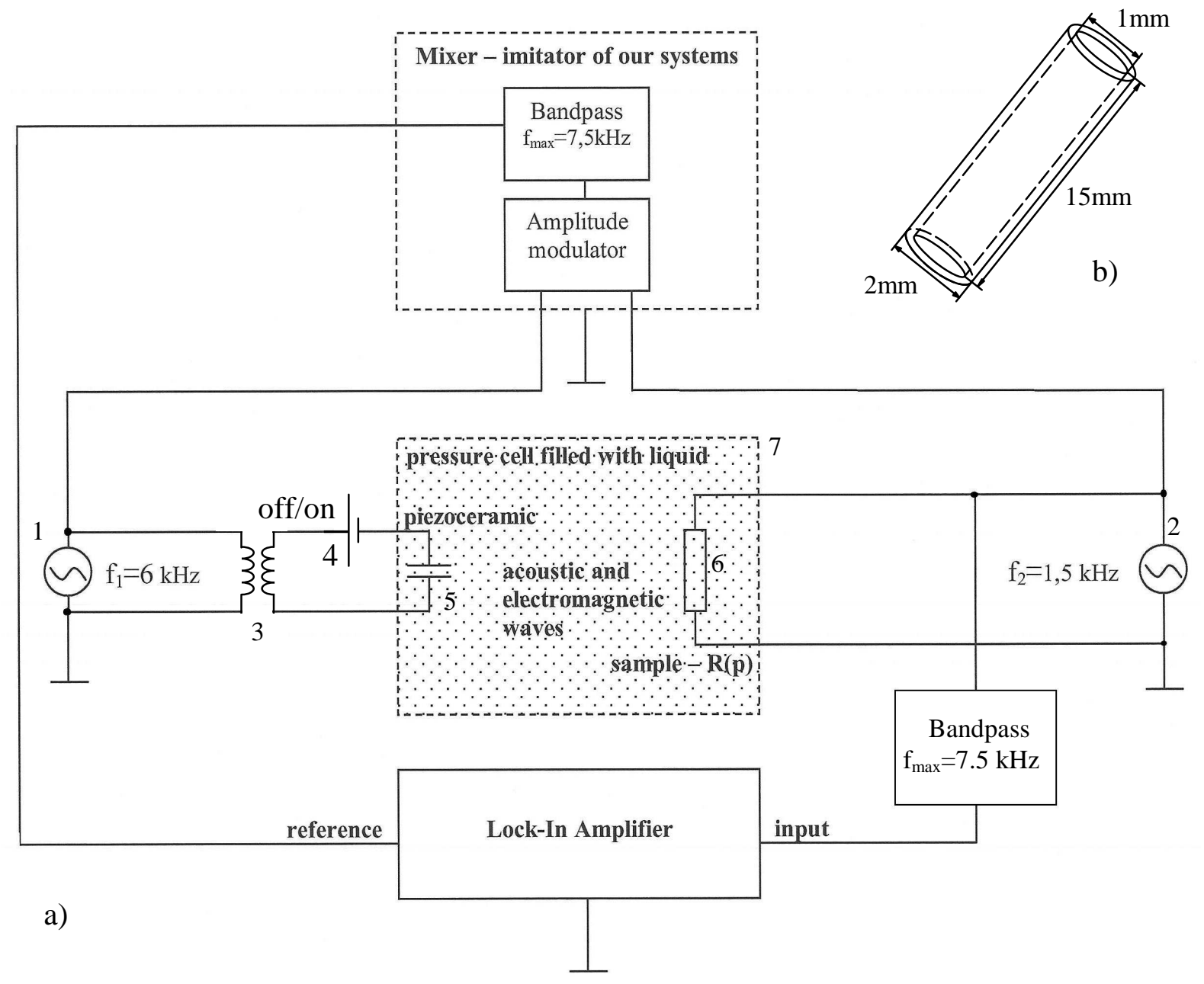
1. Wechselstromquelle $\left(f_{1}=6 \mathrm{kHz}\right)$
5. Piezokeramik (PIC 151)
2. Wechselstromquelle $\left(f_{2}=1.5 \mathrm{kHz}\right)$
6. Probe
3. Transformator
7. Hydrostatische Druckzelle
4. Computergesteuerte Gleichspannungsquelle

Abbildung B1: a) Experimenteller Aufbau zur Messung des barischen Koeffizienten mit Hilfe der Doppelwechselfeldmethode, b) Geometrie der Piezokeramik. 
Der Druck in der Zelle besteht eigentlich aus zwei Komponenten:

$\mathrm{p}=\mathrm{p}_{\mathrm{st}}+\mathrm{p}_{\text {piezo }}$,

wobei $\mathrm{p}_{\mathrm{st}}$ der statische Offset-Druck in der Zelle ist. Dieser Druck wird einmalig für die gesamte Messung eingestellt. $p_{\text {piezo }}$ ist der von der Piezokeramik erzeugte Wechseldruck. In dieser Arbeit werden Messungen unter einem Offset-Druck von 0, 1 und 2 kbar vorgestellt. Da statische $\rho(\mathrm{p}, \mathrm{T})$-Messungen vorlagen [3], dient dies zur zusätzlichen Überprüfung der Eignung der neuen Methode.

Für die Bestimmung der Wechseldruckamplitude $\mathrm{p}_{\sim}$ in der Druckzelle wurde eine Wicklung aus Manganindraht $(11 \% \mathrm{Mn}, 3 \% \mathrm{Ni}, 86 \% \mathrm{Cu})$ benutzt. Diese Legierung wird, dank der Stabilität seines $\gamma$-Koeffizienten $\left(\gamma=2.47 * 10^{-3} \mathrm{kbar}^{-1}\right)$, üblicherweise als Druckdetektor verwendet. Der am Manganindraht gemessene Wert der Wechseldruckamplitude $\mathrm{p}_{\sim}$ hängt natürlich von der Spannungsamplitude des anregenden Signals ab. Bei $U=50 \mathrm{~V}$ betrug er $\sim 1 \mu$ bar. Während des Experimentes wurde der überlagerte hydrostatische Druck ebenfalls mit Hilfe des Manganindrahtes gemessen. Um die Piezokeramik anzuregen und gleichzeitig die Probe mit Wechselstrom zu belasten, sind zwei Generatoren notwendig. Um später eine stabile Referenzfrequenz für den phasenempfindlichen Gleichrichter (5208 Two Phase Lock-In Analyser von EG\&G Brookdeal) zu erzeugen, braucht man zwei Generatoren, deren Phasen starr verbunden sind, d.h. deren Phasendifferenz unveränderlich bleibt. In unserem Fall wurde ein Mutter-Generator benutzt, der rechteckigen Impulse mit der Frequenz 6 kHz abgibt (Ausgang 1) (sehe Abbildung B2). Das Signal wird auch zweimal bis zu $1.5 \mathrm{kHz}$ heruntergeteilt (Ausgang 2). Die Fourier-Komponenten der zwei rechteckige Signale $(6 \mathrm{kHz}$ und $1.5 \mathrm{kHz})$ wurden mit Hilfe scharf eingestellter Bandpass-Filter abgeschnitten.

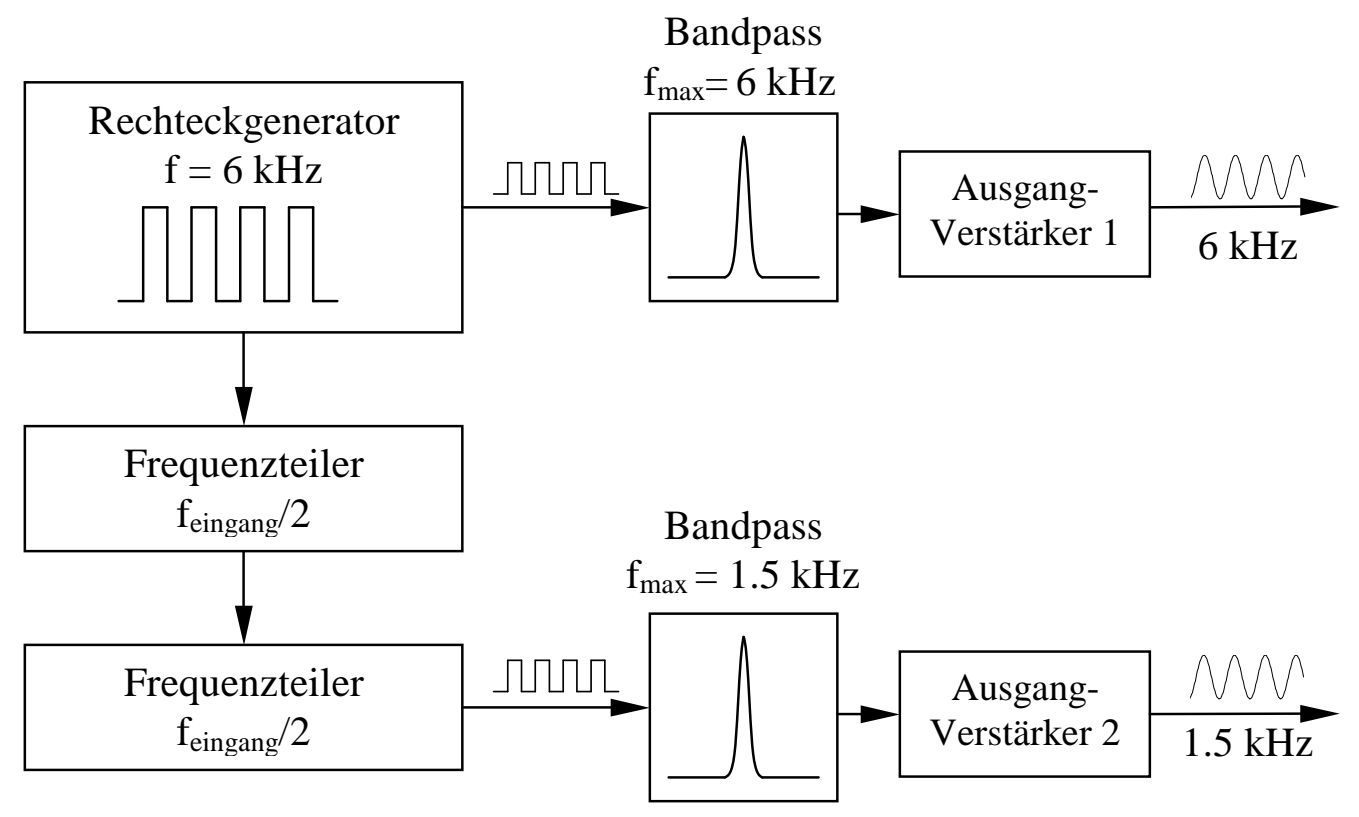

Abbildung B2: Aufbau zur Generation von zwei Wechselspannungen, deren Phasen starr verbunden sind. 
Die Piezokeramik wurde mit dem Signal $f_{1}=6 \mathrm{kHz}$ angeregt; mit dem anderen Signal $\mathrm{f}_{2}=1.5$ $\mathrm{kHz}$ wurde die Probe mit Strom belastet. An dieser Stelle muss man beachten, dass die Piezokeramik nicht nur akustische Wellen ausstrahlt. Da die metallbedampfte Piezokeramik gleichzeitig ein Kondensator ist (die gemessene Kapazität beträgt $\approx 10 \mathrm{pF}$ ), strahlt sie auch hier unerwünschte elektromagnetischen Wellen aus. Zusätzlich ist die Piezokeramik ein Dielektrikum, dessen Polarisierbarkeit $\mathrm{P}(\mathrm{E})$ im elektrischen Feld wegen der Sättigung von $\mathrm{P}$ nicht linear ist $\left(\mathrm{P}=\alpha \mathrm{E}+\beta \mathrm{E}^{2}+\ldots\right)$. In diesem Fall ist die ausgestrahlte EM-Welle eine periodische Funktion, die verschiedene Frequenzen enthält, obwohl die Anregung monofrequent ist. Die Fourierreihe besteht aus einer unendlichen Anzahl von Harmonischen, deren Frequenzen die Mehrfachfrequenzen der Hauptfrequenz sind.

Zusätzlich induziert die Zuleitung zur Piezokeramik, d.h. genauer der Teil, der sich innerhalb der Zelle befindet, an der Probe eine zusätzliche parasitäre induktive Spannung $U_{\text {ind }}$. Diese Spannung $U_{\text {ind }}$ ist immer mehrere Größenordnungen größer als das Signal, das von der Widerstandsmodulation hervorgerufen wird. Würde man nun versuchen, die Widerstandsänderung mit eingeprägtem Gleichstrom zu detektieren, so würde man scheitern, weil das gewünschte Signal und das induzierte Signal dieselbe Frequenz hätten. Eine elegante Lösung dieses Problems ist, das gesuchte Signal in einen anderen Frequenzbereich zu bringen. Das gelingt dadurch, dass man die Doppelwechselfeldmethode einsetzt.

Die gesamte Spannung an der Probe ist:

$\mathrm{U}=\mathrm{IR}+\mathrm{U}_{\text {ind }}$

wobei R nach (1) mit dem Druck in Beziehung steht und I der Wechselstrom mit einer Kreisfrequenz $\omega_{2}$ und einer Amplitude $I_{\sim}$ ist:

$\mathrm{I}=\mathrm{I}_{\sim} \sin \left(\omega_{2} \mathrm{t}\right)$.

Der Druck in der Zelle ist auch eine von der Zeit abhängige Funktion, die aus zwei Komponenten besteht (statische und dynamische):

$\mathrm{p}=\mathrm{p}_{\sim} \sin \left(\omega_{1} \mathrm{t}\right)+\mathrm{p}_{\mathrm{st}}$

hier ist $\mathrm{p}_{\sim}$ die Druckschwingungsamplitude und $\omega_{1}$ die Schwingungsfrequenz. Wir können jetzt die Formel (1) für den Probenwiderstand umschreiben:

$R(p)=R_{0}\left[1+\gamma p_{\sim} \sin \left(\omega_{1} t\right)+\gamma p_{s t}\right]$ oder $R=R_{0}+\gamma p_{s t} R_{0}+\gamma p_{\sim} R_{0} \sin \left(\omega_{1} t\right)$

Setzen wir nun (b6) in Formel (b3), so ergibt sich für die gemessene Spannung insgesamt:

$\mathrm{U}=\mathrm{IR}_{0}+I \gamma \mathrm{p}_{\mathrm{st}} \mathrm{R}_{0}+\mathrm{IR} \mathrm{R}_{0} \gamma \mathrm{p}_{\sim} \sin \left(\omega_{1} \mathrm{t}\right)+\mathrm{U}_{\text {ind }}$, 
Man sieht, dass die Spannung an der Probe die Summe mehrerer Spannungen ist. Der erste und der zweite Term sind Wechselspannungen mit der Frequenz $f_{1}=1.5 \mathrm{kHz}$. Der Term $U_{\text {ind }}$ ist eine induzierte unerwünschte Spannung $\left(U_{\text {ind }}\right.$ ist eine Summe von vielen Signalen mit verschiedenen Frequenzen). Nur der dritte Term enthält das gewünschte Signal:

$\mathrm{U}_{3}=\gamma \mathrm{R}_{0} \mathrm{p}_{\sim} \sin \left(\omega_{1} \mathrm{t}\right) \mathrm{I}_{\sim} \sin \left(\omega_{2} \mathrm{t}\right)=0.5 \gamma \mathrm{p}_{\sim} \mathrm{R}_{0} \mathrm{I}_{\sim}\left[\cos \left(\left(\omega_{1}-\omega_{2}\right) \mathrm{t}\right)-\cos \left(\left(\omega_{1}+\omega_{2}\right) \mathrm{t}\right)\right]$

Dabei wurde für den Wechselstrom I Gl. b4 eingesetzt.

In unserem Aufbau wird nur die zweite Harmonische mit der Frequenz $\omega=\omega_{1}+\omega_{2}$ selektiert:

$\mathrm{U}_{\text {mess }}=0.5 \gamma \mathrm{p}_{\sim} \mathrm{R}_{0} \mathrm{I}_{\sim} \sin \left(\left(\omega_{1}+\omega_{2}\right) \mathrm{t}\right)$.

Alle anderen Frequenzen wurden mit einem Bandpass-Filter ausgefiltert. Um nachfolgend das Signal zu detektieren, wurde die frequenzselektive phasenempfindliche Gleichrichtung verwendet. Mit dem phasenempfindlichen Gleichrichter kann man eine Signalauflösung bis zu einem Nanovolt erreichen.

Um das notwendige stabile Referenzsignal für den Betrieb des Lock-In-Amplifiers zu generieren, muss dessen Frequenz und Phase mit der Frequenz und der Phase des gemessenen Signals übereinstimmen. Dazu haben wir parallel zur Messzelle einen Amplitudenmodulator gebaut. In diesem Modulator werden die zwei Signale ebenfalls von den beiden phasenstarren Generatoren $\left(\mathrm{f}_{1}=6 \mathrm{kHz}\right.$ und $\mathrm{f}_{2}=1.5 \mathrm{kHz}$ ) abgenommen und zusammengemischt. Das garantiert eine konstante Phasendifferenz. Nachfolgend werden alle Signale mit Frequenzen außer der Summenfrequenz $\mathrm{f}_{1}+\mathrm{f}_{2}=7.5 \mathrm{kHz}$ wieder mit Hilfe eines Bandpass-Filters herausgenommen.

Obwohl man mit der frequenzselektiven phasenempfindlichen Gleichrichtung ein gutes SignalRausch-Verhältnis erreicht, war unseres Signal so klein, dass wir es ohne anschließende statistische Mittelung nicht auflösen konnten. Mit einer Computersteuerung war es möglich, bei einer festen Temperatur genügend viele Messpunkte zu sammeln und dann den Mittelwert zu bilden. Durch dieses Verfahren konnten wir Signale im Picovoltbereich auflösen.

Bei der Computererfassung der Rohdaten wurde folgendermaßen vorgegangen. In unserem Experiment wurde die nach Formel (b9) bestimmte Spannung $U_{\text {mess }}$ mit der Frequenz $7.5 \mathrm{kHz}$ durch den Lock-In-Verstärker gemessen. Wenn die Spannung $U_{\text {mess, }}$ die Wechselstromamplitude und der Probenwiderstand bei 0 kbar bekannt sind, kann man den $\gamma$-Koeffizienten ausrechnen. Oft driftete jedoch die Messanzeige im Minutenbereich. Für die Lösung dieses Problems war es entscheidend, das folgende Verfahren einzusetzen: im Lauf des Experiments (bei einer festen Temperatur) wurden Signale $U_{\text {mess }}$ unter zwei verschiedenen Bedingungen aufgenommen $\left(\mathrm{U}^{\mathrm{a}}{ }_{\text {mess }}\right.$ und $\mathrm{U}^{\mathrm{b}}{ }_{\text {mess }}$ ). Das erste Signal wurde aufgenommen, während die Piezokeramik mit der Wechselspannung und einen zusätzlichen Gleichspannungsoffset angeregt wurde (Regime a). Der Offset wurde dabei genau so groß wie die Wechselspannungsamplitude eingestellt. Das 
zweite Signal wurde ohne diesen zusätzlichen Gleichspannungsoffset aufgenommen (Regime b). Dabei erwartet man, dass im ersten Fall der Wechseldruck in der Zelle $\mathrm{p}_{\sim}$ mit einer Frequenz schwingt, die gleich der Anregungsfrequenz ist (siehe Abbildung B3a). Im zweiten Fall wird $\mathrm{p}_{\text {piezo }}$ gleichgerichtet, d.h. hängt von der Zeit ab, wie $|\sin (\omega t)|$ (Abbildung B3b). Das kann man aus der Volumenänderung der Piezokeramik erklären. $\Delta \mathrm{V}$ ist immer positiv, d.h. hängt nicht von der Polarität des anregenden Signals, sondern nur von dessen Betrag ( $\left.\Delta \mathrm{V} \sim \mid \mathrm{U}_{\text {anregung }}\right)$ ab [53]. Diese beide Einstellungen konnten experimentell leicht realisiert werden, ohne dass zusätzliche Fourier-Komponenten in den Messkreis eingestreut wurden.
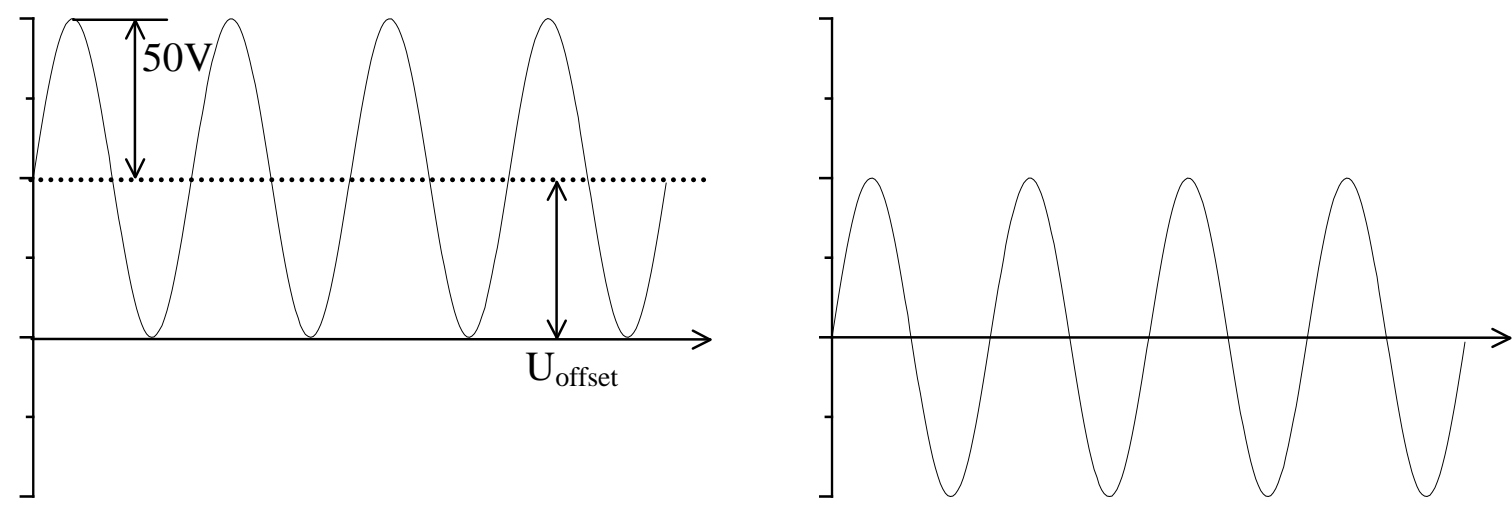

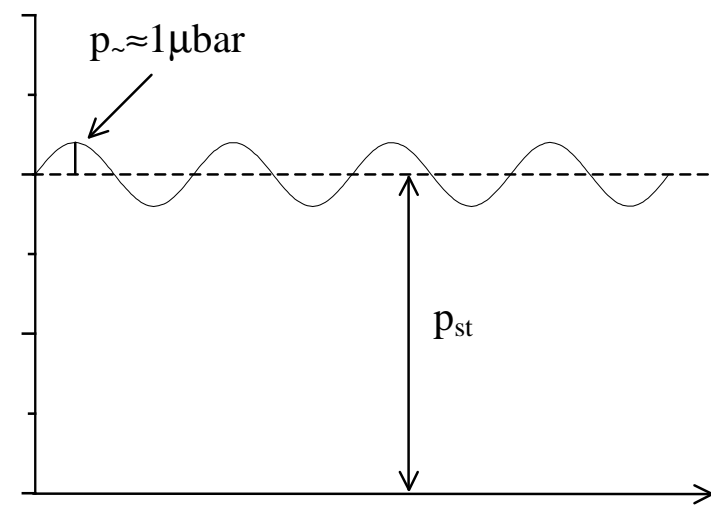

Regime a

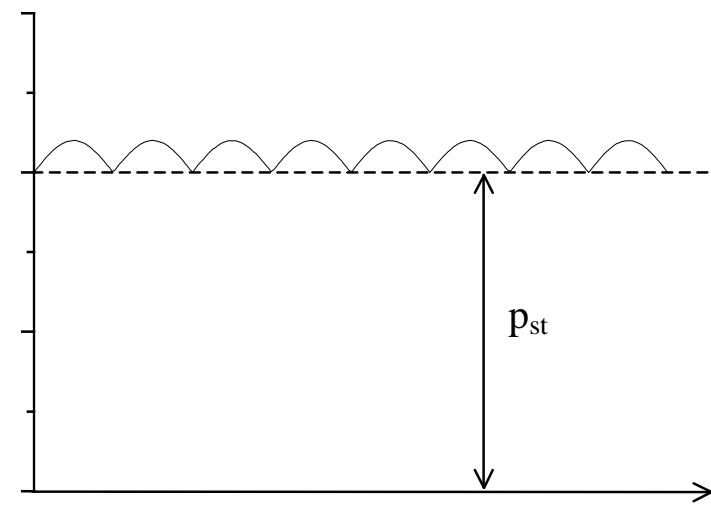

Regime $b$

Abbildung B3: Die zwei Anregungsregime $(a, b)$ der Piezokeramik und die dadurch erzeugten Druckschwingungen in der Zelle.

Die Fourier-Analyse für eine periodische Funktion $\mathrm{y}=|\sin (\mathrm{x})|$ ist:

$|\sin (x)| \approx 2 / \pi-0.424 \cos (2 x)-0.085 \cos (4 x)+\ldots+a_{n} \cos (2 n x), n=1,2,3, \ldots$

dabei ist $\mathrm{a}_{\mathrm{n}}$ nach (b11) bestimmt.

$a_{n}=\frac{4}{\pi} \int_{0}^{\frac{\pi}{2}}|\sin (x)| \cdot \sin (2 n x) \cdot d x$ 
Man sieht sofort, dass das Spektrum der Druckschwingung in der Zelle im Regime b nur geradzahlige Harmonische enthält. Das bedeutet, dass das Signal, das von der Widerstandsmodulation hervorgerufen wird, die Frequenzen $2 f_{1} \pm f_{2}, 4 f_{1} \pm f_{2}$ usw. hat. Diese Frequenzen befinden sich genügend weit weg von der Lock-In-Arbeitsfrequenz und werden zusätzlich noch mit dem Bandpass-Filter abgeschirmt, sodass der Eingang des Lock-InVerstärkers insgesamt nicht überlastet wird. Der Beitrag des von der Widerstandsmodulation hervorgerufenen Signals in der Gerätanzeige ist im Fall (b) gleich Null. Daher können wir die Differenz $U^{\mathrm{a}}$ mess $-\mathrm{U}^{\mathrm{b}}{ }_{\text {mess }}$ als reines Messsignal $U_{\text {mess }}$ betrachten. Da beide Signale (Regime a und b) beide Wechselspannungen mit möglicherweise unterschiedlichen Phasen sind, muss die Differenzbildung unbedingt vektoriell erfolgen.

Im Verlauf der Messung wurde die Anregung alle 3 Sekunden aus dem Regime (a) in das Regime (b) umgeschaltet und umgekehrt. Das ist schnell im Vergleich zur Lock-In-Driftperiode von einigen Minuten, d.h. mit diesem Verfahren haben wir die Drift nahezu ausgeschaltet.

Um das Experiment zwischen beiden Regime umzuschalten, wurde die Keramik plus Wechselspannungsquelle mit Hilfe eines Transformators angekoppelt und gleichzeitig wurde in die Leitung eine vom Computer gesteuerte Gleichspannungsquelle geschaltet (Abbildung B1).

Ist die Amplitude I des Stromes durch die Probe gleich Null, so erwartet man, dass das gesuchte Signal auf der Summenfrequenz $7.5 \mathrm{kHz}$ (die vom Referenzsignal bestimmte Betriebsfrequenz des Lock-In) auch verschwindet. Damit sollte die Gerätanzeige (abgesehen von zufälligen Störungen) auch gleich Null sein. Das Experiment hat aber ergeben, dass das Gerät immer einen ganz bestimmten endlichen Wert anzeigt. d.h., es gibt noch einen parasitäres Signal, das man auch noch abziehen muss. Dabei wird, wie im Fall der zwei verschiedenen Anregungen, die Vektoralgebra verwendet. Dieses Signal ist vermutlich eine Fourier-Komponente des Anregungssystems, die die Filter, die in unserem Aufbau verwendet werden, nicht vollständig unterdrücken können.

Bevor man einen endgültigen Messpunkt bekommt, muss man also folgende Stufen durchlaufen: 1.

- Akkumulation einer großen Anzahl $\left(\sim 10^{3}\right)$ vom Messwerten für $\mathrm{U}^{\mathrm{a}}$ mes. und $\mathrm{U}^{\mathrm{b}}{ }_{\text {mes. }}$ bei einem Strom I durch die Probe, der nicht gleich Null ist.

- Differenzbildung $\mathrm{U}^{\mathrm{a}}{ }_{\text {mes. }}-\mathrm{U}^{\mathrm{b}}{ }_{\text {mes. }}$ für das jeden Paar des Messwerte.

- Mittelung aller Differenzen.

2. Hier wiederholt man alle in 1 beschriebenen Schritte beim Strom $\mathrm{I}=0$ (in beiden Fällen sind Amplitude und Phase des Signals zu speichern).

3. Differenzbildung der nach 1 und 2 erhaltenen Mittelwerte (sehe Abbildung B4). 
4.

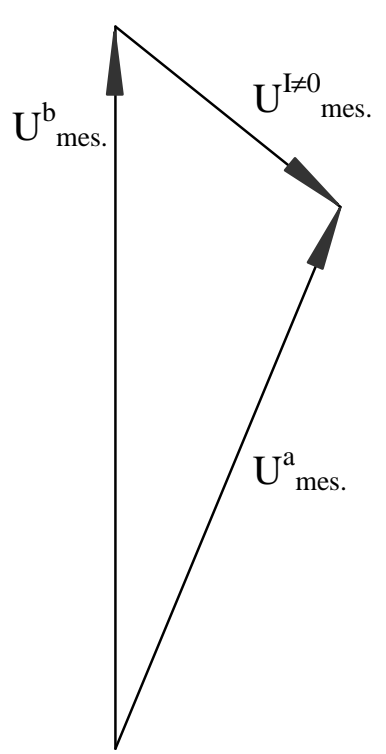

(a)

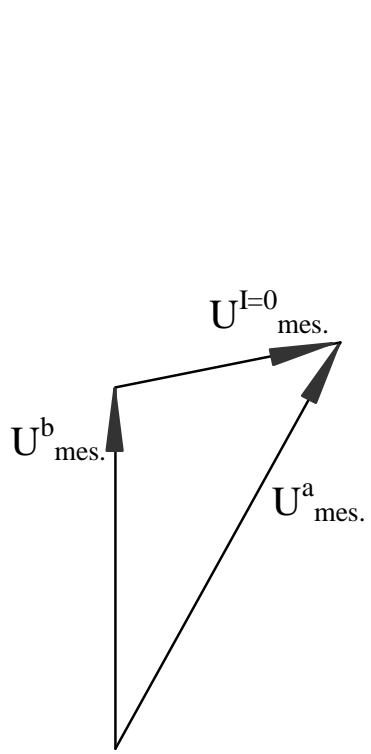

(b)

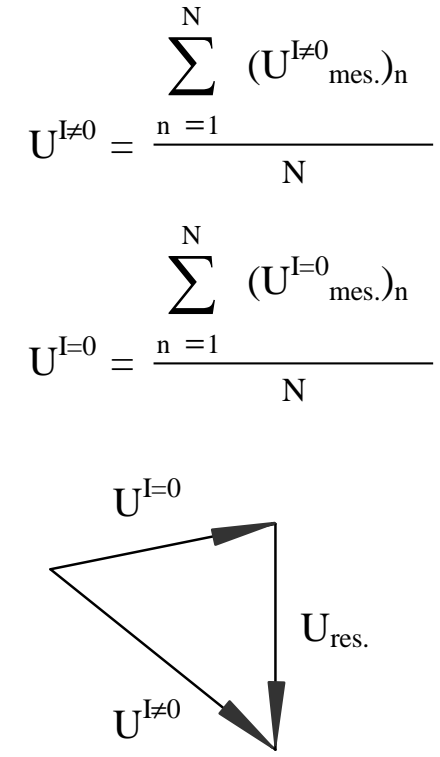

(c)

Abbildung B4: Vektordifferenzbildung: a) für jedes Paar des Messwerte bei einem Strom I durch die Probe; b) für jedes Paar des Messwerte ohne den Strom; c) Differenzbildung für die Mittelwerte von der nach a) und b) erhaltenen Messwerte.

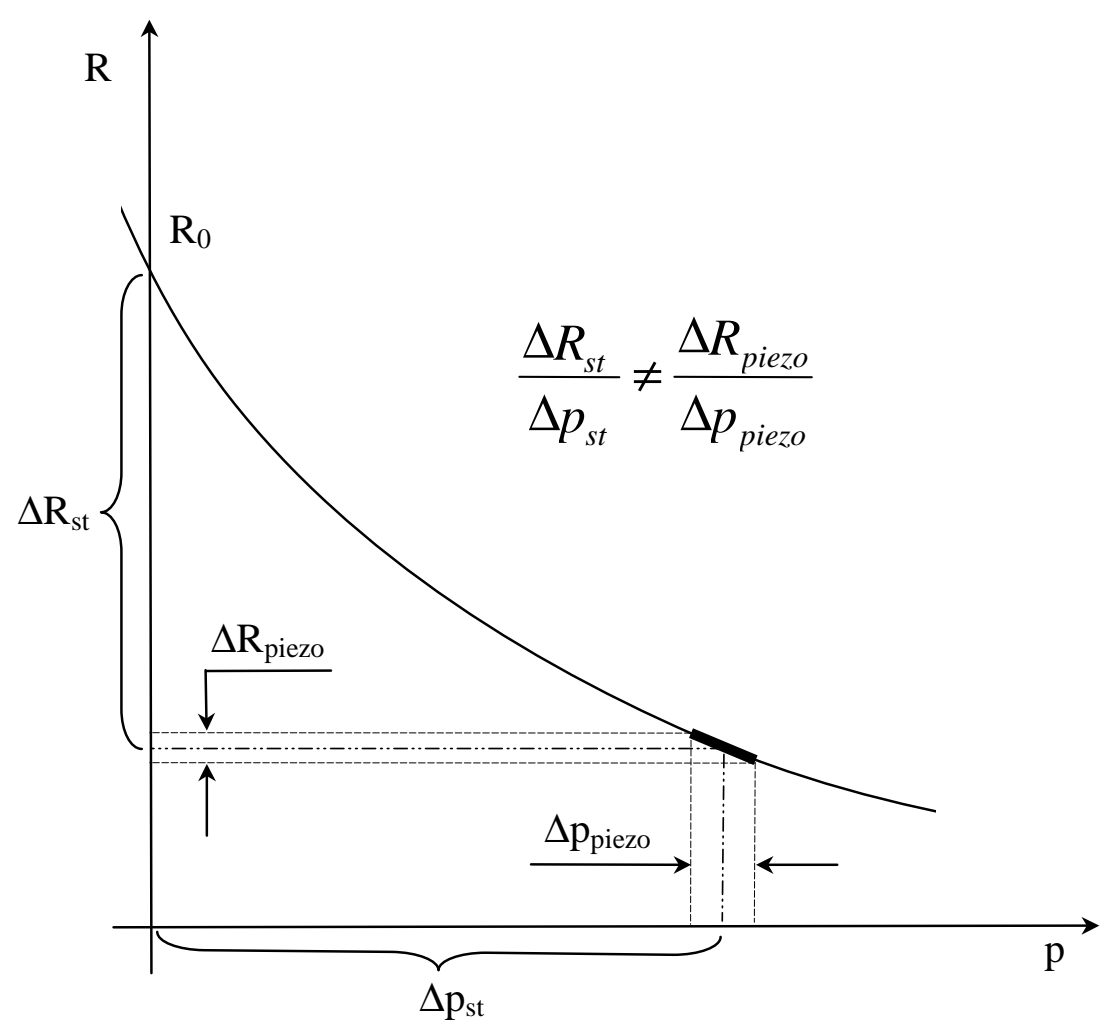

Abbildung B5: Differenzielle Messung des barischen $\gamma$-Koffizienten mit Hilfe der Doppelwechselfeldmethode (schematisch).

Durch Überlagerung eines statischen Druckes $\mathrm{p}_{\mathrm{st}}$ mit einem kleinen Wechseldruck $\mathrm{p}_{\text {piezo }}$ wird mit Hilfe der hier präsentierten Doppelwechselfeldmethode der $\gamma(p)$-Koeffizient gemessen. $\gamma$ ist eine 
differentielle Größe (Abbildung B5). Daher kann man auch ein nichtlineares Verhalten von $\gamma(\mathrm{p})$ bestimmen. Im Vergleich mit der statischen Methode, bei der man aus den R(T)-Kurven unter den verschiedenen statischen Drucken den statischen $\gamma$-Koeffizienten ausrechnen kann, hat diese Methode einen größeren Anwendungsbereich: man kann durch eine Messung in dem sehr kleinen Druckintervall Strukturen im $\gamma(\mathrm{p})$-Verlauf mit sehr viel höherer Präzision detektieren. Diese Methode gibt dem Experimentator ebenfalls eine Möglichkeit, den $\gamma$-Koeffizient für den Druck $\mathrm{p} \rightarrow 0 \mathrm{zu}$ messen, was mit Hilfe der statischen Methode praktisch nicht möglich ist. Bei der Messung $\gamma(\mathrm{p} \rightarrow 0)$ braucht man dann offenbar auch keine komplizierte Hochdruckzelle; ein einfacher hermetischgeschlossener Behälter würde dann ausreichen. Es ist uns insbesondere gelungen, Perkolationseffekte bei Phasengemischen zu detektieren, die in statischen Messungen nicht aufgelöst werden konnten.

\section{Ergebnisse der Doppelwechselfeldmethode}

Der $\gamma$-Koeffizient von Manganin wurde nach dem oben beschriebenen Verfahren gemessen. Allerdings ist der barische Koeffizient des Manganindrahtes in dem in unserem Aufbau zugänglichen Temperaturbereich konstant. Deshalb gibt es keine Besonderheiten im Kurvenverhalten, die uns sagen könnten, dass man mit dem Signal wirklich den differenziellen $\gamma$-Koeffizient misst. Dazu benötigt man eine Probe, bei der $\gamma$ in typischer Weise variiert und von der es klassische Untersuchungen von $\rho(p, T)$ bereits gibt.

In der Arbeit [3] sind beispielweise Widerstandsmessungen an einer keramischen sauerstoffdefizitären $\mathrm{Nd}_{2 / 3} \mathrm{Sr}_{1 / 3} \mathrm{MnO}_{3-\delta}$-Probe unter dem hydrostatischen Druck von 0 bis 15.1 kbar dargestellt. Für Abschätzung des $\delta$ wurden die in Arbeit [5] präsentierten Widerstandsmessungen an den zwei sauerstoffdefizitären Proben zum Vergleich genommen. Die Probe mit $\delta=0$ hat einen Metall-Isolator-Übergang bei $\mathrm{T}_{\mathrm{mi}}=\mathrm{T}_{\mathrm{C}}=250 \mathrm{~K}$. Die zweite Probe mit dem Sauerstoffdefizit $\delta=0.05$ hat den Übergang bei ungefähr $150 \mathrm{~K}$. Die sauerstoffdefizitäre Probe [3] hat aber den Metall-Isolator-Übergang $\mathrm{T}_{\mathrm{mi}}=\mathrm{T}_{\mathrm{C}}$ bei $226 \mathrm{~K}$. Die Temperatur des CuriePunktes hängt sehr stark vom Sauerstoffgehalt ab, so dass man von $\mathrm{T}_{\mathrm{C}}$ auf $\delta$ schließen kann. Aus $\mathrm{T}_{\mathrm{C}}$ ergibt sich das $\delta$ der Probe [3] kleiner als 0.05. Die normierten R(T)-Kurven aus [3] unter den Drucken 0, 3.2, 5.3 und 7.3 kbar sind in Abbildung B6a präsentiert. Daraus ergibt sich der statische $\gamma$-Koeffizient. Das Ergebnis ist in der Abbildung B6(b, c, d) dargestellt. Dabei wurden folgende Intervalle und Formeln benutzt:

$\gamma_{01}=\frac{\Delta R_{01}}{R_{0} \Delta p_{01}}, \gamma_{12}=\frac{\Delta R_{12}}{R_{0} \Delta p_{12}}$ und $\gamma_{23}=\frac{\Delta R_{23}}{R_{0} \Delta p_{23}}$

wobei $\Delta \mathrm{R}_{01}=\mathrm{R}_{0}-\mathrm{R}_{1}, \Delta \mathrm{R}_{12}=\mathrm{R}_{1}-\mathrm{R}_{2}$ und $\Delta \mathrm{R}_{23}=\mathrm{R}_{2}-\mathrm{R}_{3}$ sind $\left(\mathrm{R}_{0}, \mathrm{R}_{1}, \mathrm{R}_{2}\right.$ und $\mathrm{R}_{3}$ sind die den Drucken $\mathrm{p}_{0}=0, \mathrm{p}_{1}=3.2, \mathrm{p}_{2}=5.3$, und $\mathrm{p}_{3}=7.3 \mathrm{kbar}$ entsprechenden Probenwiderstände). $\Delta \mathrm{p}_{01}, \Delta \mathrm{p}_{12}$ und $\Delta \mathrm{p}_{23}$ sind die Differenzen zwischen den Drucken $\mathrm{p}_{0}, \mathrm{p}_{1}, \mathrm{p}_{2}$ und $\mathrm{p}_{3}$. Um eine $\gamma(\mathrm{T})$-Kurve zu bekommen, braucht man also mindestens zwei $\mathrm{R}(\mathrm{T}, \mathrm{p})$-Messungen. 

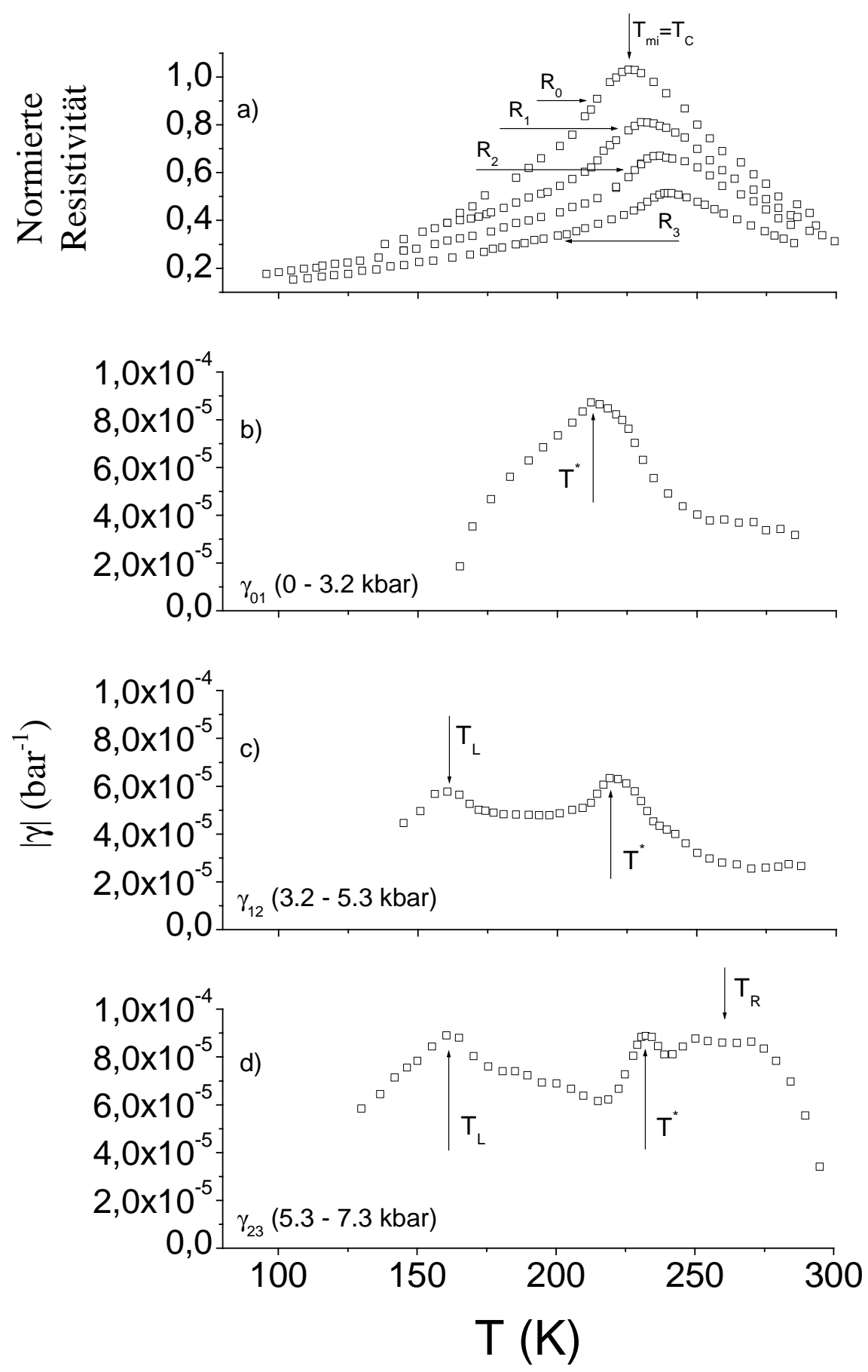

Abbildung B6: a) Normierter Widerstand der Proben $\mathrm{Nd}_{2 / 3} \mathrm{Sr}_{1 / 3} \mathrm{MnO}_{3-\delta}$, gemessen bei 0 kbar, 3.2 kbar, 5.3 und 7.3 kbar (nach Medvedeva[3]); b-d) berechnete $\gamma$-Koeffizienten von $\mathrm{Nd}_{2 / 3} \mathrm{Sr}_{1 / 3} \mathrm{MnO}_{3-\delta}$.

Aus der Abbildung B6a ist zu entnehmen, dass der Curie-Punkt $T_{C}$ bei dieser Probe bei $226 \mathrm{~K}$ liegt, für den Druck 0 kbar. Mit zunehmendem Druck wandert $\mathrm{T}_{\mathrm{C}}$ in Richtung höherer Temperaturen. Dabei wird der absolute Wert des Widerstandes reduziert. Da bei dieser Probe dem größeren Druck ein kleinerer Widerstand entspricht, ist der barische Koeffizient in diesem Fall negativ. In der Abbildung B6 (b, $\mathrm{c}$ und d) wird aber nur der Betrag der $\gamma$-Koeffizienten aufgezeichnet. An allen drei Kurven ist ein lokales Maximum des barischen Koeffizienten $\mathrm{T}^{*} \mathrm{zu}$ finden. Dieses Maximum $\mathrm{T}^{*}$ stimmt mit der entsprechenden Curie-Temperatur nicht überein; es 
liegt immer einige Grad Kelvin tiefer. Mit zunehmendem Druck verschiebt sich $\mathrm{T}^{*}$ jedoch ähnlich wie $\mathrm{T}_{\mathrm{C}}$ nach rechts. Außerdem ist links von $\mathrm{T}^{*}$ bei höheren Drücken $\left(\gamma_{12}: 3.2-5.3\right.$ und $\left.\gamma_{23}: 5.3-7.3 \mathrm{kbar}\right)$ noch ein zweites Maximum $\mathrm{T}_{\mathrm{L}} \mathrm{zu}$ sehen, das bei der $\gamma_{01}(0-3.2 \mathrm{kbar})$ nur als eine Schulter zu beobachten ist. Im rechten Kurventeil (rechts von der $\mathrm{T}^{*}$ ) sieht man bei den höheren Drucken ebenfalls eine Struktur, d.h. mit zunehmendem Druck entwickelt sich ein drittes Maximum. Bei der $\gamma_{01}$ und $\gamma_{12}$-Kurve ist die zusätzliche Schulter kaum noch zu sehen, aber schon bei der nächsten, $\gamma_{23}$-Kurve $\left(5.3-7.3\right.$ kbar) zeigt sich ein deutliches Maximum $\mathrm{T}_{\mathrm{R}}$.

Für unsere Messung mit der Doppelwechselfeldmethode wurde eine $\mathrm{Nd}_{2 / 3} \mathrm{Sr}_{1 / 3} \mathrm{MnO}_{3-\delta^{-}}$ Keramikprobe mit $\delta=0$ ausgesucht. Aus den statischen Messungen weißt man, dass der Widerstand dieser Probe ebenfalls sehr empfindlich auf den Druck reagiert. Weil in unserem Experiment mit sehr kleinen Drucken (vergleichbar mit einem Schalldruck) gearbeitet wird, war dieser hohe $\gamma$-Wert essentiell.

Da die in [3] untersuchte Probe nur einen kleinen Sauerstoffdefizit hat, kann sie doch noch als Vergleichsprobe benutzt werden. Dazu muss man allerdings reduzierte Temperaturen $\mathrm{T}_{\mathrm{C}}(\mathrm{p}) / \mathrm{T}_{\mathrm{C}}(0)$ benutzen (siehe unten). Dann erwarten wir von dem nach der Doppelfeldmethode gemessenen und den klassischen $\gamma$-Koeffizienten ein ähnliches Verhalten.

Der mit der Doppelwechselfeldmethode gemessene barische Koeffizient der $\mathrm{Nd}_{2 / 3} \mathrm{Sr}_{1 / 3} \mathrm{MnO}_{3^{-}}$ Probe beim zusätzlichen statischen Offset-Druck $\mathrm{p}_{\mathrm{st}}=1 \mathrm{kbar}$ wird in Abbildung B7 zusammen mit dem aus der statischen Messung $\left(\gamma_{01}\left(\mathrm{~T} / \mathrm{T}_{\mathrm{C}}\right)\right.$-Kurve, siehe obere Grafik) verglichen. $\gamma_{01}$ beschreibt die Widerstandsänderung zwischen 0 und 3.2 kbar und entspricht daher am besten dem Offset-Druck von 1 kbar. Um die Messungen bezüglich T vergleichen zu können, wurde die Temperaturskala auf die entsprechenden Curie-Temperaturen normiert. Nur so $(\gamma$-Werte gegen $\mathrm{T} / \mathrm{T}_{\mathrm{C}}$ ) kann man die charakteristischen Merkmale dieser Kurven vergleichend diskutieren.

In der Abbildung ist zu sehen, dass die nach der Doppelwechselfeldmethode gemessene Kurve ähnlich wie die statische Messung ein Maximum links von Curie-Punkt hat. Der Absolutwert des Maximums beträgt $\approx 10^{-4} \mathrm{bar}^{-1}$. Dieser Wert stimmt gut mit den aus der Arbeit [3] genommenen Daten überein. Also hat das mit Hilfe der neuen Methode gewonnene $\gamma$ ein ähnliches Verhalten wie das statisch gemessene $\gamma$, abgesehen von dem ausgefüllten Punkt (Abbildung B7b, ausgezeichnet mit einem Kreis), der reel ist und unten weiter diskutiert wird. 


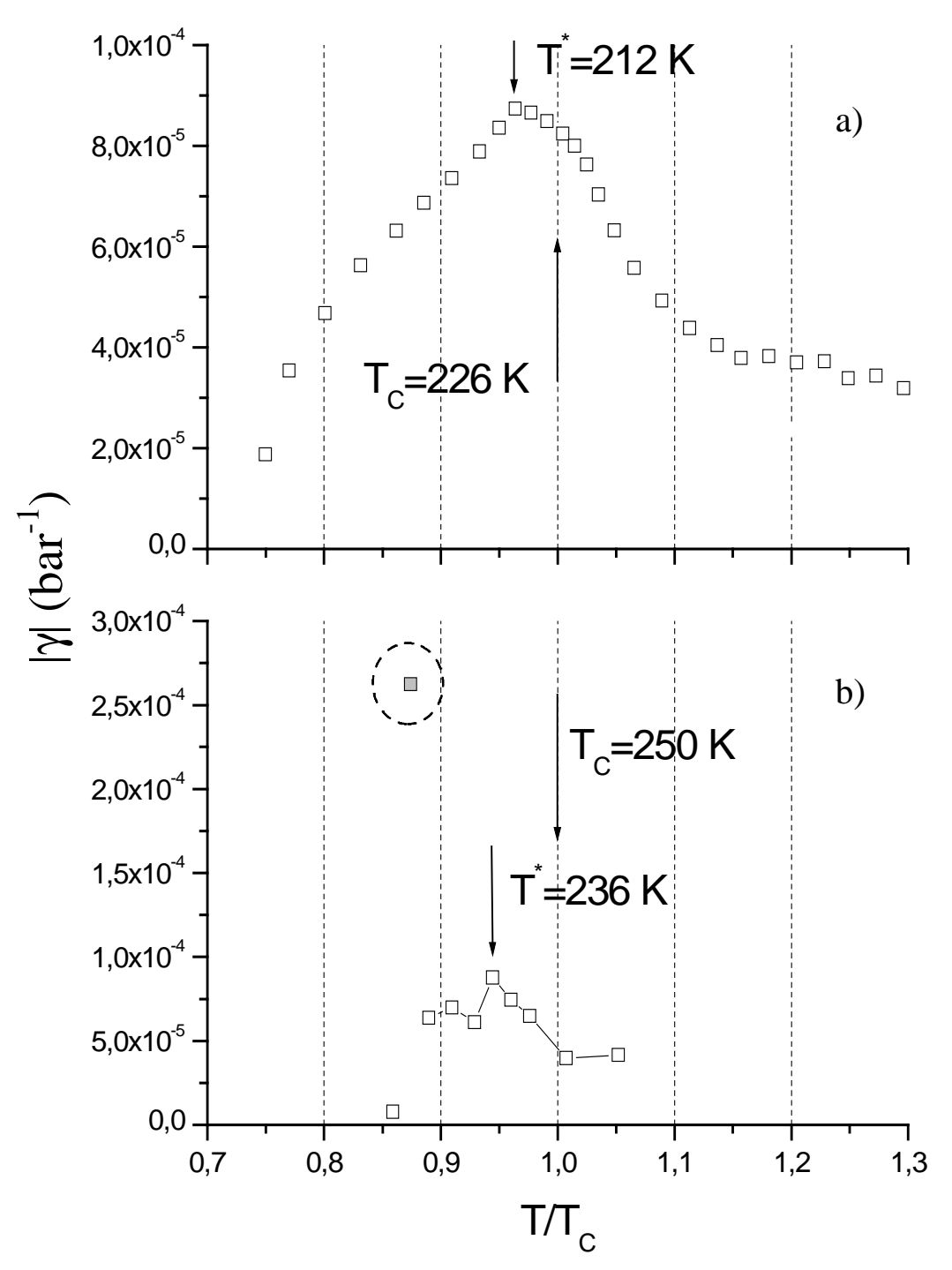

Abbildung B7: a) statisch gemessener $\gamma_{01}$-Koeffizient, ausgerechnet aus den $R(T)$-Kurven zwischen 0 und 3.2 kbar. b) $\gamma$-Koeffizient, gemessen mit der Doppelwechselfeldmethode beim Offset-Druck 0 kbar.

Mit der neu entwickelten Mehode wurde der barische Koeffizient auch unter den Offset-Drucken 0 und 2 kbar gemessen. Die entsprechende Kurven sind zusammen mit der Messung unter dem Offset-Druck 1 kbar in der Abbildung B8 ( $-0, b-1, c-2$ kbar) aufgetragen. Alle drei Messungen haben ein in der Nähe von $T_{C}$ liegendes Maximum im Kurvenverlauf, das man dem Temperaturpunkt $\mathrm{T}^{*}$ aus den statischen Messungen zuordnen kann. Bei den höheren Drucken (1 und 2 kbar) findet man, dass $\mathrm{T}^{*}$ wie bei den statischen Messungen mit zunehmendem Druck zunimmt. Bei der 1 und 2 kbar-Messung findet man rechts von der $\mathrm{T}^{*}$ einen Bereich, in dem der $\gamma$-Koeffizient eine wieder aufsteigende Tendenz zeigt. Man kann daher erwarten, bei höheren Temperaturen wieder ein Maximum $\mathrm{T}_{\mathrm{R}} \mathrm{zu}$ finden. Dieses Maximum entspricht dann dem Maximum $\mathrm{T}_{\mathrm{R}}$ aus den statischen Messungen. 

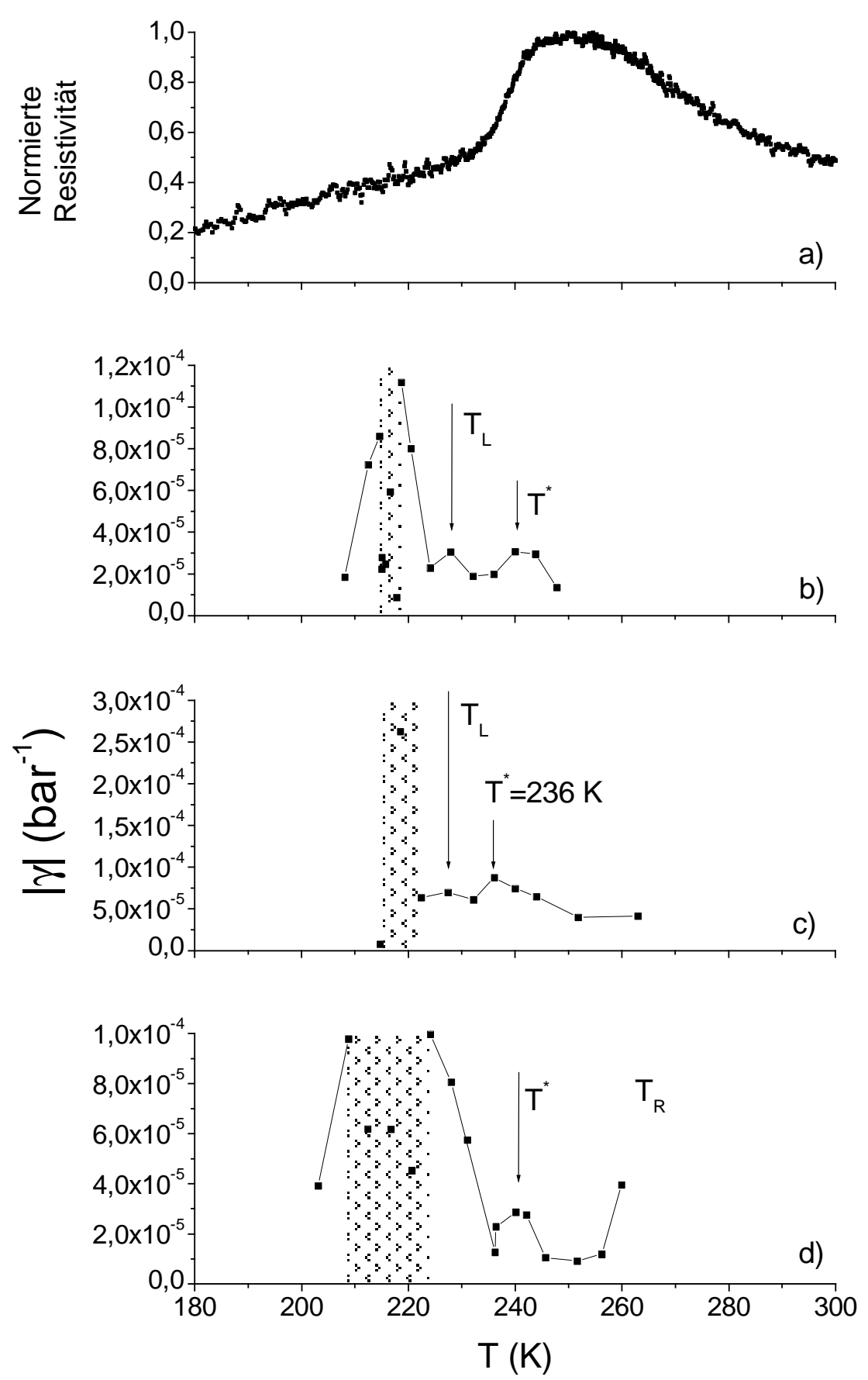

Abbildung B8: Die bei 0 kbar gemessene normierte Resistivität der $\mathrm{Nd}_{2 / 3} \mathrm{Sr}_{1 / 3} \mathrm{MnO}_{3}$-Probe (a) und die mit Hilfe der Doppelwechselfeldmethode gemessene $\gamma$-Koeffizienten unter dem verschiedenen Offset-Drucken ( $b-0$ kbar, $c-1$ kbar, $d-2$ kbar).

Generell ist die Struktur der mit der Doppelwechselfeldmethode gewonnenen $\gamma(\mathrm{T})$-Kurven viel schärfer und deutlicher als bei den statischen Messungen. So etwas erwartet man aber auch, wenn man eine differenzielle Methode einsetzt.

Im linken Teil der $\gamma(T)-K u r v e n$ wurde überraschenderweise ein Bereich entdeckt, bei dem bei festem T stets ein stabiler Messwert erhalten wurde, aber niemals derselbe Wert. Der Bereich ist in der Abbildung B8 als schraffierte Zone ausgezeichnet. Solche Anomalien werden bei den 
statischen Messungen nicht gefunden. Das Auftreten dieses instabilen Bereiches bezieht sich daher vermutlich auf spezielle Probeneigenschaften und auch auf die differenzielle Natur der verwendeten Methode. Der instabile Bereich liegt vor dem linken Maximum, das man dem Maximum $\mathrm{T}_{\mathrm{L}}$ aus den statischen Messungen zuordnen kann. Offenbar sieht man unterhalb von $\mathrm{T}_{\mathrm{C}}$ ein Phasenseparationsphänomen. Da dieser Bereich bei tieferen Temperaturen liegt, kann es dabei nur um die Perkolation von isolierenden pm-Clusters handeln. Eine solche Perkolation kann aber nicht zu dem so großen Effekt führen, da es sich um die Ausbildung von der linearen Ketten innerhalb des Volumens handelt. Eine metallische Matrix bleibt trotzdem immer kurzgeschlossen. Zur Erklärung dieses $\gamma$-Bereiches wird die Existenz der Korngrenzen herangezogen. Da die den Korngrenzen naheliegenden Bereiche immer einen Sauerstoffdefizit $\delta$ haben, können sie bei einer Temperatur bereits paramagnetisch sein, bei der die Volumenbereiche noch metallisch sind. In diesem Fall sieht die Gesamtprobe insgesamt isolierend aus. Wenn die Probe die Temperatur hat, die der Perkolationsschwelle im Nahbereich entspricht, kann der Gesamtwiderstand schon mit geringsten Druck stark verändert werden. Dabei reicht der Übergang eines kleinen paramagnetischen Clusters in dem Korngrenzenbereich in den metallischen Zustand aus. Da wir hier eine differenzielle Methode verwenden, führt diese auf sprungartige im $\gamma$-Koeffizienten. 


\section{Literaturverzeichnis}

[1] M. Sasaki, H. Negishi, M. Inoue.

Pulsed laser-induced transient thermoelektric effects in silicon crystals.

J. Appl. Phys. 59 (1986) 796

[2] Jörg Liebe

Untersuchungen stabiler und lichtinduzierter Defekte in a-Si:H durch die Photoleitungsfrequenzmischung und zeitaufgelöste thermoelektrische Effekte.

Dissertation zur Erlangung des Doktorgrades an der Universität Göttingen, Göttingen 1998.

[3] I. V. Medvedeva, Yu.S. Bersenev, K. Baärner, L. Haupt, R. Mandal, A. Poddar

The influence of hydrostatic pressure on the temperature dependence of the resistivity in $\mathrm{Nd}_{2 / 3} \mathrm{Sr}_{1 / 3} \mathrm{MnO}_{3-\delta}$ Physica B 229 (1997) 194-198

[4] C.P. Yang, V. Morchshakov, Y.L. Huang, I.O. Troyanchuk, K. Bärner.

Boundary resistance anomalies in $\mathrm{Nd}_{0.7} \mathrm{Sr}_{0.3} \mathrm{MnO}_{3-\delta}$.

Physica B 337 (2003) 287-291

[5] V. Morchshakov, K.Bärner, A.Kattwinkel, A.M. Ahmed, I. El-Kassab, G.H. Rao, I.V. Medvedeva.

Poster: Heat conductivity and oxygen non-stoichiometry related phonon modes.

The European Conference PHYSICS OF MAGNETISM'02; 1-5 July, 2002 Poznan.

[6] W. Schnelle, A. Poddar, P. Murugaraj, E. Gmelin, R. K. Kremer, K. Sasaki, J. Maier.

The effect of annealing conditions on the physical properties of $\mathrm{Nd}_{0.67} \mathrm{Sr}_{0.33} \mathrm{MnO}_{3-\delta}$

J. Phys.: Condens. Matter 12 (2000) 4401

[7] K. Bärner, B. Raveau and I. O. Troyanchuk.

Some elements of oxygen non-stoichiometry in manganites.

Recent Res. Devel. Mat. Sci. Engg., 2(2003): 185-216 ISBN: 81-7895-091-X

[8] I. El-Kassab, A. M. Ahmed, P. Mandal, K. Bärner, A. Kattwinkel, U. Sondermann

Heat conductivity of $\mathrm{La}_{1-\mathrm{x}} \mathrm{Sr}_{\mathrm{x}} \mathrm{MnO}_{3}$ surface layers.

Physica B, 305 (2001) 233-241

[9] A. Asamitsu, Y. Tomioka, H. Kuwahara \& Y. Tokura

Current switching of resistive states in magnetoresistive manganites.

Nature 388, 50 - 52 (03 July 1997)

[10] C. P. Yang, V. Morchshakov, I. O. Troyanchuk, G. H. Rao, K. Bärner

Evidence for an electrical field induced order-order transiotion in $\mathrm{Nd}_{0.7} \mathrm{Sr}_{0.3} \mathrm{MnO}_{\mathrm{z}}$ Journal of Alloys and Compounds 383 (1-2), pp. 45-48

[11] A. Poddar, P. Muruguraj, R. Fischer, E. Gmelin, K. Bärner, L. Haupt, P. Mandal and G. H. Rao Hall measurements on $\mathrm{La}_{2 / 3} \mathrm{Ca}_{1 / 3} \mathrm{MnO}_{3-\delta}$ and $\mathrm{Nd}_{2 / 3} \mathrm{Sr}_{1 / 3} \mathrm{MnO}_{3-\delta}-$ a comparative study Physica B: Condensed Matter, Volume 254, Issues 1-2, 2 November 1998, Pages 21-27

[12] H. L. Ju, J. Gopalakrishnan, J. L. Peng, G. C. Qi Li, T. Xiong, R. L. Venkatesan, Greene Dependence of giant magnetoresistance on oxygen stoichiometry and magnetization in polycrystalline $\mathrm{La}_{0.67} \mathrm{Ba}_{0.33} \mathrm{MnO}_{\mathrm{z}}$

Phys. Rev. B 51 (1995) 6143

[13] L. Haupt, R. V. Helmolt, U. Sondermann, K. Bärner, Y. Tang, E.R. Giessinger, E. Ladizinski, R. Braunstein

Phys. Lett. A 165 (1992 473)

[14] T. Gron, K. Bärner, Ch. Kleeberg, I. Okonska-Kozlowska

The thermoelectric power of ferromagnetically ordered $\mathrm{Cu}_{1-\mathrm{x}} \mathrm{Ga}_{\mathrm{x}} \mathrm{Cr}_{2} \mathrm{Se}_{4}$ spinels

Physica B 225 (1996) 191 
[15] K. Bärner, P. Mandal, R. V. Helmolt

Positive and Negative Magnetoresistance in Mixed Superexchange-Double Exchange Systems Phys. Stat. Sol. B 223 (2001) 811

[16] V. Morchshakov, C. P. Yang, I. O. Troyanchuk and K. Bärner. Electroresistive effect in slightly oxygen deficient $\mathrm{Nd}_{0.7} \mathrm{Sr}_{0.3} \mathrm{MnO}_{3-\delta}$ ceramic.

Journal of Alloys and Compounds, In Press, Corrected Proof, Available online 26 April 2005

[17] Nagat El-Sebaey Farag Hamad,

Dissertation zur Erlangung des Doktorgrades an der Universität Göttingen, Göttingen 2003.

[18] J. Liebe, H. Kang, L. Haupt, P. Mandal, I. V. Medvedeva, K. Bärner, A. Poddar,

E. Gmelin

Heat diffusivity of $\mathrm{Nd}_{1-\mathrm{x}} \mathrm{Sr}_{\mathrm{x}} \mathrm{MnO}_{3-\delta}$ compounds.

Physica B 239 (1997) 322-327

[19] Vincent H. Crespi, Li Lu, Y. X. Jia, K. Khazeni, A. Zettl and Marvin L. Cohen

Thermopower of single-crystal $\mathrm{Nd}_{1-\mathrm{x}}(\mathrm{Sr}, \mathrm{Pb})_{\mathrm{x}} \mathrm{MnO}_{3-\delta}$.

Physical Review B, (1996) 53 (21) 14303-14308

[20] M. Jaime, M. B. Salamon and K. Pettit

Magnetothermopower in $\mathrm{La}_{0.67} \mathrm{Ca}_{0.33} \mathrm{MnO}_{3}$ thin films

Appl. Phys. Lett. 68 (11) 1676-1578

[21] W. Prellier, B. Mercey, Ph. Lecoeur, J. F. Hamet, B. Raveau

Tailored superlattices containing distinct oxide and oxycarbonate blocks grown by pulsed laser deposition. Appl. Phys. Lett. 71 (6) 782 (1997)

[22] K. Bärner, P. Mandal and R. v. Helmolt

Positive and negative magnetoresistance in mixed superexchange - double exchange Systems.

Phys. Stat. Sol. (b) 223, 811 (2001)

[23] Ch. Kleeberg, A. Vetcher, H. Dunkel, J. W. Schünemann and K. Bärner

Field- and anisotropy-induced states in $\mathrm{MnAs}_{1-\mathrm{x}} \mathrm{P}_{\mathrm{x}}$-single crystals

J. Alloys Compd. 262502 (1997)

[24] P. G. deGennes

Phys. Rev. 118, 141 (1960)

[25] A. Maignan, Ch. Simon, V. Caignaert and B. Raveau Physica B 99, 305 (1996)

[26] G. H. Rao, J. R. Sun, A. Kattwinkel, L. Haupt, K. Bärner, E. Schmitt and E. Gmelin Magnetic, electric and thermal properties of $\mathrm{La}_{0.7} \mathrm{Ca}_{0.3} \mathrm{Mn}_{1-\mathrm{x}} \mathrm{Fe}_{\mathrm{x}} \mathrm{O}_{3}$ compounds

Physika B 269, 379 (1999)

[27] C. Zener

Phys. Rev. 81, 440 (1951)

[28] Tokura Y.

Colossal magnetoresistive manganites

JMMM. 1999. v.200. p.1-23.

[29] Rao C.N.R.

Giant magnetoresistance, charge ordering and other novel properties of perovskite manganites J.Phys. Chem.Solids.1998. v.59. N4. p.487-502.

[30] Ramirez A.P.

Colossal magnetoresistance.

J.Phys.:Condenc.Matter 1997. v.9. p.8171-8199. 


\section{Literaturverzeichnis}

[31] Salamon M.B.

The physics of manganites: Structure and transport //Rev.Mod.Phys.2001. v.73. p.583-626.

[32] Coey J.M.D., Viret M., Ranno L., Ounadjela K.

Electron localization in mixed-valence manganites

Phys.Rev. B. 1995. v.75. N21. p.3910 - 3913.

[33] Troyanchuk I,O., Efimov D.A., Szymczak H., Szymczak R., Krzymanska B.

Effects of oxygen deficit, $\mathrm{Sr}$, Ti and $\mathrm{Cr}$ substitution on the antiferro-ferromagnet transition in

$\mathrm{Nd}_{0.6} \mathrm{Ca}_{0.4} \mathrm{MnO}_{3}$.

JMMM, 1999, v.202, p.95-99.

[34] Bärner K., Haupt L., v.Helmolt R.

Comment on electrical transport in oxide based double exchange ferromagnets. Phys.sat.sol.(b) 1995.

V.187.p. K61-K65.

[35] Anderson P.W., Hasegawa H.

Consideration on doulble exchange.

Phys.Rev. 1955. v.100. p.675-681

[36] Radaelli P.G., Iannone G., Marezio M., Hwang H. Y., Cheong S. -W., Jorgensen J. D. and Argyriou D. N. Structural effects on magnetic and transport properties of perovskite $\mathrm{A}_{1-\mathrm{x}} \mathrm{A}_{\mathrm{x}} \mathrm{MnO}_{3}(\mathrm{x}=0.25,0.30)$.

Phys. Rev. B. 1997. v 56. N13. p. 8265-8276.

[37] De Teresa J.M., Ibarra M.R., Blasco J., Garcieda J., Marquina C., Argabel P.A, Arnold Z., Kamenev K., Ritter C., Von Helmolt R

Spontaneous behavior and magnetic field and pressure effects on $\mathrm{La}_{2 / 3} \mathrm{Ca}_{1 / 3} \mathrm{MnO}_{3}$ perovskite.

Phys.Rev.B. 1996. v. 54. N2. p. 1187-1193.

[38] Lee S., Hwang H.Y., Shraiman Boris I., Ratcliff II W.D., Cheong S.-W.

Intergrain magnetoresistance via second-order tunelling in perovskite manganites. Phys.Rev.Lett. 1999. v.82. N22. p.4508-4511.

[39] Maignan A., Martin C., Hervieu M., Raveau B.

Intragrain and intergrain magnetiresistance in $\mathrm{Mn}, \mathrm{Fe} / \mathrm{Mo}$ and $\mathrm{Co}$ simple, double and oxygen deficient perovskite oxides.

JMMM. 2000. v.211. p. 173-179.

[40] Kajimoto R., Yoshizawa H., Kawano H., Kuwahara H., Tokura Y.

Hole concentration induced transformation of the magnetic and orbital structures in $\mathrm{Nd}_{1-\mathrm{x}} \mathrm{Sr}_{\mathrm{x}} \mathrm{MnO}_{3}$.

Phys.Rev.B. 1999-1. v.50. N13. p.9506-9518.

[41] Maezono R., Ishihara S., Nagaosa N.

Phase diagram of manganese oxides.

Phys.Rev.B. 1998-1. v.58. N17. p.11583-11597.

[42] Tokura Y., Tomioka Y., Kuwahara H., Asamitsu A., Moritomo Y., Kasai M.

Origin of colossal magnetoresistance in perovskite-type manganese oxides.

J.Appl.Phys. 1996. v.79. p. 5288-5290.

[43] Caignaert V., Maignan A., Raveau B.

Up to $50000 \%$ resistance variation in magnetoresistive polycrystalline perovskites $\mathrm{Ln}_{2 / 3} \mathrm{Sr}_{1 / 3} \mathrm{MnO}_{3}(\mathrm{Ln}=$ $\mathrm{Nd}, \mathrm{Sm})$.

Solid state Comm., 1995.v.95. N6. p.357-359.

[44] Y. Moritomo and A. Asamitsu, Y. Tokura

Pressure effect on the double-exchange ferromagnet $\mathrm{La}_{1-\mathrm{x}} \mathrm{Sr}_{x} \mathrm{MnO}_{3}(0.15 \leq \mathrm{x} \leq 0.5)$

Phys. Rev. B 51, 16491 (1995) 
[45] J. J. Neumeier, M. F. Hundley, J. D. Thompson, and R. H. Heffner Substantial pressure effects on the electrical resistivity and ferromagnetic transition temperature of $\mathrm{La}_{1-\mathrm{x}} \mathrm{Ca}_{\mathrm{x}} \mathrm{MnO}_{3}$

Phys. Rev. B 52, 7006 (1995)

[46] A. Urushibara, Y. Moritomo, T. Arima, A. Asamitsu, G. Kido, Y. Tokura Insulator-metal transition and giant magnetoresistance in $\mathrm{La}_{1-\mathrm{x}} \mathrm{Sr}_{\mathrm{x}} \mathrm{MnO}_{3}$ Phys. Rev. B 51 (1995) 14103

[47] C. Searle, S. Wang,

Can. J. Phys. 47 (1969) 2703

[48] J. B. Goodenough

Theory of the Role of Covalence in the Perovskite-Type Manganites [La, M(II)] $\mathrm{MnO}_{3}$

Phys. Rev. 100 (1955) 564

[49] S. K. Hasanain, M. Nadeem, Wiqar Hussain Shah, M. J. Akhtar and M. M. Hasan Effects of iron doping on the transport and magnetic behaviour in $\mathrm{La}_{0.65} \mathrm{Ca}_{0.35} \mathrm{Mn}_{1-\mathrm{y}} \mathrm{Fe}_{\mathrm{y}} \mathrm{O}_{3}$.

J. Phys.: Condens. Matter 12 (2000) 9007-9017

[50] E. Dagotto, T. Hotta and A. Moreo

Colossal magnetoresistant materials: the key role of phase separation

Physics Reports 344 (2001) 1-153

[51] S. Srivastava, N. K. Pandey, P. Padhan, R. C. Budhani

Current switching effects induced by electric and magnetic fields in Sr-substituted $\mathrm{Pr}_{0.7} \mathrm{Ca}_{0.3} \mathrm{MnO}_{3}$ films Phys. Rev. B 62 (2000) 13868

[52] J. Stankiewicz, J. Sese, J. Garcia, J. Blasco, C. Rillo

Magnetic behavior of $\operatorname{Pr}_{1-\mathrm{x}} \mathrm{Ca}_{\mathrm{x}} \mathrm{MnO}_{3}$ in the electric-field-driven insulator-metal transition

Phys. Rev. B61 (2000) 11236

[53] M. E. Lines and A. M. Glass

Principles and applications of ferroelectrics and related materials.

Clarendon Press, oxford 1977, p.8, 69

[54] Editor: Klaus Bärner

New trends in the charakterisation of CMR-manganites and related materials

Research Signpost, 2005, ISBN: 81-308-0043-8

[55] J.B. Goodenough

Magnetism and chemical bond.

Interscience Publishers, New York (1963)

[56] Thomas Becker

Untersuchung der elektrischen Phasenseparation in dünnen Manganatschichten mit

Rastersondenspektroskopie.

Dissertation zur Erlangung des Doktortitels, Georg-August-Universität Göttingen, Mathematischnaturwissenschaftliche Fakultäten, 2004-06-08

[57] P. Schiffer, A. P. Ramirez, W. Bao, and S-W. Cheong

Low Temperature Magnetoresistance and the Magnetic Phase Diagram of $\mathrm{La}_{1-\mathrm{x}} \mathrm{Ca}_{\mathrm{x}} \mathrm{MnO}_{3}$

Phys. Rev. Lett. 75, 3336 (1995)

[58] Y. Tokura und Y. Tomioka,

J. Magn. Magn. Mater. 200, 1 (1999).

[59] M. Kagan, D. Khomskii, M. Mostovoy

Eur. Phys. J. B12, 217 (1999)

[60] S. von Molnar, J. M. D. Coey,

Current Opinion in Solid State and Materials Science 3, 171 (1998). 


\section{Literaturverzeichnis}

[61] A. Moreo, S. Yunoki, E. Dagotto Science 283, 2034 (1999).

[62] H. A. Jahn und E. Teller

Proc. Roy. Soc. (London) 161, 220 (1937).

[63] R. Englman

The Jahn-Teller effect in molecules and crystals.

Wiley Interscience, New York (1972).

[64] E. L. Nagaev

Phys. Stat. Sol. (b) 1869 (1994)

[65] E. L. Nagaev

Physica C 222324 (1994)

[66] E. L. Nagaev

Physica B 9859 (1995)

[67] R. M. Kusrers, J. Singleton, D. A. Keen, R. McGreevy and W. Hayes Physica B, 155, 362

[68] G. C. Xiong, S. Bhagat, Q. Li, M. Dominguez, H. Ju, R. Greene and T. Venkatesan Solid St. Commun., 97, 599. (1996)

[69] A. Maignan, C. Simon, V. Caignaert and B. Raveau J. Magn. magn. Mater., 152, L5 (1996).

[70] G. J. Snyder, R. Hiskes, S. DiCarolis, M. R. Beasley and T. H. Geballe Intrinsic electrical transport and magnetic properties of $\mathrm{La}_{0.67} \mathrm{Ca}_{0.33} \mathrm{MnO}_{3}$ and $\mathrm{La}_{0.67} \mathrm{Sr}_{0.33} \mathrm{MnO}_{3} \mathrm{MOCVD}$ thin films and bulk material Phys. Rev. B, 53, 14434 (1996).

[71] S. Tamura

Phys. Lett. A 78401 (1980)

[72] E. L. Nagev

Colossal-magnetiresistance matirial: manganites and conventional ferromagnetic semiconductors.

Physics Reports 346 (2001) 387-531

[73] F. Millange, V. Caignaert, G. Mather, E. Suard and B. Raveau

Low temperature orthorombic to monoclinic transiotion due to size effect in $\mathrm{Nd}_{0.7} \mathrm{Ca}_{0.3-\mathrm{x}} \mathrm{Sr}_{\mathrm{x}} \mathrm{MnO}_{3}$ :

Evidence for a new type of charge ordering.

Journal of Solid Satate Chemistry 127, 131-135 (1996)

[74] N.-C. Yeh, R. Vasquez, D. Beam, C.-C. Fu, H. Huynh and G. Beach

J. Phys.: condens. Matter, 9, 3713 (1996).

[75] N.-C. Yeh, C. C. Fu, J. Y. T. Wei, R. P. Vasquez et al., J. appl. Phys., 81, 97 (1997).

[76] G. Jakob, W. Westerburg, F. Martin and H. Adrian Small-polaron transport in $\mathrm{La}_{0.67} \mathrm{Ca}_{0.33} \mathrm{MnO}_{3}$ thin films Phys. Rev. B, 58, 14966 (1988).

[77] Y. X. Jia, L. Lu, K. Khazeni, V. H. Crespi, A. Zettl and M. L. Cohen

Magnetotransport properties of $\mathrm{La}_{0.6} \mathrm{~Pb}_{0.4} \mathrm{MnO}_{3-\delta}$ and $\mathrm{Nd}_{0.6}\left(\mathrm{Sr}_{0.7} \mathrm{~Pb}_{0.3}\right)_{0.4} \mathrm{MnO}_{3-\delta}$ single crystals Phys. Rev. B, 52, 9147 (1995). 
[78] M. Viret, L. Ranno and J. M. D. Coey

Magnetic localization in mixed-valence manganites

Phys. Rev. B, 55, 8067 (1997).

[79] R. von Helmholt, L. Haupt, K. Bärner and U. Sondermann

Solid St.Commun., 82, 693 (1992).

[80] J. M. DeTeresa, M. R. Ibarra, J. Blasco, J. Garcia, C. Marquina, P. Algarabel, Z. Arnold, K. Kamenez, C. Ritter and R. von Helmolt

Spontaneous behavior and magnetic field and pressure effects on $\mathrm{La}_{2 / 3} \mathrm{Ca}_{1 / 3} \mathrm{MnO}_{3}$ perovskite

Phys. Rev. B, 54, 1187 (1996).

[81] J. M. D. Coey, M. Viret and S. von Molnar

Mixed-valence manganites

Advances in Physics, Vol. 48, No. 2, 167-293 (1999)

[82] J. B. Goodenough

Magnetism and the chemical bond.

New York: Wiley-Interscience

[83] E. O. Wollan and W. C. Koehler

Phys. Rev. 100, 545 (1955)

[84] J. B. A. A. Elemans, K. R. van Laar, K. R. van der Veen end B. O. Loopstra

J. solid-st. Chem. 3, 238 (1971)

[85] M. Pattabiram, P. Marugaraj, G. Rangarajan, C. Dimitropoulos, J.-P. Ansermet, G. Papavassiliou,

G. Balakrishnan, D. McK. Paul, M.R. Lees

${ }^{55} \mathrm{Mn}$ NMR investigation of $\mathrm{Nd}_{1-\mathrm{x}} \mathrm{Sr}_{\mathrm{x}} \mathrm{MnO}_{3}(0.1<\sim \mathrm{x}<\sim 0.5)$

Phys. Rev. B 66, 224415 (2002)

[86] T. Y. Koo, V. Kiryukhin, P. A. Sharma, J. P. Hill, S.-W. Cheong

Magnetic-field-induced collapse of charge-ordered nanoclusters and the colossal magnetoresistance effect in $\mathrm{Nd}_{0.7} \mathrm{Sr}_{0.3} \mathrm{MnO}_{3}$

Phys. Rev. B 64, 220405 (2001)

[87] M. Pattabiram, P. Marugaraj, G. Rangarajan, V. Prasad, S. V. Subramanyam, V. S. Sastry, S.-M. Koo, K. V. Rao

Pramana-J. Phys. 55, 455 (2000)

[88] M. Faeth, S. Freisem, A. A. Menovsky, Y. Tomioka, J. Aarts, J. A. Mydosh

Science 285 (1999) 1540,

[89] D. McK. Paul, K. Kamenev, G. Balakrishnan, A. J. Campbell, M. R. Lees, G. J. McIntyre

Philos. Trans. R. Soc. London, Ser. A 356 (1998) 1543

[90] I. Medvedeva, K. Bärner, V. Morchshakov, C. Martin, B. Raveau

Volume Effect on Magnetic Transitions in $\mathrm{Pr}_{1-\mathrm{X}} \mathrm{Sr}_{\mathrm{x}} \mathrm{MnO}_{3}$

Poster, IEEE International Magnetics Conference 2003, Boston

[91] K. Bärner, I. V. Medvedeva, E. A. Zavadskii

Pressure dependence of the MI-transition temperature under competing double exchangesuperexchange interactions close to $\mathrm{CDO}$ afm insulating states.

Physica B 355 (2005) 134-139

[92] I. V. Medvedeva, Y. S. Bersenev, K. Bärner, L. Haupt, P. Mandal, A. Poddar

Physica B 229 (1997) 194 


\section{Literaturverzeichnis}

[93] C. N. R. Rao, B. Raveau (Eds.)

Colossal Magnetoresistance, Charge Ordering and Related Properties of Manganese Oxides.

World Scientific, Singapore, pp. 98, 108; ISNB 981-02-3276-4

[94] I. V. Medvedeva, K. Bärner, G. H. Rao, N. Hamad, Yu. S. Bersenev, J. R. Sun

Physica B 292 (2000) 250

[95] Y. Moritomo, A. Asamitsu, Y. Tokura

Phys. Rev. B 51 (1995) 16491

[96] A. M. Ahmed, M. Boshta, R. Braunstein, V. Morchshakov, K. Bärner, C. P. Yang, J. R. Sun, G. H. Rao J. of Alloys and Compounds, 348 (2003) 23

[97] K. Bärner, M. Annaorasov, C. P. Yang, V. Morchshakov

Electrical field induced order-order transitions in rare earth manganites.

Phys. Stat. Sol. (b) 241, No. 5, 1088-1095 (2004)

[98] H. J. Krokoszinski and K. Bärner

J. Magn. Mater. 21, 221 (1980)

[99] A. J. Millis, P. B. Littlewood, B. I. Shraiman

Double Exchange Alone Does Not Explain the Resistivity of $\mathrm{La}_{1-\mathrm{x}} \mathrm{Sr}_{\mathrm{x}} \mathrm{MnO}_{3}$

Phys. Rev. Lett. 74, 5144 (1995)

[100] A. Chattopadhyay, A. J. Millis, D. Das Sarma

Optical spectral weights and the ferromagnetic transition temperature of colossal-magnetoresistance manganites: Relevance of double exchange to real materials

Phys. Rev. B 61, 10738 (2000)

[101] A. M. Ahmed, A. Kattwinkel, K. Bärner, C.P. Yang, J. R. Sun, G. H. Rao, P. Terzieff, H. Schicketanz Physica B 324, 102 (2002)

[102] Li Pi, Jianwei Cai, Quan Zhang, Shun Tan, Yuheng Zhang

Phys. Rev. B 71 (2005) 134418

[103] K. Bärner, K. Heinemann, I. V. Medvedeva

Phys. Stat. Sol. (b) 185, 455 (1994)

[104] J. C. Slonczewski

J. de Phys. C8 (1988) 1629

[105] H. Y. Hwang. S. W. Cheong, N. P. Ong, B. Batlogg

Spin-Polarized Intergrain Tunneling in $\mathrm{La}_{2 / 3} \mathrm{Sr}_{1 / 3} \mathrm{MnO}_{3}$

Phys. Rev. Lett. 77 (1996) 2041

[106] Charls Kittel

Einführung in die Festkörperphysik

R. Oldenbourg Verlag, München, Wien 1968

[107] T. Doi, K. Kohn

Phase Transitions 38 (1992) 273

[108] H. D. Sharma, Pawan Kumar, Abhishek Pathak and Ratnamala Chatterjee

Effect of rare earth ions on the electrical conductivity of modified PLnZT ceramics (5/65/35) $\mathrm{Ln}=\mathrm{Pr}^{+3}$, $\mathrm{Nd}^{+3}, \mathrm{Eu}^{+3}, \mathrm{Gd}^{+3}$ and $\mathrm{Dy}^{+3}$

Submitted to Phys. Stat. Sol. (2006)

[109] B. Jaffe, W. R. Cook and H. Jaffe

"Piezoelectric ceramics"

Academic Press, London and new York (1971) 
[110] M. E. Lines, and A. M. Glass

Principles and applications of ferroelectric end related materials.

Clarendon Press, Oxford (1979)

[111] Duane Dimos, W. Robert, Schwartz and Steven J. Lockwood J. Am. Cerem. Soc. 77, 11 (1994) 3000-3005

[112] R. von Helmolt, J. Wecker, B. Holzapfel, L. Schultz, and K. Samwer Giant negative magnetoresistance in perovskitelike $\mathrm{La}_{2 / 3} \mathrm{Ba}_{1 / 3} \mathrm{MnO}_{\mathrm{x}}$ ferromagnetic films Phys. Rev. Lett. 71, 2331 (1993)

[113] A. Urushibara et al. Insulator-metal transition and giant magnetoresistance in $\mathrm{La}_{1-\mathrm{x}} \mathrm{Sr}_{\mathrm{x}} \mathrm{MnO}_{3}$ Phys. Rev. B 51, 14103 (1995)

[114] G. H. Jonker and J. H. van Santen Physica ('s-Grav.) 16 (1950) 337-349

[115] J. H. van Santen and G. H. Jonker Physica ('s-Grav.) 16 (1950) 599-600

[116] J. Volger

Further experimental investigations on some ferromagnetic oxidic compounds of manganese with perovskite strukture.

Physica XX (1954) 49-66

[117] V. Morchshakov, M. Boshta, K. Bärner, I. Medvedeva, N. Guiblin, C. Martin and B. Raveau Evidence of photogenerated spase charges in the charge ordered state of $\operatorname{Pr}_{0.63} \mathrm{Ca}_{0.37} \mathrm{MnO}_{3}$ single crystals. Physica B 351 (2004) 171 



\section{Lebenslauf}

22.04.1977 geboren in Wedenka bei Kustanai (Kasachstan) als Sohn von Wladimir Morsakov und Lidia Bogdanova.

Staatsangehörigkeit: deutsch und kasachisch.

Sep. 1984 - Jun. 1994 Allgemeine Oberschule in Borowskoi bei Kustanai (10 Klassen).

Sep. 1994 - Jun. 1998 Physikstudium an der Universität in Kustanai.

Jun. 1998 Diplomprüfung

Sep. 1998 - Jun. 2000 Magisterstudium an der Universität-Kustanai.

Jun. 2000 Magisterprüfung („Halbleiterphysik und Mikroelektronik“).

Sep. 1998 - Jul. 1999 Physiklehrer am pädagogischen Kolleg in Kustanai.

Jul. 1999 - Jul. 2001 Programmierlehrer am bautechnischen Kolleg in Kustanai.

März. 2002 Beginn der Promotionsarbeit im 4. Physikalischen Institut der Universität Göttingen unter der wissenschaftlichen Leitung von Herrn Prof. Dr. K. Bärner

Aug. 2001 - Sep. 2004 Stipendiat des deutschen akademischen Austauschdienstes (DAAD)

Oktober 2004 Stipendiat der Otto-Vahlbruch-Stiftung 